CHANGING MOBILITIES

\title{
Mobility and Locative Media
}

Mobile communication in hybrid

spaces

Adriana de Souza e Silva and Mimi Sheller

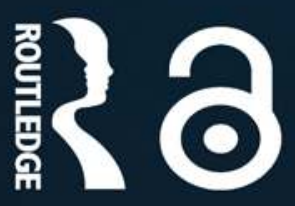




\section{Mobility and Locative Media}

Mobilities has become an important framework to understand and analyze contemporary social, spatial, economic, and political practices. Especially as mobile media become seamlessly integrated into transportation networks, navigating urban spaces, and connecting with social networks while on the move, researchers need new approaches and methods to bring together mobilities with mobile communication and locative media. Mobile communication scholars have focused on cell phones, often ignoring broader connections to urban spaces, geography, and locational media. As a result, they emphasized virtual mobility and personalized communication as a way of disconnecting from place, location, and publics.

The growing pervasiveness of location-aware technology urges us to rethink the intersection among location, mobile technologies, and mobility. Few studies have addressed the many transformations taking place in mobile sociality and in urban spatial processes through the appropriation of these technologies. This edited collection will address this gap by exploring the intersection of mobility, mobile communication, and locative media, as well as the implications of this for adjacent fields such as mobile art, mobile gaming, architecture, design, and urban planning.

Adriana de Souza e Silva is Associate Professor at the Department of Communication at North Carolina State University (NCSU), and Director of the Communication, Rhetoric and Digital Media (CRDM) program at NCSU.

Mimi Sheller is Professor of Sociology and directs the Center for Mobilities Research \& Policy at Drexel University. 
Changing mobilities

Series Editors: Monika Büscher, Peter Adey

This series explores the transformations of society, politics, and everyday experiences wrought by changing mobilities, and the power of mobilities research to inform constructive responses to these transformations. As a new mobile century is taking shape, international scholars explore motivations, experiences, insecurities, implications, and limitations of mobile living, and opportunities and challenges for design in the broadest sense, from policy to urban planning, new media and technology design. With world citizens expected to travel 105 billion kilometres per year in 2050, it is critical to make mobilities research and design inform each other.

\section{Elite Mobilities}

Edited by Thomas Birtchnell and Javier Caletrío

\section{Family Mobility}

Reconciling career opportunities and educational strategy

Edited by Catherine Doherty, Wendy Patton and Paul Shield

Mobility and Locative Media

Mobile communication in hybrid spaces

Adriana de Souza e Silva and Mimi Sheller

Forthcoming:

\section{Changing Mobilities}

Monika Büscher

\section{Cargomobilities}

Moving materials in a global age

Edited by Thomas Birtchnell, Satya Savitzky and John Urry

\section{Italian Mobilities}

Edited by Ruth Ben-Ghiat and Stephanie Malia Hom 


\section{Mobility and Locative Media}

Mobile communication in hybrid spaces

Adriana de Souza e Silva and Mimi Sheller 
First published 2015

by Routledge

2 Park Square, Milton Park, Abingdon, Oxon OXI4 4RN

and by Routledge

7II Third Avenue, New York, NY 10017

Routledge is an imprint of the Taylor \& Francis Group, an informa business

(c) 2015 Adriana de Souza e Silva and Mimi Sheller

The right of the editors to be identified as the authors of the editorial material, and of the authors for their individual chapters, has been asserted in accordance with sections 77 and 78 of the Copyright, Designs and Patents Act 1988.

All rights reserved. No part of this book may be reprinted or reproduced or utilized in any form or by any electronic,

mechanical, or other means, now known or hereafter invented, including photocopying and recording, or in any information storage or retrieval system, without permission in writing from the publishers.

Chapter 12 of this book is available for free in PDF format as Open Access at www.tandfebooks.com. It has been made available under a Creative Commons Attribution-Non Commercial-No Derivatives 3.0 license.

Trademark notice: Product or corporate names may be trademarks or registered trademarks, and are used only for identification and explanation without intent to infringe.

British Library Cataloguing-in-Publication Data

A catalogue record for this book is available from the British Library

Library of Congress Cataloging-in-Publication Data

Mobility and locative media : mobile communication in hybrid spaces / edited by Adriana de Souza e Silva, Mimi Sheller. -

I Edition.

pages $\mathrm{cm}-$ (Changing mobilities)

I. Communication-Social aspects. 2. Mass media-Social aspects. I. Silva, Adriana de Souza e, editor of compilation. II. Sheller, Mimi, editor of compilation.

HMI 206.M6266 20I4

302.230973-dc23

ISBN: 978-I-I38-778। 3-9 (hbk)

ISBN: 978-I-3। 5-77222-6 (ebk)

Typeset in Times New Roman

by Wearset Ltd, Boldon, Tyne and Wear 
To Matteo (Adriana)

To Eve, Ally and Dan (Mimi) 
This page intentionally left blank 


\section{Contents}

List of figures $\quad \mathrm{x}$

List of tables xii

Notes on contributors xiii

Acknowledgments $\quad$ xix

Introduction: moving toward adjacent possibles 1

ADRIANA DE SOUZA E SILVA AND MIMI SHELLER

\section{PART I}

Rethinking cohesion, coordination, and navigation

1 Mobile phones and digital Gemeinschaft: social cohesion in the era of cars, clocks and cell phones

RICH LING

2 Walking in the hybrid city: from micro-coordination to chance orchestration

ROBIN VAN DEN AKKER

3 Direct video observation of the uses of smartphones on the move: reconceptualizing mobile multi-activity

CHRISTIAN LICOPPE AND JULIEN FIGEAC

4 Rerouting borders: politics of mobility and the Transborder Immigrant Tool 


\section{PART II}

Performing location, place-making, and mobile gaming

5 Online place attachment: exploring technological ties to physical places

RAZ SCHW ARTZ

6 Location as a sense of place: everyday life, mobile, and spatial practices in urban spaces

DIDEM ÖZKUL

7 Performing city transit

TAIEN NG-CHAN

8 Location-based gaming apps and the commercialization of locative media

DALE LEORKE

9 Houses in motion: an overview of gamification in the context of mobile interfaces

NATHAN HULSEY

\section{PART III}

Mobile cities: mapping, architecture, and planning

10 Exploring locative media for cultural mapping

PETER HEMMERSAM, JONNY ASPEN, ANDREW MORRISON, IDUNN SEM, AND MARTIN HAVNØR

11 Designing for mobile activities: WiFi hotspots, users, and the relational programming of place

MICHAEL R. DOYLE

12 The power of place and perspective: sensory media and situated simulations in urban design 
13 The will to connection: a research agenda for the "programmable city" and an ICT "toolbox" for urban planning

OLE B. JENSEN

\section{Epilogue}

14 Restless: locative media as generative displacement TERI RUEB

Index 


\section{Figures}

3.1 A simple portable set-up to record the audio-video flux on Android-based smartphones

3.2 and 3.3 In all images the screen capture appears on the left, and the camera glasses recording on the right (both have been synchronized). In both images the user is sitting and using the smartphone in his lap, which is partly (3.2) or not at all (3.3) visible

3.4 Arriving at a red light behind the stopping traffic

3.5 Taking such an occurrence as an opportunity to gaze down, put the phone on the steering wheel and launch the Facebook application

3.6 A typical visual display for the circular progress bar

3.7 Sending a message and getting the circular progress bar 56

3.8 The right hand goes to the right to engage a gear 56

3.9 The gaze moves up to look at the road and "discover" that the light is still red

3.10 She immediately gazes down at the smartphone

3.11 The driver is scanning down her list of Facebook posts. The sudden motion of surrounding cars is detectable in data through the side window though not visible in the picture

3.12 She eventually looks up, and a large opening is now visible before her car, materializing the delayed character of her response

3.13 The black car on the right "jumps" into the gap, before she eventually starts to move her car forward again

4.1 The Transborder Immigrant Tool interface at work 70

5.1 Check-in screens $\quad 87$

5.2 Mayorship change message 93

10.1 Mapping results were displayed real-time in a local shop window

10.2 The map changed interactively as mapping was conducted 178

$\begin{array}{ll}10.3 & \text { Example of sample point notation by students } \\ \end{array}$ 
10.4 Use of the app led students to see the city in a new way

11.1 Distribution of ZAP Québec WiFi hotspots on the Québec City metropolitan area by type

11.2 Distribution of the most frequented ZAP hotspots

11.3 Top 25 percent most frequented hotspots and public transport

11.4 Pub Galway offers pub-goers a view of the activity on Avenue Cartier

11.5 Graphic representation of the WiFi user (A) with views of indoor (B) or outdoor (C) activities

12.1 The Roman Forum sitsim showing a now-and-then snapshot of the Via Sacra looking toward the Temple of Caesar

12.2 Example of perspective combining high altitude bird's-eye overview and distance

12.3 Example of perspective combining street level, close up and detail. Perspectives combining large frame, wide angle, and distance are absent from the presentations

12.4 The double perspective as viewed from the starting point at a distance of about 75 meters and facing the old building of the museum

12.5 Moving toward the left (northern) side of the old building more details of the planned museum appear

12.6 Old and new. The stone wall of the new building points in the direction of the main entrance in the atrium behind the old building

12.7 Using the fly-in function the user can tilt and pan the device to look around in the simulated Munch room

14.1 Teri Rueb, Drift, 2004, Walker on the tidal flats

14.2 Teri Rueb, Core Sample, 2007, Visitors in the gallery and walkers on the island

14.3 Teri Rueb, Elsewhere: Anderswo, 2009, Walker in the botanical garden

14.4 Teri Rueb and Larry Phan with contributions from Carmelita Topaha (Diné), No Places With Names, 2012, Walker holding sculpture 


\section{Tables}

11.1 Characteristics of the three WiFi user groups

193

11.2 ZAP Québec WiFi hotspots by type and status of inclusion in study as of September 2010

11.3 Hotspots visited as part of the spatial analysis 


\section{Contributors}

Jonny Aspen is Associate Professor at the Institute of Urbanism and Landscape, Oslo School of Architecture and Design. His research focuses on urban planning history, city cultures, urban theory, contemporary urban development, and digital media and cities. He has edited a book on urban studies in Norway concerning contemporary issues of gentrification and immigration, and a Norwegian anthology on international urban theory. His PhD was on issues of city planning and urban representation. He is a senior researcher in a project on social media, design and the city, YOUrban, at the Oslo School of Architecture and Design. He is currently, with John Pløger, writing a book on vitalism and urbanism.

Adriana de Souza e Silva is Associate Professor of Communication at North Carolina State University (NCSU), affiliated faculty at the Digital Games Research Center, and Director of the Communication, Rhetoric and Digital Media (CRDM) program at NCSU. Her research focuses on how mobile and locative interfaces shape people's interactions with public spaces and create new forms of sociability. She teaches classes on mobile technologies, location-based games, and internet studies. She is the co-editor (with Daniel M. Sutko) of Digital Cityscapes-Merging Digital and Urban Playspaces (Peter Lang, 2009), the co-author (with Eric Gordon) of Net Locality: Why Location Matters in a Networked World (Blackwell, 2011), and the co-author (with Jordan Frith) of Mobile Interfaces in Public Spaces: Control, Privacy, and Urban Sociability (Routledge, 2012). She holds a PhD in Communication and Culture from the Federal University of Rio de Janeiro, Brazil.

Michael R. Doyle holds a Master in Architecture and a Master of Science from Laval University (Québec, Canada) and a Bachelor of Science in Architecture from the University of Cincinnati in Ohio. He has worked for architecture firms in Chicago (US), Cleveland (US), and Paris (France). Before beginning his doctoral research at the École Polytechnique Fédérale de Lausanne (EPFL) in Switzerland in 2012, he worked for the Interdisciplinary Research Group on the Suburbs (GIRBa) at the Laval University School of Architecture where he helped to develop a metropolitan-level internet survey investigating 
housing, mobility, and ICT use. His Master's research project addressed WiFi use and the spatial configurations of WiFi hotspots in Québec City. As a PhD researcher for the Deep City project at the EPFL, he is now working on urban underground spaces.

Fernanda Duarte is a $\mathrm{PhD}$ candidate at the Communication, Rhetoric, and Digital Media (CRDM) program at North Carolina State University (NCSU) with funding from the Ministry of Education in Brazil and the Fulbright Program at the US Department of State. Her research interests are mobilities, media technologies, politics, and digital poetics. Her current research focuses on applications of pervasive computing and the rising issues of intimacy and privacy in biopolitics and activism/art.

Julien Figeac is a CNRS researcher at the Information Processing and Communication Laboratory, Telecom ParisTech, France. His research and publications focus on the uses of smartphones in situations of mobility. He contributes to the development of a sociological method based on video recordings, video-ethnography, to analyze how information and communication technologies (ICTs) are changing the organization of social interactions and urban mobility. He has developed this method in different case studies: the uses of mobile TV in public transportation in Paris, the modalities of participation in mobile social networking, and the forms of sociability generated by geosocial networking and location-based games.

Martin Havnør holds a degree in computer science from the University of Oslo and is currently working as CTO at Faster Imaging AS. With a background from the Scandinavian demo-scene, he has a passion for software development with tight requirements such as memory footprint and available processing power. Through his position at Faster Imaging he has also gained a keen interest in maps and everything GIS-related, and held talks on related subjects at events such as AppWorks and State Of The Map. An interest in digital art has also led to the participation in installations and projects related to interactivity, mobility, and performativity.

Peter Hemmersam is Associate Professor at the Institute of Urbanism and Landscape at the Oslo School of Architecture and Design. His PhD focused on urban design, public urban space, and retail, using a variety of architectural mapping methods. Recently, his research has focused on sustainable urban design, ecological urbanism, and eco-cities in a Scandinavian perspective. In addition he researches changing circumpolar landscapes, employing architectural methods of perceptual mapping of material landscapes. He has previously been a practicing architect with a focus on urban design, and worked as an educator and researcher at the Aarhus School of Architecture in Denmark. He has worked on several projects for CHORA and Raoul Bunschoten using experimental mapping methods. From 2003 to 2007 he was the Head of the Institute of Urbanism AHO. 
Nathan Hulsey is a doctoral candidate at the Communication, Rhetoric and Digital Media (CRDM) program at North Carolina State University. His current research involves a critical approach to the history of gamification, focusing on surveillance practices, spatiality, and biopolitics. He also contributes research to the fields of game studies and mobilities.

Ole B. Jensen is Professor of Urban Theory at the Department of Architecture, Design, and Media Technology, Aalborg University (Denmark). He holds a BA in Political Science, an MA in Sociology, and a PhD in Urban Planning. $\mathrm{He}$ is co-founder and board member at the Centre for Mobilities and Urban Studies (C-MUS), Aalborg, and Task Force Member in the Cosmobilities Network. His main research interests are within Urban Mobilities and Urban Networked Technologies. He is the co-author of Making European Space: Mobility, Power and Territorial Identity (Routledge 2004, with Tim Richardson) and author of Staging Mobilities (Routledge, 2013) and Designing Mobilities (Aalborg University Press, 2014).

Dale Leorke is a $\mathrm{PhD}$ candidate in the School of Culture and Communication at the University of Melbourne, Australia. His thesis examines location-based gaming and play in public space, drawing on case studies of games and artistic projects that use mobile and location-aware devices to bring digital play into urban space. His most recent work can be found on his research page: http://unimelb.academia.edu/DaleLeorke.

Christian Licoppe is a Professor of Sociology at the Department of Social Science in Telecom Paristech in Paris. Trained in history and sociology of science and technology, he has worked for a stretch in industrial research, where he managed social science research at Orange R\&D, before taking his current academic position. Among other things he has worked in the field of mobility and communication studies for several years. He has used mobile geolocation and communication data to analyze mobility and sociability patterns of mobile phone users. He has studied various phenomena related to the proliferation of mediated communication events and "connected presence." He has also studied extensively the uses of location-aware games and proximity-aware mobile technologies communities. His recent work in mobile communication has focused on the development of methods to record and analyze the use of mobile communication in "natural" situations (such as mobility and transport settings) and on the use of ethnomethodology and conversation analysis to understand the organization of mobile communication as in Skype and mobile video calls.

Gunnar Liestøl is professor at the Department of Media and Communication, University of Oslo. He has authored and edited numerous books and articles on rhetoric, narrativity, and digital design, among them Digital Media Revisited (MIT Press 2004). He has a two-decade-long track record of digital design for various platforms, starting with early hypermedia systems for 
museums, CD and the Web, such as the award-winning Interactive Kon-Tiki (1995). He focuses on critical construction as a combination of practical and theoretical experiments based in and informed by humanistic disciplines and competencies. For the past several years he has been exploring the fields of mobility, locative media, and indirect augmented reality for simulation of past and future environments in situ. Several of these simulations have been published as mobile apps, such as the Roman Forum, and are available for free download at the Apple App Store and Google Play.

Rich Ling ( $\mathrm{PhD}$, University of Colorado in Sociology) is the Shaw Foundation Professor of Media Technology at Nanyang Technological University in Singapore, works at Telenor Research and has an adjunct position at the University of Michigan. Ling has studied the social consequences of mobile communication for the past two decades. From 2008 to 2014 he was a professor at the IT University of Copenhagen. He has written several books including The Mobile Connection (Morgan Kaufmann 2004), New Tech, New Ties (MIT 2008) and most recently Taken for Grantedness (MIT 2012). He is a founding co-editor of the journal Mobile Media and Communication (Sage) and the Oxford University Press series Studies in Mobile Communication.

Andrew Morrison is Professor of Interdisciplinary Design at the Institute of Design (IDE), Oslo School of Architecture and Design where he is Director of the Centre for Design Research (www.designresearch.no). He takes part in and leads a range of design research projects. These cover Communication and Interaction Design, dynamic interfaces and social media, and locative media; RFID, emerging technologies, mediation, and activity; Service Design and innovation; electronic arts installation; narrative and mobile media; practice-based research/research by design; online research mediation; and doctoral education in design and design research methods. Andrew also focuses on design writing, fiction, and criticism. He has published widely in design, new media, and electronic arts. He has coordinated the crossdisciplinary doctoral school at AHO and is on the board of several international journals, the Swedish National PhD Design School, and the Board of AHO. He has a BA in Law and English, an MA in English, an MSc in Applied Linguistics and a $\mathrm{PhD}$ in digital media. Prominent current projects include YOUrban on social media and the city, and Future North on prospective views on the arctic in design and landscape architecture. His recent books are Inside Multimodal Composition (2010) and Exploring Digital Design (2010).

Taien Ng-Chan is a writer, artist, and scholar in the $\mathrm{PhD}$ in Humanities at Concordia University in Montréal, Canada. Her work investigates everyday urban life through experimental cinema, cartography, poetry, and documentary, in interdisciplinary, multimedia contexts. She has published two books of poems, edited two anthologies, and produced a multimedia $\mathrm{CD}$; as well, she 
has written drama for stage, screen, and radio. Her video and media works have been shown across Canada, in the US, Korea, Sweden, and the UK. She is a founding member of the Hamilton Perambulatory Unit (HPU).

Didem Özkul (PhD, Communication and Media Research Institute, University of Westminster) is a research fellow, working for the UK's Arts and Humanities Research Council-funded research project, Digital Engagements, based at CAMRI, University of Westminster. Her PhD research concerns people's use of mobile communication technologies in urban spaces, particularly focusing on spatial perception and locational information use. Employing creative visual methodologies in order to explore and visualize transformations in the perception of urban space, her research offers an analysis of the relationships between the use of place-specific information on mobile communication technologies and different aspects of place-making and self-presentation in urban spaces. Details about her research can be found at www.mobilenodes.org

Teri Rueb works at the intersection of interactive media, sound, land, and environmental art. She pioneered the form of GPS-based interactive installation with her project "Trace" which was developed at the Banff Centre for the Arts in 1996. She is currently an Artist Resident at the metaLab at Harvard University. She is the recipient of numerous awards including a Prix Ars Electronica Award of Distinction in 2008 for her project "Core Sample" set on a landfill in the Boston Harbor. Her work has been presented internationally and funded by the Arnold Arboretum/Harvard, the Banff Center for the Arts, Edith Russ Site for Media Art, Klangpol, LEF Foundation, Turbulence. org, Artslink, and La Panacée Centre Pour Culture Contemporaine, among others. She is currently Professor of Media Study at the State University of New York at Buffalo (SUNY) where she also holds an affiliate appointment in the Department of Architecture and Planning. She is Founder and Director of Open Air Institute, a platform for connecting field-based learning and collaborative partnerships at the intersection of media, ecology, and culture. She holds a doctorate from Harvard University where her research focused on landscape, subjectivity, and mobile network society.

Raz Schwartz is a postdoctoral researcher at Cornell Tech NYC and a Magic grant fellow at the Brown Institute for Media Innovation at Columbia Journalism School. He studies social media usage in urban settings and focuses on examining local social interactions by applying computational social science methods. Prior to joining Cornell Tech NYC, he was a postdoctoral researcher at the Social Media Information Lab at Rutgers University. He completed his $\mathrm{PhD}$ in the STS program at Bar-Ilan University and was a visiting scholar in the Human-Computer Interaction Institute at Carnegie Mellon University. He holds a Master's degree in Law from Bar-Ilan University and a Bachelor's degree in Communications and Economics from Tel-Aviv University. His research on location-based social networks was presented in 
various academic settings and was featured in media outlets such as the Wall Street Journal, Wired, Rhizome, and The Atlantic.

Idunn Sem is Head Engineer/Media Developer at EngageLab, University of Oslo, and has a degree in Literature and a cant. philol. degree in Media Studies from the University of Oslo. She takes on the mediator role between researchers and developers at EngageLab and has a varied portfolio of projects and co-written publications. Her research looks into practice-based inquiries of digital media, exhibition design, electronic arts, embodied interaction, choreography and digital scenography, and electronic mediation of research (research rhetoric).

Mimi Sheller is Professor of Sociology and founding Director of the Center for Mobilities Research and Policy at Drexel University in Philadelphia. She is founding co-editor of the journal Mobilities; Associate Editor of Transfers: Interdisciplinary Journal of Mobility Studies, and co-editor of The Routledge Handbook of Mobilities (2013). As co-editor, with John Urry, of Mobile Technologies of the City (2006), Tourism Mobilities: Place to Play, Places in Play (2004), and co-author of the article "The new mobilities paradigm" in their special issue of Environment and Planning A (2006) on Materialities and Mobilities, she helped to establish the new interdisciplinary field of mobilities research. She has also authored several books in Caribbean Studies, including Democracy After Slavery (2000), Consuming the Caribbean (2003), Citizenship from Below (2012), and Aluminum Dreams (2014).

Robin van den Akker is a cultural philosopher working as a doctoral researcher at the Faculty of Philosophy of Erasmus University Rotterdam and as a lecturer at Erasmus University College. He is co-founding editor of the popular academic webzine "Notes on Metamodernism" and co-coordinator of the "Centre for Art and Philosophy." Robin teaches, writes, and speaks on contemporary aesthetics and culture, the digitization of everyday life, and social space and social time. He acted as advisor for various art exhibitions and cultural events. 


\section{Acknowledgments}

This volume is indebted to the energy and collaborative spirit of the PanAmerican Mobilities Network, which was founded in 2010 by a group of scholars working in the field of mobilities research, but also in conversation with scholarship in media and communication, anthropology, cultural geography, sociology, and other fields concerned with movement, spatiality, infrastructure, mobile technology, and the politics of mobility. The first conference of the network was organized by Phillip Vannini and hosted at Royal Roads University on Victoria Island, BC, and we first want to especially thank Phillip for instigating all of us to come together.

The co-editors of this volume then went on to host the second and third conferences of the network, through which an exciting exchange of international scholarship has begun to form. Sheller hosted "Mobilities in Motion" at Drexel University in 2011 at which de Souza e Silva gave one of the keynote addresses and a number of communication scholars presented work, beginning the process that led to this book. We want to thank the organizing committee who, along with the two of us, helped to set in motion this ongoing multidisciplinary and transnational exchange: Malene Freudendal-Pedersen, Jennie Germann-Molz, Ole B. Jensen, Paola Jiron, Sven Kesselring, and Phillip Vannini. Many thanks also to the Dean of the College of Arts and Sciences Donna Murasko, Marion DiBattista and other staff members in the Dean's Office, the Cosmobilities Network Steering Group, and members of the Pan-American Mobilities Network for making this conference possible. Thanks also to other colleagues at Drexel who have supported my interest in mobile media, in particular Hana Iverson who became a key collaborator and friend in this field.

The following year de Souza e Silva hosted the network's third annual conference "Local and Mobile: Linking mobilities, mobile communication and locative media" in association with the $\mathrm{PhD}$ program in Communication, Rhetoric and Digital Media (CRDM) at North Carolina State University (NCSU) in 2012. The idea for this volume arose out of that conference and several of the contributors presented work there, including keynote speakers Rich Ling and Teri Rueb. We thank members of the organizing committee, Heather Horst, Lee Humphreys, Ole B. Jensen, Irina Shklovski, and Phillip Vannini, as well as our 
co-hosts in the CRDM program, and the Department of Communication at NCSU who helped make this conference possible. We would like to thank all the students who, with their hard work, made the conference possible, and particularly: Elizabeth Johnson-Young, for being Adriana's right hand in the conference organization; Fernanda Duarte for the website design and conference visual identity; Seth Mulliken for the audio and video recording and editing; and Dyvia Vijay-Pratheek for the photography.

Mimi Sheller would like to thank Dan Schimmel for introducing her to many aspects of internet culture and mobile technology, without which her work would have never turned in this direction. From internet dating where we first met, to MySpace which we explored together, and eventually a launch onto Facebook, Twitter, Tumblr, Instagram, Foursquare and more: thank you for being my mobile media companion, place-maker, and ever-connected analog/digital presence. And to my girls, Ally and Eve: put that iPad down! I'd rather get a hug.

Adriana de Souza e Silva would like to thank her son Matteo for being a constant presence while this book was taking shape. He attended the 2012 conference in my belly when I was six months pregnant, and patiently slept or nursed in my arms while I reviewed and edited the chapters for this collection. During the last round of revisions he was already running around, but still loved the computer. And soon he will be able to read this book! 


\title{
Introduction
}

\section{Moving toward adjacent possibles}

\author{
Adriana de Souza e Silva and Mimi Sheller
}

Steven Johnson (2010) in his book Where Good Ideas Come From introduces Stuart Kaufman's concept of the "adjacent possible." The adjacent possible can be understood as the untapped potential of a living organism. In evolutionary terms, the idea of the adjacent possible supported that while there were a myriad of possibilities for new forms of life to emerge throughout history, only specific forms of life could actually have appeared at certain moments in time. This is because their shape, features, and properties have always been contingent on preexisting life forms. For example, the appearance of human beings on Earth has been a possibility since the first carbon atoms started to combine into more complex single cell organisms. However, a human being could not have appeared a few million years ago, because it was not the adjacent possible of a carbon molecule. In between the carbon molecule and the human being, there were multiple necessary steps in the history of evolution; multiple adjacent possibles. As Johnson (2010: 31) puts it,

What the adjacent possible tells us is that at any moment the world is capable of extraordinary change, but only certain changes can happen. The strange and beautiful truth about the adjacent possible is that its boundaries grow as you explore those boundaries.

Although Kauffman developed his adjacent possible theory to address biological systems, Johnson applies it to the development of ideas and knowledge. He suggests that good ideas are often generated by the recombination of other existing ideas. According to Johnson, "good ideas are not conjured out of thin air; they are built out of a collection of existing parts, the composition of which expands (and, occasionally, contracts) over time" (2010: 35).

Mobility and Locative Media: Mobile Communication in Hybrid Spaces emerged from the desire to explore the adjacent possibles that emerge when scholars from three disciplinary areas - mobile communication, mobility, and locative media-come together to explore and challenge these boundaries. In editing this volume, we hoped not only to explore each single area, but to investigate what new ideas and knowledge could emerge when we push and break 
those boundaries. Indeed, in exploring the rich potentials of those edges we are also attuning our research to the adjacent possibles that are already emerging through the experimentation of artists, early adopters, innovators and everyday users who themselves are exploring the boundaries of emergent mobile technologies through their own spatial practices. Mobile communication, mobility, and locative media grew as independent research areas out of the recognition that new practices around mobility, mobile technologies, and location-awareness are becoming key elements to understand social, spatial, and cultural relationships in today's world. Each of these areas have also emerged as an interdisciplinary effort to explore the adjacent possibles in their own disciplines.

The Pan-American Mobilities Network (founded in 2010) and the Cosmobilities Network (founded in 2004) are excellent examples of scholars from different disciplines coming together to explore new ideas related to mobilities. Involving researchers from sociology, anthropology, communication, urban studies, and geography, just to name a few, mobilities scholars focus on the systematic movement of people, goods and information that "travel" around the world, the complex infrastructures that enable (or prevent) such movements, as well as the practices, habits and affective experiences that are associated with different potentials for movement, stillness, pausing and waiting (Sheller and Urry 2006a; Hannam et al. 2006). As such, the emerging interdisciplinary field of mobilities research challenges traditional scholarship that often ignores the social, cultural, and experiential dimensions of co-constituted immobilities and mobilities (Kellerman 2006; Jensen 2009). Travel, movement, communication and transportation networks help to constitute modern bodies, societies, cities, regions, and borders. These dynamically interpenetrating choreographies, scales, and rhythms of mobilities (and immobilities) (Edensor 2011) also (re)configure spatiotemporalities, performatively shaping spatial relations into what some theorize as "movement-space" (Thrift 2008).

Mobilities has become an important framework to understand and analyze contemporary social, spatial, economic and political practices (Adey et al. 2014). The orchestrated movement of people, goods and information that "travel" and "traffic" the streets, rails, air space, oceans, fiber-optic cables, cell towers, servers, and satellites of the world depend on the infrastructures, "moorings" and mobility regimes that channel and regulate entry and exit, speed and wait-times, mobilizations and demobilizations. Mobility has always been critical for the creation of social networks (or what Elliott and Urry [2010] call "network capital") and to the development of connections to places and potentials for movement (understood as "motility" [Kaufman et al. 2004]). Thus mobility is always imbued with normative questions of unequal access, rights and capabilities. In addition, mobilities research contributes new approaches to the technological, social and cultural developments in transportation, border control, mobile communication, "intelligent" infrastructure, and surveillance. In particular it focuses attention on how these networked infrastructures are extended and appropriated by agents of both governance and resistance (e.g., Graham and Marvin 2001; Packer 2008; Vukov and Sheller 2013). 
While mobilities scholars also focus on communication networks, there is another set of scholars whose specific focus on cell phones emerged from the recognition that communication media has become mobile. Coming mostly from media and communication studies, these scholars started inquiring what happens to social relations when we can communicate while on the move (Ling 2004; Katz and Aakhus 2002; Brown et al. 2002). More specifically, they focused on the ways mobile communication technologies (that is, cell phones) change the way we communicate, interact with space, and with others who are both nearby and remote (Campbell 2013; Goggin and Wilken 2012). Mobile phones had received very sparse scholarly attention until the end of the last century, but in the first decade of the twenty-first century they have become one of the most popular communication media today, surpassing the number of TV sets and landline phones (Rice and Katz 2003). The study of mobile communication is also interdisciplinary, spanning the fields of sociology, anthropology, communication, economics, and computer science, just to name a few. Although both mobility and mobile communication studies gained recognition around the same time, they lived parallel lives. Scholars in each of these fields had their own set of interests, methods, conferences, and publication venues.

Mobile communication scholars have traditionally focused on cell phones as interpersonal communication devices, often ignoring broader connections to urban spaces and geography, which has been a central focus of mobilities studies. Furthermore, both mobile communication scholars and mobility scholars largely focused on the use of cell phones as two-way communication devices, used for voice and text messages. There is a vast literature on the use of mobile phones for micro-coordination (Ling and Yttri 2002, 1999), formation of social networks via voice calls and SMS (Keyani and Farham 2005; Harper et al. 2005; Habuchi 2005), and virtual mobility (Kellerman 2006; Urry 2003; Larsen et al. 2006). As a result, they often emphasized virtual mobility or the privatization of public space as ways of disconnecting from place and location (Gergen 2002; Puro 2002), in contrast to mobility theorists who were beginning to understand the mobile transformations and blurred boundaries of public and private (Sheller and Urry 2003). And, for a long time, there was one element missing from the study of mobility and mobile communication: location.

Locative media artists were the very first ones to foresee the possibilities of using mobile technologies (including GPS devices and cell phones) to reframe the relationships between people and spaces (Hemment 2004, 2006; Tuters and Varnelis 2006; Rueb 2007). When most people were still thinking about mobile phones as mobile landlines, locative media artists were already trying to figure out what happens when people used their phones not only to talk to remote people, but also to engage with digitally located information and the physical space around them (Rueb 1999; Blast-Theory and The-Mixed-Reality-Lab 2001; Paulos and Goodman 2003). Many of their projects, developed within the artistic community and inside research labs, were unknown to the general public until very recently, when the popularity of smartphones such as the iPhone and the 
phones that use the Android system made location-awareness a common feature of our experience of mobility through space (de Souza e Silva and Frith 2010; Gordon and de Souza e Silva 2011). Mobile locative artists continue to remain at the forefront of experimentation with the possibilities emerging from the interaction between embodied and sensory practices, locational awareness and positioning, and the annotation and augmentation of urban and non-urban spaces (Iverson et al. 2014).

Location-based services, such as augmented reality browsers and Yelp, location-based social networks, such as Foursquare and Latitude, and GPS devices, all highlight the increasing importance of location as a mediator of our social and networked interactions. As a consequence, we are experiencing a shift in the way we interact with both the internet and physical spaces, in which location becomes the organizational logic and even, in ways, the currency of networked interactions (Gordon and de Souza e Silva 2011). Today, increasingly, the kinds of information (people, places, images) we access online depends on our physical location. We experience this phenomenon with trivial activities. For example, every time someone opens Google Maps on a smartphone, the map centers around the user, and all their searches are location-specific. Every time someone performs a Google search on a computer, they receive results depending on their location, which is determined by their computer's IP address. A search result in New York will be different than a search result in Los Angeles, which will be different from a search result in São Paulo.

Our position, defined by latitude and longitude coordinates, becomes our entrance to the internet. According to this logic, location works as a filter that determines the types of information we access and the way we interact with the spaces around us. The pervasiveness of location-aware technology has made it possible to locate ourselves and be networked while on the move. As usergenerated maps and location-aware mobile devices become commonplace, we experience a shift in the way we connect to other people and to information as we move through space. And such locational awareness is increasingly being seamlessly integrated into transportation networks, including cars, airports, transit networks, and a range of new applications for accessing and sharing vehicles, determining walkability, or navigating between a wide range of location-based transport options (Sheller and Urry 2006b). Networked interactions permeate our world, our social lives, our work practices, and our political practices.

In the first instance, we no longer enter the internet-we carry it with us. We access and appropriate its diverse affordances while moving through physical spaces. Mobile phones, GPS receivers, and RFID tags are only a few examples of location-aware mobile technologies that mediate our interaction with networked spaces and influence how we move in these spaces. Second, our physical location determines the types of information with which we interact, the way we move through physical spaces, and the people and things we find around us. These new kinds of networked interactions manifest in everyday social practices 
that are supported by the use of mobile and location-aware technologies, such as participation in location-based mobile games and social networks, use of location-based services, development of mobile annotation projects, and social mapping, just to name a few. Third, the engagement with these practices has important implications for identity construction, our sense of privacy, our notions of place and space, civic and political participation, policy making, as well as forms of work, cultural production and consumption in everyday life. Being able to consistently and persistently locate ourselves (or perhaps cloak our location) within this mobile digital network through location-aware technologies fundamentally changes how we understand both the internet and the physical space around us (Gordon and de Souza e Silva 2011).

Therefore, we also need different theoretical frameworks, methodologies and research questions to understand the social, spatial and political changes that happen today as they relate to the use of location-aware technologies in our complexly mobile worlds. Scholars in each of the fields we have described are exploring new methodologies for grasping the dynamic cultural landscapes and spatiotemporal performances at the intersection of mobility, mobile communication, and locative media. Mobilities researchers have been developing new "mobile methods" that seek to engage new research questions in ways that do justice to all the dimensions of mobility (Sheller and Urry 2006a; Büscher et al. 2010; Fincham et al. 2010). Many of these methods make use of mobile communication devices, GPS mapping, or locational interactions to try to record and capture movement, or "negotiation in motion" in new ways (Jensen 2010). Communication scholars are also experimenting with new ways of tracking, measuring, theorizing, and analyzing mobile communication, or new ways of using mobile locative media that allow them "to enter the field," so to speak (Campbell 2013; Pearce 2013; Goggin 2013).

This edited collection emerged from the desire to explore the adjacent possibles that might actualize when scholars from these three interrelated areas are in contact with each other, and to create a network among scholars who are doing very similar, but sometimes parallel works. Studying mobilities today means engaging with mobile communication technologies, and locative media. Likewise, mobile communication studies require an understanding of mobility practices. New transdisciplinary centers such as the Centre for Mobilities Research (Lancaster University), the Center for Mobilities Research and Policy (Drexel University) or the Centre for Mobility and Urban Studies (Aalborg University) are building multidisciplinary scholarship at the intersection of these fields as well as other adjacent areas such as urban studies, design, architecture, and planning, all of which come into play in this volume. Contextualizing studies within the framework of mobilities and mobile communication also requires studying cell phones as locative devices. It is ironic that transdisciplinarity has become such a key term to drive research methodologies and collaborations in academia today, and yet new "multidisciplinary areas," such as mobile communication, mobilities, and locative media, have developed independently from each other. 
Few studies have even begun to address the many transformations taking place in mobile sociality and in urban spatial processes through the appropriation of these new technologies of mobile and locational communication. More importantly, it is no longer possible today to develop scholarship within mobility studies without being in dialogue with mobile communication and locative media scholars, and this requires a better understanding of specific practices, technologies, and applications. Mobile communication and locative media scholars will also benefit from incorporating mobilities theoretical frameworks in their approaches to studying mobile technologies, including a stronger spatial dimension, renewed attention to the body, the gaze, and the haptic senses, and a greater attention to the wider social and political contexts of mobile media.

All of the research fields are also beginning to benefit from greater interaction with artists, architects, designers, and software developers who are also working with mobile and locational technologies. Through such interactions there are also beginning to emerge other adjacent possibles based on the cross-pollination of artist/theorists, maker/researchers, designer/lecturers, social entrepreneur/ teachers, and many other new hybrids. At the boundaries of our work these new possibilities open up myriad opportunities for innovation in thinking and doing, communicating and learning, sharing and translating across fields. We hope this book extends some of those possibilities.

\section{The book}

The chapters in this edited collection are at the intersection of mobility, mobile communication, and locative media. We call this a hybrid space, both in the sense of the hybridity of digital information and physical spatiality, and in the sense of a hybrid crossing of various disciplinary boundaries. We come to this project as a scholar of mobile locative communication (de Souza e Silva) and a scholar of the sociology of mobilities (Sheller) - and we ourselves crossed paths through participation in the Pan-American Mobilities Network. Sheller hosted their second annual conference on "Mobilities in Motion" at Drexel University in 2011, at which de Souza e Silva gave a keynote address and a number of communication scholars presented work; and then de Souza e Silva hosted their third annual conference "Local and Mobile: Linking mobilities, mobile communication and locative media" with the program in Communication, Rhetoric and Digital Media at North Carolina State University in 2012, at which several of the contributors to this volume presented work. We see this as the beginnings of a fruitful co-mingling of scholarship, theories, and methodological discussions across these fields, which has attracted students interested both in mobile technologies and in areas such as movement, urban spatiality, place-making, mobile art and the politics of mobility.

The volume is divided into three parts, each addressing key issues that emerge from the explorations of the adjacent possibles in these three disciplinary areas. The first, "Rethinking cohesion, coordination and navigation," focuses on new 
ways of thinking about issues of place, sociability and coordination while moving through space. Against earlier theorists such as Sherry Turkle (2011) or Robert Putnam (2000) who emphasized the social fragmentation and aloneness brought about by privatized technologies of electronic communication, each of the contributors in this part investigates the complex ways in which people are actually appropriating mobile technologies into everyday practices that enable connectivity or togetherness. Rather than a duality that would set off place, personal intimacy, and proximity against mobility, abstract systems, and distanciation, the research presented here finds people creatively incorporating mobile technologies into ongoing forms of sociality, domesticity, sharing, and conviviality that cross borders and blur boundaries of various kinds.

In Chapter 1, "Mobile phones and digital Gemeinschaft: Social cohesion in the era of cars, clocks and cell phones," Rich Ling lays the theoretical groundwork for the book by examining Ferdinand Tönnies' (1965) tension between Gemeinschaft and Gesellschaft through the lens of mobile phone use and its relation to other forms of mobility. Far from being a technology for regulation, control, and alienating systematization of modern society, he describes how mobile communication coincides with the rise of suburban cultures of automobility in ways that enable people to maintain their intimate social spheres - or what Tönnies would call Gemeinschaft. Ling also investigates the synergy between car-based transportation and mobile communication, by defining them both as "technologies of social mediation." As we shall read throughout this volume, mobile technologies are deeply performative of belonging, a sense of place, even poetics and aesthetics, despite the fact that tensions remain with more systems-based elements such as commercialization and surveillance.

While Ling discusses mobile communication in general, in the second chapter Robin van den Akker builds a theoretical framework to analyze social and spatial coordination in the specific case of location-based communication. In "Walking in the hybrid city: From micro-coordination to chance orchestration," Van den Akker starts from the premise that social encounters in location-based social networks, such as Foursquare, are neither wholly aleatory (such as playfully finding new experiences or places by pure chance), nor necessarily have a prior purpose (such as teleological navigation); they are instead domesticated by users into a surprising combination of both. As a result, he develops the new concept of "chance orchestration" to define ways in which location-based social network users continuously create ways to run into unexpected but desirable opportunities and encounters, presented by digitally augmented circumstances, such as spontaneous meetings with other people who check in at nearby locations or recommend locations nearby. He conceptualizes this idea of chance based on Lefebvre's (1991) theory of moments and de Certeau's (1988) theory of occasions, in order to argue that time is as important as space when analyzing location-based media.

In Chapter 3, "Direct video observation of the uses of smartphones on the move: Reconceptualizing mobile multi-activity," Christian Licoppe and Julien 
Figeac describe a method for video recording that uses a combination of camera glasses and mobile screen capture to gain new insights into the joint management of mobile communication and navigation through urban spaces, and more generally multi-activity. They use this method in an empirical study of the use of smartphones in transport situations, and specifically driving a car. Licoppe and Figeac are interested in how users distribute their attention between driving and mobile communication activities, particularly in terms of how users are either absorbed by or break away from the mobile phone screen and the street view. Their main argument is that this kind of rich method for capturing the practical accomplishment of everyday situated practices is necessary to make sense of how emerging mobile technology-mediated activities are incorporated into existing patterned social activities such as driving or commuting. Like the chapters by Ling and van den Akker, this chapter also suggests that there are new forms of situated practices involved in the management of attention, switching between temporal textures, and the coordination of everyday activities. Ultimately, their study might help to improve the design of mobile communication application interfaces for multi-activity settings by better understanding the complex temporal and spatial interactions performed by users as they switch back and forth between different mobility systems.

Driving while texting is certainly dangerous; navigating the desert on the USMexico border, however, presents other kinds of dangers. Moving from entertainment to border politics, from everyday routines to more unusual interventions, and from urban spaces to the remote desert, in Chapter 4 Fernanda Duarte investigates how location-aware technologies mediate power relations and have the potential to work as forms of political resistance and subversion. In "Rerouting borders: Politics of mobility and the Transborder Immigrant Tool," Duarte analyzes the case of the Transborder Immigrant Tool-a political/aesthetic intervention by the Electronic Disturbance Theater via an app designed to assist individuals navigate through the desert in the surroundings of the USMexico border. Again the aim is to show how there is no necessary imperative for a given technology to function only as a system of control and power (like Ling's Gesellschaftlichkeit), for in this case the technology of the Global Positioning System (GPS) can be made to work against its original purposes by empowering inventive and circumventive action. The app works by pointing out the safest routes and the position of water caches - accompanied by poetic readings to humanize the border experience - to demonstrate the political implications of using location-based media as activist tools. As such, the chapter opens up for further dialogue the political and territorial matters that ground the discussions not only about immigration policies and border barriers, but also about mobile locative technologies, their uses and meanings.

The second part of this book on "Performing location, place-making, and mobile gaming" offers studies of particular areas of practice that tie together location-awareness and mobility. This part shows the ways in which various users have appropriated mobile technologies to create new kinds of connections 
to locality and place-making, whether for purposes of belonging, attachment, nostalgia, and pleasure, or for more business-oriented commercialization and gamification. Here the arguments are part of a wider response in recent years to Marc Augé's notion of "non-place" (1995), which has sparked the revival of interest in how place-making occurs even in the most unexpected and seemingly inopportune locations. What seems like a homogenous, empty, copycat nonplace may in fact be full of meanings, memories, feelings, and personal experiences. Exploring these questions through the intersection of mobility, mobile communication, and locative media offers a new lens on the relation between movement, belonging, affect, feeling, attachment, and sensation, all of which are being played out in spaces of urban transit and mobile internet access, as well as through mobile gaming.

In Chapter 5, "Online place attachment: Exploring technological ties to physical places," Raz Schwartz uses the concept of "place attachment" to examine the tensions and implications of local connections between people and places. He uses Foursquare as a case study to investigate the digital elements that help to connect people to specific locations, and why people feel an intimate attachment to specific places by using location-based technologies. Incorporating offline experiences such as place-naming, ownership, and celebratory events, online place-making also includes extensive documentation, collective attachment, and mutual connection, all of which could be seen as performances of what Ling understood as digital Gemeinschaft.

Similarly in Chapter 6, "Location as a sense of place: Everyday life, mobile and spatial practices in urban spaces," Didem Özkul seeks to understand the various meanings a place takes for different people by exploring how sharing location information via mobile devices contributes to constructing different senses of places. Özkul does not restrict her analysis to the use of location-based media; rather, she takes into consideration several different ways mobile users talk about "place" and "location" in their everyday interactions with others and the city. In her interviews with smartphone users in the city of London (UK) she found out, among other things, that locational information is extremely relevant to construct a sense of place and build attachment to places in cosmopolitan cities, such as London. Rather than location being mere coordinates, empty of meaning, locational information is deployed in deeply felt ways. She concludes that contrary to the common belief that mobile communication technologies contribute to detaching people from place, location information actually (re)attaches people to places.

In Chapter 7, "Performing city transit," Taien Ng-Chan also addresses the fleeting movement of commuters through urban spaces, but takes a closer looks at the in-between spaces of city transit as a liminal space of everyday ritual, a performance site of urban contact, and a hybrid mobile space through the use of locative media. Drawing on notions of the experience of commuting as "gift time" and "equipped time," she uses her own auto-ethnographies and participant observation of riding the metro through the city of Montréal (Canada), to 
develop the notion of city transit as an in-between space of liminality and patterned ritual that may take on aesthetic qualities. Ng-Chan establishes how the use of mobile and locative media, particularly sound-based art, can be incorporated into the hybrid mobile space of being in transit to generate a kind of aesthetics of mobility. Locative mobile art can thus contribute to the "pleasures of making place" within the Montréal transit network.

While the previous studies argued that smartphones, location-based social networks, and the navigation of urban spaces contributed to place attachment, in Chapter 8, "Location-based gaming apps and the commercialization of locative media," Dale Leorke argues that newer commercial location-based mobile games (LBMGs) in fact diminish our capacities to connect to places and locations because of their strong commercial imperatives. Leorke examines commercial LBMGs that emerged after the launch of the iPhone and Android platforms. He presents two games, Life is Magic and Shadow Cities, to claim that LBMGs distanced themselves from their avant-garde origins in the locative art movement of the 2000s and are now driven by the commercialization of apps. As a consequence, newer LBMGs offer players fewer opportunities to interact with the surrounding physical space. The location-based elements of these games become thus subordinated to the profit-driven, commercial aspect of the mobile gaming industry. In contrast to Duarte's study of the Transborder Immigrant Tool and $\mathrm{Ng}$-Chan's emphasis on the aesthetics of mobility, here we see the opposite: An emptying out of spatial meanings and embodied practice in favor of rote activity that decreases meaningful place-making. Perhaps the revenge of Gesellschaft?

In Chapter 9, "Houses in motion: An overview of gamification in the context of mobile interfaces," Nathan Hulsey also discusses the commercialization of locative media and LBMGs. Hulsey starts from the same principle that the popularity of mobile phones has brought mobile gaming out of the realm of experimentation (and artistic innovation) and into the mainstream, especially in the case of corporate marketing and advergaming. The chapter examines gamification as the embedding of game mechanics into everyday activities such as shopping, mobile practice, energy consumption and spatial representation. Hulsey investigates the effects of gamified systems on everyday mobility, spatial practices and economy. He argues that game mechanics and mobile technologies work in concert with each other to recontextualize physical and social spaces while also allowing for profitable surveillance, data mining, and the use of predictive algorithms to manipulate behavior. Together these two chapters remind us that locational information can also empower commercial entities to perform place-making and attachment in new ways, as they harness mobile locative technologies to entice customers to engage with their stores, their brand, or their products. As Shklovski et al. (2009) suggested, what we are witnessing right now is the "commodification of location."

The last part of the book, "Mobile cities: Mapping, architecture and planning," moves out into the application of these ideas in urban planning, urban mapping, architectural simulation, and urban design theory and methods. Here 
there are truly exciting opportunities for new work to emerge out of a conversation between communications scholars, mobilities researchers, and these very different types of professional practice. The theoretical legacies of various forms of training bring multiple perspectives to bear, while the empirical grounding of architecture and design in the built environment demand that theory be conversant with realities "on the ground" so to speak. Yet mobile locative technologies bring a disruptive impetus to traditional methods and approaches, in part because they offer new opportunities for participation, collaboration, and dynamic temporalities for visual processing, annotation, and feedback circuits.

In Chapter 10, "Exploring locative media for cultural mapping," Peter Hemmersam, Jonny Aspen, Andrew Morrison, Idunn Sem, and Martin Havnør examine how mobile media can be used to read and write space. They explore the relations of locative and social media to the mapping and design of cities. For this purpose, they developed an urban mapping platform as an iPhone application called Streetscape, which explores the potential of using locative mobile media as a tool for developing participatory forms of collaborative cultural mapping in urban settings. In the chapter, they describe how the tool was tested with Norwegian students. They also suggest that Streetscape offers an experimental platform for thinking differently about how cities are designed, choreographed, and curated. Thus, like other chapters in this part, they offer not only theories of urban performance, but also new tools for using mobile locative interfaces in urban planning and design, and new methods for evaluating outcomes.

In Chapter 11, "Designing for mobile activities: WiFi hotspots, users and the relational programming of place," Michael R. Doyle argues that researchers should pay more attention to the physical configurations of the places where mobile technology use occurs. As such, he argues that place becomes more important as location-based mobile technology responds to the immediate context and configuration of the urban space. Doyle studied the use of free WiFi hotspots in Québec City, Canada in order to map the distribution of WiFi users throughout the city, characterize the main types of WiFi users, and unveil the logic beneath the physical characteristics of places that attracted these users. By focusing on spatial design and the potentials of architecture to influence WiFi use, he integrates the study of mobile media with urban design theory. His conclusions point toward the challenge of programming architectural space in ways that plug into the urban scale, while also conjoining the material structure with the immaterial digital networks that traverse it.

Some designers have already taken up one part of this challenge by creating new tools for situated simulation (sitsim), which can be deployed as part of a practice of participatory design and public visualization of new architectural projects. In Chapter 12, "The power of place and perspective: Sensory media and situated simulations in urban design," Gunnar Liestøl and Andrew Morrison present sitsim, an indirect augmented reality platform to be used in the planning of the new national museum being constructed in downtown Oslo, Norway. 
While traditional augmented reality applications have been used in architecture to visualize the past, this new app allows users to visualize what the museum will look like in the future and how it will relate to surrounding buildings and urban space. They trialed the app in early 2012 and found that the immediate benefit for users with this form of representation and mediation is the active combination of two perspectives, the physical and the computer generated, at the user's location and from the user's subjective point of view. In contrast to traditional two-dimensional sectional drawings, or even three-dimensional building information models, the sitsim offers a dynamic situated perspective, which can also be used for annotation of collective perceptions. As such, they demonstrate how place, location and perspective change with the development of mobile augmented reality in urban planning.

Closing this part, and building on all that has been said in the preceding chapters, Ole B. Jensen addresses the need of urban planners and designers to master networked technologies. In "The will to connection: A research agenda for the 'programmable city' and an ICT 'toolbox' for urban planning," Jensen discusses the concept of an "ICT toolbox" for urban planners and designers, and explores how such training is being implemented in Aalborg, Denmark. Jensen considers how contemporary cities are embedded with mobile and locative technologies, and how designers and urban planners need to embrace, engage with or resist these technologies in order to understand urban spaces. As we enter a new period of what he calls "programmable urbanism," the designer's or planner's ICT toolbox should include not only hardware such as GPS trackers, CCTV, heatsensitive cameras, RFID tags, GIS maps, and 3D dynamic visualizations, he suggests, but also the necessary "theoretically informed knowledge about the social and cultural embedding of these technologies" (Jensen, this volume). We hope that this volume has provided precisely such theoretically informed knowledge as we embark on this transdisciplinary journey to develop new methodologies for studying mobility, mobile communication, and locative media.

As we ourselves cross boundaries in search of the adjacent possible we find ourselves drawn to the work of artists who have become theorists, or theorists who have become artists, in either case tinkering with the edges of technologies, ideas, and practices. In Chapter 14, "Restless: Locative media as generative displacement," which serves as an epilogue to the book as a whole, we invited ground-making artist (and new-edge locative media theorist) Teri Rueb to discuss her work with locative media, which began with the 1999 piece "Trace" - one of the first geo-annotated mobile art projects to use GPS coordinates embedded in the landscape to access a locative sound installation. Drawing on process theory, her work explores what she calls "the whole space of body-site-movement interaction" with "location-sensitive information or responses" (Rueb, this volume). She develops the concept of mobile experience to refer to everyday experience that is mediated by location-sensing technologies. Weaving concepts from process philosophy and affect theory through a narrative of her own locative media practice as it has evolved over the past 
fifteen years, she reveals locative media as a form of generative displacement, where the body is reconfigured in its relation to itself (the sensorium), to the environment (through both physical and cultural perception of place), and to others, including human and non-human realms.

One of our key aims with this collection is to leverage the adjacent fields of mobilities, mobile communication and locative media in order to open up more theoretically nuanced and methodologically enriched understandings of the contemporary moment in the formation and performativity of hybrid space. We hope readers will come away with an enhanced multi-layered perspective on key topics including coordination, navigation, social cohesion, multi-tasking, place-making, the significance of location, the implications of mobile gaming, mobile mapping, architectural simulation, and new tools for urban planning and design. At the same time we hope to have sparked an interest in mobile locative art, the aesthetics of mobility, and the politics of mobile mediations. And above all, we hope to have cleared a place for transdisciplinary conversations, for new methodological practices, and for the invention of new tools for interaction and intervention by which we might generate the next confluence of adjacent possibilities.

\section{Note on terminology}

The chapters that follow will use the terms cell phone and mobile phone interchangeably, depending on the social context. Interestingly, the US term "cell phone" (from "cellular") puts emphasis on the physical and spatial infrastructure through which the telephone connection is transmitted, a series of "cells" connected via transmission towers; while "mobile phone," a term more widely used in many other parts of the world and often shortened to "mobi," puts an emphasis on the capacity of the user to be mobile while using the phone and the mobility of the phone itself.

\section{References}

Adey, P., D. Bissell, K. Hannam, P. Merriman and M. Sheller (eds.) (2014) The Routledge Handbook of Mobilities, London and New York: Routledge.

Augé, M. (1995) Non-Places: Introduction to an Anthropology of Supermodernity, London: Verso Books.

Blast-Theory and The-Mixed-Reality-Lab (2001) Can you see me now? Sheffield, Rotterdam, Tokyo, Barcelona.

Brown, B., N. Green, and R. Harper (2002) Wireless World: Social and Interactional Aspects of the Mobile Age, London: Springer Verlag.

Büscher, M., J. Urry, and K. Witchger (2010) Mobile Methods, Abingdon/New York, NY: Routledge.

Campbell, S. W. (2013) Mobile media and communication: A new field, or just a new journal? Mobile Media \& Communication, 1(1): 8-13.

de Certeau, M. (1988) The Practice of Everyday Life, Minneapolis, MN: University of Minnesota Press. 
de Souza e Silva, A. and J. Frith (2010) Locative mobile social networks: Mapping communication and location in urban spaces, Mobilities, 5(4): 485-506.

Edensor, T. (2011) Commuter: Mobility, rhythm, commuting, in T. Cresswell and P. Merriman (eds.), Geographies of Mobilities: Practices, Spaces, Subjects, Farnham and Burlington, VT: Ashgate, 189-204.

Elliott, A. and J. Urry (2010) Mobile Lives, New York, NY/Abingdon: Routledge.

Fincham, B., M. McGuinness, and L. Murray (2010) Mobile Methodologies, Farnham: Ashgate.

Gergen, K. (2002) The challenge of absent presence, in J. Katz and M. Aakhus (eds.), Perpetual Contact: Mobile Communication, Private Talk, Public Performance, Cambridge: Cambridge University Press, pp. 227-241.

Goggin, G. (2013) Youth culture and mobiles, Mobile Media \& Communication, 1(1): 83-88.

Goggin, G. and R. Wilken (2012) Mobile Technology and Place, London: Taylor \& Francis.

Gordon, E. and A. de Souza e Silva (2011) Net Locality: Why Location Matters in a Networked World, Boston, MA: Blackwell Publishers.

Graham, S. and S. Marvin (2001) Splintering Urbanism: Networked Infrastructures, Technological Mobilities and the Urban Condition, London/New York, NY: Routledge.

Habuchi, I. (2005) Accelerating reflexivity, in M. Ito, D. Okabe, and M. Matsuda (eds.), Personal, Portable, Pedestrian: Mobile Phones in Japanese Life, Cambridge, MA: MIT Press.

Hannam, K., M. Sheller, and J. Urry (2006) Mobilities, immobilities and moorings, Mobilities, 1(1): 1-22.

Harper, R., L. A. Palen, and A. S. Taylor (2005) The Inside Text: Social, Cultural and Design Perspectives on SMS, Dordrecht: Springer.

Hemment, D. (2004) Locative Dystopia 2, available at: www.makeworlds.org/node/76.

Hemment, D. (2006) Locative arts, Leonardo, 39(4): 348-355.

Iverson, H., M. Sheller, and L. Aceti (eds.) (2014) L.A. Re.Play, Special Issue of Leonardo Electronic Almanac, forthcoming 2014.

Jensen, O. B. (2009) Flows of meaning, cultures of movements - urban mobility as meaningful everyday life practice, Mobilities, 4(1): 139-158.

Jensen, O. B. (2010) Negotiation in motion: Unpacking a geography of mobility, Space and Culture, 13(4): 389-402.

Johnson, S. (2010) Where Good Ideas Come From: The Natural History of Innovation, New York, NY: Riverhead Books.

Katz, J. and M. Aakhus (eds.) (2002) Perpetual Contact: Mobile Communication, Private Talk, Public Performance, Cambridge: Cambridge University Press.

Kaufmann, V., M. Bergman, and D. Joye (2004) Motility: Mobility as capital, International Journal of Urban and Regional Research, 28(4): 745-756.

Kellerman, A. (2006) Personal Mobilities, London: Routledge.

Keyani, P. and S. Farham (2005) Swarm: Text messaging designed to enhance social coordination, in R. Harper, L. Palen, and A. Taylor (eds.), The Inside Text: Social, Cultural, and Design Perspectives on SMS, Dordrecht: Springer, pp. 287-304.

Larsen, J., J. Urry, and K. W. Axhausen (2006) Mobilities, Networks, Geographies, Farnham: Ashgate.

Lefebvre, H. (1991) The Production of Space, Malden, MA: Blackwell Publishers. 
Ling, R. (2004) The Mobile Connection: The Cell Phone's Impact on Society, San Francisco, CA: Morgan Kaufman.

Ling, R. and B. Yttri (1999) Nobody sits at home and waits for the telephone to ring: Micro and hyper-coordination through the use of the mobile telephone, in J. Katz (ed.), Perpetual Reachability Conference, New Brunswick, NJ: Rutgers University.

Ling, R. and B. Yttri (2002) Hyper-coordination via mobile phones in Norway, in J. Katz and M. Aakhus (eds.), Perpetual Contact: Mobile Communication, Private Talk, Public Performance, Cambridge: Cambridge University Press, pp. 139-169.

Packer, J. (2008) Automobility and the driving force of warfare: From public safety to national security, in T. Sager and S. Bergmann (eds.), The Ethics of Mobilities, Aldershot: Ashgate, pp. 39-64.

Paulos, E. and E. Goodman (2003) The Familiar Stranger: Anxiety, Comfort, and Play in Public Spaces, Berkeley, CA: Intel Research.

Pearce, K. E. (2013) Phoning it in: Theory in mobile media and communication in developing countries, Mobile Media \& Communication, 1(1): 76-82.

Puro, J. P. (2002) Finland, a mobile culture, in J. Katz and M. Aakhus (eds.), Perpetual Contact: Mobile Communication, Private Talk, Public Performance, Cambridge: Cambridge University Press, pp. 19-29.

Putnam, R. (2000). Bowling Alone: The Collapse and Revival of American Community, New York, NY: Simon \& Schuster.

Rice, R. and J. Katz (2003) Comparing internet and mobile phone usage: Digital divides of usage, adoption and dropouts, Telecommunications Policy, 27(8/9): 597-623.

Rueb, T. (1999) Trace, Banff, Canada.

Rueb, T. (2007) On Itinerant, in P. Harrigan and N. Wardrip-Fruin (eds.), In Second Person: Role-Playing and Story in Games and Playable Media, Cambridge, MA: MIT Press, pp. 273-278.

Sheller, M. and J. Urry (2003) Mobile transformations of "public" and "private" life, Theory, Culture \& Society, 20(3): 107-125.

Sheller, M. and J. Urry (2006a) The new mobilities paradigm, Environment and Planning A, 38(2): 207-226.

Sheller, M. and J. Urry (eds.) (2006b) Mobile Technologies of the City, London/New York, NY: Routledge.

Shklovski, I., J. Vertesi, E. Troshynski, and P. Dourish (2009) The commodification of location: Dynamics of power in location-based systems, Proceedings of the 11th International Conference on Ubiquitous Computing, Orlando, FL: ACM.

Thrift, N. (2008) Non-Representational Theory: Space, Politics, Affect, New York, NY: Routledge.

Tönnies, F. (1965) Gemeinschaft and Gesellschaft, in T. Parsons, E. Shils, K. D. Naegele, and J. R. Pitts (eds.), Theories of Society, New York, NY: The Free Press, pp. 191-201.

Turkle, S. (2011) Alone Together: Why We Expect More From Technology and Less From Each Other, Philadelphia, PA: Basic Books.

Tuters, M. and K. Varnelis (2006) Beyond locative media: Giving shape to the internet of things, Leonardo, 39(4): 357-363.

Urry, J. (2003) Social networks, travel and talk, The British Journal of Sociology, 54(2): $155-175$.

Vukov, T. and M. Sheller (2013) Border work: surveillant assemblages, virtual fences, and tactical counter-media, Social Semiotics, 23(2): 225-241. 
This page intentionally left blank 
Part I

Rethinking cohesion,

coordination, and

navigation 
This page intentionally left blank 


\section{Mobile phones and digital Gemeinschaft}

\section{Social cohesion in the era of cars, clocks and cell phones}

\section{Rich Ling}

Technology can be seen as the subject for a type of Cubist painting. When viewed from one perspective we get one image, but when we look at the other profile, something else comes up. In this chapter, I want to look at technologyin particular the mobile phone and the car-from two different positions. On the one hand, these two technologies are a part of the broader drift toward what Ferdinand Tönnies (1965) called Gesellschaft. That is, they facilitate the rationalization of society and move us away from the traditional and the intimate sphere. However, I argue that they are also used in the maintenance of a residual Gemeinschaft. That is, we use them to stay in contact with people who are important to us. We use technologies such as the car and the mobile phone to build and maintain cohesion within Gemeinschaft. While these technologies often arose in the name of rationalizing society, i.e., Gesellschaft, I suggest that they are repurposed for our social needs. In a sense this reverses Habermas in that it is the colonization of the system by the lifeworld (Habermas 1985; Fairtlough 1991).

I also argue that the use of the car and the phone is not simply our own choice but rather, for many people, they are a necessarily patterned part of life over which we have little choice. While it is clear that people living in tight city cores with well-developed public transport do not need a car, for many people living in the suburbs of North America, Europe, Australia and portions of Asia, there is no reasonable alternative. Using the car is a structured part of society. The mobile phone is also taking on a similar status. That is, in most cases, we do not have the ability to choose to not have a phone or own a car. This is because it is expected of us by those with whom we interact. While the case of the car falters when considering transportation in tight urban centers, the mobile phone is used for coordination in the core cities, in the suburbs and indeed in rural areas. We use the mobile phone to coordinate ourselves with one another across a broad spectrum of transportation forms.

That said, the car - particularly in the suburban context — and the phone have an interesting symbiosis. The car facilitated the development of the vast suburban reaches where there was also the development of truck-based supply systems to remote shopping centers. There was not, however, a corollary system with 
which to coordinate interaction. It was not until the mobile phone provided individual addressability that the broad mass of people could interact when we were away from the landline telephone system. Thus, the car provides the transportation and the phone gives us the ability to coordinate. We can use the phone to organize our business meetings but we can also use it to chat with a friend and to arrange a cup of coffee. Playing on Tönnies' (1965) ideas, the use of the mobile phone for the maintenance of friendship is what might be called the digital Gemeinschaft. In this chapter I examine the tension between Gemeinschaft and Gesellschaft and then describe how mobile communication and car-based transportation are being used to maintain our intimate social spheres, or what Tönnies would call Gemeinschaft. Finally, I examine the synergy between car-based transportation and mobile communication.

\section{The rise of massive logistical systems: communal vs. associative society}

Tönnies (b. 1855, d. 1936) was interested in the contrast between traditional and industrialized society that he has characterized as Gemeinschaft (community or fellowship) and Gesellschaft (rational corporate society). Gemeinschaft is kinship, neighborhood, friendship, intimacy, folkways, and mores. By contrast, Gesellschaft emphasizes self-interest, logic, competition, formal interaction and contractual arrangements, and business. Gemeinschaft focuses on group-oriented interactions while Gesellschaft directs itself toward individualistic calculation. Tönnies says that the values of kinship, friendship, and the private sphere of Gemeinschaft are being replaced by individualism and commercialism of Gesellschaft. He suggests that in Gesellschaft, there is the potential to organize everincreasing portions of society in its image. ${ }^{1}$

Working in somewhat the same vein, James Beniger examines how the increasing speed of material processing during industrialization (that is during the drift toward Gesellschaft) precipitated a crisis of control. In turn, this prompted the need for information control technologies. Beniger shows how control systems are the foundation for bureaucratic organization, telecommunication, and transportation (Beniger 1986: 432). Put into Tönnies' terms, this is the advance of Gesellschaft.

Moving into the twentieth century, there were ever-wider possibilities for large-scale production where Gemeinschaft came into the shadow of Gesellschaft. This could, for example, be seen in the development of production processes. Drawing on the experience of Eli Whitney, Henry Ford and Charles Sorensen first developed moving assembly lines (with task specialization as opposed to crafting of products) to produce Model-T Fords (Sorensen 1956). Following this there was "just in time" production most famously in the production of Toyotas (Shingo and Dillon 1989) i.e., tightly controlled production that integrates the assembly process with a myriad of feeder producers to reduce the need for inventory backlogs. Unlike the Gesellschaftlich crafting of products by 
a single individual, this type of production demands a tight scheduling of work that is correlated with fluctuations in demand (Kumar 1995). As discussed by Beniger (1986), these production processes require extremely sophisticated control systems. The same drift toward regimentation and rationalization goes through other phases of our lives, in everything from how we shop, to how we are educated, how we address our individual needs, and how our private activities are being catalogued.

This story line suggests that Tönnies (1965) was right. The communal Gemeinschaft was being thumped by logic and contractual nature of Gesellschaft. According to Beniger (1986), all of this has had implications for the situation of the individual. The push toward standardization is seen in many of our interactions with jobs, education, shopping, and other formal dimensions of life. Further we are seeing the intrusion of the control society into the private sphere in the form of surveillance (Weller 2012). I argue however, that we repurpose technologies to retain the Gemeinschaft.

\section{Digital Gemeinschaft: personal technologies as control revolution spillovers}

Is there any hope for the individual, or for the small group of friends and family in the drift toward Tönnies' Gesellschaftliche dystopia? While there are undeniable efficiencies to be realized by the machining of the production process, there does not seem to be space left for a warm, welcoming interpersonal life (Giddens 1991: 89). I suggest that there is, in all of this, the will for non-rationalized social interaction and further that technology serves an essential role in this.

The evidence suggests that Gemeinschaft has persevered, aided by technologies that arose in the name of rationalization. Our nearest family, our friends, and our neighbors are important even in the face of the centrifugal forces often associated with information and communication technologies (ICTs) (Hampton and Wellman 2003). These people are important for care giving and providing succor. In addition to the local network, there are family and friends spread across the city.

The typical network community in North America consists of a small number of densely-knit immediate kin and a larger number of sparsely-knit friends, neighbors, workmates, and extended kin.... Neighborly relationships remain important, but as a minority of ties within the overall network community.

(Hampton and Wellman 2002: 279; see also Gordon and de Souza e Silva

2011: 110)

This web of connections is what Peter Berger and Hansfried Kellner call the "nomos," a concept that describes a richness of social ties (1964: 7). They note that in the ongoing production of the group there is the need to build up a 
common sense of the group, its traditions, and its notion of itself. One of the mechanisms through which this is done is various types of ritual interaction either face-to-face (Collins 2004) or, in some cases, via mediation (Ling 2008). These considerations become a part of the common baggage of the group. ${ }^{2}$ They form a common ideology (Ling 2012) and it is through these ideologies that the group is maintained.

The nomos is perhaps most elaborately developed in the family (Ling 2006). The family is a particularly segregated group where there is a common project of fostering children and providing care giving. It is also a context where there is the expectation that there are special rituals and traditions that have the effect of bonding the members. There are special routines for meal times and special inside jokes that only the family members know. It is in this context that the nomos, or what Tönnies would call Gemeinschaft, usually is the most fully developed. It is also in this sphere that we use what I call technologies of social mediation to maintain cohesion in the face of the rationalizing drift of society.

\section{The tension between Gemeinschaft and Gesellschaft}

There is a tension between the forces of Gesellschaft that push us in the direction of increasing isolation and the legacy of Gemeinschaft that has the opposite effect. That said, the nomos or Gemeinschaft has staying power. In addition, it is using mediation technologies that were perhaps originally conceived of as industrial control technologies, to help maintain cohesion. In the words of Hampton and Wellman, "Private and parochial life continues to be important, with kin providing a stable core of broadly supportive relations and neighbors providing immediate access to tangible goods and services" (2003: 278). Gemeinschaft is being attended to in new ways. It is finding new channels through which it is practiced, but it is still vital.

Mediated interaction has a role and indeed there is an odd jujitsu in all of this. The very technologies that serve the purposes of capitalism are often reconceptualized to afford the needs of individuals and their social spheres. The mobile phone was developed and first used in the name of Gesellschaft. It arose out of rationalizing impulses associated with other dimensions of information technologies. It was the child of the telephone system that, in its original format, was seen as a tool of industry and commerce. To even a greater degree the telegraph was framed on this pattern. Thus, it is not surprising that the first applications of the mobile phone were in the service of business (Johannesen 1981). This phase did not last long, however. The price of mobile phones and the cost of subscriptions fell and by the early 1990s in many countries, users that are more "nontraditional" were becoming common. Teens calling their boy/girlfriends and adults calling partners and friends to exchange light banter became increasingly commonplace. This suggests that there is a somewhat dislocated, but enduring (and perhaps endearing) Gemeinschaft that among other things draws on the functionality of mobile communication. 
As the technology developed the cost of production fell and as subscription forms were better suited to the needs of families and individuals, the mobile phone became a feature of daily life. We increasingly use the mobile phone to coordinate (and micro-coordinate) daily life (Ling 2006). Indeed, it is within the closest sphere of friends and family that we use the mobile phone (Ling, Bertel, and Sundsøy 2012). In an analysis of mobile phone use in Norway, ${ }^{3}$ half of all calls and texts go to fewer than five different people. Younger people have somewhat larger social networks and elderly people have smaller circles. In addition, the analysis shows that the people with whom we talk are not too far distant. For example, the analysis of 26 million calls made in Norway shows that a quarter of the calls and texts were to people with their phone registered in the same postal zone. Half were within fourteen kilometers. Thus, while there is a sense that ICT opens up the broad world, the data from Norway show that Norwegians actually use it to stay in touch with a small group of people who are physically near at hand or within the radius of a short drive. Most calls and texts are to a limited number of people who live within a "city-sized" area. That is, we use the mobile phone to cultivate the Gemeinschaft $t^{4}$ (Ling et al. 2012). These considerations describe the durability of Gemeinschaft. It is an important link to our nearest circle of friends and family. The landline phone was also used for this purpose, but the great advantage of the mobile phone is that it makes each of us individually available and thus affords much better person-to-person contact.

The mobile phone and other forms of direct interpersonal contact help us to create and maintain cohesion. In the words of a teen from a focus group in the US, "The best thing [about the mobile phone] is that it's so convenient and you can just talk to people all the time, and like even if you're not at home" (Lenhart et al. 2010: 74). Others have found as much. Kenichi Ishii (2006) has reported that it helps people to maintain bonds in the intimate sphere and Christian Licoppe (2004) has discussed how the device gives us direct, unhampered "connected presence." This is to say that a digitally supported version of Gemeinschaft is an integral part of our lives. The mobile phone is used for both expressive and instrumental ends. It lets us coordinate our daily routines and make them more effective; in addition, it allows us to call a friend for a quick chat when we are down or when we have some exciting news. Using the paraphrase of Mizuko Ito and Daisuke Okabe (2005) mobile phones allow us to give one another a digital clap on the back to let them know we are there. All of this ties us more closely together and it is these ties that give the social group resilience.

To say that the mobile telephone is a part of the digital Gemeinschaft does not necessarily mean that it is only a benign and cozy addition to daily life. It can be used to check on family and friends in a variety of situations, but it can also be used to badger them. Just as rational bureaucracies can push us unwillingly in one direction or the other, so can the demands of local traditional power centers, be they the strictures of the priest or the fear of the local wag. Parents can call their teens at inopportune moments in the eyes of the teens, and vice versa. 
There is the potential for intimidation in the web of mutual expectations associated with life in the Gemeinschaft. It is not necessarily a utopia, but can be populated by local tinhorned kingpins to whom we need kowtow.

In spite of this if we are to be considered a legitimate family member, friend or even acquaintance, we have to play by the rules. We have to be available to others as needed and we have to be there to fulfill our role in the firmament of Gemeinschaft. It is naive to think that either Gemeinschaft or Gesellschaft is only a utopia; both have power structures that will exercise their initiative vis-àvis the individual.

\section{Technologies of social mediation: technologies through which we carry out sociation}

Up to this point, I have been generally talking of the mobile phone as a device of the individual. There is the common notion that it is a gadget in our own personal service. It is something from which we, individually, gain utility. I now want to make the transition into talking of the mobile phone, as well as other technologies, as something that provides utility to a group. I am interested in thinking of mobile phones (as well as, for example cars, clocks, and social networking sites) as technologies through which we carry out social interaction.

Considering the mobile phone, it is increasingly taken for granted. We are no longer surprised that another person has a mobile phone. We may be envious that they have the newest model or a particularly stylish brand, but we are not surprised. The mobile phone is also taken for granted in a more social sense. That is, we expect others to be available via their mobile phones. This describes the social dimension to ownership and use since the functionality of the device is not only a benefit to the individual who owns a particular handset, indeed a handset without the network and contact with other users has very little value. The value is in the ability to contact one another (in addition to the access to information). It is in this way that the mobile phone has become what I call a technology of social mediation (Ling 2012).

I have defined technologies of social mediation as "legitimated artifacts and systems governed by group-based reciprocal expectations that enable but also set conditions for the maintenance of our social sphere" (Ling 2012: 7). Playing off Durkheim's notion of social facts (1938), the use of social mediation technologies is not a matter of personal choice but rather it is an assumed part of social interaction. There is a structural imperative to their use. We ignore them at the peril of being outside the common forms of interaction. Durkheim says that social facts: "are imbued with a compelling and coercive power by virtue of which, whether he wishes it or not, they impose themselves upon him." He continues "it asserts itself as soon as I try to resist" (Durkheim 1938: 51). Further, he writes:

They come to each one of us from outside and can sweep us along in spite of ourselves. If perhaps I abandon myself to them I may not be conscious of 
the pressure that they are exerting upon me, but that pressure makes its presence felt immediately [when] I attempt to struggle against them

(Durkheim 1938: 53)

There is a difference between technologies of social mediation and standalone technologies. While differences are not absolute, I see social mediation technologies as literally that, i.e., technologies that mediate sociation. This definition is broader than is often used when speaking of, for example, electronic media (Livingstone 2008). In the study of communication, mediation is seen as an encoding of information that allows for its transfer. A scene is, for example, taken up by a video camera, digitized and sent via the television or the internet to others. In a broader sense a medium can be seen as something that intervenes between actors and which they can use to carry out their social commerce. In this broader sense it can be a device used to coordinate us with others, allowing us to communicate with them or it can be used to transport us to them. In this broader definition, the car, the clock, social networking sites, as well as the mobile phone are all social mediation technologies.

The key to understanding fully formed social mediation technologies is that we expect them of one another. They are sometimes easiest to see in their absence. I have drawn on a comment by James Katz in this context, namely that you become a problem for me if you do not use a social mediation technology (Ling 2012). If, on the spur of the moment, I want to invite a friend from across town to dinner but he or she does not have a mobile phone or has a car that is on the blink, then the event becomes stranded. I would have to make some extraordinary effort to get in touch if it is their mobile phone on the blink (and they are not near their PC for example). If it is their car that is the problem (and they live in a city where there is little public transport) then I would have to drive to get them. Either way, they become a problem for me when their devices are not in order.

Beyond this, social mediation technologies have four characteristics. First, we need to have the perception that there is a critical mass of users in our group or in our society. If we are, for example, the only person with a fax machine, there is little point. However, as more people get one, utility of the device increases. At some point, there is a transition. Rather than being surprised if another person has one, we come to expect it. Finally, at some point we begin to rely on it and, following the Katz principle, it is awkward with someone who does not have one since we have structured our lives around the functionality of everyone having fax machines. The important point is that as a social mediation technology moves into society and gains the mantle of criticality, it comes to structure interaction. Max Weber's metaphor of the iron cage is evocative here. He suggests that social constructions often are originally to "only lie on the shoulders of the 'saint like a light cloak, which can be thrown aside at any moment." "However as they work their way into society, they become an iron cage that we cannot ignore (Weber 1930: 181). 
Second, in addition to requiring the perception of a critical mass, technologies of social mediation carry with them legitimation structures that facilitate adoption. As the adoption process takes place we construct justifications, or what Berger and Luckmann call legitimations (1967). Berger and Luckmann suggest that our mutual agreement regarding a technology "requires legitimation, that is, ways by which it can be 'explained' and justified" (1967: 79). These are constructed by both the individuals who use them and the commercial interests that push for adoption. There can be counter legitimations that sing the praises of the previous incumbent (horse-based vs. car-based transportation for example).

Third, as a technology becomes embedded in society, it changes the broader social ecology. The metaphor of a niche is useful in conceptualizing this dynamic. A niche is a position in the ecology that is generally occupied by a species. The boundary of the niche is determined by the different constraints met by the species and, all things being equal, a species eventually establishes an equilibrium population. It is possible to use this metaphor to describe the "population" of various human artifacts (Boulding 1955). Using this approach we can also think that there are open niches such as cats in Indonesia, rabbits in Australia, starlings and tamarix in North America, and numerous other examples that illustrate the often unfortunate impact of introducing new species into a biome (Money and Hobbs 2000). These invasive species show that when a new "species" starts to occupy an open niche, or one that is occupied by a weaker competitor, they can also change the boundaries of the niche and reform the broader social environment. In the biological world, the introduction of king crabs to the Kola Peninsula has resulted in changes in the marine ecology since they out-compete the existing species. In a similar way, the clock is better suited to the current socio-technical assemblage of society than is the sundial. The same can be said of the car vs. the horse and the mobile phone vs. the landline phone. As a result of this society has directed monies away from sundial production, buggy whips and telephone poles. It is clock towers, parking lots, and mobile phone exchanges that get the resources.

Finally, as has been alluded to, we have reciprocal expectations. That is, we take for granted that others have and use a particular technology. When there is a critical mass of use and the technology is embedded in society with the appropriate legitimation processes, society becomes structured around the use of the technology. Normal interaction assumes our use of the technology. Mechanical time-keeping occupies such a position. We could, for example agree to have a business meeting when the sun is at its highest point (and check this by looking at the shadows), or agree to meet clients at about half-way through the morning. Clearly, these approaches are far too loose. In addition, they would not fit into a society that is structurally aligned to the system of clock-based time-keeping. Thus, the technology enables our activities, but it also constrains use. 


\section{Transport, coordination and communication in the digital Gemeinschaft}

It is worth thinking about one last dimension of social mediation technologies and their role in the maintenance of the Gemeinschaft and for that matter in the Gesellschaft, namely that technologies can develop a symbiotic relationship. Not only do the technologies become a structural necessity, but also that particular technologies each of which is a structural necessity, enter into a symbiotic relationship with other structurally necessary technologies. There is the development of this in the case of the car and the mobile phone.

Car-based mobility - along with the structure of the suburbs - is for many one of the central factors in contemporary society since we use the car as a means with which to carry out social interactions. It is, indeed a social mediation technology. There is a critical mass, there are legitimation structures and it has dramatically rearranged the social ecology. Finally, for many people there is a reciprocal expectation that they are able to transport themselves. Car-based transportation is a structural exigency for people who live in one portion of the city, work in another and whose children have school and free-time activities in yet another direction. For people living in the suburbs of North America, Europe and Australia the car-based urban and suburban expanses are a fixture of life for many people (Hayden 2003). Mimi Sheller and John Urry say that "multiple socialities, of family life, community, leisure, the pleasures of movement and so on, are interwoven through complex juggling of time and space which car journeys both allow, but also necessitate" (Sheller and Urry 2006).

\section{The dual character of cars}

There are two dimensions to the use of technologies such as cars: one is Gemeinschaftlich and the other is Gesellschaftlich. In very real ways, they facilitate the tendency toward rationalization, toward Gesellschaft. The car and the truck/ highway-based logistics have resulted in the vast shopping centers that allow companies to rationalize marketing (Foster 1983). The size of the outlets means that they rely on serving a large catchment area, that means that the customers necessarily must drive (or in some rare cases take a bus) to the superstore. At the same time these large actors enjoy benefits of scale (Short 2006: 54, 128). They can demand better prices from their suppliers and in some cases have access to products that are denied to other smaller actors. Further, their advantages of scale and the fact that the can provide a type of "one stop shopping" means that they out-compete the local independently operated stores. Beyond merchandizing, the car-based system of transportation is also deeply embedded in the way that we seek work and in the geographic flexibility of workforce that can move across a metropolitan area as the need for jobs shifts from one to the other location (Ehrenreich 2008).

The car also has a Gemeinschaftliche dimension. It allows us to socialize and to seek out people with whom we share interests. We drive to the meeting of a 
local club or we drive to visit our sick mother. Much of social interaction also takes place with friends and family who live further afield. It is also clear that the automobile itself is the location for other types of social association. We use the car for "joy-riding" and "drunk driving" (Flink 2001: 159). When describing Middletown in the late 1920s, Robert Stoughton Lynd and Helen Merrell Lynd (1929) described the use of the car for what they primly called "courting" —or in the context of this paper what we might call the radical pursuit of Gemeinschaft. They were followed by others who discussed the same phenomena using more explicit terms (Bailey 1989: 159-161). Thus, the car serves the purpose of Gemeinschaft in that it transports us to our social engagements and it can be used for other, more private, forms of sociation.

\section{Symbiosis between the car and the phone}

The car and the mobile phone have moved into society and, each in its own right, have become a technology of social mediation. They have gained a critical mass, developed legitimation structures and our adoption of the technology has resulted in changes in the ecological system in which they have operated. Finally, we also have reciprocal expectations with regards to our mutual use of the technologies.

There are also obvious synergies between these technologies in that the one facilitates the use of the other. More specifically, the mobile phone facilitates use of the car since it adds a dimension of flexibility and coordination to the use of carbased transportation. The car, in its time, enabled the development of the vast suburbs. It did so, however, without a well-developed way to coordinate our movements with others once we were on our way and away from a geographically fixed landline telephone. The car-based transportation system grew without the corollary ability to individually coordinate interaction (Elliott and Urry 2010; Ling 2012). As people moved around the vast urban and suburban areas, they needed a system for coordinating meetings and social interactions. Until the mobile phone, time and location served this purpose. We could not change the temporal or geographic coordinates of our meeting since we, and our eventual meeting partners, were effectively incommunicado while in transit. The lack of direct real-time communication (Townsend 2000) added an element of chance when making arrangements. The car gave us personal transportation and expanded the range of movement, but it did not give us personal addressability. This was the role of the mobile phone. It brought us flexibility and resilience in planning daily life.

There is a symbiosis between these two. Indeed Lee and Lee (2012) find that expenditures on telecommunications and cars are generally complementary. The mobility of the mobile phone means that the car and the phone can meld into a single coordination/transportation complex that facilitates our movement in contemporary society. Together they result in a more finely tuned transportation system since people can coordinate their movements and their transportation needs. This potential was seen early in the diffusion of the mobile phone. A 
young teen girl, Lydia, was interviewed in the late 1990s about her use of the mobile phone. ${ }^{5}$ Lydia did not own one, but she could borrow one when she went horseback riding after school. She said that she used it to call her parents when she was done riding and they could come to get her. Rather than the parents arriving early and having to wait while other tasks were put on hold, or arriving late while their daughter shivered in the winter temperatures after riding, the family solved the situation by letting Lydia use the "family" mobile phone. Since that time, literally all teens and adults have procured their own personal phones. This diffusion means that they can use them in just such cases. When the teen is done with soccer practice or when the family has to coordinate shopping with other tasks that are spread across the landscape, the mobile phone is there.

There is a tension between the drift toward Gesellschaft and the residual but resistant Gemeinschaft. In general, ICTs and also the car/truck-based transportation system is pushing us in the direction of Gesellschaft. On the one hand, the movement/coordination system has a clear role in the ever-increasing Gesellschaftliche nature of society. Mobile communication lets us work out complex logistics of meetings, delivery and coordination. We can be at a remote workplace (i.e., a carpenter building a house) and change the delivery times for different components that need to be used in the construction process. We can be salespeople juggling meetings and checking the credit rating of an eventual purchaser before driving to a meeting with a new supplier. We can also use the phone and the car to arrange a Gemeinschaftliche picnic with friends and send one another text messages as to who will buy the watermelon and who should make some fried chicken (Fischer 1992; Rakow 1992). The car, now supplemented by the mobile phone, is becoming a structurally embedded element in our organization of daily life.

\section{Conclusion}

Mobile communication is both a control technology and a technology that facilitates the Gemeinschaft. It is also being woven into the very structure of daily life. It gives us a freedom and it makes our coordination more efficient, but from a social perspective, we must have a mobile phone. We accept it at the personal level because it facilitates our lives. Not to have it, however, is to not completely participate in society. It has become a part of the very structure of society.

Obviously, the technologies will continue to be developed in order to push us in the direction of greater Gesellschaft. We only need to look at the black wizardry of ICT in the finance world that pursues profits in the nanosecond. Is there the rationalization of interaction that is not really based on tradition and kinship, neighborhood, friendship, intimacy, folkways, and mores? Are we simply fooling ourselves that Facebook allows us to be empathetic with all our "friends" while at the same time we are given more time to administer our lives?

I choose to hope that there is a reservoir of Gemeinschaft and that some of the interaction is genuine. I choose to hope that we really care about one another and 
that we appropriate the technologies in order to be a better parent, partner, or friend. It may be that I am fooling myself. It may be that I have simply bought into the gadget fetish that is otherwise being used to squeeze more profits out for the owners of derivatives and hedge funds but without immediate social benefit. I may be desperately wishing for this kind of contact and grasping at the digital straws that are represented by Facebook, Twitter, and texting. It is a good bet that we can make informed choices about our use of social mediation technologies. We can become aware of using these technologies and systems in the name of Gemeinschaft.

At the same time, there is a sense of hope that comes out of the data. Earlier in the essay, I noted the use of the mobile phone in the immediate aftermath of the bombing in Oslo. As noted, this was a horrible, but in some ways hopeful insight into our understanding of the potentials of mobile communication. When faced with the awful possibility of a bombing, one of the first reactions of many people was to call their closest friends and family. In an analysis of the geography of these calls, we looked at the billing address of the people initiating and receiving the calls. As one would expect, there were many calls to and from the people located near the bombing. An interesting result, however, was that the calling pattern for the country as a whole showed that people outside Oslo were also calling to their neighbors. People in Drammen were calling other Drammen residents. People in Kongsberg were calling others who live nearby. People in Lillehammer, Bergen, Kristiansand, Rjukan, and Kinsarvik were calling their nearby friends and family. They were calling to share the news and to check on one another. That is, they were enacting society. They were reaching out to one another in a very insecure moment and offering each other a chance to puzzle together the meaning of the imponderable horror of the situation. Interviewing them some months after the event, people remembered whom they had talked to. They remembered the nature of the conversation. In all of its horror, it is at least positive that there was someone who thought to call, and that there was a system available to bring them in contact. The fact that it took place underscores that Gemeinschaft is still there. The impulse to draw on kinship, friendship, and tradition mediated through a digital system is, in itself, a positive thing.

\section{Notes}

1 Jürgen Habermas (1989) has suggested the same in his theory of communicative action and the notion of the "the colonization of the lifeworld" by systems logics.

2 This is similar to what Habermas (1985) would call the lifeworld.

3 I draw on material from Norway since this is the country for which I have the best empirical material; however, I also think it is representative of other Western countries.

4 In a tragic example, analysis of the call traffic patterns in the immediate wake of the July 22, 2011 bombing in Oslo showed that there was an abnormal increase of calls to peoples' strong social ties in the minutes right after the explosion (Sundsøy et al. 2012).

5 Lydia was interviewed in a focus group organized by Telenor in order to understand the use of mobile phones among teens. 


\section{References}

Bailey, B. (1989) From Front Porch to Back Seat: Courtship in Twentieth-Century America, Baltimore, MD: Johns Hopkins.

Beniger, J. R. (1986) The Control Revolution: Technological and Economic Origins of the Information Society, Cambridge, MA: Harvard University Press.

Berger, P. and H. Kellner (1964) Marriage and the construction of reality, Diogenes, 45: $1-25$.

Berger, P. and T. Luckmann (1967) The Social Construction of Reality: A Treatise in the Sociology of Knowledge, New York, NY: Anchor.

Boulding, K. (1955) An application of population analysis to the automobile population of the United States, Kyklos, 8(2): 109-122.

Collins, R. (2004) Interaction Ritual Chains, Princeton, NJ: Princeton University Press.

Durkheim, E. (1938) The Rules of the Sociological Method, New York, NY: The Free Press.

Ehrenreich, B. (2008) Nickel and Dimed: On (not) Getting By in America, New York, NY: Holt Paperbacks.

Elliott, A. and J. Urry (2010) Mobile Lives, Abingdon: Routledge.

Fairtlough, G. H. (1991) Habermas' concept of "Lifeworld," Systems Practice, 4(6): 547-563.

Fischer, C. (1992) America Calling: A Social History of the Telephone to 1940, Berkeley, CA: University of California.

Flink, J. J. (2001) The Automobile Age, Cambridge, MA: MIT Press.

Foster, M. (1983) The automobile and the city, in D. L. Lewis and L. Goldstein (eds.), The Automobile and American Culture, Ann Arbor, MI: University of Michigan Press, pp. 24-36.

Giddens, A. (1991) Modernity and Self-Identity: Self and Society in the Late Modern Age, Cambridge: Polity.

Gordon, E. and A. de Souza e Silva (2011) Net Locality: Why Location Matters in a Networked World, Oxford: Wiley-Blackwell.

Habermas, J. (1985) The Theory of Communicative Action: Volume 2: Lifeworld and System: A Critique of Functionalist Reason, Boston, MA: Beacon Press.

Habermas, J. (1989) The Theory of Communicative Action, Boston, MA: Beacon Press.

Hampton, K. and B. Wellman (2003) Neighboring in Netville: How internet supports community and social capital in a wired suburb, City \& Community, 2(4): 277-311.

Hayden, D. (2003) Building Suburbia: Greenfields and Urban Growth 1820-2000, New York, NY: Vintage.

Ishii, K. (2006) Implications of mobility: The uses of personal communication media in everyday life, Journal of Communication, 56(2): 346-365.

Ito, M. and D. Okabe (2005) Intimate connections: Contextualizing Japanese youth and mobile messaging, in R. Harper, L. Palen, and A. Taylor (eds.), The Inside Text: Social, Cultural and Design Perspectives on SMS, Dordrecht: Springer, pp. 127-145.

Johannesen, S. (1981) Sammendrag av markedsundersøkelser gjennomført for televerket i tiden 1966-1981, Kjeller: Televerkets Forskninginstitutt.

Kumar, K. (1995) From Post-Industrial to Post-Modern Society: New Theories of the Contemporary World, Oxford: Blackwell.

Lee, Dong H. and Duk H. Lee (2012) Increase in telecommunications Expenditure and the migration of consumption online: The case of South Korea, The Information Society, 28(2): 61-82. 
Lenhart, A., R. Ling, S. Campbell, and K. Purcell (2010) Teens and Mobile Phones, Washington, DC: Pew Research Center.

Licoppe, C. (2004) Connected presence: The emergence of a new repertoire for managing social relationships in a changing communications technoscape, Environment and Planning D: Society and Space, 22(1): 135-156.

Ling, R. (2006) Flexible coordination in the Nomos: Stress, emotional maintenance and coordination via the mobile telephone in intact families, in A. Kavoori and N. Arceneaux (eds.), Cultural Dialectics and the Cell Phone: Essays in Social Transformation, New York, NY: Peter Lang, pp. 61-84.

Ling, R. (2008) New Tech, New Ties: How Mobile Communication is Reshaping Social Cohesion, Cambridge, MA: MIT Press.

Ling, R. (2012) Taken for Grantedness: The Embedding of Mobile Communication into Society, Cambridge, MA: MIT Press.

Ling, R., T. Bertel, and P. Sundsøy (2012) The socio-demographics of texting: An analysis of traffic data, New Media \& Society, 14(2): 280-297.

Ling, R., G. Canright, J. Bjelland, K. Engø-Monsen, and P. R. Sundsøy (2012) Small and even smaller circles: The size of mobile phone based core social networks in Scandinavia and South Asia, Journal of Intercultural Research, 41(3): 320-339.

Livingstone, S. (2008) On the mediation of everything: ICA Presidential Address 2008, Journal of Communication, 59(1): 1-18.

Lynd, R. S. and H. M. Lynd (1929) Middletown: A Study in Modern American Culture, New York, NY: Harcourt Brace.

Money, H. A. and R. J. Hobbs (2000) Invasive Species in a Changing World, Washington, DC: Island Press.

Rakow, L. F. (1992) Gender on the Line, Urbana, IL: University of Illinois.

Sheller, M. and J. Urry (2006) The new mobilities paradigm, Environment and Planning A, 38: 207-226.

Shingo, S. and A. P. Dillon (1989) A Study of the Toyota Production System: From an Industrial Engineering Viewpoint, New York, NY: Productivity Press. Also available at: http://books.google.com/books?hl=en\&lr=\&id=RKWU7WE1J7 oC\&oi=fnd\&pg=P $\mathrm{R} 11 \& \mathrm{dq}=\mathrm{A}+$ study + of + the + Toyota + Production + System,+ Shigeo + Shingo\&ots $=$ ni7P $8 \mathrm{y}$ wCiJ\&sig=-ucqHQV61ConthvMX49pc1Sq98k.

Short, J. R. (2006) Alabaster Cities: Urban U.S. Since 1950, Syracuse, NY: Syracuse University Press.

Sorensen, C. E. (1956) My Forty Years With Ford, Detroit, MI: Wayne State University Press.

Sundsøy, P. R., J. Bjelland, G. Canright, K. Engø-Monsen, and R. Ling (2012) The activation of core social networks in the wake of the 22 July Oslo bombing, in 2012 IEEE/ ACM International Conference on Advances in Social Networks Analysis and Mining (ASONAM), pp. 586-590.

Tönnies, F. (1965) Gemeinschaft and Gesellschaft, in T. Parsons, E. Shils, K. D. Naegele, and J. R. Pitts (eds.), Theories of Society, New York, NY: The Free Press, pp. 191-201.

Townsend, A. M. (2000) Life in the real-time city-mobile telephones and urban metabolism, Journal of Urban Technology, 7: 85-104.

Weber, M. (1930) The Protestant Ethic and the Spirit of Capitalism, London: Routledge.

Weller, T. (2012) The information state: An historical perspective on surveillance, in K. Ball, K. Haggerty, and D. Lyon (eds.), Routledge Handbook of Surveillance Studies, New York, NY: Routledge, pp. 57-63. 


\section{Chapter 2}

\section{Walking in the hybrid city \\ From micro-coordination to chance orchestration'}

Robin van den Akker

The moment is born of the everyday and within the everyday.

Henri Lefebvre (2002: 351)

This ... gives a tactical mobility, to be sure, but a mobility that must accept the chance offerings of the moment, and seize on the wing the possibilities that offer themselves at any given moment.

Michel de Certeau (2002: 37)

Mobile information and communication technologies have been traditionally used not only as a means to communicate over long distances but also as a means to coordinate social interaction in space and time. Richard Ling (2004: 69-75), most famously, observed that mobile phones are used to "microcoordinate" social interactions in public spaces by iteratively adjusting, in real time and in midcourse, the most convenient place and the most appropriate time to meet. More recently, the proliferation and popularization of smartphones, equipped with location-aware technologies such as Global Positioning Systems (GPS), has facilitated related, yet different forms of social coordination. Daniel Sutko and Adriana de Souza e Silva (2011: 815-817) observed, for instance, that locative mobile social networks (LMSNs) are primarily used to coordinate sociability in the city (and, secondarily, to explore new places) and that locationbased mobile games (LBMGs) are primarily used to explore new urban spaces (and, secondarily, to coordinate sociability). They write:

One the one hand, LMSNs ... encourage users to communicate and coordinate with other people as the end goal of using the application. As a consequence they might become familiar with places. On the other hand, LBMGs' goals generally include exploring different places in order to play the game. Eventually players might bump into other players and potentially socialize with them.

(Sutko and de Souza e Silva 2011: 817) 
These observations lead us to two interrelated points. It should be noted, first, that both Richard Ling and Leslie Haddon (2001) and Sutko and de Souza e Silva (2011) point to the urbanization of society to contextualize, and explain, the use of, respectively, mobile-based coordination and location-based coordination. The growth (e.g., demographically and geographically) of cities, they argue, shifts the balance to the use of one's position in urban space rather than the use of clock time to coordinate social life. Ling (2004: 73) suggests that in mobile-based coordination one's location ("I am here. Where are you?") determines the reiterative adjustments of the designated place and time to meet, resulting in a softening of time schedules. Sutko and de Souza e Silva (2011: $815)$ go even further to suggest that in location-based coordination "people may increasingly rely on the visualization of space rather than the management of time" resulting in a further trivialization of time schedules.

The second point relates to what Sutko and de Souza e Silva (2011: 816) describe as the tension between "autotelic playfulness" and "teleological navigation" or, put simply, chance encounters and instrumental coordination. "The point we are trying to make here," they write,

is that acts are playful when things are left up to a certain degree of chance.... When acts become too structured or goal-orientated, they lose their playfulness. However, it seems that a chance encounter can become a teleological and hence less playful endeavor when the goal of coordinating with a specific person or a group of persons becomes paramount. Therein lies the potential for a shift from autotelic playfulness to teleological navigation.

(Sutko and de Souza e Silva 2011: 816)

They seem to suggest, here, that location-based media afford either the facilitation of chance encounters or the (micro-)coordination of social or spatial interactions (or something somewhere in between these dichotomous poles).

I do not necessarily disagree with the above points. Yet the following arguments are intended to add another theoretical perspective to the debate on the use of location-based mobile media and to critically intervene in the debate on the tension between (micro-)coordination and the aleatory. I will do so, first, by arguing that users of location-based media often times orchestrate chance. This notion - chance orchestration - is not a substitution for various forms of (micro-) coordination, but should be seen as a supplement to these practices, as users neither coordinate their social and spatial encounters nor simply leave these encounters to chance. Second, I will theorize chance orchestration along the lines of Lefebvre's theory of moments and de Certeau's theory of occasions (Lefebvre 2002: 340-358, 2003: 166-176; de Certeau 2002). Although mobilebased and location-based coordination have indeed resulted in the relative dominance of place over clock time in the coordination of social life, the notion of chance orchestration, thus theorized, demonstrates that the theoretical debate 
about the use of location-based mobile media should remain attentive to its temporal aspects. This is not to say that I will argue for a renewed appreciation of clock time (i.e., chronos) but that the Lefebvrean moment and the Certeaudian occasion point toward the importance of "right points in time" (i.e., kairos) when it comes to the use of location-based mobile media (see, for instance, de Certeau 2002: 43, 83, 98).

The arguments that I will outline in this chapter derive from first, my personal experience with, and observations of, the use of Foursquare; and second, a case study into the use of the location-based service Foursquare by tech-savvy heavy users in their twenties and thirties. For the case study, I have conducted informal, open-ended interviews with ten respondents living in Amsterdam (Netherlands), Rotterdam (Netherlands), and Antwerp (Belgium). All respondents were either studying or working in fields such as ICT, new media or communication. Due to the nature of the interviews and the number of respondents I do not claim to make an empirical analysis based on quantitative research. Instead, the arguments follow from observations and conversations and should be considered as a series of hypotheses to be tested against a larger pool of quantitative data. Ultimately, then, this chapter is an attempt to rethink and reformulate the classic theoretical framework of Lefebvre and de Certeau in the context of the contemporary use of location-based mobile media. It is for this reason that this essay's title is a playful allusion to de Certeau's (2002: 104-118) classic essay on the practice of walking in the city.

Because Foursquare is often seen as a location-based mobile game, the first section of this chapter will look into the application's gaming and competition elements. Foursquare attempts to persuade its users to check in as often as possible and at as many places as possible, and to share these check-ins with as many people as possible by offering points and rewards. The question remains, however, if the users of Foursquare play it by the book? As I argued elsewhere, in a chapter written in collaboration with Linda Kool (2012) and based on the same set of interviews, users often stop seeing Foursquare as a location-based mobile game and sooner or later start using it predominantly as a locative mobile social network (or, to put it in simpler terms, a geosocial network).

In the second section, I argue that the daily use of Foursquare as a geosocial network should be theorized as chance orchestration. I should add, here, that the notion of chance orchestration helps to come to terms with, first, the ways in which Foursquare enables social encounters in the city and, second, the ways in which users become aware of, and act upon, the digital layers with folk knowledge that users of Foursquare are able to create and hang in, or lay over, public spaces. These site-specific layers may result in unexpected spatial encounters or may trigger unexpected spatial practices, as they contain practical tips, critical reviews or poetic observations - anything, really. Finally, I offer some tentative conclusions and some openings for further research. 


\section{Captivating users: gamification, the visibility paradox and social ostracization ${ }^{2}$}

Although Foursquare does not have a closed business model at the time of writing, the company's intentions are clear. By creating profiles based on the spatiotemporal data of its users the company hopes, in the near future, to be able to charge local merchants and international chains a fee to assist them with their location-based marketing. "We have a really advanced way of targeting people," Dennis Crowley (2011), Foursquare’s founder, once said:

Say, you are the kind of person who goes to lots of different pizza places or a lot of different coffee shops. We know about the types of habits that you have. Are you a person that checks-in in the morning? Are you a person that checks-in in the evening? We can start targeting the deals based on your different habits.

To be able to create such refined spatiotemporal profiles Foursquare needs to collect and aggregate an awful lot of data. It therefore needs to persuade its users to keep coming back to the service, time and again. Foursquare needs to convince its users, in other words, to somehow integrate the service into their routines. It needs to "captivate" its users - so that it can change their daily practices (Fogg 2003).

Foursquare's most important captivating technologies are, without a doubt, its competition and gaming elements. Users are, for instance, stimulated to check in as many times as possible at as many locations as possible by rewarding all those check-ins with points that are displayed on a ranking list ("leaderboard"). Users play, as it were, a competition with the people they have befriended on Foursquare. By sending notifications concerning one's ranking ("You're tied with Julie. One more to pull ahead!"), the application urges players to keep checking in. And by rewarding fewer and fewer points when one checks in at locations that have already been visited, the application encourages players to regularly check in at yet undiscovered venues. In an interview in the New York Times (Wortham 2009) Dennis Crowley stated: "The whole point is to encourage people, and reward them for trying new things." Similarly, users are able to earn badges by successfully fulfilling a single task or a quest-like series of tasks. Users can be rewarded, for instance, with a badge for the first, tenth, or fiftieth check-in; a series of visits to museums and fitness centers, bars and restaurants, or train stations and airports; simultaneous check-ins with a group of friends or a check-in under influence (presumably) in the middle of the night and so on and so forth. Several users likened these badges to insignias, suitcase stickers, or passport stamps - whether they must be seen as badges of honor or badges of dishonor is another matter entirely.

The last, and arguably most prestigious, award is the title of mayor, which is "bestowed" on users who have been able to check in at certain locations the most 
within a specific period of time. Of all the gaming elements the race for mayorships seems to be the most captivating. Most users indicated that they, at one point or another, enthusiastically attempted to acquire as many mayorships as possible. "I check in everywhere," one respondent (female, 24, Antwerp) that I interviewed quipped.

I want to beat people. I want to become the mayor. I want to collect mayorships.

One of the interviewees aptly compared collecting mayorships to playing Monopoly. One of the advantages of being the mayor, she explained, is that you place certain locations, as it were, under your "authority," which might result in small benefits such as free goods, product discounts, or better services.

The competition and gaming aspects of Foursquare are part of a broader socio-technological development toward the gamification of everyday life. This development gained momentum following the widespread diffusion of portable ICTs, as these kinds of devices nest themselves in many, if not most of the situations that make up our everyday lives (Zichermann and Cunningham 2011). This is to say that a wide variety, and an increasing number of services attempt to shape our daily practices by means of ludic technologies. Foursquare attempts, in other words, to captivate its users by appealing to humans' innate lust for play (Huizinga 2010). The ultimate goal, the set of practices that reflects Foursquare's desired standard of behavior, would be that its users continuously check in at the locations they are passing by, regularly attempt to check in at locations they had not yet visited, and to share these check-ins with as many friends as possible. Seen from this perspective, then, Foursquare aims to map, as Google would do, the traces that we leave behind while we "surf" through public space (e.g., "who and what, where and when") and attempts to chart like Facebook the social patterns that we create when sharing data with our peers (e.g., "who and what with whom"). The goal is, of course, to get a clearer overview of, and a better insight into, our behavior in public space, so that these data can ultimately be monetized.

The question remains, however, if, and to what extent, users are captivated by all those leaderboards, badges, and mayorships. Do users play along? Are they being played by Foursquare? Or do they not play at all? "I definitely started out as a gamer," one respondent (male, 30, Antwerp) told me.

At first the competitive aspect appealed to me. But after a while I moved on. It's all right. But in the long run you have to find more useful things to do with it.

This changing attitude toward Foursquare's competition and gaming aspects appears to be something that many users share. Most, if not all, do start out as "gamers," but the majority eventually loses interest in just playing games or ends 
up without any interest whatsoever. "When I started using Foursquare," another respondent (male, 25, Antwerp) observed,

I always paid attention to the points that I collected or my ranking on the leaderboard. It used to be a must—but nowadays? Not at all. Not anymore. Less and less, actually.

Of course it might be possible that the experience is not captivating enough to entertain people over a longer period of time. However, this is not how users describe their changing attitudes toward Foursquare's competition and gaming elements. On the one hand, they simply start using Foursquare toward other ends, a process that goes by relatively unnoticed. During one of the interviews, I asked how many mayorships an interviewee possessed. "At least ten," he replied,

mostly of public spaces that are visited by lots of people, such as the bank. Sounds cool, right? Mayor of the bank.

(male, 25, Antwerp)

When he opened the Foursquare application to show off his mayorships, it turned out he still owned not ten but three titles: his house, his parents' house and his workplace. "Apparently I find it increasingly less important," he realized.

I have lost those mayorships unconsciously. Apparently it is something that doesn't concern me any longer.

(It is, by the way, rather common to just hold mayorships of the places where one feels, quite literally, at home). ${ }^{3}$ On the other hand, users consciously stop gaming as soon as they feel it starts to interfere with the other uses they have found for Foursquare. One of the main reasons why users lose interest in competing for points, badges, and titles seems to be the visibility paradox, as I would like to describe it. The more users check in at the places they are passing by - the more visible they are, in other words - the less their friends will be inclined to stop by or come over to those particular places as it seems likely that the former have already moved along, to other locations, and that there will probably not be an opportunity to meet up.

This tension between scoring points and showing your whereabouts-i.e., the signal/noise ratio - is a common concern for users. Even those who describe themselves as "players" are over time becoming more and more reluctant to check in at any occasion. One respondent (female, 24, Antwerp) explained:

When I first started using Foursquare I checked in everywhere. Even when I just passed by a place and I didn't have to be there. Just to get the points. 
Over time, however, I stopped doing that. I still want to be among the top flight of the leaderboard, but it's less important. Now I just check in at the places where I am about to stay for a while.

Most users that I have spoken to made similar observations. There seems be some kind of consensus, a set of shared norms, concerning the most appropriate moments and places to check in and share one's location. People who transgress these norms - the so-called "hit whores," as one user put it —risk being ignored at best and ostracized at worst. ${ }^{4}$ Interestingly, users have developed various strategies to avoid being seen as obtrusive, ranging from a self-imposed "check-in cap" to clandestine "off the grid" check-ins.

\section{Using Foursquare: digital metis, software and network orchestration}

These conscious and unconscious decisions to check in one moment and not the next while leaving the game for what it is, and to share only some, and not all, locations suggest that the tech-savvy heavy users in their twenties and thirties that I interviewed succeeded in "domesticating" the application (see, for instance, Silverstone and Haddon 1996). ${ }^{5}$ These users also indicated that they predominantly use Foursquare to become aware of interesting places, practices or people in the nearby environment; and that they become aware of these places to visit or people to meet by either proactively scanning urban space aided by the application or by reacting to its push-notifications. Users of Foursquare, then, continuously run into unexpected opportunities, presented by circumstances, such as a spontaneous meeting with someone who checks in at a nearby location or an unexpected spatial practice triggered by a nearby layer with folk knowledge. ${ }^{6}$ This particular use of Foursquare - and other geosocial networks - can be conceptualized as chance orchestration.

It should be noted that chance orchestration seems to be a rather oxymoronic notion. In computer handbooks, after all, orchestration refers to the creation of assemblages of hardware, software, and human operators to deliver very specific services, to eliminate chance. Yet this oxymoronic notion starts to make a lot more sense when theorized along the lines of Henri Lefebvre's "theory of moments"7 (2002: 340-358, 2003: 166-176) and Michel de Certeau's "theory of occasions" (2002), which the latter used, perhaps most famously, in his essay on walking in the city.

A moment, claims Lefebvre (2002: 343), "is not just any old instant." In a moment one perceives the glimpse of a possibility, an opening in the cracks of the everyday, while being in the midst of the city's bustle, suddenly and unexpectedly, by chance $(2002: 343,348)$. Although "chance" seems to be a rather straightforward notion, it is as hard to come up with a definition, as it is difficult to list all of its uses, meanings, and connotations (as it is for so many of the seemingly self-evident notions that come under scrutiny when studying everyday 
life). For the notion of chance and its related categories have, as De Mul (2006: 54) shows, a rather confused and confusing history, which can be traced back to Aristotle's writings and its various translations, interpretations, and adaptations in Latin and, ultimately, the various vulgar European languages. Yet there are three fundamental categories, which are also referred to by Lefebvre in relation to the theory of moments. He writes, for instance, that the "circumstantial content" of the moment derives from "something happening close by, something contingent and accidental" (2002: 346, my italics). Let me briefly explain these fundamental categories (in reverse order).

First of all, chance can be conceived of as the accidental, which refers to the circumstantial, non-essential attributes of something, anything (De Mul 2006: 55). Whereas a man's essential attributes are that he is born, and ultimately dies, someone's gender and race, habitus and habits, career path, and life trajectory are accidental. Similarly, and more related to this essay's topic, the essential attributes of geosocial networks is location-awareness, yet whether one uses Gowalla or Foursquare on a Samsung Galaxy or an iPhone is accidental.

Second of all, and more interestingly, chance can be conceptualized as the contingent, which refers to anything that is possible, and to anything that is neither impossible nor necessary. The contingent is that which could possibly be, but has no facticity; or that which could have possibly not been, but does have facticity. During our everyday dealings with the world and with others we, in fact, constantly navigate such contingencies as we are presented with countless possibilities of which only one at a time can be actualized. Once a certain possibility is actualized, moreover, some (chains of) possibilities are closed off and others open up (De Mul 2006: 56-57). When you decide to accept a friend request of a so-called "familiar stranger" (Milgram 2010 [1977]), for instance, you set in motion a chain of events that might lead to an enjoyable conversation or an amicable relationship-though you befriended the familiar stranger as the possibility presented itself, it could have been otherwise.

Lastly, chance can be conceived of as fate, which refers to all those things that are happening to us while we were busy making other plans. Whereas our contingent dealings with the world and others presuppose a set of possibilities, presented by circumstances, of which some are chosen to be actualized and others are chosen to be discarded, fate just comes by uninvited, either as a wanted or an unwanted guest, independent of our practices. Chance as fate can, in other words, be used to describe both unforeseen events and unintended consequences (De Mul 2006: 60-61). In the context of the use of Foursquare, the first category might relate to a sudden "notification" of a site-specific layer of folk knowledge that one of the users you befriended "hung" in the space that you are traversing, triggering an unexpected spatial practice or spatial encounter. The second category relates to anything that happens after a certain practice, which could not happen without that particular practice, but has never been the goal of that practice. Aristotle gives us 
the example of the man who goes to the market to buy sustenance, but runs into one of his debtors (ibid.). Yet one could also think of someone who travels home from work and is notified by Foursquare that his friends are having a coffee just around the corner so that he can stop by.

These examples indicate that applications like Foursquare alter all of the above-described dimensions of chance. When users choose to use Foursquare rather than (the by now defunct) Gowalla this seems to be an accidental choice from among the available geosocial networks. Yet Foursquare's position as a so-called "market leader," which promises a critical mass of users, makes this choice less accidental. The choice for Foursquare also means a choice for an interface that selects certain contingent, unforeseen, or unintended occasions presented by circumstances (and excludes others). Let me explain.

The moment, Lefebvre (2002: 351) argues, is born of the everyday and in the everyday. As soon as a moment is perceived, "a choice ... singles it out and separates it" from those transitory instants that ordinarily make up everyday life (ibid.: 344). This choice reorganizes the here-and-now as the moment is "closed off by constitutive decisions. Anything which cannot be included is chased away" (ibid.: 353). However, the attempt to realize its possibilities is of limited duration; the moment cannot last. It instantaneously emerges from everyday life, temporarily gains momentum, until it definitely exhausts itself (ibid.: 345). This tragic dimension of the moment does not make the attempt futile- on the contrary. It creates a "history" and it is "memorized" and future moments are recognized on the basis of its legacy (ibid.: 345-346).

Readers who are familiar with the writings of de Certeau might have already discovered, between the lines and in an embryonic stage, most of the central arguments of The Practice of Everyday Life (2002). Although he never cares to acknowledge his debt to Lefebvre, ${ }^{8}$ it can be argued that the linchpin that holds together the elements that support most of the Certeaudian edifice-i.e., the famous distinction between strategies and tactics - is the theory of moments disguised as a theory of occasions.

De Certeau's analysis of the practice of walking in the city is the paradigmatic case in point. On the one hand, the argument goes, spatial strategists organize an "ensemble of possibilities ... and interdictions," related to the dominant ways of "conceiving and constructing space," by means of which everyday practices are assigned a proper place, and a proper time. On the other hand, walkers manipulate this regime of set practices by tactically taking advantage of occasions to seize opportunities presented by circumstances (2002: xix, 43, 83, 98). Tactical practices, then, "use, manipulate or divert" by taking advantage of occasions — "right points in time" (2002: 29-30) —offered by the urban context at hand.

To be sure, de Certeau deliberately uses a temporal notion to describe the tactical nature of spatial practices. Given the fact that one must "play on and with a terrain" that is strategically produced, taking advantage of occasions requires a certain "tactical mobility," which "must accept the chance offerings 
of the moment, and seize on the wing the possibilities that offer themselves" (ibid.: 37).

Occasions, importantly, consist of "heterogeneous elements" to be combined in an "intellectual synthesis" that "takes the form ... of the decision itself; the act and the manner in which the opportunity is seized" (ibid.: xix). Constitutive decisions include some elements of the given circumstances, and exclude others, by processing, in a fraction of time, all relevant data concerning the occasion, the opportunity, and the possible outcomes. These constitutive decisions are mediated - and this is his most valuable contribution to Lefebvre's insights - by a form of knowledge ("metis") that is founded on experience, practical wisdom, and making do, and is "composed of many moments" (ibid.: 82). He rather elegantly describes metis as a "memory" or as the smallest of volumes of an encyclopedia, which nevertheless holds both a "treasure of past experiences" and an "inventory" of future opportunities (ibid.: 82-83).

What do the combined insights of Lefebvre and de Certeau tell us about the practice of walking in the hybrid city? What is it that we do when we use Foursquare? It could be argued that users of Foursquare are outsourcing some-not all! - aspects of their metis to their technological extensions. Although the application seems to enable an infinite number of chance encounters and random discoveries, users do not become aware of all the things that are happening in their surroundings and they do not become aware of all user-created layers in their environment. Foursquare does not turn the screens of our smartphones into boundless windows of opportunity. For the constitutive decisions that include some elements of the given circumstances and exclude others are mediated by one's digital metis. The chance offerings of the moment are orchestrated by means of the use of Foursquare.

We can discern two forms of chance orchestration: network orchestration and software orchestration. Network orchestration can be described as the adaptation of one's social network to one's needs, desires, and wishes. By befriending (i.e., including) and unfriending (i.e., excluding), users constantly groom the quantitative and qualitative aspects of their social networks. Geosocial networks enable users, in other words, to become aware of a more or less well-defined set of social and creative practices happening in their (not-so-)immediate surroundings. Foursquare facilitates, in other words, the creation of an archive that provides an insight into the history of the spatial practices of one's social peers and a map that provides an oversight of their current spatial practices. Users are alarmed by a push-notification, for instance, when a friend checks in at a certain location ("Julie just checked in at..."), which enables users to meet up when they accidently happen to be nearby. "A buddy of mine," one respondent (male, 25, Antwerp) for instance told me,

was in another bar than me, and he checked in. I hadn't seen him in a while, so I sent him a message: "Long time no see. Stay there. I'm coming over." That's great! Especially, here, in the city, where everything is in walking distance. 
Similarly, Foursquare sends push-notifications when users coincidentally pass by a place or spatial practice that has been (previously tagged and) recommended by one of the users they have befriended ("since you're so close, why don't you try Julie's tip"). And, finally, users can subscribe to "to-do"-lists, created by other commoners, that send notifications when one by chance stumbles upon a point of interest.

Software orchestration can be described as the willingness of users to rely on the algorithms that filter the heterogeneous elements that make up chaotic urban environments. By using geosocial networks such as Foursquare, users constantly update databases containing the digital traces of their spatial practices - consciously and unconsciously. These databases contain our personal and collective track records (i.e., who, what, where and when, with whom) or, put differently, the accumulated memories of previous occasions and moments. They are, in other words, in many ways similar to those "encyclopedias" that hold both a treasure of past experiences and an inventory of future opportunities. This should not come as a surprise. In our contemporary culture, as many a philosopher of technology has argued, the database ontology is underlying most, if not all, aspects of our everyday lives (Manovich 2001; De Mul 2009). As data can be (re)combined at will, these dynamic inventories theoretically consist of an entirely random and sheer infinite list of opportunities and possibilities. Yet this is not the case.

Whether kept in encyclopedic form or as an actual database, the accumulated memories of previous occasions and moments (in)form both the capacity to recognize new ones offered by circumstances and the attempts to tactically take advantage of the possibilities and opportunities thus perceived. Foursquare's explorer function, for instance, enables users to perceive a series of recommended places and spatial practices, located within a range of 250 meters to ten kilometers. "This an ideal 'tool'," one respondent (male, 25, Antwerp) observed.

Before I go somewhere, I look up what is popular and what is not popular. And the "friend" aspects also plays a role.... Once I noticed that there were a lot of restaurants around me with things on offer. So I searched for "food" and stuff, and then you can see tips, how many people have been there and how many friends, the popularity of the place.... It's like a GaultMillau or a Michelin Guide for ordinary people.... So I saw that somebody I know, the girlfriend of a colleague, suggested a certain burger, and I tried it.... It's weird... Normally, when you work here and the train station is there, you just travel that distance and you do not travel another distance. We are trapped in our routines. With this you say: "yeah, I am going to walk into that other direction. A lot of people have checked in there; there are a lot of good restaurants and nice shops."

Generally, such personal recommendations are based on the aggregated spatiotemporal data of individual users, befriended users and all other users. Foursquare's algorithms select, in other words, the places that someone has already 
visited ("you've been here 9 times"), all similar places ("you've been to 2 related places"), the places that have been visited by his or her friends (" 1 friend has been here"), and the places that will probably be of interest to a user as other people with similar profiles have already checked in there ("People who go to this place, tend to go to that place"). Additionally, users can look up which places proved to be popular over time ("recommended") and which places are being busily frequented at the moment ("trending").

We can conclude, therefore, by observing that the orchestration of chance by means of network orchestration and software orchestration entails that users neither accept the aleatory character of city life nor circumvent it by coordinating spatial and social interaction. In the resulting situation, paradoxically, the number of possible chance encounters go up (as they are orchestrated), while the odds to walk into something or someone interesting by chance go down.

\section{Conclusions and openings}

In this chapter I hope to have shown that, for the users of Foursquare- or rather for the tech-savvy users in their twenties and thirties that I interviewed-the capacity to orchestrate chance encounters (e.g., in the social and spatial sense of the word) often outweighs the possibility to play games. The analysis of the locative game Foursquare showed that its competition and gaming elements might persuade users to adopt the application, but sooner or later its captivating aspects - scoring points, winning rewards, showing off, competing with others, etc.- - lose most of their persuasive power. The reasons may vary from the discovery of other uses for the application to the visibility paradox to the fear of being ostracized from, or ignored by, one's social circle.

The analysis of the geosocial network Foursquare, meanwhile, showed that the notion of chance orchestration is more helpful to come to terms with the daily use of the application, as users neither coordinate their social and spatial encounters nor simply leave these encounters to chance. The choices that set certain occasions or moments apart and the decisions to include some elements of the urban environment and to chase away anything that cannot be included, are, in fact, mediated by a form of digital metis. Foursquare thus enables users to upgrade - to cyborg-like proportions - their capacity for kairos, which increases their ability to take advantage of the moments or occasions that are born of the everyday and within the everyday.

There are still many questions unanswered about the nature of chance orchestration and its consequences for the practice of walking in the hybrid city. One particularly interesting line of inquiry consists of the composition of the network of befriended users that people maintain by means of Foursquare. Do these networks consist of previously existing friends or people who were befriended, and could only be befriended, by means of Foursquare? Interestingly, the social circles that are maintained by the Foursquare users that I interviewed, consist of those who are close to them in the social sense of the word - their "close friends" 
with whom they maintain "strong ties" - and/or in the geographical sense of the word - their "fellow travelers" with whom they, previously, would have had very weak ties or no ties at all. Foursquare is, in these particular cases, both used as a means to strengthen existing relationships and often used as a way to forge new relationships, both as an instrument for bonding within and bridging between urban communities. Obviously this hypothesis should be tested in a quantitative research that is much more elaborate than my own qualitative study.

The outcome of such a study, however, could have interesting ramifications for the tension between "relevance" and "serendipity," as Eli Pariser (2011) put it. He argues that our online practices increasingly take place within a so-called "filter bubble" as a result of the proliferation of predictive algorithms and the homogenization of online social networks. Such filter bubbles, then, ultimately cater to already established preferences, interests, and desires and, as a result, we almost never stumble upon things that do not fit our way of life and our particular perspective on the world. Does the use of Foursquare, then, result in what could be described as an urban filter bubble (see also Gordon and de Souza e Silva 2011: 88)? Or, put differently, could chance orchestration lead to truly serendipitous social and spatial encounters (see also de Souza e Silva and Frith 2012: 167-173)? Does Foursquare allow for the chance discovery of people, places, and practices one could have never guessed finding interesting as they do not fit with one's preexisting patterns of social and spatial preference? At first sight the answer to all these questions seems to be no. As I have argued, users of Foursquare rely on software orchestration and network orchestration to orchestrate chance while walking in the hybrid city. These orchestrated chance encounters thus seem to take place within an increasingly refined urban filter bubble that feeds back to the user what he or she feeds to the algorithms of and the networks of Foursquare. Yet the solidity or porosity of any filter bubble ultimately depends, among other things, on the composition of one's network of befriended users (and the networks of their friends). The case for existence or inexistence of an urban filter bubble therefore still needs to be made.

\section{Notes}

1 I would like to thank the two anonymous reviewers for their insightful suggestions for improvement.

2 The arguments in this section have been developed in collaboration with Linda Kool at the Department of Information Societies of The Netherlands Institute of Applied Scientific Research (see van den Akker and Kool 2012).

3 One respondent (male, 21, Amsterdam) told me, for instance, that he for weeks "battled" for the mayorship of a particular bridge next to his house that he daily crossed because he felt it was "his" bridge - and crossing it "felt" like homecoming.

4 Similarly, following de Souza e Silva and Frith (2012: 162-167), users may also selectively check in because, in doing so, they are able to "present" themselves through location in different ways, and construct their personal identities spatially.

5 The users domesticated Foursquare in the sense that they adapted and moved beyond the application's default settings and desired standards of behavior and found other 
ways of using the app, better suited to their everyday lives. As a result of this process of (what Silverstone calls) appropriation the users have also changed. They developed new routines, as well as new ways of perceiving, conceiving, and being in urban space. Consider, for instance, what happens when you take a young dog into your house. The dog will be domesticated over time, yet your household will also be changed.

6 It should be noted here, that the relative emphasis on chance orchestration over gaming that I found in my research is not so much a tactical appropriation or, even, subversion of the strategic persuasive intentions of the developers of Foursquare. It simply seems that the users that I interviewed find more use value in chance orchestration than in gaming. It may very well be, as one of the anonymous reviewers rightly pointed out, that the "domesticated" usage of Foursquare may be just as useful for tracking spatiotemporal behavior and might as well contribute to the monetization of the application. As I see it, chance orchestration appears to be a novel way of tactically appropriating the chance character of the practice of walking in the city.

7 The Moment, to be sure, does not pertain to such dramatic instants of revolutionary fervor as, say, the 1848 Commune or May 1968 - quite the contrary (Lefebvre 2002: 340-358, 2003: 166-176). Most of the time, if not always, Lefebvre uses the notion for a "more modest," but "broader" phenomenon to be situated within the "history of the individual in his everyday life" (Lefebvre 2002: 344).

8 Only once does de Certeau refer to Lefebvre. In a footnote to his introduction he acknowledges that Lefebvre's writings have had a mayor influence on his work on everyday life. He never indicates which parts of Lefebvre's oeuvre he has used and to what extent Lefebvre influenced him. However, the footnote is strategically placed after the word "anti-discipline," which he uses to summarize his entire research project. The correct reference should have been to Lefebvre's theory of moments and not, as is commonly assumed, to Lefebvre's The Production of Space (1991).

\section{References}

Crowley, D. (2011) Foursquare's growth, merchant platform, revenue, Bloomberg, available at: www.bloomberg.com/video/73366536-foursquare-s-growth-merchantplatform-revenue.html.

de Certeau, M. (2002) The Practice of Everyday Life, Berkeley, CA: University of California Press.

De Mul, J. (2006) De Domesticatie van het noodlot. De wedergeboorte van de tragedie uit de geest van de technologie, Kampen: Klement.

De Mul, J. (2009) Database architecture: Anthropological reflections on the art of the possible, Journal of Asian Arts \& Aesthetics, 3: 1-9.

de Souza e Silva, A. and J. Frith (2012) Mobile Interfaces in Public Spaces. Locational Privacy, Control and Urban Sociability, New York, NY/Abingdon: Routledge.

Fogg, B. J. (2003) Persuasive Technology: Using Computers to Change What We Think and Do, San Francisco, CA: Morgan Kaufmann Publishers.

Gordon, E. and A. de Souza e Silva (2011) Net Locality: Why Location Matters in a Networked World, Oxford: Wiley-Blackwell.

Huizinga, J. (2010) Homo ludens: proeve eener bepaling van het spel-element der culturen, Amsterdam: Amsterdam University Press.

Lefebvre, H. (1991) The Production of Space, Oxford: Blackwell University Press.

Lefebvre, H. (2002) The Critique of Everyday Life: Foundations for a Sociology of Everyday Life, London/New York, NY: Verso.

Lefebvre, H. (2003) Henri Lefebvre: Key Writings, London/New York, NY: Continuum. 
Ling, R. (2004) The Mobile Connection: The Cell Phone's Impact on Society, San Francisco, CA: Morgan Kaufmann Publishers.

Ling, R. and L. Haddon (2001) Mobile telephony, mobility and the coordination of everyday life, paper presented at the conference "Machines that Become Us," Rutgers University, New Brunswick, New Jersey, April 18-19, available at: http://itu. $\mathrm{dk} / \sim$ christie/mobilspeciale/litteratur/Ling,\%20Rich\%20-\%20Mobile\%20Telephony\%20 Mobility\%20and\%20the\%20Coordination\%20.pdf.

Manovich, L. (2001) Language of New Media, Cambridge, MA: MIT Press.

Milgram, S. (2010 [1977]) The Individual in a Social World: Essays and Experiments (third expanded edition), Thomas Blass (ed.). London: Pinter \& Martin.

Pariser, E. (2011) The Filter Bubble: What the Internet is Hiding From You, New York: Penguin Press.

Silverstone, R. and L. Haddon (1996) Design and the domestication of information and communication technologies: Technical change and everyday life, in R. Silverstone and R. Mansell (eds.), Communication by Design: The Politics of Information and Communication Technologies, Oxford: Oxford University Press.

Sutko, D. M. and A. de Souza e Silva (2011) Location-aware mobile media and urban sociability, New Media \& Society, 13(5): 807-823.

Van den Akker, R. and L. Kool (2012) Buiten de gebaande paden of binnen het eigen straatje. Geosociale netwerken en de orkestratie van toeval, in C. Van 't Hof, J. Timmer, and R. van Est (eds.), Voorgeprogrammeerd. Hoe het internet ons leven leidt, The Hague: Boom.

Wortham, J. (2009) Foursquare seeks to turn nightlife into a game, The New York Times, March 13, available at: http://bits.blogs.nytimes.com/2009/03/13/ foursquare-seeks-toturn-nightlife-into-a-game/.

Zichermann, G. and C. Cunningham (2011) Gamification by Design: Implementing Game Mechanics in Web and Mobile Apps, Sebastopol, CA: O'Reilly Media. 


\title{
Direct video observation of the uses of smartphones on the move
}

\author{
Reconceptualizing mobile \\ multi-activity
}

\section{Christian Licoppe and Julien Figeac}

A recent video of a woman falling into water in a mall while texting on the phone went viral on YouTube. ${ }^{1}$ It was used as a resource by mobile communication theorists such as Howard Rheingold to support their claim that the management of attention (the way it is done, our awareness of it, etc.) is a big issue in today's world of multiple screens and mobile terminals (Rheingold 2012). This representation of how mobile phones disrupt users' attention and threaten the propriety of conduct concurs with a line of dystopian critique originating in the early days of the mobile phone, which gave rise to significant media coverage about the potential use of mobile phones in public settings varying from restaurants to the classroom and funerals. ${ }^{2}$ These articles criticized not only the way mobile users might become involved in mobile communication in urban settings, but also their alleged inability to cope with the demands of sociality in public situations due to this involvement. Some recent, observation-based research on the uses of portable devices in public places suggests a more nuanced picture (Hampton et al. 2010): while a proportion of mobile device users may display a deficit of attention (both with respect to "eyes-up" behavior, and in terms of reaction to external stimuli), a more important proportion still display some degree of attunement to their environment (Hampton et al. 2010). Unfortunately, such empirical research does not give access to the way users manage their attention, locally, on a moment-by-moment basis, as situations unfold, and in a way that is largely non-reflexive. So neither traditional methods such as interviews and surveys, nor the big data quantitative approach based on the study of various use-related logs, provide appropriate evidence.

We argue that researchers unavoidably need detailed video recordings of users' conduct in everyday situations to make sense of the situated practices involved in the management of attention. In this chapter, our aim is two-fold. First, we wish to describe a portable method for video recording that uses mobile phones and smartphones on the move. Second, we will use this method in an empirical study of the use of smartphones in transport situations to gain new insights into what the joint management of mobile communication and mobility, and more generally multi-activity, is about. The development of this method is therefore important to understand multi-activity because it allows us to describe 
the practical accomplishment of this phenomenon and analyzes how mobile technology-based activities renew the patterning of social activities, such as daily commuting and urban mobilities.

Video recordings made with portable devices enable the development of promising methods because they may "follow" the user and record his visual experience. This constitutes a powerful way to obtain naturally occurring data regarding how smartphone users manage mobile applications in a manner that is sensitive to the context of use and to the other activities the user is involved in at the time. This method is useful because it improves our understanding of multiactivity. Research on time use distinguishes several forms of multi-tasking, such as "simultaneous multi-tasking" or "consecutive multi-tasking" (Kenyon 2010). We use here the notion of multi-activity to account for the practical accomplishment of multiple streams of activity with different temporal organizations, that alternates phases of engagement and disengagement in embodied performances, for example, while talking and driving (Laurier 2000; Haddington and Rauniomaa 2011). Previous research has focused on video recording drivers' and passengers' behavior in cars and using multimodal interaction analysis by putting cameras in the automobile (Laurier et al. 2008; Mondada 2012). Some of this work has explicitly looked at the way car users collaboratively manage phonerelated events (Esbjornsson et al. 2007; Haddington and Rauniomaa 2011) or GPS-based information (Brown and Laurier 2012). To complete these studies, we developed a portable recording device in order to describe more precisely how drivers manage handheld devices and perform multiple activities while driving. More generally, these video protocols defined to analyze interactional arrangements between drivers and passengers are not adapted to other mobile settings, such as the study of daily commuting in public transport. So, one has to turn to more flexible mobile recording devices. Cameras following users along their mobility paths have been used in visual anthropology, where the analyst may visually "shadow" and record the person's engagement in places and orally record his comments to produce a self-centered visual tour (Pink 2007). If such set-ups are useful in understanding how mobile persons are engaged in places, they usually do not provide good empirical access to the persons' use of digital technology as they move. For that purpose, it is better to have the user wear or carry the recording device attached to his or her body in a mobile cyborg assemblage, such as in Steve Mann's experiments in "wearable computing” (Mann 2012).

There again, different recording methods provide different empirical data and support different types of analytical claims. When the camera is worn only on the torso of the user, it aims to provide a view of the context and a general sense of the user's body, without much access to what he or she might actually be gazing at or attending to. When the video recording is produced from the user's head, stronger claims are usually made about capturing, more or less accurately, the user's visual experience "in action." Oculometric methods are most precise because they record eye movements and fixation patterns (Yilmaz et al. 2006). 
They have also been applied to the use of mobile phones (Drewes et al. 2007), but oculometric devices are not easily portable and are cumbersome to use outside the laboratory. Another approach is to ask the user to wear a single camera on his head, either on the subject's forehead (Omodei et al. 1997) or on specially fitted glasses, sometimes described in literature as "video glasses," "subcams," or "camera glasses."

Camera glasses have been claimed to enable the analyst to record the evolution of the subject's visual field in a situation and capture something of his subjective perspective (Lahlou 2011). They have been used by several groups to study mobile phone uses in natural settings (Mark et al. 2001; Relieu 2002; Figeac 2012), either alone or coupled to log data analysis (Zouinar et al. 2004; Oh and Lee 2005). But when camera glasses alone are used, they rarely provide a resolution of the mobile phone screen good enough to grasp the finer details of mobile use. It is therefore useful to complement this context-aware device with a screen-capture recording, for the latter will provide precise and independent access to the user's activity on the mobile terminal (Relieu 2009; Roto et al. 2004). Since we are interested in fine-grained data about how users manage the joint demands of mobile communication and mobility, we have decided to combine here the recording produced by camera glasses with the mobile screen video capture apparatus.

In this chapter we will show precisely how the contextual data provided by our dual recording system can document the analysis of the manner in which sequential aspects orient users toward the ongoing situation. This study of the temporal organization of activities helps to account for how users distribute their attention between mobility and mobile communication concerns, particularly in terms of how users are absorbed by the mobile phone screens. This sort of absorption is an additional step in the historical framework of the current social changes of attentional processes in our contemporary technological culture (Crary 1999). It refers primarily to the capacity of users to switch quickly between phases of deep attention and hyper attention (Hayles 2007). These switches between cognitive modes are sometimes interpreted as a distraction and a problem to be solved. From our point of view, this is a competence of the users that we need to better understand in order to design and implement new choreographies of attention, for example by building sustainable models for knowledge production (Gordon and Bogen 2009). By describing the temporal organization of gaze switches, our video recordings allow us to do just that as we analyze this dimension of absorption and attentional processes.

These data equally help to identify some local and situation-specific user concerns, while carefully avoiding hyperbolic claims that we would gain access to the user's subjective experience, or that what the analyst can "see" through the camera glasses video is actually what the user "sees." We will show that such a recording method makes it possible to observe the temporal organization of gaze switches, i.e., the way the mobile user's gaze moves toward or away from the mobile phone. By combining the data from the camera glasses and the screencapture recordings, gaze switches can be documented and their occurrence 
linked to that of various events happening in the mobile interface or the larger situation. Such data can therefore inform us on how users orient to contingencies relevant to mobile communication as well as to other relevant streams of activity (such as mobility) on a "moment-by-moment" basis.

To show the analytical usefulness of such an approach, we have done an empirical study on the uses of smartphones in transport situations. We analyze in detail here one particular case in which we observe a driver trying to juggle the constantly evolving demands of mobile communication and traffic. This enables us to provide an empirically grounded definition of multi-activity as a particular way of being attuned to events in one stream of activity as opportunities to switch one's attention and involvement toward another relevant stream of activity, i.e., to what we call here their sequential implicativeness. This highlights the importance of the way in which the accomplishment of a given activity in a specially designed environment "affords" events with possible sequential implicativeness (that is, conducive to gaze switching). It will allow us to develop ideas about designing specifically for multi-activity settings, and more particularly, designing mobile communication application interfaces better fitted to the specific demands of multi-activity settings, as with mobile communication and transportation.

\section{Methodology}

Analyzing the uses of mobile communication terminals in everyday settings requires synchronized empirical data regarding both the use of the mobile device and the off-screen involvements and activities of the users. What we have done is to combine smartphone screen captures with the recordings of the contexts of use produced by user-worn camera glasses. To capture the screen-based activity, we have used a feature of Android-based smartphones that allows an audio and video connection. We have therefore coupled the smartphone with a light portable A/V recorder (Figure 3.1), a method initially developed to study mobile video telephony (Morel and Licoppe 2011). When the system is on, it provides a recording of the changing mobile phone screen as the user performs various actions upon it, in the form of a video file.

While such a portable set-up may provide rich detailed data on the "natural" uses of smartphones on the move, it provides very little information on the actual contexts of use. We have asked users to wear camera glasses to obtain a video recording of the users' changing environments that can be synchronized to the mobile device screen-capture data. Camera glasses have been used by various authors to gain a "subjective view" of actors performing various activities (Lahlou 2011). What was interesting to us was that the whole recording apparatus was portable and could be used to obtain data on the uses of smartphones in mobility settings.

However, the temptation for the analyst is to look at what the camera glasses record as the actor's visual perception of his environment. Yet what you get is 


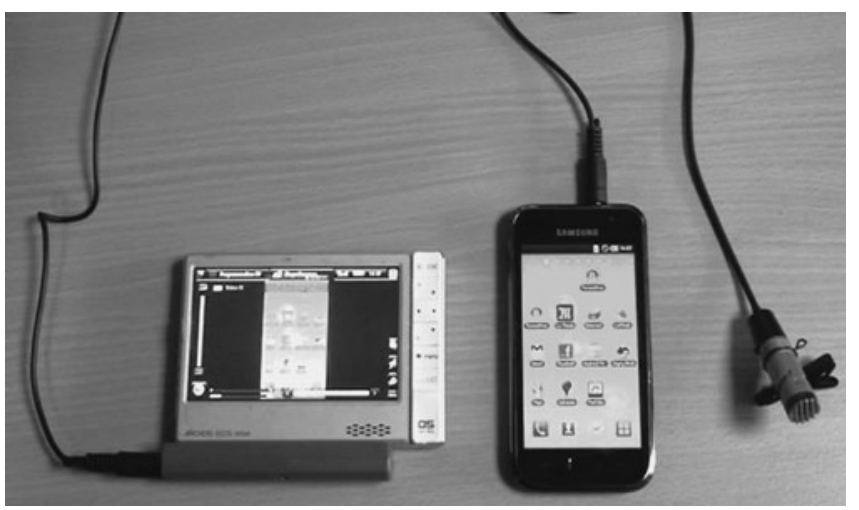

Figure 3.I A simple portable set-up to record the audio-video flux on Androidbased smartphones (@ Christian Licoppe and Julien Figeac).

not what he sees. Because of various constraints, it is often a rather crude approximation of human perception. The recording field is only about 40 degrees wide for standard commercial camera glasses, which is much narrower than the human field of vision. So the camera glasses data will not show what is available in the peripheral visual field of the human subjects. A consequence of this is that when different subjects are recording with camera glasses at the moment they are sitting and looking at their smartphones, the recording may feature the phone screen only partially (Figure 3.2) or not at all (Figure 3.3) in the video recording. In spite of all this, camera glasses are easy enough to use and wear to remain an attractive method to gather naturalistic observations on the move, provided we can somehow circumvent some of these limitations.

Although what you get is not what the subject "sees" and the data has to be treated with care in the analysis, camera glasses may still be used, albeit with a degree of caution. First, the analyst is helped by the small size of the smartphone screen. To look at the smartphone, the user needs to orient his gaze in a rather precise direction, which can be reconstructed in the analysis and confirmed by the screen-capture data (which shows what action he is currently involved in on the mobile interface), even if the smartphone is not actually visible on the video data. However, when the user looks away from the phone, it may become more difficult to infer what he might be looking at from the video glasses only. Hence, what camera glasses applied to the uses of smartphones on the move make visible are gaze switches, that is, when the users look toward or away from their smartphones. Such gaze switches are interpretable as switches between attending to the smartphone (which we may further document from the screen capture) and attending to other meaningful domains in the environment. Such data therefore provide a rich source of information on the way smartphone users may manage multiple involvements in actual public settings. 


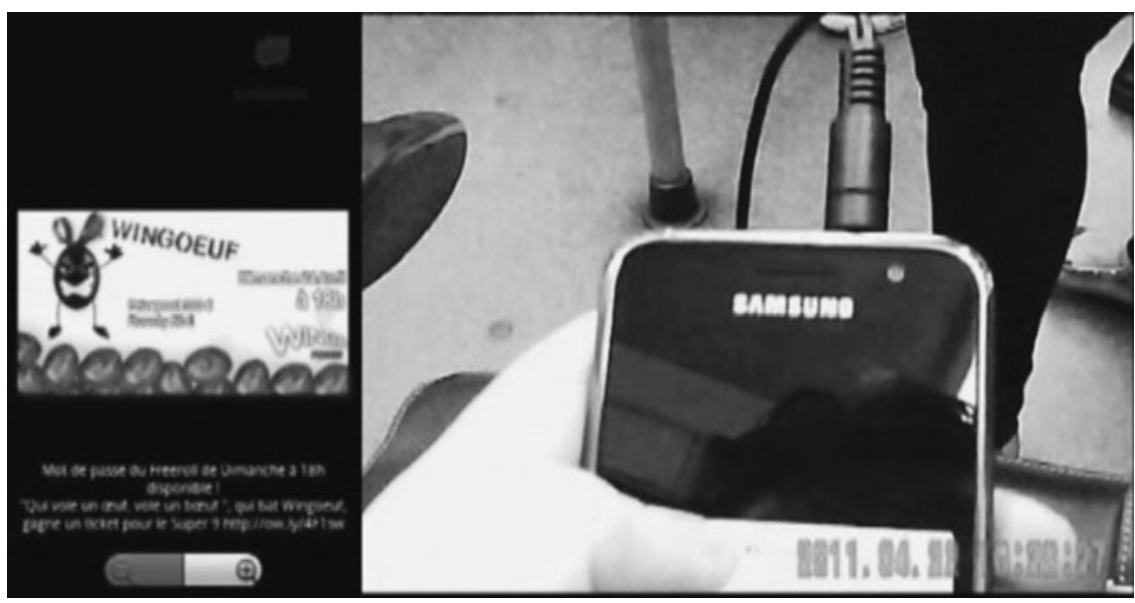

Figures 3.2

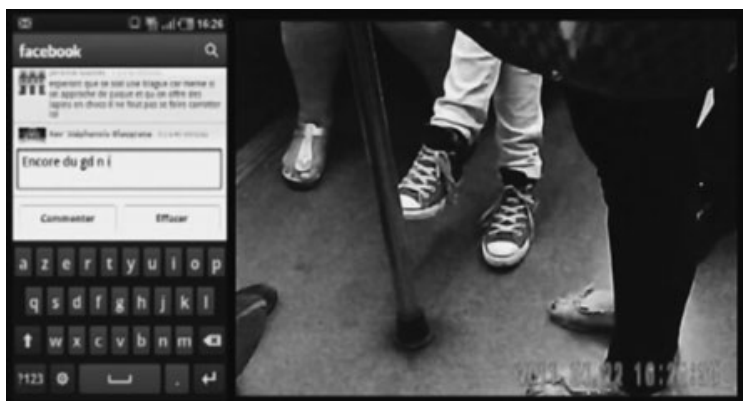

Figures 3.3

Figures 3.2 and 3.3 In all images the screen capture appears on the left, and the camera glasses recording on the right (both have been synchronized). In both images the user is sitting and using the smartphone in his lap, which is partly (3.2) or not at all (3.3) visible (@ Christian Licoppe and Julien Figeac).

We will focus here on the joint management by users on the move of mobile social networking applications and everyday mobilities, especially automobilities, in order to demonstrate the potential of our research method. We will gather empirical data on gaze switches toward and away from the smartphone, and we will analyze their temporal patterning. Our research question will then be "Why this gaze switch now?" (a) to show that there are some recognizable and understandable patterns in the temporal placement of gaze switches; (b) to show that such patterns inform us on the way the temporal organization of the different relevant activities are used as resources in their "simultaneous" management; and 
(c) to draw some implications from this for the design of technologies and applications that might be particularly fitted to multi-activity settings.

\section{Fieldwork}

We recruited ten participants (five men and five women) between eighteen and thirty-five years old, who frequently use mobile social networking applications in situations of mobility, in particular Facebook. We asked them to wear camera glasses and record their mobile phone activities during their daily commuting for a period ranging from a week up to ten days. Two of them used their cars and the rest used public transportation. This provided us with about twenty hours of recordings. After retrieving the apparatus and the recordings, we synchronized the camera glasses recordings with the screen-capture data to produce the kind of split-screen images shown in Figure 3.2 and throughout this chapter. This was our raw material for the analysis. We then scanned it to constitute collections of gaze-switching events. In five instances we showed one set of data to the user to elicit their own interpretation of it. In order to highlight the potential of our method in a limited space, we will limit ourselves to the analysis of car-driving cases and use the data obtained in public transportation as a way to enrich our findings.

Using smartphones on the move involves the moment-by-moment joint management of mobile communication and transportation. By using our empirical data on the occurrence and placement of gaze switches, we can understand some important aspects of the temporal organization of multiple involvements and shed some new light on the analytically elusive concept of multi-activity.

\section{Gaze switches and the temporal organization of multiple involvements}

In the example we will develop here, the smartphone user is a woman who connects to Facebook while driving. In the first set of data, she comes to a red light, stops (Figure 3.4), looks down toward her lap and away from the road, places her smartphone on the steering wheel and launches the connection to Facebook, so as to check her list of recent posts on her wall (Figure 3.5). Such conduct is illegal in France, but this is not the point here. What will interest us is rather the way she practically manages a dual orientation toward driving and using the Facebook application.

A red light is a constitutive feature of traffic management that is deceptively simple and familiar. It acts as an instruction for drivers to stop for a time that may be roughly anticipated by drivers with enough experience. It does not act by itself. Like the speed bump studied by Science and Technology Studies (Latour 1999), the red light is the surface of emergence of a large and heterogeneous network, an assemblage of people and artifacts "delegating" their agency to the traffic light while remaining at a distance. It is this networked infrastructure that 


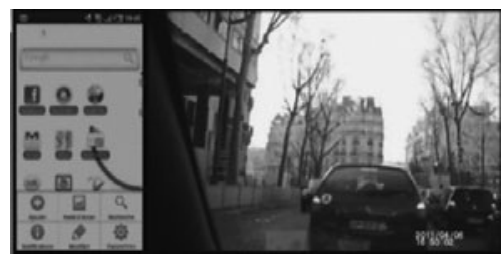

Figure 3.4 Arriving at a red light behind the stopping traffic (@ Christian Licoppe and Julien Figeac).

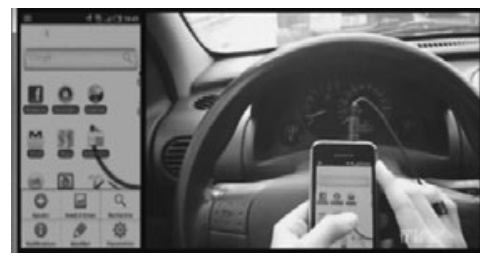

Figure 3.5 Taking such an occurrence as an opportunity to gaze down, put the phone on the steering wheel and launch the Facebook application (@) Christian Licoppe and Julien Figeac).

is "agentive" in the way a red light recognizably and forcefully instructs us to stop. As a visual and semiotic cue which "blackboxes" the networked infrastructure to which it is co-extensive, the red light projects a predictable pause for the surrounding traffic and therefore offers a relevant transition point in the driving activity. It affords a recognizable and convenient slot for doing something else such as picking up one's smartphone.

Such an artifact-mediated temporal patterning of periods of activity and inactivity is not restricted to driving. It is, for example, very common with mobile devices to intervene on the interface, and get a "circular progress bar icon" (Figure 3.6), indicating to the user that the system is doing something and that

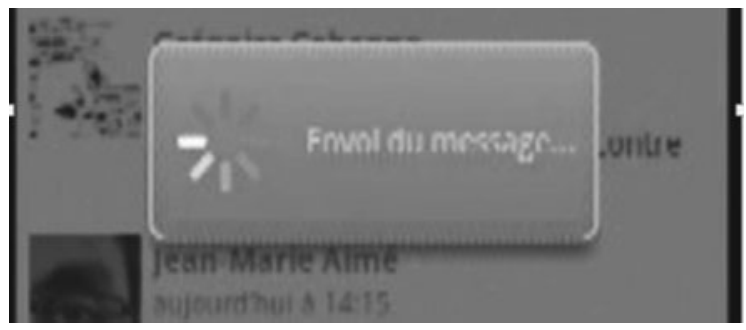

Figure 3.6 A typical visual display for the circular progress bar (@ Christian Licoppe and Julien Figeac). 
until it is done, it is not responsive to the user's actions. The progress bar does not "instruct" the mobile phone user to stop as the red light does because it is grounded in another assemblage of human and material resources. However, like the red light, it projects a pause in the smartphone activity. It works as a "prospective indexical" (Goodwin 1996), signaling that some expectable delay is to be experienced before the expected action is accomplished and the interface becomes actionable again. It somehow "points" toward the future moment in time in which this might happen.

For instance, a few minutes after the first traffic light, our mobile Facebook user drives to another red light at which she looks down at her smartphone again. That she has left it on the steering wheel all along displays her orientation toward multi-activity: It is a way to construct an environment particularly conducive to this, making it easy to gaze swiftly at the mobile terminal and away while driving. At this new red light, she composes and sends a "happy birthday" message to a Facebook friend. When she sends it, she gets the "progress circular bar" icon (Figure 3.7).

Her right hand moves toward the right to engage a gear (Figure 3.8). She takes that smartphone-related pause as an opportunity to engage a gear, an action the preparatory character of which shows evidence of her reorientation toward the traffic about to resume. What is significant here is the way expectable pauses in the smartphone activity are also treated as slots to redirect attention toward driving. Then she looks ahead to the road (Figure 3.9) and sees that the light is still red. This shows that her engaging the gear was not related to any traffic event but indeed to the pause projected in the mobile communication activity.

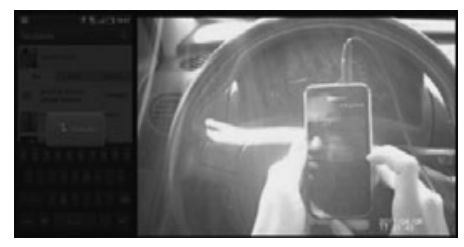

Figure 3.7 Sending a message and getting the circular progress bar (@ Christian Licoppe and Julien Figeac).

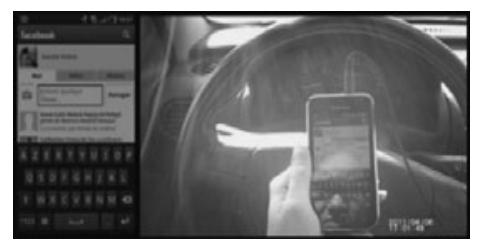

Figure 3.8 The right hand goes to the right to engage a gear (@ Christian Licoppe and Julien Figeac). 


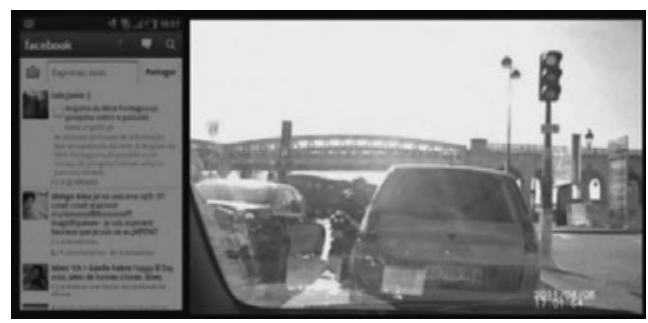

Figure 3.9 The gaze moves up to look at the road and "discover" that the light is still red (@ Christian Licoppe and Julien Figeac).

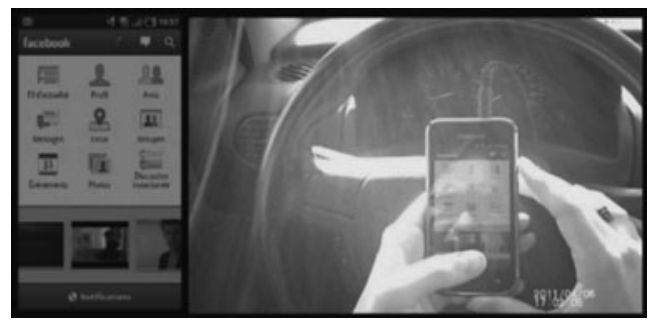

Figure 3.10 She immediately gazes down at the smartphone (@) Christian Licoppe and Julien Figeac).

Characteristically, finding the traffic still at the stop is taken as an opportunity to gaze down at the smartphone again (Figure 3.10), rather than continue on monitoring the road.

Such a pattern of systematic gaze switching at projected pauses in one stream of activity was a recurrent pattern throughout our corpus. When our smartphone users were in the subway, gaze switches away from the mobile phone usually occurred around the moments at which the train was entering into the station or leaving it, whether they were using their smartphones or not. Such a placement probably displays an orientation toward monitoring the progress of the train and the conduct of other passengers coming and going. When they were using their smartphones as well, whenever the circular progress bar appeared, they treated the projected pause as an occasion to gaze away from the phone and to the train environment, whether near a station or not, even if nothing was happening in the subway car at the time.

These observations show that the way we recognize unfolding activities as occasioning and projecting moments of activity or inactivity is a key resource in the management of joint activities. More generally it provides us with an original way to empirically grasp what the experience of multi-activity is about. We can thus pin down an elusive concept which is usually understood either in its 
commonsensical definition of doing two things at the same time (which at a finegrained level is almost never the case) or as the limiting case of such an extreme fragmentation of tasks that switches between activities are so frequent as to become indistinguishable (Kenyon 2010). With our data, we can say that an orientation toward multi-activity becomes manifest when projectable pauses (and possibly other events) in a given stream of activity are systematically oriented to as opportunities to gaze away toward another domain of the environment, relevant to another activity. In that sense, mobile Facebooking and driving, or even mobile Facebooking and taking the subway, are experienced as multi-activity in our corpus. Conversely, being absorbed or engrossed in an activity can be glossed as a tendency to ignore projected pauses in the ongoing activity and remain focused upon it. This tendency may be relevant for situational reasons, as in the example in which mobile Facebooking on a couch becomes engrossing.

Let us note finally that the orientation toward multi-activity in that sense is usually displayed in the organization of the body and the environment. Leaving the connected smartphone on the wheel when driving, or on one's lap when riding the subway, are ways to create an embodied spatial-material arrangement particularly conducive to swift and frequent gaze switches between the smartphone and the mobility-relevant visual domain.

\section{Temporal mismatches in multiple streams of activity}

An orientation toward multi-activity (in our case, mobile communication and transportation) makes the normal "messiness" of the urban environment a constraint and a resource. Urban environments have been described as "messy" to account for the way they are crisscrossed with heterogeneous socio-technical infrastructures and occupied by urban denizens who have been thrown together there and must behave accountably with respect to one another (Bell and Dourish 2007). Such messiness involves bringing heterogeneity and contingent eventfulness to the everyday urban experience. One consequence of this is that for a user engaged in different activity systems, there is no reason for a relevant transition point in one given activity to occur at the exact moment another stream of activity demands action. Different activities will generally project different temporal expectations and mismatched sequential opportunities. We have seen an example of such routine temporal mismatches in the previous section. The driver at the red light who takes advantage of the pause in mobile screen activity after sending a message (Figure 3.8) finds the traffic light still red when she looks up (Figure 3.9) so she looks down at her mobile phone again (Figure 3.10). Being competent at jointly managing the demands of mobile communication and mobility (and more generally any kind of multi-activity) shows one's capacity to handle and minimize the potential consequences of temporal mismatches, so as to perform all relevant activities reasonably well with respect to the demands of the situation.

Such phenomena occur at a micro level in the details of the way the ongoing situation unfolds. They often elicit responsive conduct, which is not reflexive 
and thought about. This is the reason why video recordings of naturally occurring situations such as the ones we are providing here are required to provide better understanding based on observation. For instance, let us return to our mobile Facebook user who had just come to a traffic light and used this as an opportunity to launch Facebook on her smartphone (Figure 3.5). When the traffic resumes at the green light, her gaze can be seen to remain on her smartphone for a lapse of time and not immediately raised toward the street ahead, even though cars visible in the camera glasses (and even more so in her peripheral vision) have started to move (Figures 3.11 and 3.12). That her response is noticeably "delayed" is not just the analyst's opinion since independent evidence of this can be found in the data itself: A driver in the next lane takes advantage of the gap in front of a car created by her delayed response in order to change lanes and take her "slot" (Figure 3.13)

The materialization of such a delay shows how using a smartphone while driving may have consequences on the driving performance. Can we go one step further and provide a more precise interpretation of the reasons for such a delay? This is where the screen-capture data comes in useful. At the moment the light turned green, the driver was in the midst of a scan of her list of Facebook posts: She was scrolling down with her finger, from the most recent (at the top) to older

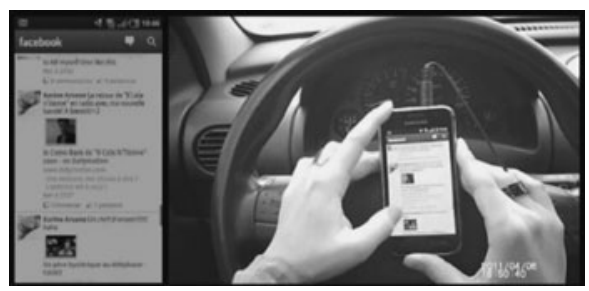

Figure 3.II The driver is scanning down her list of Facebook posts. The sudden motion of surrounding cars is detectable in data through the side window though not visible in the picture (@) Christian Licoppe and Julien Figeac).

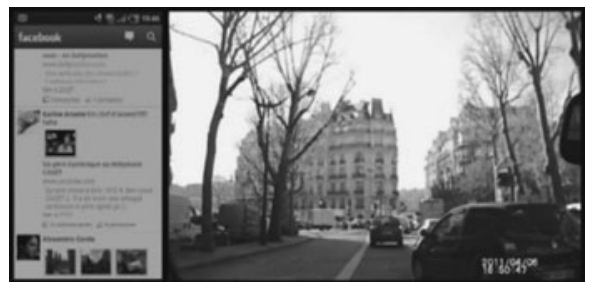

Figure 3.12 She eventually looks up, and a large opening is now visible before her car, materializing the delayed character of her response (@ Christian Licoppe and Julien Figeac). 


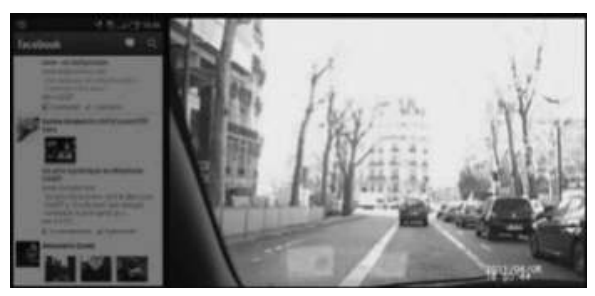

Figure 3.13 The black car on the right "jumps" into the gap, before she eventually starts to move her car forward again (@) Christian Licoppe and Julien Figeac).

ones further down on the touchscreen. It is impossible to say, on the basis of the empirical evidence alone, whether or not she was looking for a specific post (goal-oriented activity) or just waiting for some post on her list to catch her attention (environment-driven activity). Whatever the case, what is significant is that the visual and pragmatic structure of the list does not offer obviously recognizable relevant transition points, the occurrence of which could be taken as an opportunity to gaze away and reorganize one's involvements. One may just scan down on and on until an attention-catching post has been attained, without the list in itself affording any generic and eventful pauses. In the case of a significant event happening outside of the screen, which is at that moment the focus of the visual attention (such as a light turning green), there is a fair chance that the user might remain absorbed in the scanning activity for an extra moment through the sheer inertia of focused attention, even though these "outside" events are demanding some kind of response. Hence the kind of delay we observed when the traffic actually resumed.

Our research methodology not only allows us to observe the way users manage multiple and temporally heterogeneous involvements, but it also provides us here with another grip on the concept of multi-activity. Situations of multi-activity are situations in which users are attuned to the potential sequential implicativeness of events. Yet they are also situations in which temporal mismatches in the occurrence (or projected occurrences) of meaningful events are expected to occur, to be recognizable as such, and to be consequential with respect to the joint accomplishment of the relevant activities. In our example, the delay in the resumption of driving is materialized by the "gap" in the line of traffic in front of the observed driver. It is made consequential by the conduct of the driver on her right who immediately "fills" the space. The expectability of such a sequence is perceptible in the fact it is not noticed or topicalized. It is treated as unremarkable. Even such a routine occurrence provides evidence for the claim that using a smartphone while driving is a form of multi-activity that is potentially consequential in line with social and legal concerns.

We do not claim here that all mismatches and discrepancies in the occurrence of relevance transition points are necessarily detrimental to the accomplishment 
of the relevant activities. We have mentioned above how at a further traffic light the occurrence of a pause in the mobile communication provided the occasion for the same driver to engage a gear before the light turned green, thus displaying her orientation toward multi-activity (i.e., systematically treating pauses in one activity as opportunities to switch one's gaze and/or involvements). The accomplishment of such preparatory action is potentially useful and certainly not disturbing with respect to the impending resumption of traffic. The actual meaning and significance of temporal mismatches in the occurrence of relevant transition points cannot therefore be defined a priori. Such meaning is constructed locally; it is situated and contingent to the details of the unfolding situation. Being a competent user of Facebook on the move relies on being able to manage the consequences of such potential mismatches as smoothly as possible.

\section{Conclusions}

To solve the complex problem of observing the uses of mobile communication on the move, we have developed a portable recording apparatus, based on the combination of camera glasses (worn by the user) and the recording of his or her mobile terminal activity as it appears on the mobile device's screen. We have shown how such a method allowed observation of two kinds of phenomena: (a) gaze switches to and away from the mobile phone (and how they are timed with respect to other perceptible events in the situation); and (b) fine-grained gestures and embodied conduct related to the handling of the mobile terminal and mobile applications in everyday transportation situations.

We have tested this methodology on a sample of mobile users commuting between home and work (by car or public transportation). The observation of gaze switches appears to be a powerful resource in understanding how users try to manage the contingent demands of the various activities they are engaged in. Based on the way users treated projected pauses in the mobile communication or transport activity, we have been able to propose a user-centered definition of what it means for a person to be engaged in a situation of multi-activity: an orientation toward systematically treating projected pauses in one stream of activity as an opportunity to redirect one's gaze and attention toward another stream of activity and vice versa. In that respect, mobile communication in a transport situation is definitely experienced as a situation of multi-activity. As an example, the appearance of the progress bar on screen cues a pause in the smartphone-based activity and is taken as an opportunity to gaze away from the phone toward the mobility-relevant environment.

Events triggering a gaze switch in this way can be described as being sequentially implicative: they are treated as possible slots for reshaping one's attention and involvements in a sequential way. However, their occurrence (or nonoccurrence) is conditioned by what happens in one stream of activity and not necessarily adjusted to the timing and demands of the other relevant activities. Temporal mismatches occur all the time. We have discussed one example in 
depth: a driver scanning down her list of incoming Facebook posts (which did not afford recognizable transition points) recognizably "lagged" when the light turned green. The management of such temporal mismatches is a central feature in the competent handling of multi-activity situations.

The way in which activity environments are navigated projects possible transition-relevant points and therefore plays an important part in the management of multi-activity situations. We may introduce the concept of texture(s) to try and account for the propensity of interfaces to generate or project responseeliciting occurrences in the course of their use. The "pragmatic texture" of an interface describes the way the design of the interface will "afford" interfacemediated events and responses as it is navigated. The "sequential texture" accounts for the propensity of the interface to generate events with sequential implicativeness. An interface can be described as "rugged" (vs. smooth) when it frequently (vs. rarely) generates events of a given type. Textures are relational in the sense that, like affordances, they are as much a feature of the technology design as something relative to a user or a community of users. For instance, in multi-activity situations, users display a heightened awareness of the sequential "ruggedness" of their environments. In these circumstances, they are attentive to the emergence of breaks that appear in smartphone-based activities in order to mobilize them as transition points and mark their involvement in various activity streams. In this case, we argue that the interface can be described as "rugged" because several transition points appear during the use of the device. In conclusion, this chapter reveals the importance of the "ruggedness" of interfaces when used in multi-activity environments. Where safety concerns are critical, it might therefore involve increasing the "ruggedness" of mobile interfaces to augment the frequency of possible transition points and minimize temporal mismatches in the demands of the varied activities the user is engaged in.

Finally, this conclusion runs against more conventional design strategies centered on user-friendliness and the fluidity of the uses as desirable features of mobile media. A more generic approach to multi-activity situations reveals the interest in reconsidering what it means to be "absorbed" by the use of a mobile technology. Recent studies discuss "hyper attention" to account for the ability of users to manage this multi-tasking generated by contemporary media (Hayles 2007). We extend the way in which these studies suggest designing devices for this new economy of attention (Rheingold 2012; Gordon and Bogen 2009) by showing how mobile interfaces supply rhythms that structure the organization of multi-activity and attentional processes engaged in these situations of multitasking. We recommend taking a pragmatic view of the texture of interfaces to rethink their usability and their fluidity from constraints of multi-tasking situations: Users need to gaze away from the smartphone screen to manage other social activities, such as involvement in daily commuting. This phenomenon can be studied by using video recordings because they allow us to observe in depth and analyze pragmatically the management of multiple attention foci and streams of activity as a situated process. Being absorbed or conversely surfing the 
demands of multiple activities appears to be related to the way we recognize or ignore the potential sequential implicativeness of contingent events, act or not upon them, and manage the unremittingly occurring temporal mismatches in the emergence of such events in varied streams of activity. Showing how we do that in practice is a first step toward understanding how we may be held accountable. It is equally a step toward identifying diverse collective orientations toward the management of multiple activities, i.e., recognizing local and emergent cultures of availability and multi-activity.

\section{Notes}

1 See: www.youtube.com/watch?v=Z8GmsKbB9h8.

2 See for instance, "Restaurants' message: no cell phones," New York Times, May 5, 1999; "No rest in peace from mobiles: one fifth of funerals interrupted by mobile phones," Daily Mail, May 15, 2013.

\section{References}

Bell, G. and P. Dourish (2007) Yesterday's tomorrows: Notes on ubiquitous computing's dominant vision, Personal and Ubiquitous Computing, 11(2): 133-143.

Brown, B. and E. Laurier (2012) The normal natural troubles of driving with GPS, in: Proc. CHI 2012, New York, NY: ACM Press, pp. 1621-1630.

Crary, J. (1999) Suspensions of Perception: Attention, Spectacle, and Modern Culture, Cambridge, MA: MIT Press.

Drewes, H., A. De Luca, and A. Schmidt (2007) Eye-gaze interaction for mobile phones, in Proc. Mobility 2007, New York, NY: ACM Press, pp. 364-371.

Esbjornsson, M., O. Juhlin, and A. Weilenmann (2007) Drivers using mobile phones in traffic: An ethnographic study of interactional adaptation, International Journal of Human-Computer Interaction, 22(1/2): 37-58.

Figeac, J. (2012) The appropriation of mobile TV through television preferences and communications networks, in C. Martin and T. Von Pape (eds.), Images in Mobile Communication: New Content New Uses, New Perspectives? Wiesbaden: Springer, pp. 121-141.

Goodwin, C. (1996) Transparent vision, in E. Ochs, E. A. Schegloff, and S. A. Thompson (eds.), Interaction and Grammar, Cambridge: Cambridge University Press, pp. 370-404.

Gordon, E. and D. Bogen (2009) Designing choreographies for the "new economy of attention," Digital Humanities Quarterly, 3.2: 25 paras, available at: http://digitalhumanities.org/dhq/vol/3/2/index.html.

Haddington, P. and Rauniomaa, M. (2011) Technologies, multitasking and driving: Attending to and preparing for a mobile phone conversation in a car, Нuman Сотmunication Research, 37(2): 223-254.

Hampton, K., O. Livio, and L. Sessions (2010) The social life of wireless urban spaces: Internet use, social networks, and the public realm, Journal of Communication, 60(4): $701-722$.

Hayles, K. (2007) Hyper and deep attention: The generational divide in cognitive modes. Profession, 1: 187-199. 
Kenyon, S. (2010) What do we mean by multitasking? Exploring the need for methodological clarification in time use research, International Journal of Time Use Research, 7(1): 42-60.

Lahlou, S. (2011) How can we capture the subject's perspective? An evidence-based approach for the social scientist, Social Science Information, 50(3/4): 607-655.

Latour, B. (1999) Pandora's Hope. Essays on the Reality of Science Studies, Cambridge, MA: Harvard University Press.

Laurier, E. (2000) Why people say where they are during mobile phone calls, Environment and Planning D: Society and Space, 19(4): 485-504.

Laurier, E., H. Lorimer, B. Brown, O. Jones, O. Juhlin, A. Noble, M. Perry, D. Pica, P. Sormani, I. Strebel, L. Swan, A. Taylor, L. Watts, and A. Weilenmann (2008) Driving and passengering: Notes on the ordinary organization of car travel, Mobilities, 3(1): 3-23.

Mann, S. (2012) Wearable computing, in M. Soegaard and R. F. Dam (eds.), The Encyclopedia of Human-Computer Interaction, Aarhus, Denmark: The Interaction Design Foundation, available at: www.interaction-design.org/encyclopedia/ wearable_computing.html.

Mark, G., U. Christensen, and M. Shafae (2001) A methodology using a microcamera for studying mobile IT usage and person mobility, CHI 2001, Workshop on Mobile Communication: Understanding Users, Adoption and Design, Seattle, WA.

Mondada, L. (2012) Talking and driving. Multiactivity in the car, Semiotica, 191: 223-256.

Morel, J. and C. Licoppe (2011) Studying mobile video telephony, in M. Büscher, J. Urry, and K. Witchger (eds.), Mobile Methods, Abingdon/New York, NY: Routledge, pp. 164-182.

Oh, Y.-S. and K.-P. Lee (2005) The development of user observation method and analysis tool in mobile environment-With emphasis on the development of observation method at user's point of view by wearable micro-camera and analysis tool, available at: http://dpl.kaist.ac.kr/web_wiki/images/1/18/ Ohys2005.pdf.

Omodei, M. M., J. McLennan, and P. Whitford (1998) Improving decision making in complex natural settings: Using a head-mounted video camera to improve performance of competitive orienteers, International Journal of Sport Psychology, 29(2): 115-131.

Pink, S. (2007) Visual Anthropology, London: Sage.

Relieu, M. (2002) The "glasscam" as an observational tool for studying screen-based mobile phone uses and management of parallel activities, International Conference on Conversation Analysis (ICCA-02), Copenhagen, May 17-21.

Relieu, M. (2009) Mobile phone "work:" disengaging and engaging mobile phone activities with concurrent activities, in R. Ling and S. Campbell (eds.), The Reconstruction of Space and Time: Mobile Communication Practices, New Brunswick, NJ: Transaction Publishers, pp. 215-230.

Rheingold, H. (2012) Net Smart: How to Thrive Online, Cambridge, MA: MIT Press.

Roto, V., A. Oulasvirta, T. Haikarainen, J. Kuorelahti, H. Lehmuskallio, and T. Nyyssönen (2004) Examining mobile phone use in the wild with quasi-experimentation, Helsinky Institute for Information Technology (HIIT), Technical Report 1.

Yilmaz, A., O. Javed, and M. Shah (2006) Object tracking: A survey, ACM Computing Surveys, 38(4): 1-45.

Zouinar, M., M. Relieu, P. Salembier, and G. Calvet (2004) Observation and capture of multimodal interaction in mobile situations, in Proc.UbiMob 2004, New York, NY: ACM Press, pp. 5-8. 


\section{Chapter 4}

\section{Rerouting borders \\ Politics of mobility and the \\ Transborder Immigrant Tool}

Fernanda Duarte

Scholarship in mobilities and locative media has discussed how the introduction of Global Positioning and Geographic Information Systems (GPS and GIS) in mobile devices has affected the social construction of spaces, places, and locations (de Souza e Silva 2006; Callon et al. 2004; Gordon and de Souza e Silva 2011; Russell 2001; Sheller and Urry 2006; Silverstone and Sujon 2005). From the standard car-navigation GPS device to location-based social networks such as Foursquare, applications of location-aware technologies based on GPS and GIS allow the tracking and tracing of the movement of individuals and things through space and tags them to geographic coordinates. The creation of these systems suggests that the spatial practice of movement is intertwined by a mesh of digital data and physical space, but also suggests a standard of spatial knowledge; a way to know the world spatially that emerges within the technological protocol of GIS and GPS.

Location-aware technologies are put into practice according to forms of knowledge about physical space that endorse specific practices of movement of people and mapping of territories. The pervasiveness of location-aware technologies has created more subtle ways to identify an individual's current location (i.e., track an individual location through self-reported cell phone applications), monitor traveling routes (i.e., through radio frequency identification [RFID] tags in "smart" passports), and create databases about individuals and places (i.e., tag and label individuals using embodied microchips or through biometrics readings). To some extent, it has become difficult to apprehend how, when, and why the collection of data is taking place.

The functional design and application of location-aware technologies emerge within ideologies of technology control and express political and economic meanings about how locational information and personal privacy are negotiated. For example, "checking in" at locations by using applications such as Foursquare implicates an exchange of user data for social and economic currency; the user contributes to her network by adding new information and in return unlocks features that allow for discounts or deals at that location (Frith 2012). The use of GPS ankle bracelets worn by parolees (Shklovski et al. 2009) and biometrics in border control (US General Accounting Office 2002; Ashbourn 
2011) are also examples of a negotiation of technological control and privacy. For the justice system and law enforcement it is crucial that specific populations are immediately located and identified; the use of tracking devices works to map movement and also to tag labels to groups or individuals. In the law enforcement perspective, a GPS ankle bracelet gives the parolee greater freedom, as she is not incarcerated, and also provides law enforcement precise information about the parolee's location. While for the parolee, even though she is not incarcerated, her movements are continuously tracked and, by wearing a device she cannot remove, she is easily identified by others as a criminal offender. The employment of tracking and labeling features in location-aware technologies demonstrates how the use and effects of the technology are taken differently according to which end of the spectrum of technological agency one occupies. Compared to this last example, issues of locational privacy in Foursquare might seem trivial and inconsequential. But similar inner workings of politics, economics, and social forces operate on both negotiations of privacy and control.

Recently, activists have been appropriating location-based media to call attention exactly to these inner workings, to the ways in which the continuities and discontinuities in the navigation of actual and informational spaces are rendered through power relations. In this chapter I investigate one example of activist practice in relation to state borders to demonstrate that the transit between physical locations and mobile devices is inscribed within an arrangement of substantial infrastructure as well as political and economic mechanisms of control. I present a case study of the Transborder Immigrant Tool, an activist project developed by the Electronic Disturbance Theater group (EDT)/b.a.n.g. lab. EDT is a group of artivists/academics currently at the University of California in San Diego-Ricardo Dominguez, Brett Stalbaum, Amy Sara Carroll, Micha Cardenas, and Elle Mehrmand - engaged in developing theory and practice of electronic civil disobedience. The Transborder Immigrant Tool is a software application designed for GPS-enabled cell phones to assist individuals as they navigate through the desert in the surroundings of the US-Mexico border by pointing out the safest routes and the position of water caches. ${ }^{1}$

This chapter is part of a broader study that takes a comprehensive approach to locative media projects that deal with activist and subversive practices around borders and discusses their political implications in a mobile networked society. The study derives from a mobile approach to borders and the concept of politics of mobility (Cresswell 2010) to address two research questions: (1) What politics of mobility are being exercised by the technological protocols that govern border crossing? (2) How do locative media practices exploit breaches of protocol as forms of political resistance and subversion? I present the Transborder Immigrant Tool as an illustration of how locative media exploits breaches of protocol as differential spatial practices.

In a time in which more strict immigration policies in the United States are being adopted - especially regarding the Latin American population - such a project sheds light on the political and territorial matters that ground the delicate 
discussions about immigration policies and border barriers. The imaginary of mobile phones as tools that transcend borders is intermeshed with the discourses of exclusion around the border. Even though the development of mobile technology has created such an imaginary of transnationality, dissolution of barriers and frontiers, the turmoil caused by the development of the Transborder Immigrant Tool unveils the power relations and forms of control underlying technologies of mobility, communication, and border enforcement.

\section{Politics of routing borders}

Despite the static illusion of representations of borders on maps, they are neither inert, arbitrary, nor naturally given. As defined by some contemporary political geographers (Delaney 2005), borders are liminal spaces of political, cultural, and security significance, defined through geographic constraints, negotiated in relation to historical events and through cultural expression. Delaney states that:

Territoriality, then, is much more than [a] strategy for control of space. It is better understood as implicating and being implicated in ways of thinking, acting, and being in the world - ways of world-making informed by beliefs, desires, and culturally and historically contingent ways of knowing.

(Delaney 2005: 12)

Territories are human social creations. They express how individual humans as embodied beings, through cultures and institutions organize themselves in respect to the social and material world. They are more than cultural artifacts, for they are also political achievements, "constitutive of the social orders whose features they express" (Delaney 2005: 10). Territories and their borders are artificially created and are enabled by political and power relationships that define their formation and maintenance.

The emerging field of mobilities studies (Urry 2000; Sheller and Urry 2006; Cresswell 2006, 2010) introduces an approach that also recognizes that the drawing of borders is tied to various formalizations, laws, and protocols and consists of tensions between mobilities and territorialization. Such mobile approaches to the social and political dynamics that occur in borders comes to reinforce a perspective that places itself against sedentarist theories, which according to Sheller and Urry (2006) have been privileged in social sciences. They say that such a-mobile theories frame human identity and experience as grounded in stable territories, thus normalizing particular contingent arrangements of space as static, fixed, and given. Such sedentarist theories thus neglect the ways in which state practices (including borders) emerge out of various arrays of movements of people, objects, ideas, and information. Even though geographically immutable and officially recognized, state borders are actually woven in practices of dwelling, flows of temporary (im)mobilities constituted through economic, social, and political relations. In this sense, a state border is 
an immobile material structure that engenders and embodies representations of power, as it conveys patterns and protocols of movements across territories.

As the motions of the constitution of borders are defined by relations of power, the motions of people's border crossings are equally shaped by these relations. The permeability of borders change according to many issues such as origin (i.e., the US and Mexico border), motives (i.e., tourists vs. immigrants), social characteristics (i.e., marital and employment status, average income), or what Elliott and Urry (2010) define as "network capital." Based on Bourdieu's analysis of cultural and economic capital as the basis for symbolic power, "network capital" stems from the individuals' capacities to sustain a "life on the move" and to endure practices of mobility, such as access to mobile networks that enable one's movement across places and the capacity of coordination with other individuals. The capacity to join "a field of expanding networks" defines how much symbolic power individuals have in the current context of rapid and digitized mobile systems (i.e., international air travel, real-time money transactions, and just in time delivery). In this context, "network capital" as a measure of the ability to navigate the politics of getting documents to move across countries, to access forms of transportation, to provide financial transactions, etc. relates directly to issues of immigration.

The political forces at stake in the constitution of practices of mobility is discussed by Cresswell (2010) as a "fragile" entanglement of physical movement, representations (meanings) of movement, and the experienced practices of moving. All these dimensions together - the corporeal, the discursive, and the performative - constitute a framework for a politics of mobility that allows one to look at different arrays of mobility practices as interrelated scales of power relations in a social situation or as historically situated constellations of mobility throughout time. Technological protocols are also rendered by a politics of mobility and are also modes of exercise of power. As argued by Galloway (2004), protocols are technical procedures as much as norms that dictate behaviors; they are organized ways to exercise power in networks. In the case of the Transborder Immigrant Tool, this relationship is brought to the forefront, as the broad distribution and availability of GPS technology demonstrates how spatial practices are inscribed in informatics. It materializes the continuities and disruptions of what seems to be a global system. As we are reminded by Vukov and Sheller (2013), the production of these practices of im(mobility) and the effects of bordering - as representations of threats and security-require the labor of software developers, infrastructure builders, and guards. The creation of a "smart border" is dependent upon this infrastructure as much as it is dependent upon the data produced by legal travelers and the hidden labor of illegal crossers. The digitization of contemporary bordering practices into surveillance protocols create a "surveillant assemblage" that "operates by abstracting human bodies from their territorial settings and separating them into a series of discrete flows" (Haggerty and Ericson 2000: 606). The creation of "data doubles" of individuals and check points that feed databases (border control or any type of governmental system 
that accounts for location of individuals) promotes the extension and intensification of surveillance to constant monitoring.

As territories are socially constituted based on premises of managing security and risk, borders are cultural, symbolic, and technological performances: "Borders control efforts and are not only actions (a means to a stated instrumental end) but also gestures that communicate meaning" (Raley 2009: 34). The Transborder Immigrant Tool emerges in and through this technological core of border governance. It is the latest project from the Electronic Disturbance Theater (EDT), whose work has continually sought to practice "electronic civil disobedience." One potentially productive route to better understand this project is to look at it as an embodiment of a politics of mobility (Cresswell 2010), as an attempt of subversion of a technological protocol (Galloway 2004) as it approaches the US-Mexico border as a spatial practice (Lefebvre 1992) or a complex social construction (based on values, and the social production of meanings).

\section{The Transborder Immigrant Tool}

The Transborder Immigrant Tool was developed by the Electronic Disturbance Theater in 2007 and it was nurtured by EDT's theorization and practices in electronic civil disobedience since the 1980s. According to Ricardo Dominguez, ${ }^{2}$ the Tool emerged from an ongoing research at CALIT2 that focuses on three main points: (1) theory and practice of electronic civil disobedience and hacktivism, (2) institutional critique and border disturbance art/technology, and (3) nanopoetics and nanotoxicology. The group was particularly interested in using electronic civil disobedience as a research base to expand the earlier traditions of institutional critique and in the training of researchers in art practice and history of activism to imagine the future of these practices. The questions that animated the creation of the project included: What were the implications for an autonomous media practice once it is interpolated into a university system? Did it nurture a practice or did it amplify it in some way? What does it mean to use the practices against the institution using their own systems as primary research? What would happen if you used electronic civil disobedience and hacktivism against your own institution?

The Transborder Immigrant Tool is described as a Mexico/US border disturbance art project that instigates discussions of the physical and virtual limits of this frontier, but also of frontiers of institutional assessment, media commentary, and governmental regulation. In the project's website, ${ }^{3}$ the artivists define this initiative as one that responds to the dangers of border crossing toward the north due to the vertiginous geography of the desert.

The tool consists of an inexpensive cell phone, with a global positioning satellite (GPS) chip, and a custom piece of software. The software was adapted from the psychogeography algorithm "Virtual Hiker" developed by Brett Stalbaum and is available for download at the project's website. ${ }^{4}$ It was designed to 

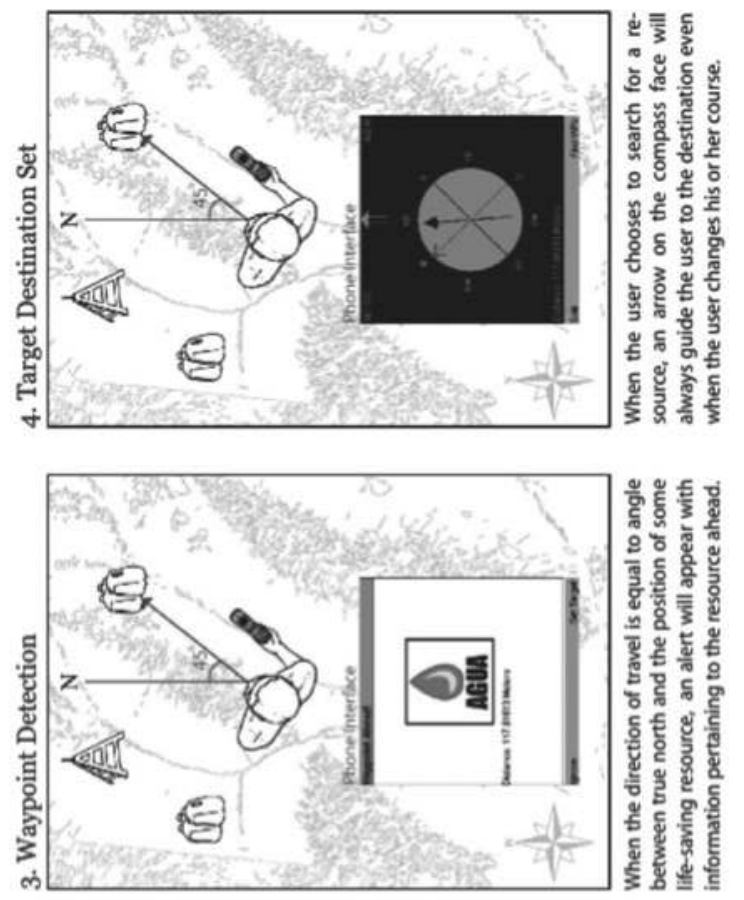

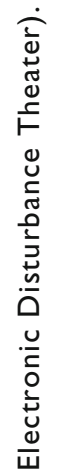
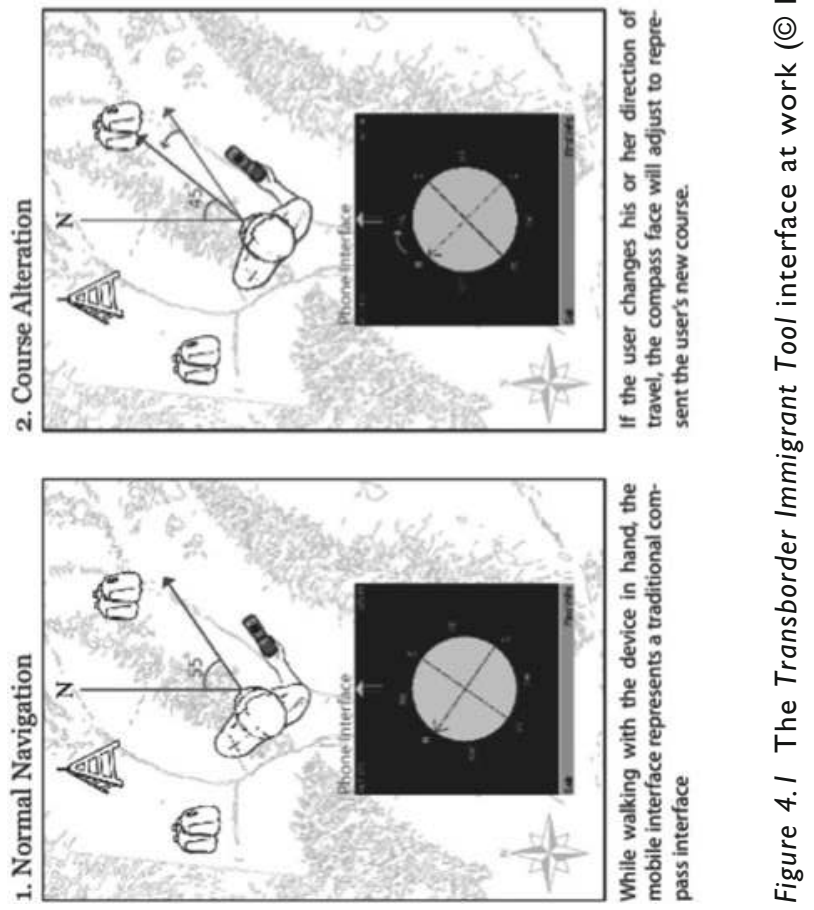
work in inexpensive cell phones and it is a Java-based application that uses the phone's GPS features without needing to send out data through the telephony service operator, which could allow the user to be located. It was adapted to assist border crossers in the Tijuana/San Diego border area as it directs the user of the phone toward the nearest aid site, be that water, first aid, or law enforcement, along with other contextual navigational information. To find water the user can access the visual interface that emulates a simple compass, or say "agua" (Spanish for water) and the phone vibrates more intensively as the user moves closer to the water cache. ${ }^{5}$

According to Ricardo Dominguez, ${ }^{6}$ the Transborder Immigrant Tool was created with the aim of reappropriating widely available technology to be used as a form of humanitarian aid. In 2012, 477 deaths were registered in border crossing attempts from Mexico to the United States, an increase of 27 percent compared to the previous year. Since 1998, more than 5,500 immigrants have died due to loss of orientation in the desert and dehydration. ${ }^{7}$ The design of a haptic interface - with visual, audio and tactile responses - sought to protect the user from the hostile environment and evinces the artivists' concern with the safety of border crossers. The Transborder Immigrant Tool

would not only offer access to this emerging total map economy-but, would add an intelligent agent algorithm that would parse out the best routes and trails on that day and hour for immigrants to cross this vertiginous landscape as safely as possible. ${ }^{8}$

The project was funded by an Arts and Humanities grant from the University of California at San Diego in 2007-2008 and its implementation consisted of five steps:

1 GPS mapping of the Mexican and US sides of the border in order to define the map triangulation coordinates that would guide the development of the software tool.

2 Registration and annotation of occurrences of infrastructure that might interfere against or in favor of the border crossing, for example Homeland Security activities, border patrol, and availability of water and food.

3 Programming of the actual software application.

4 Testing of the Transborder Immigrant Tool in loco.

5 Sharing and distribution of the tool with immigrant communities on both sides of the border.

The project, according to its designers, has clear aesthetic, political, and artistic aims. It aims to serve as a tactical intervention of distraction and disturbance in the supposed order of transnational corridors. The algorithm does not simply carve out safe routes "in terms of a map or a politics" but seeks out what Dominguez calls "the most aesthetic crossing." In addition to the navigational 
capabilities of the Tool, and the humanitarian aid to prevent deaths in the crossing of the desert, the artivists have also added another module to the software in order to promote the dialogue further toward one of hospitality. As the user walks with the tool, after a given temporal interval, a few lines of poetry begin to play. With this gesture, they seek to provide poetic sustenance, to enact a space of hospitality, and to welcome the traveler into a new space. With these temporally triggered poetic interventions, they attempt to reflect on the time of crossing and also to alleviate some of this difficulty. Also, the impulse of radical poetry to the device reinforces the imagery of the tool beyond its geolocation functionality and recharges the immigrants to perceive themselves not as empty shells for labor but also as subjects sensitive to the aesthetic.

Duration is a key concept of performance art and with the shifting roles of performer and audience in contemporary media art works, one can see the trip across the border as a durational walking art piece. De Certeau (1984) discusses how the action of walking manipulates spatial practices as the subject's movement is simultaneously embedded in and appropriating of narratives of the social fabric. The action of walking in this case is also layered with inscribed digital information in physical space as the navigation is mediated by the GPS algorithm. The border crosser produces a sense of place-making that is woven into the physical constraints of the geolocation, shaped by the power relations that position moorings and dwellings of mobility but also emerged within practices of "mobile mediality" (Sheller 2013), the arrangements in which mobile media and locational data are appropriated and contribute to the social construction of public spaces and new infrastructures of mobility.

As an intervention in geographical spaces, the Transborder Immigrant Tool is bound to the physical location of the border at the same time that it introduces desert crossers to the alternative spatiality of the Virtual Hiker algorithm. The production of this alternative spatiality is supported by a notion of hybrid space (de Souza e Silva 2006), which defines that place-making is built upon trajectories generated by the participant. Hybrid spaces emerge out of the relationship between physical and digital spaces, involve mobility and communication and are produced through social and cultural actions. The notion of hybrid space proposed by de Souza e Silva (2006) also requires that (geo)location be recontextualized in ways that invite us to reflect upon the contingent relationship between technological developments, networked social practices, and our own desire to locate and be located. Gordon and de Souza e Silva (2011) define net localities as more than additional layers of information on physical space. They are hybrid localities in transit, not given a priori, but built upon the trajectories users trace as they build connections between each other and physical locations they interact with, let it be physically or remotely. The place-making practice introduced by the Transborder Immigrant Tool corroborates the theoretical framework of hybrid spaces and net localities. As the border crosser's body is doubled with its data correspondent, her identity is constituted through a more distributed agency that is affected by remote iteration and is not limited to a specific location. 
Place-making, in this context, is not tied to the construction of the physicalgeographical space of the nation state territories, but folds into the construction of the double data body of the crosser, as she generates data about her mobility practices. Net localities and hybrid spaces are not bound to the geographical space it refers to, or even within the boundaries of the body's skin, but through distributed agency remotely accessed and prompted from multiple locations.

For Micha Cardenas (Dominguez et al. 2009), the project is concerned with "taking technology and repurposing it ... putting it in a different context and using it in a different way ... taking that GPS technology and making it available to people that really, really need it." In effect, the Transborder Immigrant Tool half conceals the act of assisting migrant crossings inside an art project, while simultaneously making art from the aesthetics of desert crossings. It is this profound uncertainty as to the effects of action that seems to be important in interrupting the rituals of contemporary security. It is not the case that art needs to be close to the observable and situated action easily identifiable as "political" in order to be effective. Indeed, one might say that handing over security technologies to the alien immigrant does little to unsettle the sense of certainty about the border and its technologies. Also that it (merely) points to a particular resolution where more people have access to geolocational technology and are hypothetically able to cross the border. Moreover, the Tool calls attention to the protocols of the securitization of borders, and how they are created to provide "safer" journeys to certain categories of travelers (i.e., jet setters) while forging other categories (i.e., immigrants traveling north) as suspicious and "risky."

\section{Globalization or borderization?}

A discussion about the ways in which the politics of mobility are put in operation in the case of the Transborder Immigrant Tool encompasses practices of movement at different scales and across different dimensions. Border crossing, as a practice of mobility, entails the corporeal experience of movement, as a raw sensorial experience, as much as the social embodied experience. The politics of mobility at work are informed by immigration politics and also by the ecology of the desert, its spatial and geographical conditions. From the motives that trigger the immigrant to cross the border, to the actual tactile experience of the inhospitable desert, to the creation of a hybrid perceptive space (de Souza e Silva 2006), the tool mediates between the physical space of the desert, the GPS coordination of locations, and the immigration policies the tool challenges. More than the phenomenological dimension of the border crossing, I discuss how the articulation of the Transborder Immigrant Tool establishes with ideologies of spatial knowledge, action, and ultimately power when it becomes available and put in use.

In response to my first research question (What politics of mobility are being exercised by the technological protocols that govern border crossing?), I acknowledge that the politics of mobility (Cresswell 2010) that are representative of the conditions of the border crossing in Tijuana/San Diego are 
representative of power struggles that inform regulations on immigration, world politics, communication infrastructure, and protocols for governance of mobility of people. The politics of mobility that engender the Transborder Immigrant Tool unravel and are exposed in media discourses, institutional actions, and governmental regulations.

Cresswell (2010) argues that the practical dimension of mobility is an eternal negotiation between the sensorium capacity of the biological body and the social construct in which this body was conformed and habitualized (in Bourdieu's understanding). The crossing of the border - whether through legal procedures, getting in line in official checkpoints, or illegally facing the aridity of the desert - in each case mobilizes the body to respond to and engage in the tactile and social environment. Moving across borders is an act that implicates not only physical dislocation but also a political ecology built upon the purpose and agency of movement (why does it move? does it move according to its own will?); how movement occurs (speed, frequency, and rhythm); which discourses encourage or censor movement; and how does it feel? (what is the embodied experience of movement?). To look at mobility by narrowing it to physical movement might be enough to measure the amount of movement that occurred in terms of length, frequency, and speed, but it might not reveal how or why it occurred. Looking solely at people's movement across borders could inform us about immigration rates, legal and illegal crossings, but it would not suffice to depict the complexity of motives that animate immigration. The vectors of the politics of mobility (Creswell 2010) in borders are reproductions of historical divides translated in the governance of the border space. Uneven national power relations over capital, race, and other forms of dominance are critical in the establishment of differential (im)mobilities.

In the case of migration from Mexico to the US, it is usually depicted as voluntary, motivated by the possibility of having a better quality of life, and as an escape from an environment of violence, drug trafficking, and economic trouble. In fact, in 2009, a report released by the Pew Research Center demonstrated that most Mexicans (57 percent) believed that life is better in the US and given the chance, one out of three would choose to migrate. In the update of the survey done in 2011, the coefficient fell to 44 percent (Pew Research Center 2011) and the immigration rates fell from more than one million in 2006 to 404,000 - a 60 percent reduction. The report points out that reasons for the decline include increased border enforcement, decline of available jobs in the US, and recent economic growth in Mexico. Even though the migration flow from Mexico to the US is still significantly higher than the opposite, the decrease in flow demonstrates that the ideal of the US American territory as a "land of opportunities" still resonates but no longer reigns unquestionable. It now lies side by side with the recognition of US industry's dependency on foreign cheap labor and a rising Mexican pride regarding cultural roots and national identity.

The Transborder Immigrant Tool exposes the occluded conditions of mobility across the border and questions the differential rights to mobility and uneven 
agency regarding voluntary movement, when locational information belongs not only to law enforcement but also to immigrants. By providing access to an information system, the Transborder Immigrant Tool aims at disturbing the hegemonic power dynamics that populate the "northern cone's imaginary about who has priority and control of who can become a cyborg or "trans' human."10 While north-to-south movement operates more freely in regard to mobility of people, goods, and even waste, as the Transborder Immigrant Tool reappropriates a "global positioning system" and transforms it into a "global poetic system," it empowers Mexican immigrants with the agency of inventive action in space. By developing a GPS-based algorithm, EDT was able to develop a tool that parses out the difficult geography of the physical landscape of the desert and of the political terrain in which immigration is rooted.

The Tool was made available for download from the internet in 2008 and it soon got the attention of media art festivals and Hispanic culture organizations. When the story that was featured on the underground New York City Brooklynbased Vice Magazine went viral, the Transborder Immigrant Tool also got the attention of mainstream media, the California State Education system, and the Federal Government.

It might be because territory in a modern system of thought is tightly bound to the notion of "sovereignty" as a particular form of power and authority tied to nations (Sassen 2008), that this project was labeled by right-wing media as a result of possible misuse of public funding and even as an alarming threat to the United States National Security.

In Glenn Beck's television show at Fox News, ${ }^{11}$ the headline of the story on the Transborder Immigrant Tool read: "UCSD professors: Dissolve U.S.-Give GPS phones with explicit poetry to illegals for border crossing." Beck's monologue starts by entitling his view as the undebatable truth and asks for divine providence to convince the audience that UCSD employees, with the audience's "hard earned dollars," are involved in supporting illegal immigration. He claims that the funding of the project — and others like it — must be interrupted and that the professors involved in the development of the Tool should be fired. He then addresses the fact that their tenure track stops them from being terminated and remembers the days in which

good old America walked you through walls of fire to make sure we weren't funding Hamas or Hezbollah.... There are a lot of universities that are as dangerous with the indoctrination of the children as terrorists are in Iran or North Korea.

\section{Dominguez's declaration that}

in terms of immigrant rights ... the question is to move the kind of anchor of civil disobedience within the kind of over-fetishized notion of a single sovereignty of a nation but to look at a larger trans-track a larger transsociety that is emerging that also should have global rights ${ }^{12}$ 
was radically interpreted by Beck as saying that the artists wanted to "dissolve the United States."

Because of Glenn Beck's speech against the Transborder Immigrant Tool, the Electronic Disturbance Theater group got considerable media attention and often the questions regarding the use of the Tool were not related to the humanitarian aid on which EDT was focused, nor even on Mexican immigration. The most recurrent topic was terrorism. When asked about the consequences of the Tool falling into the hands of Al-Qaeda, Dominguez replied:

Well, if Al-Qaeda really wants to cross the U.S.-Mexico border, they can go to Best Buy in Tijuana and pay \$59 for a GPS that will give you very clear, very precise locations of wherever you want to go. [TBT] is not going to help you cross into Phoenix or any place. What happens is that, if you're dying, you turn it on. About four to five hours are on this phone. And basically, it will try to lead you directly to the closest water cache last known within the last four days left by Water Station Inc. or Border Angels. ${ }^{13}$

Even though the Transborder Immigrant Tool was the winner of the Transnational Communities Award, Beck's editorial echoed in the Congress and Republican members lobbied for an investigation regarding the legality of the actions carried out by the UCSD faculty. The FBI Cyberdivision, after looking at the documents and archives of the Transborder Immigrant Tool, reached the conclusion that the development of the project was part of Dominguez's Research Plan of Work, which had been previously evaluated and authorized by the university at the moment of his hire and again when he was granted tenure. For these factors, among others, the Congressional investigation concluded that there was no need to pursue the investigation any further. The professors involved in the development of the project were also submitted to an internal investigation at the University of California in San Diego. The investigation aimed to learn whether the use of the university's funding, public grant money, was indeed financing illegal immigration. After six months of investigation, on July 21, 2010, the UC auditors finally reached the conclusion that "neither University funds nor effort were used inappropriately during the development of the TBT or the Project."14 The investigation was finally brought to an end.

In response to my second research question (How do locative media practices exploit breaches of protocol as forms of political resistance and subversion?), I acknowledge that the tool repurposes functional geolocation to provoke norms and protocols. The practices of hacktivism and locative media are performative actions more than constituted objects; fleeting events rather than advocates of permanent change; disturbers of protocols rather than resetters of the protocol. This demonstrates that the goal is not to destroy control but to figure out the breaches and gaps in the current protocols. EDT demonstrates that their artivist practices are as much aesthetic as they are political. The gesture the Tool engenders is an affective gesture of art as much as it is an effective political action of 
code switching; even when it does not reset the protocols it is part of, it still disturbs them.

Nevertheless, the action of figuring out potential exploits and ruptures demands constant engagement, critical operations, and experimentation by whoever decides to test the limits of protocols. The Tool assumes a relational aesthetics as its poetics demand the actants to become more attentive to their action in space and to the protocols that sustain the apparatus in which they are entangled. When pushing protocols to the limit and hypertrophying technology, the actants are incited to assume a critical attitude regarding their role as a political subject woven into different scales of power relations. Dominguez concludes:

The Transborder Immigrant Tool can be seen as part of a larger shift from Tactical Media to Tactical Biopolitics. While media artists of the late nineties and early two thousands were often concerned with the political potentials opened up by cheaper access to media technologies, we are interested in the political potential opened up by technologies which can serve to improve people's lives directly, including medical technologies and safety devices such as GPS tools.

(Dominguez et al. 2009)

By reappropriating GPS into a global poetic system, the Transborder Immigrant Tool falls into multiple dynamics involving biopolitics: of the coyote operations in Mexico that charge to guide the immigrants during the border crossing, of narcotrafficking that counts on the financial necessity of these people to entice them into criminal practices, and also of the availability of cheap labor in the US.

Through the technical capabilities it appropriates, it is also tied to the power relations that enable technological infrastructure. The fact that the telecommunication infrastructure is more robustly installed in the northern hemisphere ensures a greater bandwidth capacity, and therefore a more stable grid to this part of the globe, yet causes international information flow to be inevitably routed through the northern internet network. This geographical concentration of the technological mediation of most online data exchange should be seen not as an independent matter of technical affordances, but as a continuum of power relations in the world's political economic scenario.

An interpretation of networks as fluid mobilities, or in the other extreme, as absolute antinomies such as global and local, or even (reducing the agency of cultural and technological manifestations) as mechanically programmed reactions, inhibits a clearer understanding of the complexity of a global network. The concentration of technological infrastructure in the northern hemisphere should tell us not only about how this difference impacts on technical capacities to host and engage commercial transactions, but also how it contradicts the morphology of a global, identical, symmetric network. The information flows are not smooth. 
The centrality of infrastructure of data proves that the norms that regulate the actual space of the borders are under the same normative regime that sets boundaries to the transfer of data packages in GPS-enabled cell phones, that regulates mobility of people, and are the same ones that the Transborder Immigrant Tool challenges.

The battle the creators of the Transborder Immigrant Tool fight is material and symbolic, fought on the very "political stage" where power is exercised. According to Ulrich Beck (2002) there is a dialectical relation between the transborder condition of the immigration flux and the unequal experience of being victimized by global issues. Globalization as a homogeneous depiction of the world is a fallacy, since in the peripheral countries, "world risk society appears not as an endogenous process, which can be fought by means of autonomous national decision-making, but rather as an exogenous process that is propelled by decisions made in other countries, especially in the so-called center" (Beck 2002: 42). Even though the solution of extra-national problems requires global cooperation (the issues of scarcity of natural resources, for example), in the distance between the proposition of the solutions and their actualization lies a great potential for conflicts.

In April 2013, the US Senate passed the Border Security, Economic Opportunity and Immigration Modernization $\mathrm{Act}^{15}$ that calls for a $\$ 46.3$ billion investment in border control. This investment includes maintaining at least 38,405 full-time border patrol agents (twice as much the current operating force), the building of 1,125 kilometers of fencing, and the implementation of surveillance technologies such as ground sensors, portable contraband detectors, and radars. The escalating control of borders, especially the United States and Mexico border, and its performative effect in actually deterring the illegal trespassing demonstrates a will to exercise and feign control over what might not be controllable. The measure of success of the control of the borders is not to be measured by the numbers of illegal immigrants that were caught by the border patrol, but the imaginary number of illegal immigrants that were coerced by the risk discourse and did not even dare to try.

\section{Conclusion}

Locative media, apart from investing people, location, and mobile devices with great (and efficient) connectivity, also problematizes the use, limitations, and boundaries of space beyond the discussion of usability in the field of HumanComputer Interaction. Questions dealing with locative media must go beyond understanding physical reality input as an additional layer to mediate our networked condition, and watch for how the transit between physical locations and mobile devices is inscribed within an arrangement of substantial infrastructure, political, and economic mechanisms of control. The actual space is not only a physically constituted concrete space, but a space with regulations, norms, and rules. 
The stressing of the forces that engender a politics of mobilities evinces aesthetic and political potentials in the use of location-based media. Projects such as the Transborder Immigrant Tool work as a relational apparatus that articulates machinic arrangements and aesthetic, social, and political encounters. The Tool not only unveils the territorial logic of the border, which is always there, but also the power relations and forms of control underlying technologies of mobility, communication, and border enforcement. It constitutes a model of micropolitics in practice because their subversive and critical poetics invents alternative lines of flight, and proposes temporary and nomadic constructions without making claims for a revolutionary transformation of reality or utopian designs. Locative media thus aim to upset the consolidated perspective of territories and to call attention to the power relations that shape and constitute territories. EDT negotiates its poetics within the aesthetic-political rationale of tactical media (Raley 2009), since they do not aim to develop a transcendental cultural or political project preconceived in a historical evolution.

Locations, spaces, and territories are always regulated by laws, rules, and protocols and consist of tensions between motions of (de-) and (re-)territorialization. The understanding that territories are both social constructs and can be understood as hybrid spaces-built upon the overlapping of information flow and actual geography - allows us to perceive how the protocols that regulate the dynamics of these spaces are constituted. Even though open markets and the constant flow of capital is a premise to the neoliberal capitalism in the era of ultimate globalization, the role of nation states has been exercised not in the direction of freeing the flow of transactions. Instead they have become even more sophisticated "gatekeepers" in the governance of the flows, in the exercise of protocols; especially when it comes to the (im)mobility of people. Further research should focus on how locative media informs theoretical research about the practices of mobility but also informs decision makers, regulating agencies and governmental agents about the politics of mobility at work.

\section{Notes}

1 See: http://post.thing.net/node/1642.

2 Personal communication with Ricardo Dominguez, September 29, 2011.

3 See: http://bang.transreal.org/transborder-immigrant-tool/.

4 The "Virtual Hiker/Walking Tools" algorithms and the Transborder Immigrant tool are open platforms and can be reappropriated to assist border crossings in other locations as well. Download available at: www.walkingtools.net.

5 The group paired with the NGOs Border Angels and Water Station Inc. that provide water caches in the desert. EDT mapped the location of these caches and provided the information with the tool.

6 Personal communication with Ricardo Dominguez on September 29, 2011.

7 Available at: www.nfap.com/pdf/NFAP\%20Policy\%20Brief\%20Moral\%20Case $\% 20$ For\%20a\%20Temporary\%20Worker\%20Program\%20March\%202013.pdf.

8 See: http://bang.transreal.org/transborder-immigrant-tool/.

9 Personal communication with Ricardo Dominguez on September 29, 2011. 
10 Ricardo Dominguez in interview with Leila Nadir. Available at: http:/hyperallergic. com/54678/poetry-immigration-and-the-fbi-the-transborder-immigrant-tool/.

11 The video with Glenn Beck's opinion on The Transborder Immigrant Tool is available at: http://video.foxnews.com/v/3955297/transborder-immigrant-tool/.

12 See: http://web.archive.org/web/20120830062232/http://bang.calit2.net/tag/ transborderimmigrant-tool.

13 See After the storm, September 8, 2010 at: www.sdcitybeat.com/sandiego/printarticle-8159-print.html.

14 See: http://web.archive.org/web/20120627081843/http://bang.calit2.net/xborder/.

15 See: www.immigrationpolicy.org/special-reports/guide-s744-understanding-2013-senateimmigration-bill.

\section{References}

Ashbourn, J. (2011) Guide to Biometrics for Large-Scale Systems Technological, Operational, and User-Related Factors, London: Springer.

Beck, U. (2002) The terrorist threat: World risk society revisited, Theory, Culture \& Society, 19(4): 39-55.

Callon, M., J. Law, and J. Urry (eds.) (2004) Absent presence: Localities, globalities and methods, Special Issue of Environment and Planning D: Society and Space, 22(1): 3-190.

Creswell, T. (2006) On the Move: Mobility in the Modern Western World, New York, NY: Routledge.

Cresswell, T. (2010) Towards a politics of mobility, Environment and Planning D: Society and Space, 28(1): 17-31.

de Certeau, M. (1984) The Practice of Everyday Life, Berkeley, CA: University of California Press.

Delaney, D. (2005) Territory: A Short Introduction, Malden, MA: Blackwell Publishing.

de Souza e Silva, A. (2006) From cyber to hybrid: mobile technologies as interfaces of hybrid spaces, Space and Culture, 9(3): 261-278.

Dominguez, R., M. Cardenas, A. S. Carroll, and B. Stalbaum (2009) The Transborder Immigrant Tool: Violence, solidarity and hope in post-NAFTA circuits of bodies electr(on)/ic, MobileHCI, September 15-18, 2009, Bonn, Germany, available at: www. uni-siegen.de/locatingmedia/workshops/mobilehci/cardenas_the_transborder_immigrant tool.pdf.

Elliott, A. and J. Urry (2010) Mobile Lives, New York, NY/Abingdon: Routledge.

Frith, J. (2012) Constructing location, one check-in at a time: Examining the practices of Foursquare users, doctoral dissertation retrieved from Dissertations and Theses database: www.lib.ncsu.edu/resolver/1840.16/8064.

Galloway, A. (2004) Protocol: How Control Exists After Decentralization, Cambridge, MA: MIT Press.

Gordon, E. and A. de Souza e Silva (2011) Net Locality: Why Location Matters in a Networked World, Boston, MA: Blackwell-Wiley.

Haggerty, K. and R. Ericson (2000) The surveillant assemblage, British Journal of Sociology, 51(4): 605-622.

Lefebvre, H. (1992) The Production of Space, London: Blackwell.

Pew Research Center (2009) Troubled by crime, the economy, drugs and corruption. Most Mexicans see better life in the US - One in three would migrate, Pew Global 
Attitudes Project, research report released September 23, 2009, available at: www.pewglobal.org/files/pdf/266.pdf.

Pew Research Center (2011) The Mexican-American boom: Births overtake immigration, Pew Hispanic Center, research report July 14, 2011, available at: http://pewhispanic. org/files/reports/144.pdf.

Raley, R. (2009) Tactical Media, Minneapolis, MN: University of Minnesota Press.

Russell, B. (2001) The HeadMap Manifesto. Available at: http://technoccult.net/ technoccult-library/headmap/.

Sassen, S. (2008) Territory, Authority, Rights: From Medieval to Global Assemblages, Princeton, NJ: Princeton University Press.

Sheller, M. (2013) Mobile mediality: Locations, dislocations, augmentation, in S. Kesselring, G. Vogl, and S. Witzgall (eds.), New Mobilities Regimes: The Analytical Power of Social Sciences and Arts, Aldershot: Ashgate.

Sheller, M. and J. Urry (2006) Mobile Technologies of the City, New York, NY: Routledge.

Shklovski, I., J. Vertesi, E. Troshynski, and P. Dourish (2009) The commodification of location: Dynamics of power in location-based systems, UbiComp 2009, September 30-October 3, Orlando, Florida.

Silverstone, R. and Z. Sujon (2005) Urban tapestries: Experimental ethnography, technological identities and place, available at: www.lse.ac.uk/media@lse/ research/mediaWorkingPapers/archive/ewpNumber7.aspx.

The Transborder Immigrant Tool, available at: http://bang.transreal.org/transborderimmigrant-tool/.

Urry, J. (2000) Sociology Beyond Societies: Mobilities for the Twenty-First Century, New York, NY: Routledge.

US General Accounting Office (2002) Technology Assessment: Using Biometrics for Border Security, GAO-03-174, November, Washington, DC.

Vukov, T. and M. Sheller (2013) Border work: surveillant assemblages, virtual fences, and tactical counter-media, Social Semiotics, Special Issue: Charting, tracking, and mapping: New technologies, labor, and surveillance, 23(2): 225-241. 
This page intentionally left blank 
Part II

Performing location, place-making, and mobile gaming 
This page intentionally left blank 


\section{Chapter 5}

\section{Online place attachment Exploring technological ties to physical places}

Raz Schwartz

@IAmAru: "I feel like I'm betraying my@Foursquare mayorship by going to the other Starbucks" 1

For Aru, the user behind the Twitter handle@IAmAru, being the Foursquare mayor of a specific Starbucks carries special meanings. Although all other Starbucks have the same décor, same menu, and same background music, he continuously chooses to go to this particular one just to maintain his virtual mayor title. But@IAmAru's choice of words is not accidental. "Betraying” is a strong, emotionally charged word mostly used in relationships between two individuals. Although in this case, it is utilized to portray a special connection between this user and a specific branch of the famous coffee shops chain.

One might say there is nothing exceptional in Aru's statement as we all have special ties to certain physical places. However, as the introduction of new online location-based services like Foursquare set the ground for hybrid, net locality (Gordon and de Souza e Silva 2011) interactions that carry the influences of both the online connection and the offline presence, we must ask ourselves, what are the elements that "online connect" someone to a certain physical place? Why do people, like @IAmAru, feel an intimate attachment to a specific place by using these technologies? And which theoretical frameworks can we apply to the study of this attachment?

These questions are the main subject of inquiry in this chapter, as I examine the tensions and implications of online interactions to local connections between people and places. Although in recent years there is a growing body of literature that examines location-based social networks, these studies mostly focus on studying the affordances of this technology in regard to interactions between different users. The studies that did look at the connections between users and places explored how, for example, location-based social network users assign information to physical places, and "read and write" locations (de Souza e Silva and Frith 2010, 2012; de Souza e Silva 2013) but did not examine the ways these interactions instrument and maintain attachment between people and places. In this chapter I study these activities through the lens of "Place Attachment" (Low and Altman 1992), an interdisciplinary theory used to study people's ties to 
physical places. By analyzing users' interviews, press coverage, and software interface, I set out to examine the role that checking in to places, obtaining mayorships, leaving notes and tips, and labeling public venues has for our connection to the physical places. The main contribution of this work to current literature is therefore with the introduction and application of Place Attachment to the examination of location-based online social interactions.

This chapter begins with a literature overview of mobilities and locationbased technology research along with a description of the Place Attachment theory. This body of work frames my research questions as part of the growing efforts to study social interactions that are mediated by mobile devices and online social networks. Then, I describe the methodology and the results of a series of interviews with twenty-five Foursquare users through examining three categories: place-naming, ownership, and events. I present several examples and discuss how these interactions are framed within this theory. Finally, I argue that these interactions are part of a wider notion of "Online Place Attachment," an online-offline personal connection to physical spaces mediated by locationbased technology.

I focus in this work on activities that take place on Foursquare, one of the most popular location-based social networks. Foursquare was first launched in March of 2009 and offers its users to "check in" to the places they visit and instantly share that information with their friends. The application is designed for use with smartphone devices as the location data is gathered automatically through the device's GPS component. In January 2013 the company reported that the service has over thirty million users worldwide, making it one of the largest location-based services in the world. ${ }^{2}$

Every day, millions of users share their endeavors with their friends and explore new places through Foursquare in their local surroundings as the application is designed to encourage the sharing of local knowledge. For example, when a user launches their application they can choose if they would like to check in or just see their friends' activities and explore nearby places.

When checking in, the application finds the user's exact location and creates a list of nearby venues. The venues list is based entirely on user-generated content, which means that if a place does not appear on the list, the user can independently add it. This venue will later appear when they or any other user will try to check in at that location. When the user checks in they can decide if they would like to share it with their friends on Foursquare, Facebook, or Twitter or just stay "off the grid."

Moreover, the application offers its users several other tools to supplement their activity. Among these are tips, likes, pictures, and lists. In this way, users can share their recommendations and experiences through the tips option that will be displayed to other users who will also check in to that venue. Users can also take pictures and add them to the location they visit so other users can also see them. "Like" is an option that was added only in later versions of the application and allows users to annotate a place by "liking" it. Lists provide users a way 

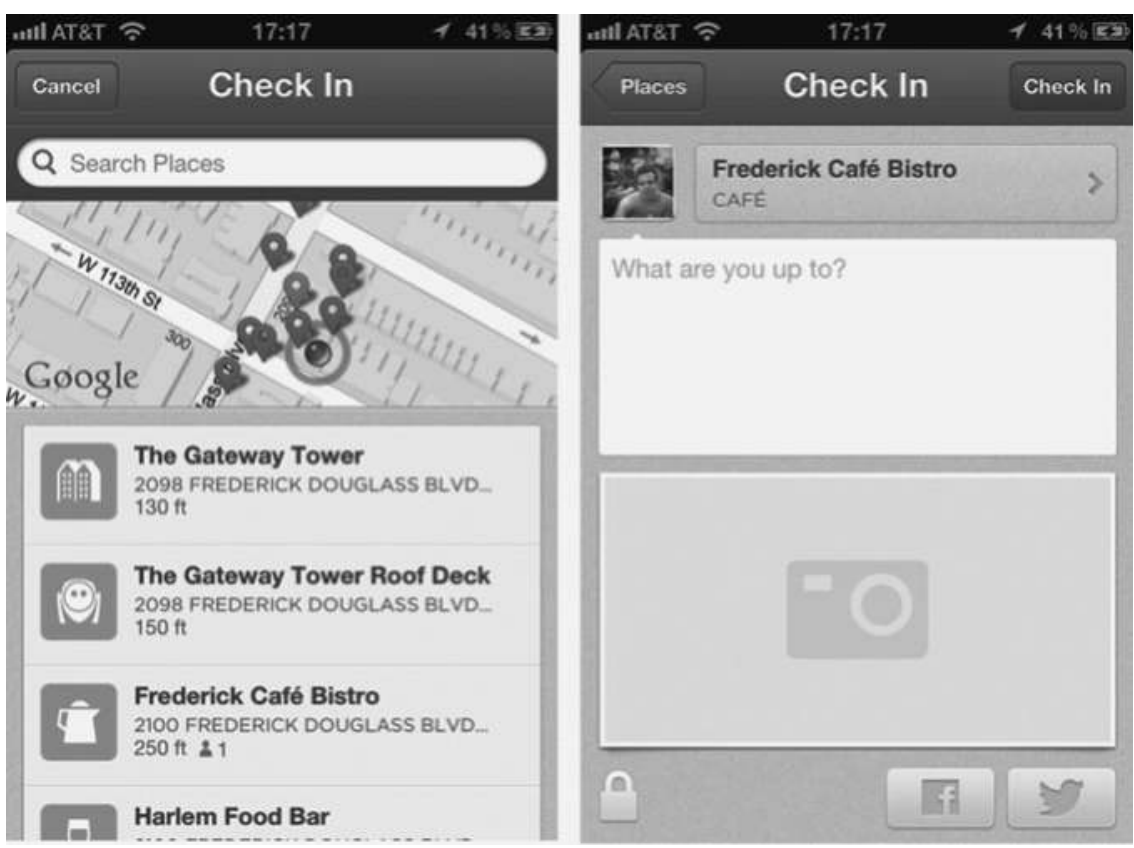

Figure 5.I Check-in screens (@ 20I4, Foursquare Labs, Inc).

to group several different places under some kind of a commonality like "My Favorite Hummus Places in NYC."

In addition, users can also utilize the application to explore places in their locality. By clicking the "Explore" tab on the application, users can get recommendations on places to go based on various categories such as food, coffee, sights, etc. These places are organized by a "curated social graph," a method by which the application can prioritize venues in close proximity and recommends places that match the users' personal preferences based on past activity and friends' check-ins.

In 2012 Foursquare launched a redesigned version of the app that included a new "Friends" tab that operates as a timeline of friends' activity. In this tab users can see these activity updates organized from the most recent backwards. On the timeline, users can interact, "like" other users" check-ins, view their photos, and read the tips and lists they added.

In addition to sharing the location of the user, viewing other users' activities and exploring places nearby, the service also utilizes game mechanics to motivate its users to compete with each other and earn virtual titles and badges. In this way, users can get points, badges, and "mayorships" of specific venues that were visited most frequently in a certain time period.

Foursquare therefore offers its users ways and tools to not only interact with their friends or other Foursquare users on the go but also to document the 
physical places they visit. Since the emergence of this technology, these types of interactions were the subject of inquiry of researchers from various fields as I will describe in the following section.

\section{Mobile technology and physical places}

Mobile technology has changed the ways we think about our relationships between people, actions, and space (Dourish 2006; Ling 2004). We now interact in digital networks rather than the previous door-to-door, home-to-home communications (Wellman 2001), using various technologies like mobile phones and social media networks to manage relationships with individuals in a context that is not set to a specific physical location. We can therefore see a shift from a traditional mass media culture to a new personal communication society (Campbell and Park 2008). This change is also reflected in our physical environment, as people now use mobile communication to appropriate public spaces for personal purposes. As a result, we now have new temporalities and spatialities for public participation (Sheller 2004). Public participation is now enacted both online and offline by demonstrations in physical places such as Zuccotti Park, Tahrir Square or Rothschild Boulevard as well in the Twitter feed with the \#occupy in the US, \#jan25 in Egypt and \#J14 in Israel.

In other words, the introduction of mobile technology fosters various forms of interaction that did not exist before and produce a sense of self for users, which is derived from the intersection of the virtual and physical realms (Farman 2011). And although these options may not increase our spatial awareness and encourage meeting more people (Sutko and de Souza e Silva 2011), this move to networked individualism as a looser, more fragmented set of social networks that provide on-demand support, opens new ways of interaction between people and places (Rainie and Wellman 2012). Places are no longer only appropriated for a specific group.

But despite notions that treat these places as non-places (Augé 1995), namely places that are losing their significance and meaning to us through the development of communication technology, previous works found that using locationbased technology can actually promote users to experience their locality through mobile games (de Souza e Silva and Hjorth 2009), facilitate the creation of third spaces (Humphreys 2008, 2010; Schwartz 2013) as well as better represent the different dynamics of the local social groups that operate in a city (Cranshaw et al. 2012).

These abovementioned works are only a small part of a large corpus of academic efforts that investigate interactions between people and places. For example, Place Attachment is an interdisciplinary theoretical concept that was conceived during the late 1980s and originated from different studies in anthropology, architecture, family and consumer studies, folklore, psychology, sociology, and urban planning. The term represents a symbolic relationship that is created by people who give culturally shared emotional meanings to a particular 
place. This in turn is argued to provide the basis for both the individual and a group understanding of their local surroundings.

Ever since then, there has been extensive research work done in the study of the connection between people and places through this prism. In her work, Low (1992: 166) explains Place Attachment as following "the symbolic relationship formed by people giving culturally shared emotional/affective meanings to a particular space or piece of land that provides the basis for the individual's and group's understanding of and relation to the environment."

Moreover, Low describes a theoretical typology and provides several factors that create place attachment such as narrative linkage, economic linkage, linkage through cultural events, loss of land, and more. In her work, she argues that people's linkage to places can be traced through various factors such as the vehicle of story, place-naming and language, ownership of land, and participation in celebratory events such as parades or festivals; these all form place attachment to the venues we frequent.

Following this typology, various works applied the Place Attachment theory to the study of the critical role of public plazas (Low 2000), the importance of Place Attachment to community participation and planning (Manzo and Perkins 2006; Riger and Lavrakas 1981), and the proposed use of Place Attachment as a tool for neighborhood revitalization (Brown et al. 2003). Milligan (1998), for example, sees Place Attachment as comprised of both past interactions and potential interactions. In this way, the emotional connection that is created between people and places is constructed of both the memories associated with it and future experiences that we might have there.

As Foursquare interactions take place both online and offline, we must utilize the existing research that examined the connection among people and places as well as the ways in which location-based technology promotes local social interactions. Only by integrating these bodies of research can we examine what the elements are that connect someone to a certain physical place. Why do people feel an intimate attachment to a specific place by using these technologies? And how can a theoretical framework like Place Attachment be applied to the study of these interactions?

\section{Methodology}

This chapter is based on the analysis of participant observations, in-depth users' interviews, and media coverage of the location-based social network Foursquare collected through a six-month period from September 2010 to February 2011. I conducted twenty-five in-depth, semi-structured face-to-face interviews with Foursquare users from three cities in the United States (New York City, Pittsburgh, and San Francisco), representing accordingly the East Coast, middle-east USA and the West Coast. The participation requirement for interviewees was to have used Foursquare in a specific area for a period of at least three months and with an ongoing activity of at least three days a 
week. These criteria ensured that the participants had a reasonable chance to interact with friends, explore places, and possibly obtain badges and mayorship titles.

The users were recruited by sending out Twitter messages to my followers asking to retweet the message to their own followers. The message linked to a webpage inviting people to participate in a study that focuses on "how people use Foursquare." These Twitter messages were retweeted by several people to an audience of approximately 2,000 people. I received messages from twentyseven people expressing their willingness to participate, finally resulting in thirteen users that met my participation requirement.

In addition, I used Foursquare's website to identify fervent users by the number of mayorships and titles they held. I contacted these users through their Twitter and Facebook accounts with a request to participate in my study. Out of the twenty people I contacted, twelve agreed to take part and share their experiences.

I conducted my fieldwork in Pittsburgh, New York City, and San Francisco; the demographics of this study consist of twenty-five Foursquare users (thirteen men and twelve women) ranging in age from twenty-four to thirty-five living in Pittsburgh $(n=8)$, San Francisco $(n=7)$ and New York City $(n=10)$. The users I interviewed had a friends network ranging from twelve to 117 friends. All interviewees received $\$ 10$ compensation for their participation.

The interview protocol was structured from open-ended questions investigating the nature of the social interactions people experience when using Foursquare. More specifically, the interviewees were asked about various topics such as their check-in practice (type of place, hours of the day, etc.), motivations, interactions with friends and strangers, place attachment, privacy concerns, and more.

All interviews, which lasted approximately an hour and a half, were conducted and recorded face-to-face and later transcribed. In addition to the interviews, I also documented in detail my use of the application during a one-month period (March 2011), which included both private and group activities.

For this study I conducted issue-focused coding (Weiss 1994) of the interviews based on three of the categories identified by Low (1992: 166): Placenaming, Ownership, and Events. I then did more in-depth thematic coding of the data within those broad categories to identify potential patterns and common themes in discussions about physical venues and social interactions using Foursquare.

\section{Results}

While coding the data according to the three categories, I noticed several unique practices that described the connection between the people and the places they visit. Each of these categories represents a different type of action taken by users in relation to a specific venue. 


\section{Place-naming}

Foursquare's venues database infrastructure allows users to create their own venues without any naming guidelines. Namely, since Foursquare's venues database is entirely based on user-created places, users can freely create and name any kind of venue they wish and add it to the growing database. Past studies showed that users who were able to create their own venue names used that to list names that made sense mainly to the people in their group (Barkhuus et al. 2008). This concept was termed "private geographies," a mutual sense of different places that differentiates social groups (Brown et al. 2007).

Seventeen out of the twenty-five users I interviewed confirmed that they had created at least one venue since they first started using the application. Usually, they created these venues when a new business opened and it did not appear on the list. In addition, they also utilized this function in case they decided to open venues that served as an inside joke among their group of friends (such as "hell"). Although creating fake venues is a common practice, it is perceived as controversial. While several users saw it as a way to create interest in Foursquare, others saw it as a gateway to create noise and weaken Foursquare's network. As Michael, ${ }^{3}$ twenty-six, a system analyst from Pittsburgh describes:

There have been several bars and such in the neighborhood that I created a location for.... I think it's a slippery slope though. I get annoyed when I see people add their house $e^{4}$ or some made up location to the list. I am not part of it and it feels like it's diluting the system.

As we can see, Michael does not like seeing made-up venues in his app. In a way, these places represent a local surrounding that he is not part of and therefore he feels he is alienated. He would rather prefer to see all the venues he knows and has connection to in his neighborhood. Similarly, John, thirty-one, a graphic designer from New York City, explains his logic behind creating venues on Foursquare:

I don't create locations unless I am connected to them.... I prefer to be a tastemaker in my habits on Foursquare, to give constructive tips that would be helpful to someone and inspire them to visit.

John's comment offers us a way to better understand the venue creation and labeling process as one that embeds the user's personal connection and history in it. Each venue created by John reflects his personal taste, his past interactions with and within the place and also with the people there. In this way, John utilizes the application to spread his local knowledge and promote others to visit and experience the places he likes most. Moreover, John uses the tips options provided by the application to leave notes of his experiences in the places he visits. By adding tips and photos to check-ins, users like John augment their 
check-in action and supplement additional information layers to it. These layers of content include photos taken at certain places and automatically uploaded, as well as a textual layer that includes the tips, likes, and lists in which the place appears.

In other words, when a user checks-in to a local bake shop in their neighborhood, they see not only the tips that other users left there but also pictures, the number of lists this place appears in, and personal information such as the last time they visited there, which of their friends was also there, how many times they have been there this week, etc. As a result, users' check-in actions create large metadata that are added to the different venues, data that are constructed of both visual and textual information based on people's history of visitation.

\section{Ownership}

When a user visits and checks in to a certain place frequently, they will ultimately receive the mayor title of that venue. The title itself sometimes offers rewards in chain stores, bars, etc. but normally it is deprived of any physical meaning. Despite that, the users I interviewed who got to be mayors felt a special connection to the place of their mayorship. As Sean, twenty-five, a computer programmer from Pittsburgh, puts it:

I know that the baristas at Starbucks are trained to interact with their customers so I knew them before but then being the Foursquare mayor of the place, it is ridiculous, but it still adds a bit of a status to the relationship I have with the venue and the people in it. So we joke about that a lot that I am the Foursquare mayor and any time there is a new employee I'm like "yeah, I am the Foursquare mayor." So it is something that does, although it has no meaning, set me apart from everyone else.

As we can see, the feeling of ownership and the status of the title creates a unique tie between the user and the place - a link that separates them from everyone else. As Sean points out, his mayorship distinguishes him from the general public that frequents this Starbucks. Everyone should know he is the mayor and as he notes his connection to the place and the people that work and socialize there is stronger. For another example, take Patrick, thirty-four, an artist from San Francisco, who describes his feelings about being the mayor of the local bar:

I feel like I have some type of ownership to that mayorship. I probably go there more than most people. It may simply be because my boyfriend works there and it's the closest bar to my house but I still got a little pissed when someone took my mayorship there for like a day. I reclaimed it quickly. I didn't know who this new mayor was and I didn't feel like he deserved to be mayor. 
As Patrick shows us, being the Foursquare mayor of that specific bar promoted him to take special action when he felt the place was falling to the wrong mayorship hands. His special connection to the place made him go out and reclaim the title, as the new mayor was not deserving of it.

The game mechanics embedded in the service often lead to "mayorship battles" between avid users (Oliver 2010). The political mess that is Foursquare mayorships scaled to completely new levels in the case of the Delicatessen, a trendy restaurant on the corner of Lafayette and Prince Streets in New York City. On July 14, 2010, Jennifer Magnolfi checked in to the restaurant and used the "shout" option of the application to call for the dethroning of her fellow Foursquare user, Baratunde Thurston, from his current mayorship of the place. The mayor was not amused by the challenge, as he put it:

I was aghast at the boldness, rudeness and crassness of the move. It was literally a slap in the face, insofar as iPhone pop-up notifications can be interpreted as physical assaults.

(Covington 2011)

The duo decided to take the challenge and compete over the mayorship during a period of thirty days. In this time frame, if Jennifer became mayor, she would be the entitled mayor. During this month, they both ran a real political campaign, with live-tweeting, blogging, support rallies and endorsements from the restaurant's owners. In addition, the restaurant itself created a cocktail for both competitors and hosted a victory party on August 16 for the winner.

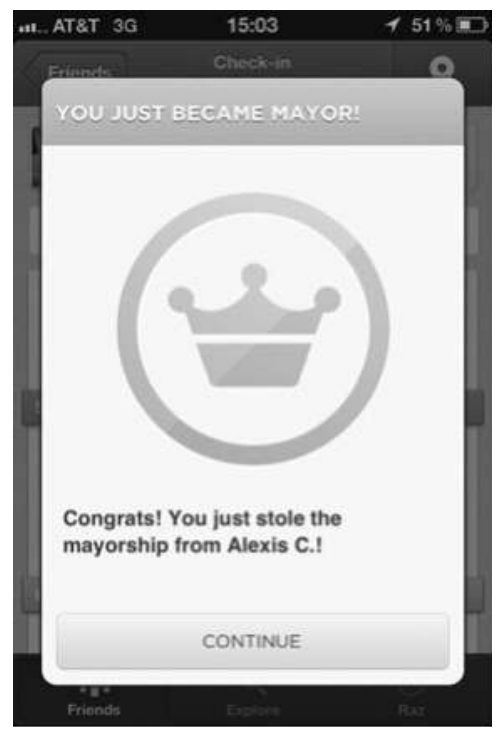

Figure 5.2 Mayorship change message (C 20I4, Foursquare Labs, Inc). 
At the end, due to Baratunde's business trip, Jennifer claimed the mayor title as she checked-in more times than he did. Shortly after, Baratunde gave his concession speech and congratulated Jennifer for her achievement (see Figure 5.2).

This example shows us how the social interactions created by the application and more specifically, the mayorship game mechanism, provides its users with ways to interact with the place as well as to display their strong feelings toward it. The campaign not only included the people that visit and work at the restaurant, but it was also covered by the press and presented later on in several social media conferences (Covington 2011).

\section{Events}

In addition to mayorships, a big part of the Foursquare game mechanics includes the badges system. Using the application in various scenarios rewards the user with virtual badges. For example, if a user takes ten trips to the gym in thirty days, she will receive the "Gym Rat" badge. If a user checks in at twenty different pizza places, she will be rewarded with the "Pizzaiolo" badge.

Some badges are harder to get than others. One of the more sought-after badges is the "Swarm" badge. A virtual medal saying that a user checked in to a place with more than fifty other people checked in there at the same time. As Foursquare adoption rate grew, it became easy to get it in the big cities, and users started planning special gatherings to unlock the "Super Swarm" badge that requires 250 people checked-in at the same time.

One of the first special "Super Swarm" badge parties took place on Thursday, October 7, 2010, at the Jewel Bar in London. A group of avid British Foursquare users decided to plan and hold a special event meant to unlock this special badge. The word about the festivity was spread around through Facebook, Twitter, and blog postings that encouraged users to join the organizers at the bar on the chosen date between 6P.M. and 9P.M. The event was a huge success and, as a result, the badge was unlocked and was indeed awarded to the cheering and celebrating group at 7:08 P.M. Bottles of champagne, courtesy of the bar, were then popped open and the celebrations kept going for several more hours.

As a result of the event, many users uploaded their pictures and shared them among themselves and the group activity was later extended to future events that also took place in the Jewel bar, all documented through users' check-ins, blog posts and photo galleries on Flickr which served as memorabilia of the event. ${ }^{5}$

Thirteen of my twenty-five informants remembered exactly when and where they got their first swarm badge. For example, Simon, twenty-five, a graduate student from Pittsburgh, noted:

I got the swarm badge when I was at a Pirates game at PNC Park. I was one among the first to check in there and I had to keep logging back in to see if it got over 50 and by the time it was over 50, my check-in already expired 
so I had to check in again to receive the badge. When I got the badge I showed all my friends there I got the badge. I looked through all the people that checked in there but I didn't recognize any of them.

Just like for Simon, for many of my interviewees, getting this badge was part of attending a music or sport venue but for several it was during special meetings such as 4SQDay celebrations. ${ }^{6}$ These events create a special meeting venue for avid users of the service to celebrate their joint enthusiasm for the application and in many of these gatherings the users receive special badges.

\section{Discussion}

Place-naming, ownership, and events serve as ways in which Foursquare users interact and explore their ties to the physical places they visit. To better understand these interactions I suggest applying the theoretical framework of "Place Attachment" by which we can study the factors that connect people to places and how they come about in our daily life. More specifically, I focus on three of the factors Low (1992: 166) identified in her work in relation to their representation in Foursquare interactions. These three factors include the vehicles of storytelling and place-naming, ownership, and, finally, celebratory events. As we can see from the results, each one of these ideas is articulated in social practices that combine both online and offline activity.

First, practices such as creating venues, leaving tips, and formulating lists adhere to the place-naming factor identified by Place Attachment theory and therefore can be regarded as a tool for the creation of a more elaborated narrative of the physical place. As labeling or tagging the places we visit becomes a common practice, location-based services like Foursquare promote users to create new relationships as well as maintain personal connections to the places they visit. Second, as Low notices, ownership of a place creates a strong attachment to it. In Foursquare we see that by negotiating in political acts such as mayorship battles, its users create and maintain an emotional relationship both with the physical place and the people who visit it, be they friends, strangers, or familiar strangers (Schwartz 2013). ${ }^{7}$ Finally, taking part in events such as swarm badge and 4SQDay parties, participants are encouraged to expand their local social connections as well as have the venue itself take a central role in their memory as the place of the festivity.

Following these findings, I argue that these type of interactions and the resulting personal attachment they create can be best described as "Online Place Attachment," online-offline interactions that carry the same characteristics that create people's connection to places, but at the same time represent sets of factors that result from their simultaneous online existence: extensive documentation, collective attachment, and mutual connection.

Online place attachment is paired with extensive documentation of the places we visit. Location-based services like Foursquare encourage us to document our 
daily lives. In this way, the actual action of checking in to a place is documented but it is also accompanied in many instances by other metadata such as photographs and tips that result in a rich documentation of our daily interactions. One might call it a documentation of our relationship with the places with which we have special ties. This kind of detailed documentation was indeed present before (like in the documentations made by the flâneur, or the psychogeography drawings of the city by the Situationist International movement members) but not in the scale we are seeing today.

Online place attachment is also tending to promote a collective attachment to a place as the interactions that take place on Foursquare are automatically shared with the user's friends. More specifically, when we check in to a location, all my friends that use the application see my check-in, and they can comment and "like" my action. Consequently, other users are becoming aware of the places I frequent and my experiences there.

Many studies consider the length of residence in a place to be an important factor in the creation of place attachment (Kasarda and Janowitz 1974; Ahlbrandt 1984; Taylor 1996). Our personal connection to a physical place is built over a period of time and through reoccurring visits. This factor is similarly important in the use of Foursquare as mayorships or badges are awarded only to users who regularly frequent the places. But still, it seems that the usage of the application creates a more rapid connection to the place since we can interact with the place not only when we are there but also later when we see our friends check in there, when we add it to a favorite places list, or when we are reminded by the application that we are near the place and we should check in there.

The "Radar" option on Foursquare is a fairly new feature that was made to encourage users to open up the application and check in to places they frequent. This programmed reminder can also be read as the representation of the place as an entity. Foursquare acts on behalf of the place to remind us of our relationship and that we should maintain it by checking in there. As a result, the place itself has its own wishes in our mutual connection.

It is however important to be aware of the interactions that do not play out in these online circumstances and both the users and places that are missing from the game. In other words, due to the demographics of geosocial applications (a higher percentage of Hispanic than whites or blacks, and of suburban adults than rural adults ${ }^{8}$ ), the interactions that take place over the application can be seen as promoting and strengthening the ties of only specific type of places that are frequented by a specific demographic that uses the platform. The affordance of the technology limits this type of Online Place Attachment to only a small group of people who are technologically savvy and who are usually located in urban areas, resulting in social exclusion.

Although this kind of interaction is still in its primary stages, I identify Online Place Attachment as a technological way to reinforce and maintain our relationship with the places we visit both through online and offline interactions. This form of attachment uses the same ways we know from previous forms of place 
attachment, which listed the offline experiences of individuals, but also adds to it the specific characteristics of online connectivity with all of the abovementioned naturally derived special characteristics. In this way, our use of the application reorders our daily life and affects our definition of relationships with physical places.

\section{Conclusion}

As the results show us, @IAmAru's emotional tweet is hardly a rare example; it portrays a growing tendency of intimate connection between people and places mediated by location-based technology. More specifically, the spread of mobile devices and the growing adoption of location-based social networks such as Foursquare play a significant role in the way we conceptualize and interact with the places around us. These special places are now invested with both our online and offline personal memories and therefore play a significant role in both our local identity as well as the shaping of our local interactions.

This work contributes to the existing literature by expanding the tool kit of theoretical frameworks we can use when we examine location-based technology, as well as offering a new term to identify the hybrid online-offline tie to physical places. Applying existing theoretical frameworks such as Place Attachment to the study of location-based technology offers a new lens through which we can articulate the ways these services may contribute to the formation, maintenance, and preservation of the interactions between a person or a group of people with the physical place.

I utilized this theory in order to study how Foursquare allows its users to create personal attachment to a specific venue. More specifically, I showed how Foursquare users' actions align with earlier identified practices of place-naming, ownership, and celebratory events.

Further research should examine how Online Place Attachment is materialized on other technological platforms and the significance this kind of attachment has over the activity of local social groups such as urban neighborhoods. Moreover, the study of the places that do not appear on the venue list as well as the most checked-in venues could provide meaningful insights to the study of the nature of places that maintain Online Place Attachment interactions with their patrons.

Studying these emerging virtual-local relationships of people and places in light of Online Place Attachment enables us to better understand users' actions and explore how, in turn, these practices promote the assimilation and participation of users in their local community, enhance relations with other users, and fortify the existence of our local identity.

\section{Notes}

1 https://twitter.com/IAmAru/statuses/27539654790.

2 https://foursquare.com/about. 
3 All the participants' names that appear in this work are fictitious in order to conceal the users' identity. This study is approved with IRB number HS11663.

4 In September 2011, mostly due to the launch of websites like www.pleaserobme.com that warned users to the dangers of disclosing their home location, Foursquare changed their policy in regard to check-ins to private homes. As a result, only the person who created the "home" location and their friends can now see the home address on the app. Venues that are categorized as "homes" only show up in the "nearby places" list if a user is friends with the creator of the venue. The original blogpost at: http://blog.foursquare. com/2011/09/29/making-your-house-into-a-home/ has been taken down, but it is preserved in part on Facebook: www.facebook.com/foursquare/posts/287135594636079.

5 Photo Gallery available at: http://londonist.com/2010/09/foursquare_londonist_ cohosts_event.php; superswarm.posterous.com.

6 4SQDAY (Foursquare day), a numerical pun, celebrated on April 16 of every year, was conceived in 2010 by Nate Bonilla-Warford, an optometrist from Tampa. Nate circulated the idea on Foursquare's Getsatisfaction.com forum and as a result he started getting help and support from many other Foursquare users who felt they wanted to take part in this celebration. Word spread via Twitter and Facebook and eventually Foursquare joined the flock and officially announced it on March 26. Since then, the day has continued to be celebrated, and in 2011 there were more than 1,200 Foursquare events around the world. Nineteen mayors declared official 4SQDay in their cities and Foursquare had more than three million check-ins in one day.

7 This type of territorialization work through location-based technology can also be seen in previous research of Japanese mobile gaming (Licoppe and Inada 2008).

8 According to Pew Internet Research Report: www.pewinternet.org/Reports/2013/ Location/Main-Report/LocationBased-Services.aspx.

\section{References}

Ahlbrandt, R. S., Jr. (1984) Neighborhoods, People and Community, New York, NY: Plenum Press.

Augé, M. (1995) Non-Places: Introduction to an Anthropology of Supermodernity, London: Verso.

Barkhuus, L., B. Brown, M. Bell, M. Hall, S. Sherwood, and M. Chalmers (2008) From awareness to repartee: Sharing location within social groups, in Proc. of CHI, Florence, Italy, pp. 497-506.

Brown, B., D. D. Perkins, and G. Brown (2003) Place attachment in a revitalizing neighborhood: Individual and block levels of analysis, Journal of Environmental Psychology, 23(3): 259-271.

Brown, B., A. S. Taylor, S. Izadi, A. Sellen, J. Kaye, and R. Eardley (2007) Locating family values: A field trial of the Whereabouts Clock, in Proceedings of UbiComp 2007, Innsbruck, Austria, Springer, pp. 354-371.

Campbell, S. W. and Y. J. Park (2008) Social implications of mobile telephony: The rise of personal communication society, Sociology Compass, 2(2): 371-387.

Covington, L. (2011) Foursquare File: Baratunde Thurston, Metromix.com, formerly available at: http://newyork.metromix.com/restaurants/article/foursquare-file-baratundethurston/2095336/content.

Cranshaw, J., R. Schwartz, J. I. Hong, and N. Sadeh (2012) The Livehoods Project: Utilizing social media to understand the dynamics of a city, in Proc. International Conference on Weblogs and Social Media, Dublin, Ireland. 
de Souza e Silva, A. (2013) Location-aware mobile technologies: Historical, social, and spatial approaches, Mobile Media and Communication, 1(1): 116-121.

de Souza e Silva, A. and J. Frith (2010) Locative mobile social networks: Mapping communication and location in urban spaces, Mobilities, 5(4): 485-506.

de Souza e Silva, A. and J. Frith (2012) Mobile Interfaces in Public Spaces: Locational Privacy, Control and Urban Sociability, New York, NY: Routledge.

de Souza e Silva, A. and L. Hjorth (2009) Urban spaces as playful spaces: A historical approach to mobile urban games, Simulation \& Gaming, 40(5): 602-625.

Dourish, P. (2006) Re-space-ing place: Place and space ten years on, in Proc. ACM Conf. Computer-Supported Cooperative Work CSCW 2006, Banff, Alberta.

Farman, F. (2011) Mobile Interface Theory: Embodied Space and Locative Media, Abingdon/New York, NY: Routledge.

Gordon, E. and A. de Souza e Silva (2011) Net Locality: Why Location Matters in a Networked World, Malden, MA: Wiley-Blackwell.

Humphreys, L. (2008) Mobile social networks and social practice: A case study of Dodgeball, Journal of Computer-Mediated Communication, 13(1): 341-360.

Humphreys, L. (2010) Mobile social networks and urban public space, New Media \& Society, 12(5): 763-778.

Kasarda, J. D. and M. Janowitz (1974) Community attachment in mass society, American Sociological Review, 39(3): 328-339.

Licoppe, C. and Y. Inada (2008) Geolocalized technologies, location-aware communities and personal territories: The Mogi Case, Journal of Urban Technology, 15(3): 5-24.

Ling, R. (2004) The Mobile Connection: The Cell Phone's Impact on Society, San Francisco, CA: Morgan Kaufmann.

Low, S. M. (1992) Symbolic ties that bind: Place attachment in the plaza, in I. Altman and S. M. Low (eds.), Human Behavior and Environments: Advances in Theory and Research. Volume 12: Place Attachment, New York: Plenum Press, pp. 165-185.

Low, S. M. (2000) On the Plaza: The Politics of Public Space and Culture, Austin, TX: University of Texas Press.

Low, S. M. and I. Altman (1992) Place attachment: A conceptual inquiry, in I. Altman and S. M. Low (eds.), Human Behavior and Environments: Advances in Theory and Research. Volume 12: Place Attachment, New York, NY: Plenum Press, pp. 1-12.

Manzo, L. C. and D. D. Perkins (2006) Finding common ground: The importance of place attachment to community participation and planning, Journal of Planning Literature, 20(4): 335-350.

Milligan, M. J. (1998) Interactional past and potential: The social construction of place attachment, Symbolic Interaction, 21(1): 1-33.

Oliver, S. S. (2010) Who elected me mayor? I did, New York Times, August 19, available at: www.nytimes.com/2010/08/19/fashion/19foursquare.html? pagewanted=all.

Rainie, L. and B. Wellman (2012) Networked: The New Social Operating System, Cambridge, MA: MIT Press.

Riger, S. and P. J. Lavrakas (1981) Community ties: Patterns of attachment and social interactions in urban neighborhoods, American Journal of Community Psychology, 9(1): 55-66.

Schwartz, R. (2013) The networked familiar stranger: An aspect of virtual and local urban anonymity, in K. Cumiskey and L. Hjorth (eds.), Seamlessly Mobile? Mobile Media Practices, Presence and Politics, Abingdon/New York, NY: Routledge, pp. 135-149. 
Sheller, M. (2004) Mobile publics: Beyond the network perspective, Environment and Planning D: Society and Space, 22(1): 39-52.

Sutko, D. M. and A. de Souza e Silva (2011) Location-aware mobile media and urban sociability, New Media \& Society, 13(5): 807-823.

Taylor, R. B. (1996) Neighborhood responses to disorder and local attachments: The systemic model of attachment, social disorganization, and neighborhood use value, Sociological Forum, 11(1): 41-74.

Weiss, R. S. (1994) Learning From Strangers: The Art and Method of Qualitative Interview Studies, New York, NY: The Free Press.

Wellman, B. (2001) Physical place and cyberplace: The rise of personalized networking, International Journal of Urban and Regional Research, 25(2): 227-252. 


\title{
Chapter 6
}

\section{Location as a sense of place Everyday life, mobile, and spatial practices in urban spaces}

\author{
Didem Özkul
}

Physical mobility and information and communication technologies (ICTs) have altered the perception of space and time. They influence the way we perceive distances as shrinking (Harvey 1989) or increasing (Giddens 1990), by providing the users of these technologies with a possibility of communication while on the move. Although the use of ICTs may lead to a false perception of location and distance as less important, "distance still matters a lot to people, as does place" (Bærenholdt and Granås 2008: 7). Mobile communication technologies foster attachment to places by creating a renewed interest in location. Networking and mobile technologies can "only contribute to material, social and cultural reconfigurations of places and distances" (ibid., my italics) and therefore they have the potential to influence what a place represents and embodies for its inhabitants.

Research on mobile communication technologies has focused on changes in social and spatial practices of everyday life (Goggin and Hjorth 2009; Ling and Campbell 2009). Mobile technologies are questioned concerning the large extent to which they blur the lines between public and private space, work and personal life, and coordinate for social networking (Ling and Campbell 2009; Lee 2009; Humphreys 2008). With locative media, the focus of mobile media research has shifted toward the analysis of location-based applications and their use in everyday life (de Souza e Silva and Sutko 2009; Humphreys 2007; Humphreys and Liao 2011; Licoppe and Inada 2009). Although recent scholarly works explain locative media use in relation to theories of space (Gordon and de Souza e Silva 2011; Wilken and Goggin 2012), further empirical study is needed to explore how people actually use locational information in everyday life.

Thinking of this gap in the mobile and locative media literature, I conducted thirty in-depth interviews with smartphones users in London during the year 2011. ${ }^{1}$ London's inhabitants are cosmopolitan in nature, and maintain a complex network of social ties, both near and distant, which are maintained by using mobile phones. Hence, London is an ideal site for researching different aspects of place-making through mobile and locative media. This chapter is an analysis of interesting aspects of place-making and place attachment in London, aiming to explore the connections between locational information use on smartphones 
and reconfigurations of place. By grounding empirical research in recent frameworks and classical theories of place and location, this chapter also aims to further the understanding of location and use of locational information in mobile communication.

Location has been conceptualized as an aspect of place (Lukerman 1964; Relph 1976), and an important attribute of many practices in everyday life. Sharing the location of any place does not turn places into locations, nor can locations simply be turned into places (de Souza e Silva and Frith 2012). Sharing locational data can only change the sense of a place, how we perceive it and what a place means to us. When users of mobile and locative media check in at a place or attach location information to places, they actually share their memories and understandings of those places, along with the physical coordinates of those places.

People retrieve or disclose locational information in everyday life for many reasons: To deal with the anxiety of getting lost, organize daily activities and be punctual, gain a feeling of security, discover new places, establish social relations, and maintain close ties, among other things. These kinds of activities form the basis of this study in answering two research questions: (1) How do people identify their physical locations within their daily activities? And (2) In what ways do they refer to, or evoke, place while using mobile ICTs? The findings of this study discuss and demonstrate how locational information use can renew senses of places and reconfigure spatial practices.

\section{Literature review}

Mobility in everyday life can lead to false perceptions of decreasing importance of place and location. This is due to a general representation of mobility as opposite to place (Simonsen 2008). Mobility is usually associated with detachment from places, placelessness or with having "no sense of place" (Meyrowitz 1985; Relph 1976) as "mobility has been associated with a lack of connection and commitment" (Larsen and Urry 2008: 92). Mobility (and mediation) has been blamed for leading to an accelerating erosion of place (Augé 1995) and the hypermobility of flexible capital, mass communications, and transportation are seen as threats to place (Cresswell 2002).

The popularity of communication technologies has often also contributed to the feelings that distance and location information would lose their importance and detach us from place because they trigger physical mobility. As Meyrowitz (2005: 27-28) states, "travel is more easily managed as distant places seem less strange and less dangerous and as contacts with those 'back home' (or anywhere) can be maintained wherever we roam." As argued by Simonsen (2008), increased mobility, information technologies, and consumer society, combined, have been blamed for accelerating erosion of places: "More and more of our lives, it is said, take place in environments that could be anywhere-that look, feel, sound, and smell the same wherever in the world we might be" (2008: 13). 
However, attachment to places always exists no matter how mobile we become (Gustafson 2002; Relph 1976), because mobility can also be understood as "a way of finding meaning and ways to places and belonging" (Bærenholdt and Granås 2008: 6-7). Mobile communication increases our chances to attach to new places, while helping us maintain old attachments. On the other hand, it allows its users to detach from places and co-present situations willingly in order to experience different aspects of the spatial environment (Gordon and de Souza e Silva 2011; Humphreys 2007; Ozkul and Gauntlett 2014). Therefore, detachment from a place should not be understood as a negative aspect of mobile communication because it is not the same thing as erosion of place.

Today, cities contain information from various networks, of both people and devices, and what we now perceive about a place is actually well beyond what is physically in front of us (Gordon and de Souza e Silva 2011). Through mobile communication technologies such as the smartphone, users can assign meanings to places by attaching geotagged information to locations which others in turn can access while at the location (de Souza e Silva and Frith 2012). This enhances the awareness of multiple meanings of places, as users can explore many aspects of a city that are not explicitly there in its visible physical fabric. In some cases, this use of location information may allow users of mobile communication technologies to create and share their own genuine experiences of places (Ozkul and Gauntlett 2014) and to bring different senses of places into conversation (Humphreys 2007), creating new forms of attachment to places. Therefore, mobile communication technologies, in general, and locative media, in particular, do not only cause a feeling of detachment from places; they simultaneously afford and renew attachment to places as part of being mobile.

Although the definition of location is usually subordinated to the notion of place, location and place are not the same thing (Cresswell 2004). Location is more specific than place (Relph 1976). "Place is made up of a number of things that can be specifically located" (May cited Relph 1976: 4). In everyday language we usually use the word place to refer to a location (Cresswell 2004). However, "a place is not just the 'where' of something; it is the location plus everything that occupies that location seen as an integrated and meaningful phenomenon" (Relph 1976: 3).

A meaningful location can also be understood by its sense of place, since it means "the subjective and emotional attachment people have to place" (Agnew cited Cresswell 2004: 7). Place attachment is a multi-faceted concept and defined as "bonding that occurs between individuals and their meaningful environments" (Scannell and Gifford 2010: 1). Central to the concept of place attachment are affect, emotion, and feeling (Altman and Low 1992). For some scholars, place attachment is a sub-concept of sense of place (Scannell and Gifford 2010). Sense of place is usually associated with the identity of a place and people's selfidentifications with and within that place (Nairn cited Relph 1976). It is not only about a positive/negative feeling for a place, but also derived from the totality of one's individual life (Eyles 1985). It lies within our existential relationship to the 
world (Simonsen 2008: 14) and can take various forms based on individual perception. Relph (1976: 63) discussed that "the most meager meaning of "sense of place' is the ability to recognize different places and different identities of place." Identity of a place provides an individuality to a place in comparison to other places as separable entities and allows a specific place to gain some distinction (Lynch 1960). Since the location of a particular place is distinct from that of others, locational information can be one element that is used to identify different places.

Location and mobility play a crucial role in constructing and reconstructing senses of place. Places are mobile (Langer 1953; Sheller and Urry 2006), but location is usually conceptualized as "neither a necessary, nor a sufficient condition of place" (Relph 1976: 29). In agreement with Relph, Cresswell (2004) argues that places are meaningful locations. This line of thought degrades the importance of locational information in place-making while prioritizing other aspects of place attachment, such as cultural and emotional meanings. On the other hand, with mobile and locative media use, location became more discernable and important as a feature of place. As the sharing of locational information started to acquire dynamic meanings with the use of mobile and locative media (de Souza e Silva and Frith 2012), statements or markers of location began to contribute to the sense of a place. As a result, places acquire different meanings, not only for the ones who share locational information but also for those who receive it. Hence locational information is an important attribute of a place, influencing place-making and our understanding of places.

\section{Methods}

This chapter seeks first to understand the various forms that a "sense of place" might take for different individuals, and second to explore how the sharing of locational information using mobile devices contributes to constructing different senses of places, especially in highly mobile cities such as London. It also aims to develop an understanding of locational information sharing, which can also be affected by feelings of belonging and attachment to places. For this purpose, I conducted semi-structured in-depth interviews with thirty smartphone users, in London, of whom 40 percent were male and 60 percent were female. I used Twitter and snowballing to recruit participants. The random sample of thirty volunteers were aged between nineteen and fifty-eight. Therefore, it was not my aim to have a statistically representative number of smartphone users in London, but to understand different, everyday uses of smartphones in order to design a larger study.

London is the most populous city in the EU (London Councils 2013), and can sometimes be overwhelming and hectic. The total number of passengers who ride on London underground transportation alone is 1,107 million (ibid.). This, combined with the cosmopolitan nature of its inhabitants, makes London a rich ecosystem for understanding different constellations of place, location, and 
mobile phone use. Hence, the importance of locational information in navigating such a complex city cannot be underestimated.

I chose smartphone users as the so-called feature phones do not generally involve much use of locational data. Additionally, today, especially in industrialized societies, mobile phone subscribers are unintentionally using a location determination technology (Steinfield cited in Fraunholz et al. 2005: 132-133), because users have no other choice than to buy phones that have location-aware features (Sutko and de Souza e Silva 2011).

I started the interviews with general questions about smartphones such as the reasons for use and types of applications used. I avoided directing questions about location-awareness and instead waited for the respondents to start talking about location-based applications. My plan was to limit the understanding of locational information only to location-based applications. However, during the interviews respondents also referred to Facebook status updates, phone calls, or photos when they were talking about locational information. Therefore, locational information, in this study, does not solely consist of location-based services, but also textual and visual location representation. After transcribing the interviews, I used content analysis in order to find repetitive themes. Several factors were found to be influential on the relationship between locational information use and sense of place.

\section{Findings}

Based on how participants used locational information on smartphones in London, two themes emerged: navigation and place attachment.

\section{Navigation: finding and getting lost}

One of the major uses of locational information was to locate oneself on a map and navigate in London. Being able to locate oneself in a foreign place can contribute to the feeling of having local knowledge of a place. Using the map features on their smartphones, participants stated that they felt secure, empowered, and local, since they knew that they would never get lost as long as they have their phones with them. Smartphones become more important for navigating in the city, especially because of the commuting culture in London. As one of the respondents explained during an interview:

People commuting in London do learn quickly that you don't ask questions.... If you're asking somebody a direction while commuting, that culturally almost causes somebody a tension.

$(\text { Tina, } 32)^{2}$

Another respondent also stated that asking people directions in London was not the best solution when you are trying to find a place: 
I don't like asking people all the time. People in London don't know where to send you anyway, because nobody is from this place. So whenever you ask somebody in London "Where is this street?" they have no idea.

(Mark, 32)

Navigational location information creates a feeling of belonging and local knowhow as it diminishes the anxiety of getting lost. When Mary first got lost in London, she didn't have a smartphone. She used her mobile phone to call her husband:

When I first came to London, I got lost and I called Tim. He said "Look for the BT Tower!" I was crying and I felt so disempowered. And now I don't. I want to navigate through the space myself, because then I get empowered.

(Mary, 35)

When participants can navigate in the city using the locational information on smartphones, the city looks familiar and newcomers can feel as if they are local. However, even locals do not always know every part of London. As Tina explained, London can sometimes be hard to navigate:

London is a large place. You may not know the differences between North and South London. My friends from North London never come to South London, so they wouldn't know how to get around South London.

(Tina, 32)

On the other hand, relying solely on maps on smartphones can sometimes lead to anxiety itself because of inaccuracy. Another participant was going to a friend's party. On the bus he used his smartphone to check location:

It doesn't update very quickly, so I missed the bus stop. I thought "Oh, I am still here, it is not time to get off yet," but then the bus moved and the blue dot suddenly jumped there ... I thought that it was my stop, but the map said something else ... I trusted the application instead of the bus!

(John, 27)

Although maps on smartphones can have problems with accuracy, people with a bad sense of direction find them very helpful in making sense of new places. For example, Amy explained how she used maps on her phone.

You know the blue bubble, it is clear, it moves with you. So I think it is helpful. It also gives me a sense of the place and how I am moving because I am not really good at reading maps otherwise. 
It is sometimes more important to know one's whereabouts than having a direction which tells you when to turn right and left. Since maps on smartphones provide the users with familiarity - and familiarity is a cognitive component of place attachment (Fullilove 1996) - they can contribute to place attachment and establish a sense of a new place. Discussing the role of familiarity in place attachment, as Scannell and Gifford (2010: 3) argue, "to be attached is to know and organize the details of the environment."

Converse to the idea of using locational data in smartphones to find specific places, it can also be used to get lost willingly and explore new aspects of urban spaces, generating a new sense of places (or in other words, a new way of placeing oneself). One of the motivations to use locational information as such is to explore new places and escape from the monotony of everyday life. Participants mostly stated that they use various applications, such as Serendipitor and WikiMe, as well as sometimes running Google Maps, to generate a random route (and hoping to see new places and experience new things).

They sometimes prefer using those random or recommended routes by others instead of their everyday routines. So they use locational information as a means of getting lost and detaching oneself from the ordinariness of everyday life. With these means they can also create (re)attachment to (old) places by discovering new things in that environment. Using a new and different route may in itself be enjoyable and exciting. One of the participants indicated that he used his location information to explore new things and added: "Will I find something interesting?" When talking about Serendipitor, he described it as "an artistic project which adds aesthetic dimensions to the city" (Ben, thirty-two). When I asked him if there was a difference in experiencing the city using those apps or just by wandering in a city without a smartphone, he said:

There is a difference because the agency as such is in the actual gadget (pointing his smartphone). Of course it is different.... Because something is driving me ... I'm not driven by my will, it is just an algorithm guiding me through the city.

Participants who revealed interest in applications such as Serendipitor ${ }^{3}$ believed that those applications blur the boundaries between physical and digital. In Ben's use of Serendipitor, although he experiences London at a physical level, using his smartphone to find "something interesting" he also digitally experiences aspects of London which may not be physically observable or available. Therefore, the users could discover and experience new things in the city through their mobile devices. Another respondent talked about how she experienced London by using an application called Hidden London, which is like a treasure hunt game for places:

It is blurring the boundaries. As I said, you are not anymore only in the street, or in the spaces as a being among other beings and buildings, etc. 
You are like in an application, in a program world, and you develop a different persona.... It is like "Oh, we're bored. Didem, where should we go? Oh, let's look!"

(Mary, 35)

On the other hand, using such applications to explore new things and places was not always desirable. For Andy (thirty-five), wandering in a city on his own was a better experience than following a predetermined route. He described such location-based applications as diminishing our "sense of the city as a living space." Because he thought that "it can never replace what is happening in the real world," for him those applications turn places into locations. Interestingly, later during the interview, Andy agreed that the application that keeps track of his running routes and shares it with other users actually helps him to plot new routes and try something different.

\section{Place attachment: identity, memory, and togetherness}

Individuals can draw similarities between themselves and places, and do connect to places which comes to represent who they are (Scannell and Gifford 2010). With the proliferation of locative media and especially location-based social networks "location has become an important piece of personal and spatial identity construction" (de Souza e Silva and Frith 2012: 163). By sharing locational information, especially through a social networking application or a locationbased game, users of mobile and locative media can show with what places they associate themselves. By controlling (what) to share and (what) not to share (about) a specific place, one does not only communicate different aspects of place-making, but can also present different aspects of the self.

Locational information use creating place attachment was mostly related to Foursquare check-ins. Paul (twenty-two) told me that to become the mayor of a place, he sometimes checked-in at places which could be mundane, such as a random pub. He could not explain why he engaged in such an interaction, which he described as an obsessive-compulsive behavior. He described how he became the mayor of the book shop where he used to work:

I worked there a day and a half every week. When I first found it on Foursquare, I sort of noticed that there was someone else, some customer, as the mayor ... "I work here, so this is mine. This is my territory! I've been here for three years. So I have this." So I made that a part of my empire.

(Paul, 22)

The user who checks-in more at a given place on Foursquare can become the mayor of that place, which can, symbolically, contribute to place attachment. So when one is ousted by someone else on Foursquare and loses a mayorship, the need of going back to that place and checking in to win that place back arises. 
Every now and then you get a little message saying you get ousted by someone. Then I say: "Old bugger! That's my place, I want that back!" And I don't know why I want it back!

On the other hand, respondents do not check in at places solely to become the mayor. One of the participants explained why she checked in at a boutique in Covent Garden. Her motivation was mainly to display and, in some sense, show off:

I went shopping the other weekend to Covent Garden, to Lily Allen's boutique. It was just a funny thing to tag in there and some of my friends from Wales, because I used to live in Wales, said "Oh my God! That is amazing!"

(Katy, 27)

Katy believed that she was using this information about that particular place to keep in touch with her friends, as she might have known who would comment on such a check-in. However, it reveals that she identified herself with Lily Allen's boutique, which was an ideal place for her to be. So she used it to show how she had been enjoying London, and in a way say: "Look where I am, and you are not!" Paul also used check-ins for a similar reason as Katy did:

Tell them particularly impressive things. Like say, "the British Library" and then I'm going to say a message "Look at me, I'm smart!"

In these circumstances, the meanings of places are shared in relation to personal identifications. They do not necessarily show one's affections or behavior toward a place, but they signify one's own traits and desired personal attributes. Therefore, location-based applications that use those maps help users attach to places either through self-presentation or presentation of different attributes of places.

Sharing "who am I" through locational information also includes recalling and recollecting memories in relation to places. By remembering, we can sometimes renew the meaning of places and the feelings they evoke, which strengthen our attachment to places. Riley (1979: 13) argues that "adult recall might show not a simple desire for the pleasure of place but a need for the nurture or support experienced there." Hence, "the remembering of a place may have less to do with the place per se, and more to do with yearning for the emotion or mood it once evoked" (Marcus 1992: 111). Conceived in this way, among other senses of places such as social and instrumental, nostalgic sense of place has a special importance for us (Ozkul and Gauntlett 2014).

As the respondents indicated, they usually navigate back to their photos, mobile Facebook status updates, and Foursquare check-ins to remember those places and recall memories (ibid.). One of the respondents told me that when he went to Spain, he wanted to check-in at a castle: 
These are the places that I don't want to forget and I tag them on Facebook as well.

(Jim, 22)

If the places visited are of high significance, users also share them on Facebook (mobile), add photos, and geotag them:

If it is very valuable ... if it's a very memorable place, then I go back there again as well.

(Jim, 22)

Also, as another respondent mentioned, users can sometimes add location information to their blogs via their smartphones to share their memories with their followers. Interestingly, they later search their own blogs to remember those places:

It is augmented memory. I search my own blog for things like that ... I like to blog and I like to share useful things around with people, but I also search my own blog to search for information on places that I have been before.

(Jason, 54)

Nostalgic feelings can be both positive and negative (Eyles 1985), hence the places that people check-in or share do not have to remind them of happy memories, but recalling them can evoke positive feelings about certain places. One of the respondents suffered from a rare disease and had lost her ability to walk two years ago. By the time of the interview she was using a wheelchair and had to go to medical centers almost every week. She used Foursquare, and even if her memories associated with the medical centers were not positive, she continuously checked in:

What amused me a lot was.... It (Foursquare) said to me that my most checked in places were medical centers and pubs! That made me laugh a lot. Because obviously I am always ill and I am always in the pub when I am not.

$(\mathrm{Liz}, 22)$

When she looked at her check-in history she was amused, not because of the places that she had checked in are amusing, but because she thought that Foursquare knew her quite well. Hence, nostalgia can be transformed into an amusing narrative, assigning a positive attribute to places. Memory is highly interpretive and it is not simply a replica or a duplicate of real-life events (Cattell and Climo 2002). Used as an interpretive tool, locational information sharing can renew the existing sense of places. 
As Meethan (2006: 4-5) argues, "places are invested in a variety of meanings that encompass notions of home, belonging, shared culture, shared language and history, and forms of personal and collective identity." Hence, in addition to being a means of remembering memories of places, people, or events on mobile social networks (Humphreys 2007), location information can also be used to create a sense of being together, again renewing the current sense of place where one is located. One of the respondents explained how she used her smartphone and location information explicitly (via photographs, rather than simply saying "I am here!") in order to feel together with her family.

My father is in India, my brother is in Ottawa, I'm in London, my sister is in Washington ... and sometimes you don't have the energy to talk on the phone, so it makes you feel a lot more connected!

(Becky, 22)

Feeling present and connected was very important as it was the major motivation for using location with photos. Becky continued to explain how she and her brother used their smartphones to create and maintain that feeling:

Yesterday, he did send me a photo of a very nice coffee, and I said "where is that?" because I didn't recognize it from the cup, or the setting, "I'm in Montréal!" I said "Wow!" Like when my father told me that "your brother is in Montréal," it is different. You feel like ... I'm kind of there, you are more part of it.

Participants used location information also to keep in touch with friends in London. London can sometimes be hard for people to meet and connect. For those circumstances respondents preferred to use Foursquare and Facebook Places where the former was more intended for such a purpose, while the latter was more intended to keep some kind of a diary. One of the respondents explained how check-ins could affect his connection with his family and friends.

You can't shout in a way ... "I'm at Paddington!" Well you can't do that. But virtually, you can tell your friends, you can tell your family, you can say "I am at this place!" Anyone might not see you physically at that place, but by saying you're virtually there they might come and say "Hi" (meeting in person), that's the good use of it!

(Jim, 22)

These kinds of locational information use on smartphones with or without location-based apps explain how people care for the loved ones, how they stay connected with each other whether or not they are living in the same city. In sum, how locational information creates a feeling of togetherness. 


\section{Discussion}

We usually think of notions of place and mobility as opposites. Physical mobility and ICTs, especially mobile technologies, had been seen as causes of erosion of place (Simonsen 2008). However, as many scholars from different areas agree, places can also be mobile (Cresswell 2004; Sheller and Urry 2006), and place and mobility are not opposites (Gustafson 2002). They should be thought of in relation to each other. In addition, although mobile communication technologies have provided its users with a detachment from place in the form of physical mobility, allowing their users to carry their connections with them, they simultaneously afford a form of attachment to places. No matter how mobile our everyday lives have become, we continue to value places, remember what they mean to us, identify ourselves with them, and communicate our identities through them.

Location is an important element of what constitutes a place (Lukerman 1964; Relph 1976); it is an aspect of sense of place. However, in daily language, the notion of place has been used to explain location (Cresswell 2004) and therefore location seemed to lose its significance in daily practices. Retrieving and sharing locational information can reconfigure our understanding of places. When location-aware technologies started to proliferate our everyday lives, we started to rethink its importance. Especially, when users of mobile and locative media check in at places, they do not usually think about the longitude and latitude of those places, but individual, social, material, and cultural values of those locations. Hence, what is shared is not the location per se, but what it evokes and reminds us of, the locational information.

The two categories of locational information use discussed in this chapter reveal the instrumental (as in the navigational location information use) as well as more inner, personal (as in the place attachment) aspects of place-making. Whether used in the form of retrieval (to discover new and different routes, find one's whereabouts, and establish spatial knowledge even by getting lost), or in the form of sharing (to leave traces of one's everyday life for future reference for one's self and others), locational information use can increase one's attachment to places. Hence, practices of retrieval and sharing of locational information can be brought together in order to understand location as a sense of place.

First, users of mobile technologies use navigational location information to find, create, or sense new places, empowering them with the feeling of having local knowledge of a new place. Additionally, by sharing their locational information, they can also establish a personal relationship with a place, and project what that place might mean for others when they see that information. Remembering feelings and emotions that a place used to generate, rather than the physical attributes of a place, can also contribute to place attachment. Used this way, either intentionally as a memory device (Humphreys 2007) or unintentionally (such as automatically geotagging mobile photos), sharing locational information can contribute to nostalgic feelings. As Cattell and Climo (2002: 1) argue, 
From the simplest everyday tasks to the most complicated, we all rely on memories to give meaning to our lives: to tell us who we are, what we need to do, how to do it, where we belong, and how to live with other people.

As a way of giving meaning to life, locational information could remind us of an event such as a gig that had taken place in that specific place, or people with whom one had been to that place. It not only evokes feelings for the user who shares the information, but also for the others who could access that information.

Therefore, mobile and locative media offer alternative ways for experiencing the city (de Souza e Silva and Frith 2012; Farman 2012). For some, it is believed to cause diminishing feelings of the sense of the city. However, locationawareness is not built to replace the real world. It only reconfigures our perception of place, adds another dimension to the experience of the city, sometimes contributing to new senses of places. Hence users of mobile and locative media can form attachment to places by feeling local and empowered while navigating in a city, remembering their memories related to the places they had been to and discovering new aspects of places that might had gone unnoticed within the fast pace of metropolitan life, as well as by sharing their own associations and experiences with places in a city. Exploring new things in a city is not only specific for users of those applications and those who share their locations. Nonusers who are somehow connected to those users via social networking can experience a new sense of a place based on their friends' locations.

\section{Conclusion}

The city expands, revealing the hidden and unnoticed, allowing the users of mobile communication technologies to explore and experience new aspects of spatial and social interactions with their physical environment. Every experience is unique, and locational information adds up to these experiences in a city. Contrary to the understanding of mobile communication technologies and mobile media as causes of erosion of place and diminishing meanings of places, location information use creates (re)attachment to places, allowing users to explore the hidden meanings and assign different ones to places. In order to understand how our perception of space and daily practices have changed with the use of mobile and locative media, as mobile media scholars, we should try to understand location and its use with mobile technologies rather than focusing only on specific location-based services (or applications) and behaviors such as checking in. There are other implicit ways of sharing location, such as sending photos in the form of a text message. Therefore a broader focus on the analysis of locationawareness can help us to better understand changes in the perception of space and the construction of the meanings of places. 


\section{Acknowledgments}

I owe special thanks to participants of this study for sharing their personal experiences. I also would like to thank Adriana de Souza e Silva and Mimi Sheller for their valuable comments and suggestions. Last but not least, I would like to thank to David Gauntlett and Lee Humphreys for making comments on an earlier version of this paper and making important contributions to it.

\section{Notes}

1 This study is part of Özkul's $\mathrm{PhD}$ research based at the University of Westminster.

2 Participants' names are changed in order to preserve their anonymity.

3 Serendipitor, by Mark Shepard, was developed at V2_ Institute for the Unstable Media as part of a joint artist residency with Eyebeam Art+Technology Center. It is an alternative navigation application for iPhone that help its users to find something by looking for something else (www.serendipitor.net).

\section{References}

Altman, I. and S. M. Low (1992) Place attachment: A conceptual inquiry, in I. Altman and S. M. Low (eds.), Place Attachment, New York, NY: Plenum Press, pp. 1-12.

Augé, M. (1995) Non-Places: Introduction to an Anthropology of Supermodernity, London: Verso.

Bærenholdt, J. O. and B. Granås (2008) Places and mobilities beyond the periphery, in J. O. Bærenholdt and B. Granås (eds.), Mobility and Place: Enacting Northern European Peripheries, Farnham: Ashgate, pp. 1-10.

Cattel, M. G. and J. J. Climo (2002) Meaning in social memory and history: Anthropological perspectives, in J. J. Climo and M. G. Cattel (eds.), Social Memory and History: Anthropological Perspectives, Walnut Creek, CA: AltaMira Press, pp. 1-23.

Cresswell, T. (2002) Theorizing place, in G. Verstraete and T. Cresswell (eds.), Mobilizing Place, Placing Mobility: The Politics of Representation in a Globalized World, New York, NY: Rodopi, pp. 11-31.

Cresswell, T. (2004) Place: A Short Introduction, Malden, MA: Blackwell.

de Souza e Silva, A. and J. Frith (2012) Mobile Interfaces in Public Spaces: Locational Privacy, Control, and Urban Sociability, New York, NY: Routledge.

de Souza e Silva, A. and D. M. Sutko (eds.) (2009) Digital Cityscapes: Merging Digital and Urban Playscapes, New York, NY: Peter Lang.

Eyles, J. (1985) Senses of Place, Warrington: Silverbrook Press.

Farman, J. (2012) Mobile Interface Theory: Embodied Space and Locative Media, New York, NY: Routledge.

Fraunholz, B., C. Unnithan, and J. Jung (2005) Tracking and tracing applications of 3G for SMEs, in M. Pagani (ed.), Mobile and Wireless Systems and Beyond 3G: Managing New Business Opportunities, London: IRM Press, pp. 130-154.

Fullilove, M. T. (1996) Psychiatric implications of displacement: contributions from the psychology of place, American Journal of Psychiatry, 153(12): 1516-1523.

Giddens, A. (1990) The Consequences of Modernity, Stanford, CA: Stanford University Press. 
Goggin, G. and L. Hjorth (eds.) (2009) Mobile Technologies: From Telecommunications to Media, New York, NY: Routledge.

Gordon, E. and A. de Souza e Silva (2011) Net Locality: Why Location Matters in a Networked World, London: Wiley-Blackwell.

Gustafson, P. (2002) Place, Place Attachment and Mobility: Three Sociological Studies, Göteborg: Göteborg University Press.

Harvey, D. (1989) The Condition of Postmodernity, Oxford: Blackwell.

Humphreys, L. (2007) Mobile social networks and social practice: A case study of Dodgeball, Journal of Computer-Mediated Communication, 13(1): 341-360.

Humphreys, L. (2008) Mobile devices and social networking, in M. Hartmann, P. Roessler, and J. R. Hoeflich (eds.), After the Mobile Phone: Social Changes and the Development of Mobile Communication, Berlin: Frank \& Timme, pp. 115-130.

Humphreys, L. and T. Liao (2011) Mobile geotagging: Reexamining our interactions with urban space, Journal of Computer-Mediated Communication, 16(3): 407-423.

Langer, S. (1953) Feeling and Form, New York, NY: Charles Scribner's Sons.

Larsen, J. and J. Urry (2008) Networking in mobile societies, in J. O. Bærenholdt and B. Granås (eds.), Mobility and Place: Enacting Northern European Peripheries, Farnham: Ashgate, pp. 89-101.

Lee, D. H. (2009) Re-imagining urban space: Mobility, connectivity, and a sense of place, in G. Goggin and L. Hjorth (eds.), Mobile Technologies: From Telecommunications to Media, New York, NY: Routledge, pp. 235-251.

Licoppe, C. and Y. Inada (2009) Mediated co-proximity and its dangers in a location-aware community: A case of stalking, in A. de Souza e Silva and D. M. Sutko (eds.), Digital Cityscapes: Merging Digital and Urban Playspaces, New York, NY: Peter Lang, pp. 100-126.

Ling, R. and S. W. Campbell (eds.) (2009) The Reconstruction of Space and Time: Mobile Communication Practices, New Brunswick, NJ: Transaction Publishers.

London Councils (2013) London Key Facts: Demographics, available at: www.londoncouncils.gov.uk/londonfacts/default.htm?category $=2$.

Lukerman, F. (1964) Geography as a formal intellectual discipline and the way in which it contributes to human knowledge, Canadian Geographer, 8(4): 167-172.

Lynch, K. (1960) The Image of the City, Cambridge, MA: MIT Press.

Marcus, C. C. (1992) Environmental memories, in I. Altman and S. M. Low (eds.), Place Attachment, New York, NY: Plenum Press, pp. 87-112.

Meethan, K. (2006) Narratives of place and self, in K. Meethan, A. Anderson, and S. Miles (eds.), Tourism, Consumption, Representation: Narratives of Place and Self, Oxford: CABI, pp. 1-23.

Meyrowitz, J. (1985) No Sense of Place: The Impact of Electronic Media on Social Behavior, Oxford: Oxford University Press.

Meyrowitz, J. (2005) The rise of glocality: New senses of place and identity in the global village, in K. Nyíri (ed.), A Sense of Place: The Global and the Local in Mobile Communication, Vienna: Passagen Verlag, pp. 21-30.

Ozkul, D. and Gauntlett, D. (2014) Locative media in the city: drawing maps and telling stories, in J. Farman (ed.), The Mobile Story: Narrative Practices With Locative Technologies, New York, NY: Routledge, pp. 113-127.

Relph, E. (1976) Place and Placelessness, London: Pion.

Riley, R. (1979) Reflections on the landscape of memory, Landscape, 23(2): 11-18.

Scannell, L. and R. Gifford (2010) Defining place attachment: A tripartite organizing framework, Journal of Environmental Psychology, 30(1): 1-10. 
Sheller, M. and J. Urry (eds.) (2006) Mobile Technologies of the City, London: Routledge.

Simonsen, K. (2008) Place as encounters: practice, conjunction and co-existence, in J. O. Bærenholdt and B. Granås (eds.), Mobility and Place: Enacting Northern European Peripheries, Farnham: Ashgate, pp. 13-25.

Sutko, D. M. and A. de Souza e Silva (2011) Location-aware mobile media and urban sociability, New Media \& Society, 13(5): 807-823.

Wilken, R. and G. Goggin (eds.) (2012) Mobile Technology and Place, New York, NY: Routledge. 


\title{
Chapter 7
}

\section{Performing city transit}

\author{
Taien Ng-Chan
}

In modern Athens, the vehicles of mass transportation are called metaphorai. To go to work or come home, one takes a "metaphor"-a bus or train. Stories could also take this noble name: Every day, they traverse and organize places...

Michel de Certeau

As de Certeau notes in the epigraph to this chapter, buses and trains can be excellent methods for making meaning out of the repetitions of our daily lives, for every Monday to Friday, in good or bad weather, the urban commuter takes city transit to work or to school. There are few other places in the city, where, if we must commute, we are forced into such close proximity to strangers for extended periods of time, where we are captive audience to advertising and to city streets going by outside the bus windows. We are subject to the smell of bodies, food, the mustiness of wet clothes on rainy days; we are forced to endure the rudeness of other people's cell-phone conversations or too-loud music emanating through headphones. We can create our own bubbles to isolate the self from contact, through mobile media such as cell phones, MP3 players, and other handheld devices (Bissell 2009; Adey 2010; Bull 2000), or through print media such as the daily commuter papers (Straw 2007). Or, we can see the bus as a temporary zone of theater, where a community comes together for the length of time that one travels (Schechner 2003; Jensen 2010). The commute can be seen as simply transportation, a necessary dead time where nothing happens, or it can be a hectic rush to be endured and avoided if possible. It can also be "gift time" (Jain and Lyons 2008), a space for contemplation or enjoyment. It is the idea of "gift time" that I am interested in particularly, for as Ole B. Jensen (2009: 154) points out, pleasure is a less discussed element of mobility, but one that can provide a more meaningful approach to commuting as a life practice. In suggesting a move from "urban transport to urban travel," he asks, "can infrastructures be understood and comprehended within the realm of aesthetic pleasure?"

The question of aesthetic pleasure within urban travel forms the basis of this essay. It revolves around the experience of commuting as "gift time" and also 
“equipped time" (Jain and Lyons 2008), referring to the growing presence of mobile technologies that allow a commuter to control how time is spent while in transit. As cell phones, tablets, and other electronic devices become ubiquitous, they offer a great potential to bring out the pleasures of commuting, for they are uniquely able to interface with the city that then becomes a "hybrid space" (Gordon and de Souza e Silva 2011). This chapter looks specifically at the inbetween spaces of city transit as a liminal space of everyday ritual, a performance site of urban contact, and a hybrid mobile space through the use of locative media, in order to suggest some possibilities toward an aesthetics of mobility.

I begin with a brief methodologies section that situates my approach and frames my case study of city transit in Montréal. The following three sections look at how the spaces of the in-between are constructed, first as a liminal performative space where one transitions between personal and public roles, second as a space of performance and a site of possible engagement with fellow commuters, and, finally, as a hybrid space that can accumulate meaning toward a deeper sense of community and connection. In each section, I establish different elements of the commuting experience, before exploring how works of art and music interact with this experience. I focus specifically on the different kinds of artworks that have taken place within the Montréal transit network, with special attention to locative and mobile media art. In the concluding section, I sum up and discuss the impact of these site-specific urban transit projects, and argue that works of locative art are uniquely able to engage the commuter in a hybrid landscape in order to connect with, rather than disconnect from, the surrounding environment. I argue that mobile locative media art can be a major instigator of a move toward pleasure in urban travel.

\section{Methodologies}

As the main setting for my explorations, I draw upon Montréal's public transit system - the Société de transport de Montréal (or STM). City transit is a central part of Montréal life. In 2010, the STM won the American Public Transportation Association Award as Outstanding Public Transportation System in North America (STM 2010b). According to the STM, 404.8 million trips were made in 2011; Montréalers took public transport 214 times that year, on average, compared to 188 times by Torontonians and to ninety-three times by residents of major US cities including Boston, Chicago, New York, Washington, Philadelphia and Seattle (STM 2012: 14). A full two-thirds of people who work or study in downtown Montréal commute via public transportation (Heffez 2010). The STM is perhaps exemplary for its integration of art into its network. For instance, a bus ticket or OPUS monthly pass will earn discounts off many of the city's cultural events such as film festivals and museum days. The STM partners regularly with festivals such as the all-night arts event Nuit Blanche, when bus and metro services also remain open all night, and a number of artists have made works directly engaging the transit system as set and setting. The STM thus 
provides many opportunities to study the aesthetics of mobility and its interrelations with mobile and locative technologies.

As a frequent user of the Montréal city transit system, I count myself among those who "walk with" my subjects at the same time that I am reflexively also my subject. I am an auto-ethnographer. At the same time, I look at my environment as an artist, with an eye on aesthetics, light, shadow, color. Through a kind of "sociological stalking," I observe my fellow commuters and my surroundings, sometimes using video and audio to capture the atmosphere. I often record my thoughts with the use of time-space diaries (Büscher et al. 2010). These are all raw materials for my work. In my discussion of the art that I have encountered and draw upon here, I use these mobile methods to help describe my experiences and relate them to my explorations of urban transit as a site of engagement with the city and our fellow citizens.

I frame my theoretical discussions mainly through Victor Turner's anthropological studies of the liminal space $(1977,1982)$, as well as Erving Goffman (1973 [1959]) and Georg Simmel (1998 [1907]), both sociologists who focused on the minute interactions of everyday life in urban settings. In addition, I draw upon Turner's concept of communitas, which can also be found on the small, everyday level. Communitas offers contact and exchange with our fellow citizens, moments of more unguarded being, and it is often through art and music that it occurs. Liminality and communitas are core concepts in an aesthetics of mobility.

While there has been much investigation into "the art of travel" through the social sciences (Watts 2008; Bissell 2009; Jensen 2009; Watts and Lyons 2010; Edensor 2011), not much has been written about the actual art that engages with the potency of liminal space and the potential of communitas in urban commuting. Thus, through a discussion of the various artworks that I have encountered in the Montréal transit system, and building on some recent theories on mobile media interfaces that create a new sense of space and place (Gordon and de Souza e Silva 2011; Farman 2012; de Souza e Silva and Frith 2012), I sketch out a few possible pleasures toward an aesthetics of mobility.

\section{City transit as a liminal space}

When I arrive at the bus-stop, there is already a long line-up. It's dark and raining. The bus doesn't come for over ten minutes, but when it finally does, it is packed full. I squish my way in, validate my fare card and push my way through the crowd, which tends to accumulate the most densely in the first third of the bus. Some people are completely unaware of the space around them, obliviously wearing their bulky backpacks so that it is difficult to get by them. There are advertisements and posters on the bus, advising people to "tenir son sacs à dos" (hold backpacks by the hand); clearly, many people don't realize they are the targets of such advice. There is, of course, nowhere to sit. I get out my iPod, put on my headphones, and turn up the music loud, thinking about the tasks I need to get done today at work. 
The retreat into isolation on public transit has variously been called a "safety bubble" (Bissell 2009), "bubble of privacy" (Adey 2010), or "cocoon" (Farman 2012; Jain and Lyons 2008). Michel de Certeau (1984: 111), in the chapter "Railway navigation and incarceration," calls it a "bubble of panoptic and classifying power, a module of imprisonment." He goes on to describe the train window as that which "makes our memories speak or draws out of the shadows the dreams of our secrets" (1984: 112). Here, de Certeau refers to how travel time can also become an individual spatial practice of accumulating memory and meaning. The liminality of city transit can be a valuable tool in the practice and rituals of everyday life.

To begin my discussion about the liminal space of city transit as a ritual, I draw upon the work of Turner (1977), a cultural anthropologist and ethnologist, who was originally describing the cultural traditions of Zambian indigenous tribes, but later widened his scope to settings in the Western industrialized world. I look as well to Goffman (1973 [1959]), whose well-known work framed the small interactions of everyday life as worthy of investigation. It is in Goffman's spirit particularly that I relate Turner's work on the liminal space to the quotidian act of commuting.

Turner, as described in his book From Ritual to Theatre (1982: 24-25), became interested in the study of symbolic genres through Arnold Van Gennep's Rites de Passage. Van Gennep studied rites of passage in small-scale societies, and the term has come to signify those landmark events such as birth, marriage, and death, but Turner is adamant that its application is much broader, stating that "I have tried to revert to van Gennep's earlier usage in regarding almost all types of rites as having the processual form of "passage." "Now, a commute on city transit can certainly be seen as routine, but can it be called a rite?

Rich Ling (2008), in his work on mobile communications and ritual interactions, discusses the many different uses of the word "ritual," which he notes, can be seen as pejorative (as in merely symbolic, with no other active value) but is for him positive, a "catalyst for cohesion." He notes that his usage is that of a social phenomenon, a societal construction for interaction, following sociologist Emile Durkheim, who, like Turner, also references van Gennep. He also notes that Durkheimian "ritual" can be similarly applied to everyday interactions, after Goffman. If we broaden the definition of a "rite of passage," as Turner, Goffman, and Ling do, then we can include the commute as such a daily rite. Here, however, I focus particularly on Turner's meaning of ritual as transformative, from one state or social status to another.

In "The phenomenon of mobility at the Frankfurt International Airport," Kerstin Söderblom (2008) invokes Turner for her analysis of transit passages, specifically air travel, on the three steps common to all passage rituals: separation, passage/transition, and reconnection/reincorporation. Air travel is a more extreme form of liminality than the Goffmanesque form of the daily commute, as it is not for most of us routine, and its effects are more pronounced. Nevertheless, the structures of liminality are similar and thus offer a valuable comparison. 
Following Söderblom's transposition, we can thus describe a ride on Montréal city transit as follows: The first phase of separation clearly demarcates sacred from profane, using symbolic behavior to detach the individual from his or her previous social status. Söderblom describes the symbolic behavior of separation as the process of passing through security, having all your belongings and each part of your person scanned or touched, in order to grant you entry. On public transit, this symbolic behavior resides more simply in validating your bus pass or ticket, which you must keep with you at all times or risk prosecution, and then passing through the turnstiles of the metro or past the bus driver and onto the bus.

Because the Montréal metro is completely underground, one must descend quite far into a different landscape below where there are different rules and codes; however, even the bus, with its metal-box construction and window frames looking out onto the passing scenery, can be seen as having a unique and particular ambiance that frames liminality. Phase two, passage or the liminal stage, is a free-floating space, the result of a suspension of certain rules, where status, roles, and private identities do not count for much. Söderblom (2008: 185 ) notes that this is only possible because "the space is framed, controlled, and clearly distinguished from other places."

This liminal state is a space where one is not obliged to play a social role. Goffman, in his book The Presentation of Self in Everyday Life (1973 [1959]), elucidates his theory that each of us must act differently according to different settings, and that we present ourselves in a series of different masks depending on the context. He suggests that all social interactions are staged, and that one's main roles and interactions are thus prepared for "backstage" and played out on a "main stage" area. The rite of passage, then, prepares you for the performance, and allows you to emerge with your mask on, or to take it off, or to change roles. In this sense, both Goffman and Turner, in using the theatrical paradigm, point to the importance of ritual that enables these small transformations of the self.

Tellingly, various commentators in different studies (Jain and Lyons 2008; Watts and Lyons 2010) said that even if they could teleport to their destination, they would not want to. They preferred to have the travel time. The liminal space offers an opportunity to plan, to think, to daydream, to be between roles and responsibilities. "Transition time" was found to be one of two categories of travel time, as articulated by Jain and Lyons (2008) in their study of travel time as "a gift" to the individual commuter. "Time out" was the other category, which described how some people might even take a longer route over a shorter one in order to have longer to relax, listen to music, think, or consider a work issue. Travel time is clearly the framework for any aesthetics of mobility.

To manage and control their experiences of travel time, commuters equip themselves to deal with their surroundings and themselves in the middle of the liminal space, in order to maintain their ritual of transition or time out. This is “equipped time" (Jain and Lyons 2008), which more and more includes mobile and locative technologies. Urban commuters and travelers have always used "equipped time," most often to disengage from the urban environment. Indeed, 
Simmel's oft-quoted work The Metropolis and Mental Life was originally written in 1903 but is still pertinent today. Simmel's discussion of the metropolitan "blasé" attitude forms the basis of many contemporary investigations into urban space and interaction (see, for instance, Gordon and de Souza e Silva 2011; de Souza e Silva and Frith 2012). Simmel's observations work alongside Goffman's to describe the techniques of non-engagement and selective interaction that people use in spaces of urban transit.

For instance, Simmel describes how the different senses affect our interactions in public space, that is, how we act to avoid interacting. He points out that looking is perhaps the most direct, pervasive interaction there is. Because usually, strangers do not look at each other directly, catching someone's eye on the bus can amount to a brief connection, the sharing of a particular moment. To not look at someone is to remove oneself, in a sense, from being seen; it is to retreat into the isolating bubble, often by looking at other things, objects, and devices. Jensen (2010) notes that the "classic" tools (such as newspapers and books) that Goffman describes as important for establishing and maintaining isolation are now supplemented with mobile technologies such as MP3 players, smartphones, and tablets.

With "equipped time," we have some control over the shape of our liminal space. We can use the travel time to transition or to time out. As Laura Watts (2008) suggests, we each shape our travel times differently, according to the different artifacts, practices, and methods that we use. Watts shapes "ethnography time" with her work, observations, pen and paper; I shape "creative time" when I am filming images or recording sound, in the flow with no awareness of time passing. If I am not equipped or if I feel like doing nothing, travel time may appear slow. If I am listening to music, the time is shaped by the kind of music, whether symphonic, mellow, melodic, or discordant.

Music is a common tool for commuters on city transit. Michael Bull (2000, 2007) has written extensively about how often people use music to give themselves control over their journey. He finds that many people will use the same music on a regular basis, so that each part of the journey has its own tune. He also notes that music can let one create imaginary cinematic narratives, and allows what he calls "nonreciprocal looking," where you can look at other people semi-directly, but your visible earplugs announce that you are really otherwise occupied. These kinds of actions are aimed at isolating the self from contact, and maintaining the space of liminality.

Mobile technologies have changed this liminal space from Simmel's time. Earplugs are one instance of how such props have become incorporated into social interactions. "Equipped time" has also been reshaped by mobile media such as the smartphone and the tablet, so that the space of city transit now gives a vast array of possible distractions and pleasures. The various media devices have given the commuter more control than ever in controlling the experience of transit time, whether as transition time or time out. The isolating bubble of city transit is stronger than ever. 
And yet, paradoxically, what makes city transit unique as a liminal space is the intensity of the urban experience, the sensual and material qualities of the space, and the proximity of fellow commuters, all of whom are also actors in the same space, each in their own bubbles. The performativity of city transit functions both to transform the individual self, but also to generate a space of performance. It is this space that provides the possibility of communitas in a way that few other urban sites can, and for this reason, needs to be considered along with liminality as a foundational concept in the aesthetics of mobility. In the next section, I explore how Turner's concept of communitas can set the stage for locative mobile technologies to engender a deeper sense of place, community, and pleasure.

\section{City transit as a space of performance and communitas}

The space of city transit can be thought of as a performance space in many ways. Not only is it performative in a liminal sense (of transforming the role-playing self), but also as a space of social interaction. Because we must spend a certain amount of time together physically in the material space of a bus or metro car, anything that happens here can be seen as a social drama co-performed by actorcommuters. To extend Goffman's dramaturgical metaphor, Jensen (2010) brings in the idea of the subway as a stage with a play already ongoing, and the other commuters as performers who are already there in mid-scene when you come on stage.

Erika Fischer-Lichte (2008) notes that the materiality of the performative space emerges through different aspects and elements, which she describes at length to include such terms as presence, embodiment, and corporeality. This could extend to commuters and bus drivers as actor-bodies and the bus as an architectural theater space (the shape and layout of the vehicle itself, the STM "branding" and the various types of media and advertising that "decorate" the walls), which emits a spatiality that changes according to the time of day (rush hour, off-hours) and the neighborhoods that the bus is traveling through. In addition, there is the element of rhythm in the repetitions of the bus or metro car stopping and starting, and the entering and exiting of passengers at each stop. Each of these elements contributes to the atmosphere of the space and influences performance.

Tim Edensor (2011: 189) also picks up on rhythm, particularly through Henri Lefebvre's Rhythmanalysis, to see how it can be used as "a starting point for investigating the complex temporal rhythms of the multiple mobilities coursing through space." He foregrounds itineraries produced by time, collective rhythms of the city as enacted through early morning and later afternoon rush hours, the mid-afternoon lull. City transit can be meditative and peaceful, especially in the late afternoon. During rush hours, however, the experience is more likely to be seen as irritating than as a gift. 
Rush hour will directly affect the kind of experience you are likely to have: Whether you will feel stressed and pressured, whether you are likely to sit, stand, or be packed in sardine-like. During rush hour, you are required to perform agile feats of interweaving between bodies, as you push through a crowded bus, or as you rush from one metro platform to the next to transfer trains, or toward the exit. These "body techniques," as Marc Augé calls them, "are closely connected to this presence of others ... your ways of standing, sitting, and moving are directed by the density of the crowd" (Criqui 1994).

Body techniques, simple gestures, and non-interactions (where we act to avoid engagement), are the most common types of performances on city transit. However, although the overwhelming state of mind found during the liminal phase is that of anonymity and non-interaction, all of the traumas of urban life can also be found there in a compressed fashion. There are times of random connections and kindnesses, incidents of sexism, racism, and all manners of conflict and resolution.

Richard Schechner, in his book Performance Theory (2003), draws on both Goffman and Turner to take an interdisciplinary approach to viewing theater, including anthropological rites, political demonstrations, sports, theatrical productions, and performing arts events. Schechner (2003: 152) attempts to visualize the connections between a wide spectrum of social interactions and events, and notes that "the differences among ritual, theater, and ordinary life depend on the degree spectators and performers attend to efficacy, pleasure, or routine." As commuters and urban travelers, if we are focused on efficacy, then we are simply waiting for arrival. Random encounters with strangers can turn the focus from efficacy and boredom to theater and pleasure, and possibly even communitas.

Turner (1982: 45) defines communitas as "the liberation of human capacities of cognition, affect, volition, creativity, etc., from the normative constraints incumbent upon occupying a sequence of social statuses, enacting a series of social roles." It has to occur during the liminal phase, when people are no longer role-playing, no longer aware of social status. It manifests as a kind of interrelatedness that can reach across the structural systems that usually dictate social behaviors. People experiencing communitas feel a sort of unity, though not in the sense of "merging," for individual distinctiveness is preserved. Communitas may be rare, but as Turner (1982: 46-47) notes, it is "sometimes aided by the projections of art that this way of experiencing one's fellows can be portrayed, grasped, and sometimes realized." When communitas occurs spontaneously, it is "a direct, immediate and total confrontation of human identities ... it has something 'magical' about it."

How does the performance space of city transit bring about communitas through art? Most often, it is through performance itself, sometimes literally. For instance, the STM often partners with various cultural institutions such as Les Grands Ballets Canadiens or Opéra de Montréal to bring spectacles and concerts to the metro stations (STM 2010a). These kinds of performances can draw people together into a space where there is an atmosphere of communitas. 
Buskers can sometimes do this as well. As Amanda Boetzkes (2010) demonstrates in her essay about "the ephemeral stage" at Lionel-Groulx Metro, buskers must work to break the isolation between strangers, and to solidify the bonds between them. An engaging performance allows people to break out of their bubbles of isolation, to unify in a collective form. Through subtle gestures and body language, "individuals in the crowd collaborate and come to a precarious agreement to collectively gravitate around the performance while respectively maintaining their personal definition" (Boetzkes 2010: 153). This description of the audience fits Turner's definition of communitas, and although it does not happen often, when it does, it becomes an event, something memorable that turns non-place into place for the individual who experienced it.

Buskers and other kinds of performers attempt to break the bubble of isolation in order to entertain. Mobile media has changed the kinds of performances that are possible in the public sphere, to redefine who can be a performer. As with the advent of such practices as social networking and crowdsourcing, public performance now includes Flash Mobs and Smart Mobs, where groups of people assemble quickly and suddenly to perform a specified action for a usually brief period of time, and then just as quickly disassemble. They are usually coordinated through mobile media devices, most often cell phones and SMS texts (Lemos 2010). Flash Mobs can occur anywhere, at any time, but one of the earliest and most well-known instances is the No Pants Subway Ride, a now annual worldwide event that anyone can participate in. Montréal joined in with its first Flashmob d'individus sans pantalon on the Metro in January 2013. ${ }^{1}$ These types of performances, with their elements of surprise and silliness, can also break through the bubble of isolation.

Beyond performance, public art installations can also foster a type of communitas. For example, a rare instance of public art as a kind of theatrical setting was found in a metro car installation called Point de fuite (2007) by visual artist Rose-Marie E. Goulet and sound artist Chantal Dumas. Point de fuite (or "vanishing point") was the first project of its kind to be realized in a metro in North America (Point de fuite 2007). As an installation, it intervened in the normal spatiality and materiality of the metro system with a variety of sounds and images, such as snippets of narratives, chirping birds, applause, roosters, laughter, chimes, and pixelated photos of the city on the windows, walls, and ceiling of a metro car. Part of the fun was to watch the reactions of others, as the work often provoked commentary and conversation. The shared experience of the art installation often brought the individual passengers together into something more of a community.

Communitas remains, however, a relatively rare occurrence. Most of the time, people prefer not to interact directly with others, out of fear or anxiety (Bissell 2009). Indeed, the defining experience of commuting on city transit is one of isolation and non-interaction. But communitas is possible in the isolating bubble, too, or something like it, and it is locative mobile media, specifically, that enables this possibility. In the next section, I investigate some art works that 
engage with liminality, communitas, travel time, and the experience of emplacement through mobile and locative media, each being an exploration of how place can be enacted on Montréal city transit.

\section{City transit and locative media as hybrid space}

Mobile technologies may help to strengthen the bubble of isolation, but as de Souza e Silva and Frith (2012) point out, cell phones and props such as headphones do not automatically disconnect people from their surroundings. If the goal is to completely insulate the individual from the outside world, it will never be fully reached for sounds of the city inevitably filter in. Instead of presuming that mobile media is used simply to shut out and to isolate, another approach is to ask how this technology might be used to draw one into an awareness of place and everyday sound (Kaye 2012).

Samuel Thulin (2013) examines this question in two ways. He investigates new smartphone applications that connect audio content with location, drawing connections to mobile sound art that focuses attention on the moment of interaction between sound and place. He also offers his own creative intervention into the travel time bubble with his project "There to Hear: Placing Mobile Music." This forty-two minute musical composition uses only field recordings from a specific city transit route in Montréal and is meant to be listened to in the same places where the sound was found (Thulin 2010). Through what he calls "slippages" (confusion as to whether a particular sound came from the music or the environment), Thulin challenges the separation between place and listener, while asserting that the isolating bubble is actually quite porous (Kaye 2012).

Another mobile media piece called "Stéréobus" by Montréal sound collective Audiotopie also directs you on a sound tour, this time a narrative taking place on a specified bus route in Laval, a suburb of Montréal. Through the headphones, you hear two voices that guide you on a journey, which lasts an hour and a half. A male voice asks you questions (I have translated these from the original French): "Listen to what is going on here. What emerges? Here, now? How is perception organized? Identify an order, recognize a rhythm ... What is happening here? What is behind you?" At the same time, electronic music and urban background sounds provide layers and echoes that draw attention to your surroundings. Another voice, a woman, intervenes and provokes a drama that begins to unfold, seemingly about a man, once an aviator, who has been trapped in his psycho-sensorial experiences in the year 2574 ("Cartier: Stéréobus," n.d.).

Both these projects are site-specific, and play with the "time out" of travel time to create new awarenesses of place, but in quite different ways. Thulin's piece is a musical composition that engages place through found sound, and although the movements of the music correspond roughly with the movements along the route, Thulin does not aim for a "perfect fit." Instead, he acknowledges that along the way, chance occurrences such as a bus arriving earlier or later than scheduled result in a variety of ways in which his music and the environment 
can combine, resulting in a "meta-composition" that emerges differently each time the piece is heard contextually (Thulin 2010).

On the other hand, Audiotopie's work offers a succession of audio illusions to tell a story, similar to the work of Janet Cardiff and George Bures Miller. Cardiff and Miller were perhaps the first to become known for their site-specific audio walks, but as Gordon and de Souza e Silva (2011) note, their initial projects did not use any location-aware technology. In both Cardiff/Miller's and Audiotopie's projects, instructions reside within the soundtrack that direct the listener to, for instance, "move through the turnstiles of the metro." Because both projects rely on the listener's ability to follow these instructions, automated GPS positioning would certainly make the routes easier to navigate, or to provide correction if a participant gets lost.

Locative mobile media art opens up possibilities for different approaches to the sound walk, one that does not need to rely on a prescribed route or linear narrative, and thus offers more flexibility. With "mobile annotations" (Lemos 2010; Gordon and de Souza e Silva 2011) or "urban mark-ups" (Farman 2012), the practice of attaching information to location presents potential for new hybrid landscapes and ways of experiencing the city. For example, my own artistic practice focuses on personal engagements with the city, through stories, poems, short videos, and soundclips that are site-specific. My most recent work, Detours: Poetics of the City is a website that maps these engagements and those of my collaborators, and includes videopoems that were made with cell-phone video taken on the bus and metro. ${ }^{2}$

These mobile media artworks demonstrate the individual's ability to inhabit two spaces at once, the digital and the material, to create a new kind of hybrid space (Gordon and de Souza e Silva 2011). This hybridity can allow the traveler to practice "emplacement." Jason Farman (2012) describes emplacement as the "counterpoint to displacement" and is linked to embodied engagement and Heidegger's Dasein or “being-in-the-world." Farman argues that through digital and mobile media poetics, the cell phone and other such mobile interfaces can augment methods of exploring public space, rather than removing oneself from it. They can connect histories, stories, and ephemeral media to individuals and communities, to situate and to emplace.

Project 55 is an example of such a locative media work, produced by the Center for Oral History and Digital Storytelling (COHDS) at Concordia University. ${ }^{3}$ Subtitled A Historical Audio Tour of Ethnic Communities along St-Laurent Boulevard Aboard Bus 55, it is meant to be listened to in situ. The tour comes as a downloadable MP3 file, meant to be started as one boards Bus 55 at the beginning of its route. As the bus progresses up Montréal's St-Laurent Boulevard, it passes through Chinatown, Little Portugal, the old Yiddish-speaking Mile End of author Mordecai Richler's childhood, and Little Italy, and we hear on our mobile device a series of accompanying interviews with citizens from those communities, store-owners and a bus driver who has been driving the 55 route since the 1990s. These oral histories bring to life the neighborhoods that we are 
passing through, giving a glimpse into different times and cultures. The Project 55 tour is an example of how something very like communitas can be produced though locative media, for storytelling is an excellent way to produce empathy and a way of "experiencing one's fellows" (Turner 1982: 46).

Through these projects and artworks as models for mobile locative methods of engagement with the city, we can find pleasure in urban travel and the commute, and what deeper pleasure is there than feeling "emplaced" and a part of the world that is our home? Hybrid landscapes may be the foundational concept of an aesthetics of mobility, one that can guide our daily practices and rituals, like that of commuting, of travel time and performing communitas, to gain a deeper understanding of the places in which we live.

\section{Conclusion}

In his book Non-Places, Marc Augé (1995: 63) looks at the global expansion of spaces "which cannot be defined as relational, or historical, or concerned with identity." He includes airports, as well as:

gas stations, shopping malls, train stations, motels, highways and all the various means of transportation and transit areas as non-places.... It is a status of "in-between," where nothing is fixed and stable and where time and place are fluid and hybrid and seem to follow their own rules

(Söderblom 2008: 182)

However, Tim Edensor (2011) critiques this perspective as overlooking the complex practices and the material atmospheres that make up such non-places. As well, he aims to counter popular representations of commuting as a dystopian and alienating routine. This essay is also about countering such representations, specifically through notions of pleasure and place-making and how they can transform the liminal and performative spaces of city transit.

The idea of pleasure in mobility can be built upon the structures of travel time as a gift, both for transition and time out. Travel time offers the pleasures of the journey, of being in-between roles, time to think, daydream, prepare, read, or listen to music. The liminal space is also a site of performance, which can provide for engagement with fellow travelers through the pleasure of communitas. But perhaps the most defining aspect of city transit occurs within the bubble of isolation. Through the use of mobile and locative media, the bubble can offer the pleasures of engagement with art and storytelling situated within a mobile, yet site-specific space. Through emplacement, we can practice the pleasures of making place. These elements offer the first steps toward an aesthetics of mobility, though there is still much to be explored.

Though they are all similar in their basic methods of organizing space (as non-place), each city transit system has its particulars, its own kind of beauty and frustrations, its own kind of city transit art. Los Angeles, for instance, has its 
"Out the Window" project, featuring short video and animation clips that play on a small screen mounted inside the bus. ${ }^{4}$ Toronto has the annual Toronto Urban Film Festival, which plays a selection of "short, sweet and silent" films on subway platform screens..$^{5}$ These examples provide suggestions for further research into what the aesthetics of mobility might entail.

Jensen (2009) proposes that the city is materialized through mobility, that we make contact with urban space by moving through it. Indeed, it is through mobility that memories and meanings can accumulate. Augé's book In the Metro (2002) looks at Paris from the viewpoint of a commuter who has spent years riding the trains. It is in part a meditation on self and memory, and in part an analysis of the globalization that has produced non-places and massive urban development. Augé reveals the ways in which daily life produces maps and itineraries, accumulating personal meanings that layer themselves onto public names and monuments. Engagements with art, music, and stories are excellent ways to amass meaning. The in-between space of city transit is ripe for creative interventions of all kinds. In this way, we can see the vehicles of urban transit as "metaphors" (after de Certeau), which allow us to write our own stories and to place ourselves into our own lives.

\section{Notes}

1 See: http://www.nightlife.ca/divertissement/flashmob-dindividus-sans-pantalon-dans-lemetro-de-montreal-dimanche

2 See: http://agencetopo.qc.ca/detours/citytransit_en.html.

3 See: http://storytelling.concordia.ca/memoryscapes/WebsiteSections/01Projects/2006/ project55/index.html.

4 See: http://out-the-window.org/.

5 See: http://www.torontourbanfilmfestival.com.

\section{References}

Adey, P. (2010) Mobility, Abingdon/New York, NY: Routledge.

Augé, M. (1995) Non-Places: Introduction to an Anthropology of Supermodernity, London/New York, NY: Verso.

Augé, M. (2002) In the Metro, Minneapolis, MN: University of Minnesota Press.

Bissell, D. (2009) Moving with others: The sociality of the railway journey, in P. Vannini (ed.), The Cultures of Alternative Mobilities: The Routes Less Travelled, Farnham/Burlington, VT: Ashgate, pp. 55-70.

Boetzkes, A. (2010) The ephemeral stage at Lionel Groulx Station, in A. Boutros and W. Straw (eds.), Circulation and the City: Essays on Urban Culture, Montréal: McGillQueen's University Press.

Bull, M. (2000) Sounding Out the City: Personal Stereos and the Management of Everyday Life, New York, NY: Berg Publishers.

Bull, M. (2007) Sound Moves: iPod Culture and Urban Experience, Abingdon: Routledge.

Büscher, M., J. Urry, and K. Witchger (eds.) (2010) Mobile Methods, Abingdon/New York, NY: Routledge. 
Cartier: Stéréobus. (n.d.) available at: http://www.audiotopie.com/stereobus.

Criqui, J. P. (1994) Home made strange - interview with ethnologist Marc Augé, ArtForum, available at: http://www.thefreelibrary.com/Home+made+strange.-a016097500.

de Certeau, M. (1984) The Practice of Everyday Life, Berkeley, CA: University of California Press.

de Souza e Silva, A. and J. Frith (2012) Mobile Interfaces in Public Spaces: Locational Privacy, Control, and Urban Sociability, New York, NY/Abingdon: Routledge.

Edensor, T. (2011) Commuter: Mobility, rhythm, commuting, in T. Cresswell and P. Merriman (eds.), Geographies of Mobilities: Practices, Spaces, Subjects, Farnham/ Burlington, VT: Ashgate, pp. 189-204.

Farman, J. (2012) Mobile Interface Theory, New York, NY/Abingdon: Routledge.

Fischer-Lichte, E. (2008) The Transformative Power of Performance, New York, NY: Routledge.

Goffman, E. (1973 [1959]) The Presentation of Self in Everyday Life, Woodstock, NY: Overlook Press.

Gordon, E. and A. de Souza e Silva (2011) Net Locality: Why Location Matters in a Networked World, Chichester: Wiley-Blackwell.

Heffez, A. (2010) Montréal metropolitan area: More sprawl, more transit, February 18, available at: http://spacingmontreal.ca/2010/02/18/montreal-metropolitan-area-moresprawl-more-transit/.

Jain, J. and G. Lyons (2008) The gift of travel time, Journal of Transport Geography, 16(2): 81-89.

Jensen, O. B. (2009) Flows of meaning, cultures of movements: Urban mobility as meaningful everyday life practice, Mobilities, 4(1): 139-158.

Jensen, O. B. (2010) Erving Goffman and everyday life mobility, in M. H. Jacobsen (ed.), The Contemporary Goffman, New York, NY/Abingdon: Routledge.

Kaye, L. (2012) YOU ARE HERE: Binaural audio, mobile media and the sonic exploration of urban space, Wi: Journal of Mobile Culture, 7(1), available at: http://wi.mobilities.ca/ you-are-here-binaural-audio-mobile-media-and-the-sonic-exploration-of-urban-space.

Lemos, A. (2010) Post-mass media functions, locative media, and informational territories: New ways of thinking about territory, place, and mobility in contemporary society, Space and Culture, 13(4): 403-420.

Ling, R. (2008) New Tech, New Ties: How Mobile Communication is Reshaping Social Cohesion, Cambridge, MA: MIT Press.

Point de fuite: Rose-Marie E. Goulet and Chantal Dumas (2007) October 11, available at: $\mathrm{http}: / /$ dpi.studioxx.org/demo/?q=en/no/09/chronique-actualite-point-de-fuite-chantaldumas-et-rose-marie-goulet.

Schechner, R. (2003) Performance Theory, New York, NY: Routledge.

Simmel, G. (1998 [1907]) Sociology of the senses, in D. Frisby and M. Featherstone (eds.), Simmel on Culture: Selected Writings, London/Thousand Oaks, CA: Sage Publications.

Söderblom, K. (2008) The phenomenon of mobility at the Frankfurt international airport: Challenges from a theological perspective, in S. Bergmann and T. Sager (eds.), The Ethics of Mobilities: Rethinking Place, Exclusion, Freedom and Environment, Aldershot/Burlington, VT: Ashgate.

STM (Société de transport de Montréal) (2010a) Artistic, cultural and urban transit by STM, April, available at: http://www.docstoc.com/docs/90656461/artistic_-culturaland-urban-transit-by-stm. 
STM (Société de transport de Montréal) (2010b) STM wins American public transportation association award as outstanding public transportation system in North America, October, available at: http://www.stm.info/en/press/press-releases/2010/stm-wins-american-publictransportation-association-award--as-outstanding-public-transportation-system-in-northamerica.

STM (Société de transport de Montréal) (2012) Activity Report 2011, available at: http:// www.stm.info/sites/default/files/a-ra2011.pdf.

Straw, W. (2007) Global Metro: The rise of the free commuter newspaper, Revista Mexicana de Estudios Canadienses (nueva época), 13: 45-53.

Thulin, S. (2010) There to hear: Placing mobile music, available at: http://archive.oboro. net/archive/exhib1011/samuel_thulin/info_en.html.

Thulin, S. (2013) Mobile audio apps, place and life beyond immersive interactivity, Wi: Journal of Mobile Culture, 7(1), available at: http://wi.mobilities.ca/mobile-audioapps-place-and-life-beyond-immersive-interactivity/.

Turner, V. (1982) From Ritual to Theatre: The Human Seriousness of Play, New York, NY: PAJ Publications.

Turner, V. (1977) Variations on a theme of liminality, in S. F. Moore and B. G. Myerhoff (eds.), Secular Ritual, Amsterdam: Van Gorcum, pp. 48-65.

Watts, L. (2008) The art and craft of train travel, Social and Cultural Geography, 9(6): 711-726.

Watts, L. and G. Lyons (2010) Travel remedy kit: Interventions into train lines and passenger times, in M. Büscher, J. Urry, and K. Witchger (eds.), Mobile Methods, Abingdon/New York, NY: Routledge, pp. 104-118. 


\title{
Location-based gaming apps and the commercialization of locative media
}

\author{
Dale Leorke
}

Location-based games were among the first interventions into urban space that employed locative media for playful behavior aimed at challenging the conventions of public space. They use mobile phones, GPS (Global Positioning System), Bluetooth and WiFi technology and other location-aware technologies and devices to incorporate the physical location of players into the game. Early, pioneering iterations of these games such as The Beast (Microsoft 2001), BotFighters (It's Alive! 2001-2005) and PacManhattan (Frank Lantz 2004) experimented with the ubiquity of mobile and locative media to create games that blended digital gameplay with the physical environment, geography, and everyday locales of urban space. While location-based gaming projects continue to be developed by artists and small communities of players, today they increasingly appear as commercial apps for the iPhone or Android platforms. This shift has transformed location-based games from a niche genre, little known outside artistic circles, and brought them into the mainstream mobile gaming market. The advanced GPS technology and superior graphics of smartphones, combined with digital distribution services like Apple's App Store, provide location-based games with a vastly greater audience of potential players. But this transformation also illustrates how location-based games - once the purview of artists, amateurs, and small commercial outlets - have been adapted into the relatively new value chain of the global app economy. It thus raises broader concerns over the extent to which location-based games have been co-opted by commercial interests and the growing conflation of play and leisure with labor, at the expense of their capacity to challenge the norms and conventions of public life in the city.

In this chapter I examine the theory around location-based games in the context of the emerging "app economy" and the growing commercialization of locative media taking place with the success of the iPhone and Android platforms. I argue that the proliferation of these devices signals a shift in locationbased gaming, from its avant-garde origins in the locative art movement of the 2000 s to today's growing consumer-driven appropriation of locative media technology. I begin by tracing the origins of location-based gaming within the locative art movement, which situates early location-based gaming projects within a 
historical trajectory of avant-garde practices aimed at repurposing the public spaces of the city for play. With the shift toward apps, however, I investigate how location-based games are becoming increasingly commercialized as they seek to compete within the highly competitive, concentrated, and profit-driven mobile games industry. I examine one particular location-based game development studio, Red Robot Labs, and their game Life is Magic, released for iOS and Android in early 2013. I argue that the entrance of location-based games into the app economy represents a shift away from their roots in the locative art movement, one which illustrates the growing capture of digital play by the increasingly competitive mobile gaming market.

\section{The origins of location-based gaming}

Location-based games emerged in the early 2000s alongside the "locative art" movement, which exploited the growing ubiquity of GPS tracking and locative media devices in order to bring interactive, "new media" art into public space (see de Souza e Silva and Frith 2012; Drakopoulou 2010; Farman 2012; Flanagan 2009; Gordon and de Souza e Silva 2011). Locative art developed at a time when many theorists in urban studies and sociology had, over the previous thirty or so years, been lamenting the "decline" of public life (see Augé 1995; Sennett 2002 [1977]; Sorkin 1992; Virilio 1997 [1984]). While these theorists differed in their views and approaches, their writing shared a common thread. They argued that throughout the twentieth century, the rise of electronic communication technologies had revolutionized domestic life to the detriment of social and public life in the contemporary city. As Varnelis and Friedberg write, "public space became increasingly privatized and virtualized" during the twentieth century, "with networks of individuals being replaced by television broadcast networks, and individuals becoming less and less citizens and more and more consumers" (2008: 18). With the rapid growth in the number of mobile phones in the 1990s, however, mobile technologies were seen as potentially responding to this erosion or "decline" of public life (see de Souza e Silva and Frith 2012; Varnelis and Friedberg 2008; Tuters and Varnelis 2006). Essentially, they brought media technologies and the practices that occurred around them-which had mostly been confined to the home or specific, fixed locations - out into the streets and public spaces of the city. At the same time, however, these technologies were also criticized for disconnecting or distracting their users from the physical environment around them in favor of the private world of their mobile phone screens or portable devices (see Ito 2005; Kluitenberg 2006; Rheingold 2002).

The ability to communicate and share information virtually anywhere, coupled with the prevalence and decreased cost of technologies like GPS, WiFi, smartphones, digital video recording, and handheld gaming, led to new patterns of media consumption and production. But more specifically, they also laid the groundwork for a radical art movement that employed these technologies to mobilize their users to participate in artistic performances and interventions 
within urban space. The aim of these projects was to move interactive, digital art from the confines of the gallery and computer screen and into the streets, public spaces, and physical architecture of the city. This movement became known as locative art, following the widespread adoption of the term "locative media" during the Art+Communication Festival in Latvia in 2003 as a descriptor for devices and technologies embedded at or in a particular location (Zeffiro 2012: 251). With the proliferation of mobile media and location-aware devices in the late 1990s and early 2000s, many artists saw locative media as having the potential to reinvigorate interactive art by using it to critique the established conventions and ideologies of public space (see Hemment 2004, 2006; Tuters and Varnelis 2006).

Locative artists sought to repurpose GPS technology from its military and consumer origins as a way of re-engaging its users with public space. They built on a history of public art installations and projects, such as Teri Rueb's "environmental sound installation" Trace (1999), Janet Cardiff's experimental, sitespecific museum “tour guide" In Real Time (1999) and Rafael Lozano-Hemmer's large-scale urban installation Vectorial Elevation (1999). These earlier, mostly outdoor interactive projects used custom-made GPS devices, audio headsets, and the internet respectively to experiment with bodies, movement, and technology in physical space. Following on from these experimental installations and exhibitions, locative art projects like dot.walk (Wilfried HuJeBek 2002) and Urban Tapestries (Proboscis 2002-2008) employed location-based and mobile technologies to allow users to trace their movement through the city and "tag" or annotate the everyday urban environment with text and images. They also often explicitly appropriated the rhetoric and tactics of radical avant-garde groups like the Situationist International and their notion of the dérive and psychogeography (Zeffiro 2012: 254). They sought to deliberately critique the growing sense of alienation and disconnection from public life that the Situationists and theorists like Sennett and Virilio argue arose from twentieth-century urban development. According to Tuters and Varnelis,

since its inception ... locative media's practitioners have claimed an avantgarde position, insisting not only that their work is capable of creating a paradigmatic shift in the art world, but also that it can reconfigure our everyday life as well by renewing our sense of place in the world.

(2006: 358)

Location-based games in particular were at the forefront of this movement. As Drakopoulou writes, "at a time when projects in locative media were experimenting with cartography, location and situated interaction, location-based games were already at the forefront of research, using ideas of geo-mapping, tagging and ad hoc networks" (2010: 63). Like locative art, many location-based games sought to re-engage players with the public spaces of the city, reappropriating everyday urban locales as sites for competitive play and social interaction. 
De Souza e Silva and Hjorth (2009) and Flanagan (2009) point out that, like many locative artists, location-based game designers drew on a discourse of radical play in urban space, such as the figure of the flaneur and the Situationist practice of the dérive; avant-garde groups and movements like the SI, Archigram, and Fluxus; and contemporary urban practices like parkour. Locationbased games are seen as building on, and extending, these practices in the era of locative art and pervasive mobile media by bringing digital game play into the streets, parks, buildings, and public spaces of the city. De Souza e Silva and Hjorth (2009: 611) write, "like the flaneur/phoneur continuum, the practice of the dérive and the parkour, [location-based games] use city spaces as playful spaces."

Location-based games were typically designed by a small group of amateurs or a commercial developer employing a range of wireless and mobile technologies, such as GPS, Bluetooth, WiFi, Short Messaging Service (SMS), and mobile devices and networks. Early location-based games used mobile phones or specially designed headsets to allow players to share their location with each other and communicate while playing the game. More recent games, though, use the GPS technology embedded in smartphones to map and continually trace players' locations to create more sophisticated game scenarios. The goals of locationbased games vary widely: They might be simply to collect virtual items scattered around the city to score points like Mogi, Item Hunt (Newt Games, 2003-2007) or a cat-and-mouse style game such as BotFighters where players hunted each other across the cityscape. But they invariably all blend the virtual world of the game with physical space. Among the earliest and most influential iterations of these games were Alternate Reality Games (ARGs). The most widely known and pioneering ARG is The Beast, which was created in 2001 to promote the Steven Spielberg film A.I Artificial Intelligence. It was a kind of viral marketing game that invited players to participate in a plot set in the fictional world of the film and required them to follow cryptic clues through various media to progress through the story. Participants entered the world of the game through a cryptic line on the film's poster, which included a credit for a fictitious "sentient machine therapist." Curious fans eventually found a phone number for the "therapist," which turned out to be a recorded message providing just enough information to hook willing participants. As Bolish (2012) explains: "Through websites for fictional political groups, emails from in-film corporation, and phone conversations with characters, the project's creators immersed audiences in a complex game that blurred the line between real life and fiction."

The Beast subsequently paved the way for more ARGs that claimed to "blur" the physical and virtual worlds. But although some location-based games had their origins in marketing campaigns, many other projects emerged that involved small groups of people creating their own "interventions" into public spaces by appropriating them for play. These projects were conducted more in the vein of the locative art movement, albeit with more of a focus on competition and pointscoring, not unlike multiplayer digital games (de Souza e Silva and Hjorth 2009: 
618). One of the best-known examples is Pacmanhattan, which is essentially a real-life re-enactment of the classic arcade game Pac-Man (Namco 1980) using the streets of New York. One player takes on the role of Pac-Man-complete with a themed costume - who must navigate the streets of New York, while four other players are the "ghosts" who must chase and track him down. Each player is directed by a "controller," who tracks their movement on the game map by GPS and communicates their position and proximity to other players by talking to them on a mobile phone.

Other "real-world" and "hybrid reality" games like Alien Revolt (MIND Corporation, 2005-2007), Big Urban Game (BUG) (Lantz et al. 2003), Can You See Me Now? (Blast Theory 2001), Five Courts (KMA 2006) and Uncle Roy All Around You (Blast Theory 2003) all similarly sought to repurpose everyday locales and public spaces for play. ${ }^{1}$ Blast Theory's work in particular has its roots in theater and performative art (Dias 2012: 9-10), thereby seeking to challenge the distinction between performative art and digital games. Like many artistic performances, interventions, and "happenings" that took place throughout the twentieth century, location-based games seek to appropriate everyday spaces in the city for playful interactions. Location-based games, however, take advantage of the growing ubiquity of mobile communication devices and locative media to engage players of digital games with the environment, objects, and people around them in ways that echo or perhaps "remediate" these earlier art forms and practices (see Flanagan 2009: 197-200).

\section{Location-based games and the rise of the app economy}

Location-based games emerged from artistic and commercial projects that reappropriated locative media technologies, as well as experimental marketing campaigns like The Beast. Today, however, many location-based games are designed as apps for smartphones with much more accurate and sophisticated inbuilt GPS technology and advanced graphics engines. But more importantly, they are able to be distributed instantaneously to millions of users worldwide through digital distribution services like Apple's App Store (launched in July 2008) and Google Play for Android (launched in October 2008 as "Android Market" and rebranded in 2012 as Google Play). The rollout of the App Store and Android Market from 2008 onwards led to an explosion of mobile gaming development that transformed the mobile and handheld gaming market. Using development tools such as the iOS Software Development Kit (SDK) for Apple devices or Google's Android SDK, individual programmers and small teams of game designers are able to create games and upload them as apps on the App Store or Google Play. They can then be downloaded to the device for free or at a cost, with 70 percent of the revenue going to the developers and 30 percent to Apple or Google. These models thus allow less established, independent game designers to market their games to an ever-growing audience of iPhone, iPad and Android users at a 
significantly lower cost and shorter development cycle than is possible with the established console gaming market (see Cheng 2012; Feijóo 2012).

The release of the iPhone and Android and their respective app distribution services has similarly provided location-based games with a model that has enabled them to enter the mainstream mobile gaming market. Before the release of these devices, location-based games had no universal technology or platform that allowed them to reach a large audience. They typically utilized ad hoc, purpose-built devices like augmented-reality headsets, GPS-enabled handheld computers and computer scripts that were designed or retooled for a particular project. Otherwise, they simply appropriated a mix of readily available technologies like mobile phones, PDAs, and GPS-enabled devices. In this sense, as Farman (2012: 90-92) observes, location-based games could be described as a form of bricolage. Both their designers and their players repurposed already existing technologies for the project, often relying on whatever was available and suitable, or customizing or designing their own tools. In this sense, there was a kind of symbiotic relationship between the design of the game and the material constraints and limitations of the devices used, each informing the other. The devices provided designers and players of the game with certain parameters and limitations, since they were working with already existing technologies reappropriated or refitted according to their needs. But this is the nature of the bricoleur: to "make do" with whatever materials are available and adapt or rework them for the purposes of their project.

This bricoleur approach provided early location-based game designers with a mixed sense of freedom and contingency: They relied on self-organized groups of players or small commercial companies to assemble them in each separate city or region. For this reason, early location-based games never accomplished widespread popularity and recognition, nor were they able to generate anywhere near the levels of revenue as other genres of digital (or even mobile) gaming.

Of course, this is not to suggest that no initial location-based gaming projects were able to make a profit off their players. Games like Mogi, Item Hunt had a paid subscription model where users were charged a monthly fee to participate in the game's community. Similarly, artists such as Blast Theory were willing to embrace corporate funding and sponsorships to finance their projects (Tuters and Varnelis 2006: 360). Many location-based games also incurred incidental costs through mobile phone usage and SMS charges. In the case of BotFighters, which uses SMS to send data between players, the phone bills from players provided the game's main revenue source, which was shared between the game's developer, It's Alive, and Telia, a Swedish network operator (Dodson 2002). But in most cases, I would argue, any profits generated from the game were often merely a way of sustaining the project rather than necessarily to make substantial amounts of money from it. Because of their novel, rudimentary nature, these early projects relied on small groups of dedicated participants to make a profit and remain viable over a longer period of time. Similarly, there was no established model that their designers could rely on to make money and any revenue was likely only a 
means of supporting the project. There existed no established, universally accepted model for marketing and distributing location-based games, even though a considerable number of them were commercially funded.

With the release of the iPhone and Android platforms and their accompanying app stores, however, this has changed considerably. There are, of course, still location-based gaming projects being produced in particular cities and regionssome of them publicly funded or self-organized projects, others using the traditional subscription-based model of games like Mogi, Item Hunt. Most new location-based games, however, are designed as apps that can be purchased or downloaded free through digital distribution services like the App Store. These services provide location-based game designers with the ideal distribution model to sell or upload their game directly to any iPhone or Android device. As a result, location-based games have begun to move beyond their avant-garde, experimental origins and utilize the capabilities of these devices to become a mainstream genre in the mobile gaming market.

Location-based game designers are now able to exploit the much larger audience of users, wider geographic reach and advanced GPS technology and highend graphics that these devices afford. The penetration of smartphones- the iPhone in particular - into the mobile phone market has been one of the strongest forces enabling location-based games to appeal to a larger number of more diverse and geographically dispersed users. These devices are also typically carried everywhere by their users, meaning that they can be played in shorter, ongoing bursts of gameplay, and thus become more embedded in their everyday movement and lifestyle patterns. This is in stark contrast to the restricted number of players of location-based games like PacManhattan and Mogi, Item Hunt that were confined to particular cities or regions.

Location-based gaming apps remain a niche genre for the iPhone and Android in comparison with far more well-known and commercially successful games like Angry Birds (Rovio Interactive 2009). Nonetheless, dozens of location-based gaming apps have been released since the launch of the $3 \mathrm{G}$ iPhone in 2008 that have brought established (and some new) location-based game concepts into the mainstream. Seek 'n Spell was perhaps the earliest game released for iPhone that incorporated location-based technology as an integral part of the game. It is a spelling game developed by San Franciscobased app developer Dokogeo and released in 2009. In the game, players physically move around to collect virtual letters that are represented on the game screen on their iPhone; ideally, it is meant to be played in a large, open area such as a park or sports arena with several players. Like many early location-based games, the game explicitly emphasizes the fact that players are engaging with each other in physical space: the Google Play description of the game encourages players to "get outside and challenge your mind and body!" One review of the game prior to its release also praises the novelty of its use of GPS tracking technology, stating that it "will usher in a whole new genre in iPhone games" (Osborne 2009). 
It was not until 2011 and 2012, however, that location-based games began to receive more consumer and critical attention. These included the surveillancethemed game CodeRunner (Robot Chicken Interactive 2011); Shadow Cities (Grey Area 2011), praised in a review by the New York Times as "the future of mobile gaming" (Schiesel 2011); the "augmented sound" game Dimensions (Reality Jockey 2012); the fitness app Zombies, Run! (Six to Start 2012); the mafia themed Life is Crime (Red Robot Labs 2012); and Dokobots (Dokogeo 2012), designed by the same developers behind Seek 'n Spell. Also in 2012, Google released the beta version of their augmented reality multiplayer game for Android devices, Ingress, signaling that location-based gaming has perhaps at last become a mainstream, commonplace pastime for smartphone users.

\section{Location-based gaming apps: the next "killer app"?}

This shift marks an important stage in the evolution of location-based games, as they are increasingly developed as apps released in the highly competitive mobile gaming market, instead of artistic and commercial experiments on the periphery of the digital games industry. In contrast to early location-based games, which were predominantly documented through artistic and scholarly accounts of the projects, location-based gaming apps have been the subject of reviews and commentary from the videogame and technology industries. In the process, the language used to describe location-based games has become increasingly commercial and business-oriented, focusing on their appeal to consumers and potential to be commercially viable products within the mobile gaming market. An opinion article in the technology blog Mashable, "Why locationbased gaming is the next killer app," for instance, discusses the growing number of gaming apps using location-aware technology, advising that "marketers would do well to think about how these integrations can enhance the gaming experience" (Steen 2011). It goes on to claim:

The market is primed for the right game to galvanize interest in experiences that combine the real and virtual worlds. Just as FarmVille put social gaming on the map and Angry Birds brought attention to mobile gaming in general, we could see a wave of smartphone owners flood the application markets looking for similar experiences. This will present a valuable opportunity to marketers that want to foster emotional connections with their audiences.

The growing emphasis on location-based games as commercial products that "present a valuable opportunity to marketers" signals that they have well and truly entered the mainstream digital games industry - even if they still remain a niche genre within it. As a result, location-based game designers have begun to develop an economic structure and business model that contrasts sharply with 
the ad hoc assemblage of artists, amateurs and enthusiasts that created many early location-based games. A number of key game developers have begun to emerge that specialize in designing location-based gaming apps: the aforementioned Dokogeo (Seek 'n Spell, Dokobots), Red Robot Labs (Life is Crime, Life is Magic), and Grey Area (Shadow Cities) studios. The app economy has provided small start-ups like these with a financial model that allows them to compete alongside other mobile game developers for a share of the mobile industry's highly lucrative revenue stream - with varying degrees of success, of course.

But while app distribution services may have laid the foundations for location-based game designers to emerge as viable competitors in the mobile gaming industry, it has also meant that they must adapt to this new market structure. As Feijóo (2012: 86-88) notes, the emergence of new distribution platforms like the App Store and Google Play may have cut the costs of game development and facilitated the rise of small developers catering to the "long tail" of players with products aimed at niche interests. At the same time, though, it has paradoxically led to growing concentration and consolidation among opposing firms, as they seek to compete across multiple platforms and reach ever greater numbers of users.

Among location-based game app developers there have already been signs of this growing competitiveness and push for control over market share. In 2011, Red Robot Labs launched "the world's first location-based gamer network," named $R 2 . R 2$ is a proprietary network that provides designers willing to sign up with Red Robot with a platform to create location-based games using the software and network infrastructure developed by the company. According to a company press release, $R 2$ gives developers "unique access to its location-based expertise and publishing rights under Robot Labs"; "funding and/or revenue share options"; and access to the same back-end technology they use in their games, without the need for other developers to build location-based game engines from scratch. ${ }^{3}$ In 2012, the company announced that it has invested US\$2 million in signing up third-party developers to the network, as well as acquiring several small regional game development studios to expand their reach. Doing so allowed them to outsource their production to several smaller studios and, perhaps more importantly, boost their presence on the sales charts by having more games listed under their label (Woguem 2012).

Red Robot's launch of their $R 2$ platform for in-house and third-party development of location-based games suggests a growing consolidation and standardization of the genre. In some ways it simply replicates the business model of the traditional videogames industry, whereby one company owns the rights to a type of software called "middleware" (such as a graphics engine) that is then licensed out to smaller developers (Kerr 2006: 91); albeit on a micro-scale. The advantages of app distribution which has enabled location-based games to enter the mainstream market-decreased costs, a direct-to-consumer publishing model, niche products aimed at the "long tail" - are thus being counterbalanced by 
economies of scale and growing competition among developers at the bottom of the supply chain.

As a result, some of these once modestly sized independent studios have evolved into formidable corporations, while others are simply swallowed up or attach themselves to the dominant companies' established publishing model in order to subsist. In 2011, for instance, the social gaming company Zynga acquired Area/Code, a New York-based studio co-founded by Kevin Slavin and Frank Lantz, the latter of whom helped design formative location-based and realworld games like PacManhattan and BUG (Big Urban Game). As the examples of Red Robot and Zynga illustrate, then, location-based game designers are no longer impervious to the broader market forces of the digital games industry, as they become increasingly recognized by developers and investors as a potentially lucrative business investment.

\section{Life is Magic: location-based games and the labor of play}

Not long after heavily investing in their R2 proprietary network and signing up - or acquiring - a number of location-based game design studios, Red Robot released their next major game for iPhone, iPad, and Android, titled Life is Magic (2013). It builds on the formula of their 2012 game Life is Crime, in which players take on the role of the leader of a criminal gang to commit "virtual crimes" by taking over real estate in their local neighborhood to become "the most notorious criminal where you live." ${ }^{4}$ In Life is Magic, however, the player's local neighborhood appears in the game as a fantasy world inhabited by monsters and characters who provide them with quests, spells, and weapon and armor upgrades. It is perhaps the first location-based game where real, everyday locations are "re-skinned" and rendered by the game's engine as virtual objects: Buildings and houses appear as medieval shops, inns, and towers, while streets and public spaces are depicted as dirt paths and fields. As a result, the game plays much like a standard turn-based fantasy RPG (role-playing game), with the added element that players are interacting with a parallel world that resembles their own, allowing for location-based play.

While Life is Magic emulates the design and rhetoric of earlier location-based games, players do not physically interact with the objects, people, and surroundings around them as part of the game. Instead, they engage with them solely through the interface of the game on their device, which consists of a map of their local area rendered through the game's graphics engine. This is also the case for many other location-based gaming apps, including Life is Crime and Shadow Cities; the latter was one of the first mainstream, popular location-based games released for iPhone (see Schiesel 2011). However, it lasted only three years: the game's sever was closed down late in 2013 and the game was pulled from the Apple Store. ${ }^{5}$ In Life is Magic, the player's local region or country appears geographically the same on the game's map as in the "real world," except that it is 
rendered in a cartoonish style and is populated with mountains, forests, and various types of "dungeons" (which players can enter to defeat monsters and level up their character) in place of the usual topography. Major cities also appear as "towers," which can be captured by challenging the bosses that reside there. In addition to this "regional" level of the map interface, players can also zoom in to their neighborhood (which depicts an area of about four or five city blocks). This level is rendered in more detail: Local shops, buildings and landmarks (complete with their real-world names) serve as the in-game stores and meeting areas where players can acquire quests and items, and information about the game. It always resembles the nearby area the player is located at using GPS information, so if they live outside a town or city they will have to purchase in-game "travel tokens" to take them to a nearby city to be able to access all these features of the game.

Like most RPGs, the goal of Life is Magic is to level up your character and earn enough in-game currency that you can acquire new skills and abilities and open up new areas of the game. In the game, this means finding gold which is "dropped" by defeated enemies and is used to purchase new weapons, armor, and healing potions to improve your chances in battle. To upgrade most of your character's specific skills or spells that are used in battles, though, you need to acquire much rarer "crystals" which are only awarded for more difficult tasks like finishing a quest or defeating the boss of a dungeon; tasks that require a substantial amount of skill and/or time investment. In addition, to enter any of the dungeons required to progress through the game, players need to use "influence," which is represented by a finite counter that restores slowly over time. Lastly, in the spirit of games like World of Warcraft, one can also add other nearby players to one's "party," allowing them to help out with difficult fights. While these are other real people who happen to be playing the game in the same neighborhood or city, there is no simultaneous or co-present play involved. Instead, that person's character simply appears in one's team and-since battles operate on a turn-based system - one chooses that character's moves for them when their turn comes, regardless of whether their owner is playing at the same time or not. Likewise, they can use the player's own character in their game.

Life is Magic builds on the legacy of earlier, experimental location-based games that emerged during the locative art movement by promising to engage players with their local neighborhood or city for playful interaction with one another. But where these games typically involved some level of physical, dynamic, and embodied interaction with the environment around them, Life is Magic reduces the location-based element of game play to a banal, abstract interaction between the player and the spaces and people around them. Players do not interact directly with the environment in which they play the game; instead it appears as a virtual map on their phone or tablet screen that merely serves as the template on which the game is played. Players can team up with other nearby players, but they can only do so asynchronously and the only way to communicate with them is by using the game's inbuilt chat room or by sending trivial "gifts" (usually healing items, weapons, or armor). Players can challenge 
each other to real-time battles, but the only purpose of this is scoring points or expanding one's influence in an area; things which are important in the context of the game, but arguably do not create "meaningful" encounters among players outside the virtual world of the game.

In addition to its reductive use of location-based elements in the game, Life is Magic operates under a commercial model which encourages players to spend real money on in-game items. While the game is free to download and play, it also allows players to make in-app purchases of items necessary to progress through the game. This feature was first introduced to location-based games in Shadow Cities, which also used a "freemium" model to draw players in while deliberately making the game laborious to play without spending real money to level up your character. Similarly, Life is Magic is made substantially more difficult and frustrating to play without occasionally spending real money to boost your stock of gold to purchase new healing potions or equipment. Likewise, influence (which is necessary to enter each level of the game's dungeons) is also often depleted after only an hour or so of playing the game and regenerates slowly over time, making it much easier to simply "top it up" for a few dollars in order to keep playing. The most prized items in the game, crystals, are also exceptionally rare; the only way to earn enough to upgrade your character to a reasonable level is by buying them up in large quantities through in-app purchases, unless players are willing to invest hours "grinding" and completing tedious quests to unlock them one at a time. Their cost ranges from AU\$5.49 for 50 crystals to AU\$109.99 (approx. US\$112) for 1,500. Crystals are also necessary to buy "travel tokens" which allow players to warp to other cities around the world; this is essential if the player happens to live in an area with few players or no nearby buildings or landmarks.

As McCrea (2011) points out, the digital distribution model of the app economy has led to a proliferation of cheap, on-the-go mobile games that are often released as free downloads and generate a profit through "microtransactions": a strategy pioneered by social networking games like FarmVille (Zynga 2009). As a result, these games are able to generate revenue for their developers by playing on exactly the desire for accomplishment and gratification that makes digital games so compelling. Their players are more often willing to spend real money to send virtual gifts to friends in FarmVille, avoid grinding for the materials necessary to progress through the game, or buy power-ups that allow them to pass a particularly challenging level. Microtransactions thus go hand-in-hand with the style of gameplay afforded by mobile games. As McCrea (2012: 393) notes, they are typically played on smartphones whenever their users are able to snatch a moment or two for short but intensive gaming sessions, usually of games like Angry Birds or Jetpack Joyride (Halfbrick Studios 2011) for casual players.

The incorporation of the "freemium" model into location-based games such as Life is Magic and Shadow Cities presents these games with a more commercially successful and sustainable revenue stream. But it has also meant that they have been assimilated within the "work as play" ethos that characterizes the contemporary digital games industry. They embody the way in which digital game 
play has arguably become a form of increasingly empty, instrumental labor in which players perform repetitive tasks or spend real money to advance in the game, thereby conflating the boundaries between work and play (see DyerWitheford and de Peuter 2009; Kücklich 2005; Wark 2007). Dyer-Witheford and de Peuter (2009: 93) argue that

To become a [videogame] player is to plug oneself into a network of technohuman relations, which even as it offers cognitive skills and affective thrills also inserts subjects into a commodity web involving not just the console purchase but that of the subsequent game software, the online subscriptions, the music and video services, and a whole branded identity built around gamer tags, achievement points, and the transfer of avatars, a grid of machinic coordinates engineered to the tolerances of corporate profit.

As location-based games enter into the value chain of the digital games economy through the app market, they too become entangled in this "network of technohuman relations." In the process, the location-based element of these games, as demonstrated by the gameplay of Life is Magic, becomes subordinated to the profit-driven, commercial aspect of the game. Instead of engaging their players with the people and spaces around them, gameplay in Shadow Cities and Life is Magic takes place entirely at the level of the game's interface. This interface serves as a net for capturing the labor performed by players - in leveling up their character, progressing through dungeons and acquiring items - and transforming it into a profit for the game's creators in the form of money spent on in-app purchases, advertising, and users' data. ${ }^{6}$ This is not to claim that this is a one-way process - undoubtedly players of these games gain a sense of accomplishment and pleasure in the process of playing it. But in contrast to earlier location-based games, where commercial profit was unlikely to impact significantly on gameplay, location-based gaming apps are centered entirely around this commercial logic. They seek to immerse players in the virtual interface of the game and encourage them to spend real money on leveling up their digital avatar, rather than interacting with the physical space in which the game is played.

There are still location-based gaming projects that challenge this commercialization by bringing play into social and physical spaces without being mediated through smartphones and other "closed" devices. A simple example is the web browser game GeoGuessr, which places the player in a random location anywhere in the world through Google's Street View interface. They must then explore their surroundings by clicking on the navigational arrows that appear in Street View before guessing where they are by clicking on a map of the world; points are awarded based on how close they are to their actual location. ${ }^{7}$ GeoGuessr is not strictly location-based in that it does not take into account the player's location. Nonetheless, the game is a clever inversion of the principle of location-based games: It encourages players to mobilize their knowledge of various cultures, languages, landmarks, and geographies from around the world 
to try and guess which country (or even continent) they find themselves in. A more radical example of a recent non-commercial location-based game is Camover 2013, which emerged as a response to the growing number of surveillance cameras appearing in Berlin. Participants of the game are awarded points for destroying CCTV cameras around the city and uploading videos of their exploits on the project's website, with bonus points given for especially inventive methods of destruction. ${ }^{8}$

Projects like these illustrate that there still remains a space for location-based games to critique dominant ideologies and assumptions (as well as the laws) of public spaces and everyday urban locales. Nonetheless, the emergence of the app market has brought about a palpable shift in the design of location-based games that is already reshaping the economic structure of the industry and the games currently being produced. On one level, this is a positive development for the creators of these games. It means that they can potentially be exposed to a wider audience of players and generate a greater diversity of engagement with public space through more sophisticated gaming interfaces. But as Red Robot's $R 2$ network and the growing competition among the various location-based game development studios illustrates, these commercial imperatives have instead begun to push location-based games more toward generic, standardized formats that can be endlessly replicated and reproduced.

As location-based games become increasingly integrated into the mainstream mobile games industry, this becomes the overriding motive of games designed for platforms like the iOS and Android: to produce more sophisticated ways of capturing players' leisure time, money and data at the expense of meaningful interaction with embodied space. But nor does it seem as if this model is necessarily sustainable in the long term. Despite the rhetoric about location-based games being the next "killer app" (Steen 2011), no location-based games have made a significant impact in the mobiles games level on the same level as something like Angry Birds. And, as mentioned above, Shadow Cities-the locationbased game app that has received the most critical attention-was closed down after three years. Likewise, as of 2014 it appears that Life is Magic has also been closed down; it is no longer available for download on Apple or Google's app stores. The future of location-based games, then, has become entangled within the highly competitive and economically precarious conditions of the app economy that initially catapulted it into the mainstream digital games industry.

\section{Conclusion}

In this chapter, I have drawn on the case study of Red Robot Labs' game Life is Magic, as well as a range of location-based gaming apps that have been released for the iOS and Android platforms over a number of years, to illustrate the growing commercialization of location-based games in the app economy. Of course, Red Robot is only one location-based game developer currently making games for these platforms, and like any commercial game developer they also have to meet 
their investors' and stakeholders' expectations. There also remains the question of whether this process is necessarily a negative development for location-based games; after all, it illustrates a natural progression as location-based games evolve from a niche genre into a recognized, mainstream videogame format. The commercialization of location-based games within the app economy reflects a broader trajectory whereby locative media technologies - once the purview of artists and amateurs - have been subsumed within the corporate infrastructure of technology giants like Apple and Google. As Varnelis (2011) writes:

Locative media remained the stuff of demos and art-technology festivals until 2008 when Apple released the GPS-enabled iPhone 3G. Paradoxically, the mass realization of locative media seems to have taken the wind out of its sails as an art form. Although courses on writing apps proliferate in art and architecture programmes, the promise of locative media seems to remain just that: a promise.

Early location-based games such as PacManhattan, BotFighters, and Mogi were similarly products of their particular "moment" - one characterized by experimentation with and repurposing of novel location-aware devices and technologies that, at the time, had yet to find any widespread commercial or mainstream application. Today, though, the emergence of location-based gaming apps signals a significant shift from early location-based games' emphasis on engagement with public space, which as I have argued is closely aligned with the politics of the locative art movement. All location-based gaming projects must deal with the unique characteristic of the cities in which they take place, and many require a commercial model to profit from their players. In the case of games like Red Robot's Life is Magic, however, these issues become mobilized on a much larger scale. These games uncritically appropriate the concept of locationbased gaming within the context of the emerging app economy. They often utilize a "freemium" model that focuses gameplay around purchasing in-game content and accomplishing tasks that fit within the game's commercial logic. In turn, this leads location-based gaming toward a greater degree of commodification which privileges the game's technical interface-as a tool for generating profit - over engagement with other players and lived, embodied spaces through the experience of playing the game. As a result, location-based gaming risks becoming simply another generic form of digital gameplay that reflects the growing trend toward commodification and the conflation of work and play currently taking place within the videogame industry more broadly.

\section{Notes}

1 For a more detailed taxonomy of the various genres and types of location-based games, see de Souza e Silva and Hjorth 2009; Drakopoulou 2010; Montola et al. 2009; von Borries et al. 2007. 
2 Source: https://play.google.com/store/apps/details?id=com.retronyms.android.seeknspell_ heavy.

3 Source: www.marketwire.com/press-release/red-robot-labs-signs-new-3rd-party-developersto-its-r2-mobile-geo-gaming-platform-1693385.htm.

4 Source: http://redrobot.com/games/life-is-crime/.

5 Source: http://massively.joystiq.com/2013/09/20/shadow-cities-closing-down-october-7.

6 Indeed, concerns have already been raised over Google's use of data generated by users of their augmented reality game Ingress; see Hodson 2012.

$7 \mathrm{http}: / /$ geoguessr.com/.

8 Source: www.theguardian.com/theguardian/shortcuts/2013/jan/25/game-destroy-cctvcameras-berlin. For the project's website, visit: http://camover.noblogs.org/.

\section{References}

Augé, M. (1995) Non-Places: Introduction to an Anthropology of Supermodernity (trans. John Howe), New York, NY: Basic Books.

Bolish, M. (2012) Convergence: Down the rabbit hole with "The Beast," Film Society Lincoln Center, available at: www.filmlinc.com/blog/entry/convergence-down-therabbit-hole-with-the-beast.

Cheng, C. (2012) The system and self-reference of the app economy: The case of Angry Birds, Westminster Papers in Communication and Culture, 9(1): 47-66.

de Souza e Silva, A. and J. Frith (2012) Mobile Interfaces in Public Spaces: Locational Privacy, Control, and Urban Sociability, New York, NY/Abingdon: Routledge.

de Souza e Silva, A. and L. Hjorth (2009) Playful urban spaces: A historical approach to mobile games, Simulation \& Gaming, 40(5): 602-625.

Dias, M. (2012) A machine to see with (and reflect upon): Interview with Blast Theory artists Matt Adams and Nick Tandavanitj, Liminalities: Journal of Performance Studies 8(1), available at: http://liminalities.net/8-1/blast-theory.pdf.

Dodson, S. (2002) Ready, aim, text, Guardian, August 15, available at: www.guardian. co.uk/technology/2002/aug/15/electronicgoods.games.

Drakopoulou, S. (2010) A moment of experimentation: Spatial practice and representation of space as narrative elements in location-based games, Aether: Journal of Media Geography, 5A: 63-76.

Dyer-Witheford, N. and G. de Peuter (2009) Games of Empire: Global Capitalism and Video Games, Minneapolis, MN: University of Minnesota Press.

Farman, J. (2012) Mobile Interface Theory: Embodied Space and Locative Media, Abingdon: Routledge.

Feijóo, C. (2012) An exploration of the mobile gaming ecosystem from developers' perspective, in P. Zackariasson and T. L. Wilson (eds.), The Video Game Industry: Formation, Present State, and Future, New York, NY: Routledge, pp. 76-95.

Flanagan, M. (2009) Critical Play: Radical Game Design, Cambridge, MA: MIT Press.

Gordon, E. and de Souza e Silva, A. (2011) Net Locality: Why Location Matters in a Networked World, Malden, MA: Blackwell.

Hemment, D. (2004) The Locative Dystopia, Nettime, available at: http://amsterdam. nettime.org/Lists-Archives/nettime-1-0401/msg00021.html.

Hemment, D. (2006) Locative arts, Leonardo, 39(4): 348-355.

Hodson, H. (2012) Why Google's ingress is a data gold mine, New Scientist, 2893: 19, 
available at: www.newscientist.com/article/mg21628936.200-why-googles-ingressgame-is-a-data-gold-mine.html.

Ito, M. (2005) Introduction: Portable, personal, pedestrian, in M. Ito, D. Okabe, and M. Matsuda (eds.), Personal, Portable, Pedestrian: Mobile Phones in Japanese Life, Cambridge, MA: MIT Press, pp. 1-18.

Kerr, A. (2006) The Business and Culture of Digital Games: Gamework/Gameplay, London: Sage.

Kluitenberg, E. (2006) The network of waves: Living and acting in a hybrid space, Open, 11: 6-16, available at: www.skor.nl/eng/news/item/open-11-hybrid-space-how-wirelessmedia-are-mobilizing-public-space?single $=1$.

Kücklich, J. (2005) Precarious playbour: Modders and the digital games industry, Fibreculture Journal, 5, available at: http://five.fibreculturejournal.org/fcj-025-precariousplaybour-modders-and-the-digital-games-industry.

McCrea, C. (2011) We play in public: The nature and context of portable gaming systems, Convergence, 17(4): 389-403.

Montola, M., J. Stenros, and A. Waern (2009) Pervasive Games: Theory and Design, Burlington, MA: Morgan Kaufmann.

Osborne, B. (2009) Seek 'n Spell to Soon Launch a GPS-Based iPhone Game, Geek.com, available at: www.geek.com/articles/mobile/seek-n-spell-to-soon-launch-as-gps-basediphone-game-20090312/.

Rheingold, H. (2002) Smart Mobs: The Next Social Revolution, Cambridge, MA: MIT Press.

Schiesel, S. (2011) Brave New World that's as familiar as the machine it fights with, New York Times, July 16, available at: www.nytimes.com/2011/07/16/arts/video-games/ shadow-cities-a-new-iphone-video-game-review.html.

Sennett, R. (2002 [1977]), The Fall of Public Man, London: Penguin Books.

Sorkin, M. (1992) Introduction: Variations on a theme park, in M. Sorkin (ed.), Variations on a Theme Park: The New American City and the End of Public Space, New York, NY: Hill and Wang, pp. xi-xv.

Steen, G. (2011) Why location-based gaming is the next killer app, Mashable, available at: http://mashable.com/2011/07/24/location-based-gaming/.

Tuters, M. and K. Varnelis (2006) Beyond locative media: Giving shape to the internet of things, Leonardo, 39(4): 357-363.

Varnelis, K. (2011) The rise and fall of new media in the art world, Frieze, 141, available at: http://frieze.com/issue/article/down-the-line.

Varnelis, K. and A. Friedberg (2008) Place: The networking of public space, in K. Varnelis (ed.), Networked Publics, Cambridge, MA: MIT Press, pp. 15-42.

Virilio, P. (1997 [1984]) The overexposed city, in N. Leach (ed.), Rethinking Architecture: A reader in Cultural Theory, New York, NY: Routledge, pp. 381-390.

Von Borries, F., S. P. Walz, and M. Bottger (eds.) (2007) Space Time Play: Computer Games, Architecture and Urbanism: The Next Level, Basel: Birkhauser.

Wark, M. (2007) Gamer Theory, Cambridge, MA: Harvard University Press.

Woguem, D. (2012) Red Robot Lab upsizes its location-based gaming platform, R2. Geo Social Games, available at: http://geosocialgames.com/red-robot-lab-upsizes-itsmobile-location-based-gaming-platform-r2.

Zeffiro, A. (2012) A location of one's own: A genealogy of locative media, Convergence: The International Journal of Research into New Media Technologies, 18(3): 249-266. 


\section{Chapter 9}

\section{Houses in motion}

\section{An overview of gamification in the context of mobile interfaces}

Nathan Hulsey

Mobile advertising and location-aware applications are slowly becoming synchronized to the rhythms of urban life (Cronin 2006; Wilken and Sinclair 2009). At the same time, gaming and mobile devices have come to the forefront as a new brand of playful mobility that creates unique dynamics of spatial practice (Chan 2008; Gazzard 2011; Licoppe 2009). By examining the concept of gamification and the subsequent embedding of game mechanics into everyday activities such as shopping, mobile practice, energy consumption, and spatial representation, I broaden the conversation about the place of games and play into the context of mobilities.

Scholarly work tracking developments in mobile communications and ubiquitous computing have noted a certain affinity between game mechanics and spatial practice (Sutko and de Souza e Silva 2008). Pervasive games such as Blast Theory's Can You See Me Now? and Uncle Roy All Around You use mobile technology to highlight the interplay between physical environments, technology, and play (de Souza e Silva and Hjorth 2009; Montola et al. 2009; Benford and Giannachi 2011). Pervasive games are commonly defined as games that break with the traditional boundaries (the screen, a board, a device) and expand gameplay socially, spatially, or temporally (Flintham et al. 2007; Stenros et al. 2012; Montola et al. 2009). In the case of pervasive games, mobile technologies and applications are an intrinsic part of a playful mobility that is utilized to influence playful behavior. The penetration of mobile devices has brought pervasive gaming out of the realm of experimentation and into the mainstream, especially in the case of marketing and advergaming (Campbell 2011; Sennott 2005). Mobile marketing tactics that include game-like qualities differentiate themselves from pervasive games by emphasizing the behavioral results of ludic, or game-related, protocols such as mechanics over the production of a self-contained, playful environment. In other words, there is a growing trend for applications to include pervasiveness and playfulness without ever creating (or marketing) a game. This method of design, known as gamification, seeks to incorporate game-like qualities into non-gaming systems and applications (Swan 2012; Scofidio 2012; Savitz and Heisler 2012; Liyakasa 2012b, 2012a). Mainstream gamified applications such as McDonald's Monopoly, an interactive 
consumer rewards service, and Shopkick, a location-based shopping app, use varying forms of game mechanics while never actually making themselves out to be "a game." Rather, gamified mobile applications act as mobile interfaces that attempt to induce compulsive behaviors in the user, motivating them to engage with a system of commodification that is then monitored in real time by the designers of the application.

I argue that gamified mobile applications help constitute hybrid spaces, where data, bodies, perception, and physical environments are inextricable from one another (Benford and Giannachi 2011; de Souza e Silva 2006). However, my line of enquiry slightly departs from examining the changing notions of spatiality that hybrid spaces encapsulate in favor of examining the downside of exploiting the synergy between mobile technologies and game mechanics for synoptic and consumerist aims. In short, game mechanics and mobile interfaces work in concert with each other to recontextualize physical and social spaces while also allowing for profitable lateral and top-down surveillance. Using applications such as McDonald's Monopoly, an interactive consumer rewards service; Shopkick, a location-based shopping app; and Green Button, a domestic energy management application as examples, I show that gamified mobile interfaces produce frames, understood as the material, social, and ideological boundaries that precede territory. These frames subsequently help to influence the spatial practices of users, especially in regard to leisure, shopping, and consumption. First I explore how gamification operates in a mobilities context by exploring consumerist applications. Second, I examine how gamification is implicit in the creation of hybrid spaces by examining gamification in a domestic context. Each of these examples serves to highlight an increasing trend involving the use of game mechanics as a vehicle for the surveillance-driven colonization of spaces and practices that previously either could not be monitored or, on the other hand, encountered strong popular resistance to monitoring (i.e., the home). Key here is the idea that introducing motivational protocols makes users more willing to engage in systems that track their location and their behavior under the guise of play. By exploring consumerist and domestic examples, this chapter adds to previous literature (de Souza e Silva and Frith 2012) concerning the interactions between hybrid space, mobile practice, and surveillance.

\section{Monopolizing the city: gamification and mobilities}

Gamification is a process that seeks to add game mechanics to business, social, and political transactions. Many gamified applications are similar to "serious games" (Bogost 2007) that produce "meaningful play" (Murff 2011): They utilize location-aware devices like smartphones and built city environments to enable gameplay and they have intent and purpose beyond intrinsic gameplay. I contend that there are also distinct differences between so-called serious games and gamification. Rather than seeking to create a self-contained game, gamification seeks to make consumption viral and increase engagement with products, 
tasks, locations, and services (Zicherman and Linder 2010). In this way, it is pervasive; however, unlike experimental art, gamification is also commercial, behaviorist, and surveillance-oriented. Gamification is most often associated with corporations and marketing. In a recent article, The Economist noted that games and gaming have become more visible than ever in the business sector (2011). It is important to note the concept of gamification is not new. Frequent flier miles, punch cards, and customer loyalty programs like credit card "cash back" programs have been around for a long time. Role-playing and simulations have also frequently been used in education and organizational training and for business purposes (Lawson and Lawson 2010; Kriz 2009; Dieckmann et al. 2012). Designers of gamified systems use the practice of layering game mechanics and play-based initiatives to make certain tasks, products, and services seem like a game. Game mechanics can be described as rule systems and virtual currencies that encourage certain cooperative or competitive behaviors in players (Sicart 2008). For instance, rewards, badges, leaderboards, and specials unlocked through dedicated interaction with the game's system of rules are types of currencies. Another important aspect of virtual currency is progression and accomplishment through cascading information, levels, and quests.

Trends in gamification literature from business publications have centered on the social nature of games in combination with the surveillance mechanisms of data mining. The social-mobile gaming revenue from advertising alone was US\$368 million in 2011, and is expected to rise to US\$672, nearly doubling over the course of a year (Delo 2012). Marketers and business communicators have taken note of the ability of games to increase engagement with a product; they have also noted the ability to track customer use and increased consumer feedback (Zicherman and Linder 2010; Zuk 2012). "Human techsourcing" is the concept that technology can "convert" humans into living network connections. The idea is that social-mobile initiatives and games not only tap into the groupthink mentality, but also aid in crowdsourcing information and gathering data on human activities by building motivational apparatuses aimed at encouraging information-sharing into applications (Jacobs 2012). For these reasons, the term is already receiving backlash from some members of the business community. Gamification is being described as "Skinnerian" and a "black box" approach centered on control and surveillance (ASTD Staff 2012).

It is important to note that gamification is not just for training, conditioning, and surveillance. It is also praised for its ability to induce "good" behaviors in individuals and motivate large masses to engage in actions beneficial to a larger cause. For example, in 2008 the University of Washington gamified their research initiative to recognize the combinations of protein folds. Poorly folded proteins are linked to diseases like Parkinson's, but there are trillions of combinations. A computer can recognize a "bad" fold, but there was no way a small research team could configure all the possible combinations without access to a supercomputer. So, they created Foldit, an online puzzle game that turns the collective intelligence of users into a (free) supercomputer. Foldit and its 57,000 
users have already made major contributions to molecular biology (Ehrenberg 2010). Also, efforts at gamifying smart-energy meters and recycling programs have produced significant results (Austin 2014; Massey 2012). Still, the majority of applications are inevitably aimed at mining everyday activities for new modes of capital.

\section{Mobility and gamification}

In the context of mobile applications, many credit McDonald's as a pioneer in location-based gamification with its Monopoly promotion, which encourages customer loyalty and consumption through gamifying its food packaging with color-coded tabs. McDonald's Monopoly only resembles the board game in basic form: it involves collecting color-coded properties. However, in order to obtain property pieces, the user usually must make some kind of purchase. The game pieces are thus spatially spread over various McDonalds locations. By searching for matching colors through purchases, players can win prizes (Zuk 2012). The game was immensely popular and has been played seasonally for years since its inception in 1987. However, it is the more modern iteration that drew the interest of mobile application designers. McDonald's Monopoly is perhaps the first instance of gamifying consumption where the spatial practice of consumers has been altered significantly. McDonald's has added social media and mobile applications; in addition, they have explored the idea of allowing players to track winning "hotspots" and plan routes around their places of residence to track their play. Through implementation of mobile, location-based technology into the marketing scheme, consumers could be encouraged to alter their routes in order to hit the maximum number of McDonald's stores. Consumers often bought a superfluous amount of supersized drinks in order to get the play pieces. As a result, total profits in the US rose 5.6 percent during the play period (Chief Marketer 2011). The associated website, where users (and McDonald's) could track play and movements, received nearly five million unique hits in forty-two days.

By gamifying fast food consumption, McDonald's invited users to attach new meanings to the spaces in which they live and traverse. The space of the city, and the locations where virtual currencies could be acquired, effectively became a grid for play (Licoppe and Inada 2008, 2010; de Souza e Silva 2008). In this manner, one could argue that the space of the city, through the use of playful mobile technology, becomes recontextualized. This recontextualization plays a role in what Urry (1995) describes as "the consumption of places," in which space, goods, leisure, and consumptive practice intertwine in a manner that creates a complex set of interdependencies. Spatial frames are reset by the protocol and mechanics of play, which are in turn dictated by a corporate or governmental entity. McDonald's. Monopoly is not restricted to a cardboard square and fake money, and it does not end when a player dominates his or her opponents. Instead, the nature of leisure, as Baudrillard (1998) suggests, becomes converted into a broader system of capital where leisure is no longer a "free" waste 
of time, but rather a productive activity linked to forces of controlled consumption.

Commodification of movements through space creates an environment that attaches currency to physical movements. Popular applications that spatialize social networks and consumptive practice such as Foursquare utilize game mechanics and social/spatial relations to extend into the realm of everyday activities while also channeling a desire for conspicuous mobility (Wilson 2012). Wilson states conspicuous mobility is linked to generative modes of capital, as well as surveillance:

applications like Foursquare ... allow users to "check-in" at restaurants, bars, gyms, retail outlets, and offices, thereby sharing their location within their social network. These developments enable consumers to (re)discover their proximities to products, while feeding a desire for making known one's everyday movements

(Wilson 2012: 1266)

In this context gameplay can either enable or hinder movements by generating different modes of currency in different spatial and social contexts, for instance, encouraging movements to only certain stores in the case of Monopoly. Another way in which movement is commodified is through silence, or the empty areas where spatial representations such as maps conceal or leave out information (Harley 2001). In this context, withholding currency and spatial information commodifies representational spaces by making no movement a "free" movement in the sense that users can chart their own paths without implicit penalties.

\section{Gamification and mobile technologies}

Game mechanics, real-time surveillance and mobile technologies combine with successful design practices to make gamification a powerful tool for influencing behavior. The purpose of game mechanics and game design is to "drive" behavior through motivation and diminishing rewards - also known as partial reinforcement (Loftus and Loftus 1983). Yet gamification is not about games or even play; it is the process of applying certain game mechanics to everyday situations solely to produce behavioral results. This difference may seem slight at first, but the implications are relatively far-reaching. Zicherman and Cunningham (2011), in their book Gamification by Design, argue that gamifying is a two-pronged process. On one hand, it is about controlling the behavior of customers by increasing "engagement," or repetitive action, with certain people, products, and locations through play. On the other hand, it utilizes play data as a form of surveillance. The social environment in which gamified systems operate accepts the existence of surveillance as necessary or useful. This is related to the concept of the digital enclosure, or a system of ubiquitous computing that touts creativity, ease of use, and connectivity on the surface while tacitly promoting 
the interests of governments and advertisers through data collection (Andrejevic 2006, 2007). Gamification, because mobile marketers and corporate investors typically employ it, does not currently serve as a site that encourages intervention or critical reflection on the current state of the user's position in the digital enclosure. Rather, gamified systems often operate explicitly to smokescreen surveillance protocol by drawing attention toward game mechanics.

For example, projects that use pervasive gaming promote a critical intervention in conceptualizing surveillance and mobile technologies differ from gamified applications. Sutko and de Souza e Silva (2008) state that playful aspects of culture are inextricably intertwined with daily practices through pervasive games. The authors also view pervasive games as indicative of how space, technology, and notions of surveillance correlate with the concept of hybrid space, or a space in which social, virtual, physical aspects are simultaneously embedded. This is certainly true of many location-aware applications. However, the key differences between gamified mobile applications and experimental pervasive games lie in the definition of a game: Gamification has everything to do with playfulness outside of games. For instance, Can You See Me Now? created a bounded-off gamespace within the city. The surveillance in Can You See Me Now? was artistic: Users on a computer had to watch and direct users on the street as part of the game. The purpose was to promote critical thinking on how urban space, sociability, and technology are increasingly entwined. As art, Can You See Me Now? began and ended with clear boundaries: The gameplay and gamespace were self-contained and the rewards for play were intrinsic. Commercial applications of location-based games such as BotFighters (de Souza e Silva and Hjorth 2009) and Mogi (Licoppe and Inada 2008) differ only slightly_while these games certainly were not aimed at critical intervention, their use was intrinsic to the logic of spatial gameplay as divested from abject commodification. That is to say, they were not employed as motivational apparatuses aimed at promoting or sustaining wider grids of consumptive practice divested from the games themselves. Using an application like Shopkick is far different. No defined gamespace exists and there is no beginning or end to play. Surveillance in apps like Shopkick and Monopoly is not an artistic statement; it produces cold hard capital.

For example, Shopkick, a location-based app that seeks to track and influence shopping behavior, introduces "bubbles" which users must "pop" by walking into designated store locations. Popping bubbles (a game mechanic) gets the user "kicks," or a point reward, which can be cashed in for various prizes and coupons. By adding a virtual layer of currency and playful mechanics to business and social transactions, corporate entities can harness the cognitive will-toplay. Users of Shopkick alter their daily routine and spatial practice in order to accumulate points, which may or may not result in tangible rewards (it depends on the amount of time spent engaged with the system). At the same time, this system also generates valuable "walk-ins" for stores, which also get feedback on their products and store layout from the application's three million users (Crunchbase 2011; Rao 2012b). All of this is gained through a seemingly simple 
combination of game mechanics, cell phones, and positive reinforcement. Another app that works similarly is Waze, purchased by Google in 2013 (Wasserman 2013), which uses a location-aware rewards system to encourage user-submitted traffic information in real time (Lopez 2012). Both applications rely on the location of the user and social interaction to record titles and rewards and thus require mobile technology and related infrastructure to function. Despite these variations, I contend that the concept of hybrid space (de Souza e Silva 2006) is still a key component of gamified mobile applications.

\section{The technique of gamification}

By definition gamification as technique entails (1) utilizing parts of a game- the mechanics, rules, and rewards - to increase the predictive capabilities and/or efficiency of much larger systems; (2) promoting repeated non-coerced engagement within a set protocol for an end result outside of the gameplay itself; (3) surveilling player actions for the profit of the designer and the continuation of engagement with the system; and (4) tapping into the business of leisure and pleasure through harnessing playful desires.

Shopkick started out as a simple way of finding local shopping centers and offering a locative-based coupon/advertising service to dissuade users from "showroom behavior" where they would look at objects in a store and then buy them later online (Crunchbase 2011). The differences between Shopkick and a traditional game can be related to intrinsic and extrinsic rewards. Traditional game rewards can broadly be described as intrinsic, or limited to the game or system itself. Shopkick's reward system is extrinsic because players are motivated by consumerist rewards that can be applied "in real life." Also, users of Shopkick will never "beat" it. The mechanics of Shopkick are there to ensure continued play with no winner. Through combining these elements of no-win play, the physical space of the user can be recontextualized as a series of points or locations where shopping, typically considered a leisurely activity, becomes a turf war between players who seek to acquire the kicks before they run out. Even the inside of the store becomes an arena where shoppers no longer look for goods simply out of personal need, but rather utilize the store to repetitively find and scan objects for virtual currency. This hints that commercial space is no longer something that shoppers "enter" or "leave." Rather, commercial spaces are always with the shopper; the shopper's everyday activities, even those movements not associated with shopping directly, are monitored.

\section{Hyper-domicile: reframing hybrid space through playful mobile interfaces}

Analyzing pervasive games, de Souza e Silva (2008: 422-423) states that they include "features from communities in physical places and from cybercommunities, creating a type of communication that bridges differentiated spaces." 
Mobile devices, in concert with infrastructure, data, and social/spatial practice form hybrid spaces supported by social-mobile interfaces (de Souza e Silva 2006). In the context of hybrid spaces, interfaces are the "nexus" connecting human experience with object/medium/content. Simply put, an interface is a set of co-constituted, habituated relations produced through the body's interactions with outside systems and objects, rather than the interface as something being "imposed" upon the user (Farman 2012). Location-aware media and mobile devices can be construed as interfaces, or filters, which co-constitute technology, social interaction, location, and everyday spatial practice. Furthermore, interfaces also embody issues of control and privacy-mobile interfaces allow users increased control over their location and physical surroundings, but users also give up some traditional notions of privacy by submitting to both top-down and lateral surveillance at the hands of the program (de Souza e Silva and Frith 2012). This definition combined with the gamified mobile applications described above suggests that applications like McDonald's Monopoly and Shopkick represent a type of gamified mobile interface: They utilize mobile technology, location-awareness, and social practice to facilitate playful hybrid spaces.

Before I go any further, let me unpack what I mean by interface, and link it to the idea of a playful hybrid space. On the simplest level, an interface is a membrane between two systems that allows them to work in concert with each other. Poster (1996: 20) states: "Provisionally we may say that the interface stands between the human and the machinic, a kind of membrane dividing yet connecting two worlds that are alien to and also dependent upon each other." An interface can serve as a mediation device or translator between machines, material bodies, and perceptions. If we take the examples of location-based gamification like McDonald's Monopoly, then we can assume that gamified mobile interfaces facilitate the process of reframing spatially situated consumer practices.

As we saw in the examples of Shopkick and Monopoly, gamified environments do not necessarily "speak for themselves." The framing of space is reliant on the game mechanics working in tandem with other points of intersection such as design, environment, and intent. For instance, one could argue that locationbased applications like Shopkick are partial to dense urban areas where shops can be easily reached (and where more businesses are inclined to participate). Thus, the physical environment plays a key role in the mechanics of the application. Gane and Beer (2008: 55) state that we can "think in terms of interfaces within interfaces: of sets of interfaces embedded within or working in conjunction with one another." Thus, when designing gamified digital interfaces that mediate experience, designers must navigate certain productive tensions that are unique to the creation of hybrid spaces: The interfaces must be functional; they must operate through material and codified infrastructure; and they must embody aesthetic qualities that converge with the desires of users while working toward the end goals of the designer (Silver 2009). At the same time, interfaces are not simply immobile sets that translate information. The interface shapes perceptions 
of material and immaterial objects while also succumbing to cultural and computational logics of the things used to create them.

\section{Gamifed mobile interfaces and hybrid space}

The interface as a recursive filter is a useful context to keep in mind when considering how mobile interfaces such as Shopkick interact with and constitute hybrid spaces. Mitchell (2003) describes mobile, digital interfaces as an extension of perceptual and sensory systems that are integrated into the physical and virtual structures of city spaces and their architectural frames. Similarly, pervasive mobile interfaces are implicit in framing navigation by feeding information into lived environments (de Souza e Silva 2006). The interaction between digital mobile interfaces and physical environment brings hybrid spaces into being through synthesis. This image of hybrid space is an alternative vision to an interface being either a harbinger of the virtual or a membrane connecting two disparate modes of existence; de Souza e Silva (2006: 273) encourages a reframing of what "physical" and "digital" spaces actually entail. For example, users of the gamified mobile eating interface Belly (Rao 2012a) employ it as a tool for constructing and navigating a sensory eating experience in the urban environment. With the aid of Belly, users reframe their internal map of the city to reflect the data provided by the application, the physical realities of navigation and the game mechanics that drive the application. Mobile interfaces are designed filters that both control and are controlled by users (de Souza e Silva and Frith 2012). Thus, gamified mobile applications constitute frames through which our experiences of spaces are distributed - the world is ordered and reordered according to the desire of the designing entity and the desire of the user.

As we saw in the analysis of Monopoly and Shopkick, these games exert agency in the productive tension that is evoked by hybrid spaces. Gamified mobile interfaces do not just affect the user's circulation through and perception of physical spaces; the protocol of game mechanics also exerts a pull on the design and agency of mobile interfaces. For example, Shopkick's design largely reflects the simple rewards programs, like frequent flier miles, instituted in a predigital era. However, the real time, constant tracking of locational information allows for locational point-based elements to be included in the design, thus adding a sophisticated motivational layer nested within the more traditional rewards system. This also represents the two-way street mobile gamification occupies: Motivating consumers to engage generates useful marketing data for the corporations using the app. Thus, gamification in the context of mobile interfaces helps to politicize, frame, and territorialize experience. At the same time, gamified mobile applications extrapolate capital from areas of everyday activity that were not previously accessible to the surveillance tools of marketers. As playful applications and locational data mining collide, gamification in a mobile context is an emerging aspect of mobile marketing. Congruent to the deployment of gamified hybrid spaces, activities that previously were difficult or impossible 
to track in real time, such as shopping excursions or the consumption of extralarge soft drinks, become connected to larger networks of capital through surveillance mechanisms.

\section{Mobile domicile: the gamified house}

The mobile gamification of everyday life is not limited to commercial spaces. Domestic spaces are also being enmeshed into the net of gamified hybridity in the form of smart meters placed in homes and the platform Green Button, which allows application developers to create social applications using real-time data (Pegoraro 2012). So-called smart meters are implanted with wireless transponders that monitor and transmit the power consumption of a house in real time to a database. One goal of the meters was to reduce energy use and promote efficiency. However, being able to see power readings in nearly real time does not necessarily change behavior. Alongside these meters, third-party developers envisioned a system of competition where neighbors, neighborhoods, and even towns could compete against each other in real time, using cell phones to constantly check their ranking. Not only can users use cell phones to check their home's power usage, in many applications they can use a mobile interface to remote control lights, temperature, and other aspects of energy use while on the go.

This set-up is a very good example of a traditional reading of hybrid space. The mobile interface changes the context of the home and creates a social gaming experience that is both remote and contiguously context-aware. Also, domestic space exists not only as the hearth or home, a place for rest and leisure, it also becomes framed as a space that is simultaneously monitored and controlled. Scholars have noted that corporations and their associated technological apparatuses have been implicit in the colonization of domestic space and, by extension, the lifeworld since the techno-managerial revolution that occurred during the 1980s (Deetz 1992). In fact, gamification participates in a process that Deetz (1992) terms naturalization, or pervasive corporate management systems' ability to introduce discursive formations that suppose the management of subjects and spaces is absolutely necessary for efficiency, rather than invasive. What this may represent is a folding of the market and the state into spaces that previously were not accessible. With the introduction of networked home technologies, domestic spaces provide new markets that can be enfolded into the digital enclosure. Like the city grid in Monopoly and the locations of bubbles in Shopkick, the home is reframed in the context of game mechanics embedded in a mobile interface.

Smart meters make a game out of controlling the internal climate while also generating the possibility of checking and controlling the internal built environment while moving through the city. One is still "home" while not physically inhabiting the architectural structure of the house. Also, the smart meters and corresponding gamified system reframes domestic space and gamespace where 
real-time competition with other homeowners occurs both locally and nationally. The placing of smart meters is contiguous with the physical realities of the house, however with the addition of a gamified system, the perception of the physical realities is altered. This is similar to Virilio's concept of "domotics" (Redhead 2004), although technically a direct inverse. Virilio assumed that technology would place the house in constant motion (door and windows automatically adjusting to the dweller) while the person living in the house would be completely stationary. Furthermore, screens mediate the dweller's perceptions of the house and the outside world (Redhead 2004). Yet mobile interfaces like Green Button seem to facilitate the house moving with a mobile dweller. In this case, the "screen" involved is far more complex than the sedentarist "plugged in" concept: Rather than the motionless virtuality of Virilio's domotics, we are moving toward the hyper-mobile (hybrid) domicile facilitated, in part, through a mobile interface. This form of playful domotics is connected to the creation of different subjects, where homeowners and shoppers become active participants in a consumptive cycle that utilizes playful behaviors that operate in concert with behavioral and surveillance protocols.

\section{Discussion}

Gamified mobile interfaces can represent many differing applications, both commercial and non-commercial. Some critics argue that non-commercial applications are "playified" (Nicholson 2012), a term I disagree with because it assumes that play can somehow be free of commercial or disciplinary influences. If one takes even a quick look at the booming game industry it is clear that play, capital, and commercialism are not at odds (Dyer-Witheford and de Peuter 2009). Implicit in these claims is that gamified colonization of spaces does not necessarily destroy environments, but reimagines them as spaces that are naturally prone to management and commoditization in the first place. Intents aside, gamification in any form presents some ethical considerations. All of the applications noted above are arranged around the user's access to certain spaces and technologies. We must take into account that these applications, by design, have certain pleasures in mind for users. Conversely, they intentionally obscure other pleasures embedded around them. For instance, the promise of extrinsic rewards in Monopoly may also cause users to bypass locally owned restaurants or drive further in order to collect game pieces.

As commercial applications such as Shopkick, Monopoly, Belly, and Waze become more ingrained in urban users' everyday lives, we must pay special attention to the interactions between designed mobile interfaces and spatial politics. Gamification is not necessarily insidious, but it does present new issues when it comes to mobile practice and leisure. For example, a person in public housing would probably not have the option of playing the applications enabled by Green Button. In addition, applications like Shopkick work best in concentrated urban environments rather than rural areas because there are more 
"bubbles" located in a smaller area. Such concerns highlight possible tensions between privileged urban areas and rural or poor communities. These tensions are indicative sets of power-geometries (Massey 1993) in which social groups have uneven access to the virtual currencies of location-based applications. Thus it is not just a question of who can successfully use these applications for pleasure, it is also about who is turning a profit by creating them.

Harvey (2006: 101) notes that "spatially fixed and immobile infrastructures of transport and communications systems ... are required in order to liberate other forms of capital and labor for easy spatial movement." The deployment of communication technologies and their associated practices (such as gamification) contribute to uneven geographic development, or the unequal distribution of materials in space as they are related to global circulations of capital (Harvey 2006). In this context, using virtual currencies to commodify mobile practice hinges on what types of customers marketers are trying to reach. Inevitably those who accrue virtual currencies are doing so through access to the systems themselves. The spaces in which gamification is deployed reflect the socio-economic and spatial situation of the "ideal" user, namely the users most useful to the system. This could be expanded to include the notion that the lifeworld (Husserl 1970; Habermas 1987), or social spaces in which corporate and governmental interests do not traditionally intervene, are being actively colonized by technologies that assume from their inception the idea that no spaces, not even playful ones, should or can exist without direct interference and monitoring.

\section{Conclusion}

I have given a brief illustration of various platforms through which gamification occurs and proposed that gamification is a technique utilizing game mechanics embedded in larger systems. Gamification, in context, seeks to create more engagement with the locations, objects, and systems that it modifies. In combination with mobile technologies and data mining, applications like Shopkick represent an opportunity for increasing the reactive and predictive capabilities of systems through surveillance. When implemented through location-aware mobile devices, I argue that gamified applications contribute to the creation of a unique, playful form of hybrid space that reframes spaces and everyday activities. Monopoly and Green Button both illustrate how gamified mobile interfaces utilize game mechanics to alter behavior and modify the context or frame of physical surroundings and mobile practices. Gamification, as a set of corporate, technological, and mobile practices, contributes directly to understanding how hybrid spaces are not just involved in the perpetuation of new forms of spatiality or sociability. They are also indicative of how protocol, data, and surveillance become integrated into everyday activities such as domestic life and personal shopping experiences.

Gamification works as a technique that informs the design of applications with control and efficiency in mind. It affects experience, movement, and spatial 
arrangements. At the moment, gamification is a concept that is surrounded by hype; many critics speculate it is heading to the "trough of disillusionment" (Goad 2011). However, the success of applications like Shopkick and Monopoly suggests that some gamified applications are capable of generating large amounts of cash and valuable information. While this chapter lays some groundwork for gamification in a mobilities context - specifically the relationships between gamification and everyday mobilities - there are several tasks that need to be done. Exploring the cultures of design and production in relation to gamification can possibly give a much better insight as to the direction of the trend. Interviewing designers also grants insight into the intentions and concerns of those with the "upper hand." Also, many of these applications have a presence on social media sites, which can offer a wealth of public feedback and may provide quantitative data for empirical analysis. Both the design culture behind gamification and the experiences of users are ripe for exploration. The gamification hype makes tracking the effective/affective nature of applications an important task for mobilities researchers. Gamification divides and dissolves the lines between play, consumerism, work, and leisure. It reframes space and changes perceptions. Proper examination will give a more robust portrait of how our cities of the instant are shaped by play.

\section{References}

Andrejevic, M. (2006) The discipline of watching: Detection, risk, and lateral surveillance, Critical Studies in Media Communication, 23(5): 391-407.

Andrejevic, M. (2007) Surveillance in the digital enclosure, The Communication Review, 10(4): 295-317.

ASTD Staff (2012) Broadening the definition of gamification for L\&D professionals, Learning Circuits, available at: www.astd.org/Publications/Blogs/LearningTechnologies-Blog/2012/01/Broadening-the-Definition-of-Gamification-for-LDProfessionals.

Austin, D. (2014) The gamification of energy conservation, DZone Social Business, January 7, available at: www.dzone.com/articles/gamification-energy.

Baudrillard, J. (1998) The drama of leisure or the impossibility of wasting one's time, in J. Baudrillard, The Consumer Society: Myths and Structures, Thousand Oaks, CA: Sage, pp. 151-158.

Benford, S. and G. Giannachi (2011) Hybrid space: Between real and virtual, local and global, in S. Benford and G. Giannachi, Performing Mixed Reality, Cambridge, MA: MIT Press, pp. 27-70.

Bogost, I. (2007) Procedural rhetoric, in I. Bogost, Persuasive Games: The Expressive Power of Video Games, Kindle edition, Cambridge, MA: MIT Press.

Campbell, M. (2011) The audacious plan to make the world into a game, New Scientist, 209(2794): 36-39.

Chan, D. (2008) Convergence, connectivity, and the case of Japanese mobile gaming, Games and Culture, 3(1): 13-25.

Chief Marketer (2010) Monopoly at McDonald's: 2011 IMA Award Winner, Chief Marketer, available at: www.chiefmarketer.com/mobile-marketing/2010-monopoly-atmcdonalds-2011-ima-award-winner-31122011. 
Cronin, A. (2006) Advertising and the metabolism of the city: Urban space, commodity rhythm, Environment and Planning D: Society and Space, 24(4): 615-632.

Crunchbase (2011) Shopkick Company Profile, available at: www.crunchbase.com/ company/shopkick.

de Souza e Silva, A. (2006) From cyber to hybrid: Mobile technologies as interfaces of hybrid spaces, Space and Culture, 9(3): 261-278.

de Souza e Silva, A. (2008) Hybrid reality and location-based gaming: Redefining mobility and game spaces in urban environments, Simulation \& Gaming, 40(3): 404-424.

de Souza e Silva, A. and J. Frith (2012) Mobile Interfaces in Public Spaces: Locational Privacy, Control, and Urban Sociability, New York, NY: Routledge.

de Souza e Silva, A. and L. Hjorth (2009) Playful urban spaces: A historical approach to mobile games, Simulation \& Gaming, 40(5): 602-625.

Deetz, S. A. (1992) Corporate colonization of the lifeworld, in S. A. Deetz, Democracy in and Age of Corporate Colonization: Developments in Communication and the Politics of Everyday Life, Albany, NY: State University of New York Press, pp. 13-44.

Delo, C. (2012) What is gamification, and how can I make it useful for my brand? Advertising Age, 83(9): 58.

Dieckmann, P., S. M. Friis, A. Lippert, and D. Østergaard (2012) Goals, success factors, and barriers for simulation-based learning: A qualitative interview study in health care. Simulation \& Gaming, 43(5): 627-647.

Dyer-Witheford, N. and G. de Peuter (2009) Games of Empire: Capitalism and Video Games, Minneapolis, MN: University of Minnesota Press.

Ehrenberg, R. (2010) Molecules: In world of proteincraft, humans win: People solve molecular puzzles in online computer game, Science News, 178(5): 7.

Farman, J. (2012) Mobile Interface Theory: Embodied Space and Locative Media, New York, NY: Routledge.

Flintham, M., K. Smith, S. Benford, M. Capra, J. Green, C. Greenhalgh, M. Wright, M. Adams, N. Tandavanitj, J. R. Farr, and I. Lindt (2007) Day of the figurines: A slow narrative-driven game for mobile phones using text messaging, in M. Flintham (ed.), 4th International Symposium on Pervasive Gaming Applications (Pergames 2007), Salzburg, Austria, Aachen: Shaker Verlag.

Gane, N. and Beer, D. (2008) New Media: The Key Concepts, New York, NY: Berg.

Gazzard, A. (2011) Location, location, location: Collecting space and place in mobile media, Convergence: The International Journal of Research into New Media Technologies, 17(4): 405-417.

Goad, L. (2011) Gartner: Gamification's hype is about to reach its peak, ZDNet, available at: www.zdnet.com/blog/gamification/gartner-gamifications-hype-is-about-to-reach-itspeak/583.

Habermas, J. (1987) The Theory of Communicative Action, Volume 2: Lifeworld and System: A Critique of Functionalist Reason, Boston, MA: Beacon Press.

Harley, J. B. (2001) Maps, knowledge and power, in P. Laxton (ed.), The New Nature of Maps: Essays in the History of Cartography, Baltimore, MD: Johns Hopkins University Press, pp. 51-82.

Harvey, D. (2006) Notes towards a theory of uneven geographical development, in D. Harvey, Spaces of Global Capitalism: Towards a Theory of Uneven Geographical Development. New York, NY: Verso, pp. 69-116.

Husserl, E. (1970) The Crisis of European Sciences and Transcendental Phenomenology: 
An Introduction to Phenomenological Philosophy, Evanston, IL: Northwestern University Press.

Jacobs, P. (2012) Emergence of human techsourcing. Human Resources Magazine, 16(6): $14-15$.

Kriz, W. C. (2009) Bridging the gap: Transforming knowledge into action through gaming and simulation, Simulation \& Gaming, 40(1): 28-29.

Lawson, L. L. and C. L. Lawson (2010) Video game-based methodology for business research, Simulation \& Gaming, 41(3): 360-373.

Licoppe, C. (2009) Recognizing mutual "proximity" at a distance: Weaving together mobility, sociality and technology, Journal of Pragmatics, 41(10): 1924-1937.

Licoppe, C. and Y. Inada (2008) Geolocalized technologies, location-aware communities, and personal territories: The Mogi case, Journal of Urban Technology, 15(3): 5-24.

Licoppe, C. and Y. Inada (2010) Locative media and cultures of mediated proximity: The case of the Mogi game location-aware community, Environment and Planning D: Society and Space, 28(4): 691-709.

Liyakasa, K. (2012a) Serious about gamification, CRM Magazine, 16(8): 33.

Liyakasa, K. (2012b) Turning business into pleasure, CRM Magazine, 16(3): 14.

Loftus, G. and E. Loftus (1983) Mind at Play: The Psychology of Video Games, New York, NY: Basic Books.

Lopez, J. (2012) Three ways to overcome the commuter blues with gamification, Gamification Corp, available at: www.gamification.co/2012/01/11/three-ways-to-overcomethe-commuter-blues-with-gamification/.

Massey, D. (1993) Power-geometry and a progressive sense of place, in J. Bird, B. Curtis, T. Putnam, and L. Tickner (eds.), Mapping the Futures: Local Cultures, Global Change, London: Open University Press, pp. 60-70.

Massey, N. (2012) Can "gamification" make your life more sustainable? E\&E News, ClimateWire, February 3, available at: www.eenews.net/climatewire/stories/ 1059959392.

Mitchell, W. J. (2003) Me++: The Cyborg Self and the Networked City, Cambridge, MA: MIT Press.

Montola, M., J. Stenros, and A. Waern (2009) Pervasive Games: Theory and Design, Burlington, MA: Morgan Kaufman Publishers.

Murff, E. (2011) Changing the world through meaningful play: Report on the 2010 ISAGA Conference, Simulation \& Gaming, 42(1): 127-130.

Nicholson, S. (2012) A user-centered theoretical framework for meaningful gamification, paper presented at Games + Learning + Society 8.0, Madison, WI.

Pegoraro, R. (2012) Gamification: Green tech makes energy use a game - and we all win, Ars Technica, available at: http://arstechnica.com/features/2012/02/ gamificationgreen-tech-makes-energy-use-a-gameand-we-all-win/.

Poster, M. (1996) The Second Media Age, Cambridge: Polity Press.

Rao, L. (2012a) Customer loyalty and rewards platform for local businesses Belly raises $\$ 10 \mathrm{~m}$ from Andreessen Horowitz, TechCrunch, available at: http://techcrunch. com/2012/05/08/customer-loyalty-and-rewards-platform-for-local-businesses-bellyraises-10m-from-andreessen-horowitz/.

Rao, L. (2012b) Location-based shopping app Shopkick now 3 million users strong; 1B deals viewed, TechCrunch, available at: http://techcrunch.com/2012/01/24/ locationbased-shopping-app-shopkick-now-3-million-users-strong/.

Redhead, S. (2004) Accelerated culture, in S. Redhead, Paul Virilio: Theorist for an Accelerated Culture, Edinburgh: University of Edinburgh Press, pp. 37-70. 
Savitz, E. and K. Heisler (2012) Let's play: To keep Gen Y staffers, gamify their work, Forbes.Com, 41, available at: www.forbes.com/sites/ciocentral/2012/07/03/lets-playto-keep-gen-y-staffers-gamify-their-work/.

Scofidio, B. (2012) Get serious about gamification, Corporate Meetings \& Incentives, 31(7): 2.

Sennott, S. (2005) Gaming the ad, Newsweek, 145(5): E2.

Sicart, M. (2008) Defining game mechanics, Game Studies, 8(2), available at: http:// gamestudies.org/0802/articles/sicart.

Silver, M. (2009) Exploring Interface Design, New York, NY: Cengage.

Stenros, J., A. Waern, and M. Montola (2012) Studying the elusive experience in pervasive games, Simulation \& Gaming, 43(3): 339-355.

Sutko, D. M. and A. de Souza e Silva (2008) Playing life and living play: How hybrid reality games reframe space, play, and the ordinary, Critical Studies in Media Communication, 25: 447-465.

Swan, C. (2012) Gamification: A new way to shape behavior, Communication World, 29(3): 13-14.

The Economist (2011) All the world's a game, December 10, The Economist, available at: www.economist.com/node/21541164.

Urry, J. (1995) Time and space in the consumption of place, in J. Urry, Consuming Places, New York, NY: Routledge, pp. 1-33.

Wasserman, T. (2013) Google buys Waze, June 15, available at: http://mashable. com/2013/06/11/google-buys-waze/.

Wilken, R. and J. Sinclair (2009) "Waiting for the kiss of life": Mobile media and advertising, Convergence: The International Journal of Research into New Media Technologies, 15(4): 427-445.

Wilson, M. W. (2012) Location-based services, conspicuous mobility, and the locationaware future, Geoforum, 43(6): 1266-1275.

Zicherman, G. and C. Cunningham (2011) Gamification by Design: Implementing Game Mechanics in Web and Mobile Apps, New York, NY: O'Reilly Media.

Zicherman, G. and J. Linder (2010) Game-Based Marketing: Inspire Customer Loyalty Through Rewards, Challenges and Contests, Hoboken, NJ: Wiley.

Zuk, R. (2012) Get in the game: How communicators can leverage gamification, Public Relations Tactics, 19(2): 7. 
Mobile cities

Mapping, architecture, and

planning 
This page intentionally left blank 


\title{
Chapter 10
}

\section{Exploring locative media for cultural mapping}

\author{
Peter Hemmersam, Jonny Aspen, \\ Andrew Morrison, Idunn Sem, and \\ Martin Havnør
}

Today's cities are teeming with mobile communication. Our interest in the design and uptake of mobile media is to do with its potential to both read and write the city by annotating and informing the mappings and readings of urban space. A growing body of research is emerging on how mobile technologies are taken up by different interest groups, such as the Occupy Movement (CostanzaChock 2012; New York Times 2012) and public locatively mediated protest; in relation to personal identity, such as teens' uses of cell phones (Ito 2009); and primarily in relation to information, navigation, and the push of services in the context of the mediated city (Hjorth et al. 2012). This research is racing to keep up with the burgeoning spread of mobile applications or "apps" in daily life in urban settings. Increasingly, it is becoming apparent that we live and work in what we call the "networked city" (Campbell 2012; Graham and Marvin 2001; Rainie and Wellman 2012) that is a blend of the physical and digital. Our built urban settings are now infused with social media and information communication technologies that are manifested, culturally and communicatively, through our own productive engagement - such as the use of digital urban screens (Pop et al. 2012) and the use of media players and smartphones in urban space (Bull 2005; de Souza e Silva and Frith 2012). Mobile apps such as Foursquare allow users to check in to a location and to broadcast their location to friends; mixed and augmented reality tools such as Layar facilitate the meta-annotation of urban spaces. We are now also witnessing the rapid spread of locative social media such as these being connected to Facebook and Google in wider discursive networks.

These developments have occurred alongside an emerging and now solid body of research into locative media and place-specific computing (Gordon and de Souza e Silva 2011; Lemos 2010; Messeter 2009), including ubiquitous aspects (Dourish and Bell 2011) and occasionally planning (Gordon and de Souza e Silva 2011). Yet there is still scant research that critically explores relations of locative and social media to the mapping and design of cities (e.g., Speed and Southern 2010; Shepard 2011, Morrison et al. 2013). This is our primary interest in this chapter. We characterize this as cultural mapping akin to that already covered in cultural geography (Roberts 2012). In linking mobility, 
mobile communication, and locative media, we situate cultural mapping within an urbanism of the networked city. This is why the concept is used somewhat differently to the more mainstream one applied in cultural geographic resource mapping and cartography.

In our usage, cultural mapping on the one hand covers mapping practices that are essentially creative and experimental readings of urban space, but also relating to architecture and the design of cities. On the other hand, it encapsulates mapping "features" as culturally co-constructed and integral to actual mapping performance in the field. The term therefore refers to more participative and dynamic representational roles that mobile media open up, at the same time as it captures prevalent practices and attitudes of urban living. This concerns our persistent involvement in everyday urban matters through the performance of both active interpretation and subjective intervention that may now also be extended to locative and social media tools and emergent practices of "reading and writing" how we perceive, shape, and experience the city via social media and related place-based communication. These communicative activities are enabled by locative media applications and the affordances of smartphones. However, as we discuss below, they are also realized through our actions and how we go about participative cultural mapping-similar to grassroots or bottom-up approaches (Crawford 2008) — in contrast to being recipients of top-down urban planning.

In the design, teaching, and research we report on below we connect the quickly expanding domain of mobile media to an experimental research inquiry into locative media. This inquiry transposes a previous established and well-tried paper-based and face-to-face method for cultural urban mapping into an application for locative, mobile mapping (Morrison et al. 2012a). This inquiry takes place as part of a large research project called YOUrban that looks into social media, the city, and performativity. As part of the project a collaborative and interdisciplinary team-including researchers from urbanism and architecture, communication design and informatics, media and interaction designers, as well as a mobile software company_-developed a collaborative urban mapping platform in the form of an iPhone application called Streetscape. We built Streetscape to explore the potential of using locative mobile media as a tool for developing experimental forms of collaborative cultural mapping in urban settings.

Streetscape goes beyond traditional cultural resource mapping in that it allows users to identify and apply experimental, locative, and mobile mapping perspectives established in urbanism so as to investigate ways of looking at and engaging in active agencies of selection and annotation. In this way Streetscape is a prompt for thinking about how cities are designed and choreographed (Corner 1999) and curated (Bunschoten 2001). As part of its experimental design and development, the app was used and evaluated in a large master's-level class in urbanism that enabled active productive participation by groups of students of architecture. In the following sections we offer reflections on the design and use 
of the first version of Streetscape, primarily based on evaluation material from testing the app in a workshop with sixty students in August 2011. One of the key questions we investigated was how acts of urban mapping might be influenced when transposed to a mobile app. We were also motivated to use Streetscape to heuristically examine the potential for the further development of apps for cultural mapping in urban contexts.

As part of a wider investigation into social media, the city, and performativity called YOUrban, we co-designed and developed Streetscape as a locative mobile application. Behind this app lay experience in using a paper-based experimental mapping methodology from Urban Gallery (developed by Raoul Bunschoten and CHORA 2001). In Streetscape we transposed this tool into a GPS-based mapping tool for the iPhone (Morrison et al. 2012a; Morrison and Aspen 2013). Our aim was to see how the four perspectives of Urban Gallery-Erasure, Transformation, Origination, and Migration - might be used to develop what we have called cultural mapping of the networked city. This mapping is based on Points of Interest or POIs, a convention now widespread in mobile maps, to locate image, text, and tags in the context of a mapped city. Actual mappings of the city are carried out onsite and through movement; and the author and others may access these mappings similarly. It is the exploratory reading and "writing" of the city that marks these activities as cultural mappings onsite and on the move - that is, informationally, interactionally, and interpretatively.

The chapter proceeds as follows: We start out by presenting urban mapping as a context for our research and design and we locate the app in a locative media context. Thereafter we give a short description of the Urban Gallery methodology on which we drew in designing Streetscape. In the second part of the chapter we look more closely into the design and functionality of the actual app and then present reflections upon its design and use. We include screengrabs of the app and photos of contexts of use. We end by presenting what we see as key issues in a discussion of potential for further development of locative media as tools for creative and experimental urban mapping. We suggest that through locative and mobile media our understanding of our urban spaces and lives may be more fully understood as environments that can be culturally mapped, dialogically and collaboratively. This may be conducted in relation to place specifics and to the emergence of unscripted features and occurrences that we identify, negotiate, and share, differing considerably in tone from our experiences as city dwellers embedded in the predominant and often seemingly distant discourses of urban planning.

\section{Urban mapping}

Mapping technologies such as Geographical Information Systems (GIS) have been applied in planning and research in urbanism since the 1960s. The coupling of databases and geographic location has proved to be a powerful tool in planning and modeling urban development. In addition to GIS, satellite and aerial 
imaging and other forms of remote sensing made available through new mobile and locative technologies have produced forms of representation that can be described as "viewing the earth from without"; this can be understood as "a dominating trend in current understandings of geography and space" (Anker and Anker 2011: 89), which is also found within fields of urban and landscape mapping. Such modes of mapping can be considered as contemporary descendants of mainstream modernist mapping approaches based on statistical methods. They have proven effective in combining environmental, infrastructural, and statistical data in analysis of cities and landscapes. They have also contributed to the mobilization of "narrow managerial rationalities at the expense of a more widely defined human condition" (Anker and Anker 2011: 89). Critiques of modernist mapping practices notably include Critical Cartography (Harley 1989; Wood and Fels 1992) in which the political reading of the power behind maps is promoted (such as exposing colonial or sexist logics). Denis Cosgrove has also demonstrated how mapping by fixing borders and demarcating territory is thereby setting legal and economic frameworks, and that the sorting and ordering implicit in mapping are "regarded as defining features of a 'modern' mentality" (Cosgrove 1999: 4).

Running parallel to such modes of mapping, we find more postmodern approaches to mapping cities and urban features. This alternative tradition of mapping arguably began in the late 1950s with the growing critique of the statistical generalizations that had informed modernism in planning and proposed techniques that focused on individual perspectives, "viewing the world from within" (Anker and Anker 2011), and on issues of identity rather than rationalization and generalizations. Prominent early advocates of such an approach include Kevin Lynch (1960) and Jane Jacobs (1961), as well as the more experimental urban mapping approaches of the Situationist International. A key aspect in postmodern mapping was to integrate individual perspectives locating the observer in lived and experienced spaces, as well as attempts at mapping relations between people and their environment that extend beyond the merely utilitarian. One can also see an increasing interest in exploring more subjective ways of reading the city in terms of lifestyles, local specifics, cultural experiences, and conditions. According to Jacobs (1961: 229), planners should "aim for areas of great diversity and, because real differences are thereby expressed, can get results which, at worst, are merely interesting, and at best can be delightful." Identification of individual desires and sensitivity to particular group interests, as well as to issues of identity, difference, and differentiation, has since Jacobs' time evolved into a major interest among many urban planners, urban designers, and urban theorists.

Lynch and Jacobs pursued perspectives that were local, place-specific, and related to individual perception of the city; both of them aimed at developing models or certain "fixtures" for the development and design of cities. Lynch (1960) proposed a set of five mental elements of urban space: paths, districts, edges, nodes, and landmarks. Jacobs (1961) described four indispensable 
conditions for the generation of "exuberant diversity in a city's streets and districts": mixed-use, short blocks, buildings of varying age and condition, and a dense concentration of people. While both Lynch and Jacobs pursued a "complex and highly developed form of order" (Jacobs 1961: 222), it was an order that was hidden from immediate perception, requiring new techniques in order to be explored, captured, and given representational form. The techniques of postmodern mapping have subsequently emphasized the accidental and serendipitous in mapping as an important approach to identify hidden or unseen potentials of urban living and for urban development that could pose as alternatives to the logics of mainstream urban planning, such as "Strategic Regional Planning" (Albrechts et al. 2003), "Transport Oriented Development" (Calthorpe 1993) but also participatory planning based on notions of communicative planning (Forester 1989; Healey 1992) which is often found wanting with regards to expected outcome (Innes and Booher 2004; Forester 1989).

Difficulties with application of techniques of collectively mapping individual perspectives often left actual translation of these methods to professional or "trained observer[s]" (Lynch 1960: 143). Here we see a productive role for locative social media in highlighting individual and alternative voices - a new affordance of the networked city.

However, postmodern mapping and the "paradigm of identity" (Nielsen 2008) in planning with which it is often associated has been criticized as conservative and out of touch with the radical forces that drive urban development, for celebrating a focus on the individual in urban development and for contributing to a conservative approach or "NIMBY-ism" (Not In My BackYard). A critique of mapping based on the individual perspective and the local is that it can only lead to designing the local, and "[t]he problem is that ... small scale, bottom-up, and eco-friendly moves ... do not address fundamental issues of social justice and equity that are also part of the foundations of a true urbanity" (Shane 2004: 7). Even though much of the criticism might be correct, we would propose that an interest in the individual and local, and in issues of difference and heterogeneity in all matters urban-and in how such features may be traced, mapped, and represented - also in the future will be an important issue on the agenda of urbanism, especially as a means for combating more homogenizing forces of urban development as well as for imagining alternative urban futures.

For these reasons mapping still remains important in urbanism and the search for alternative approaches continues. In recent decades we have seen a significant shift away from a primarily architecturally conditioned understanding of space and place toward a more social and geographically informed discourse of space as "a social product — one less designed and constructed than enacted or performed through specific behaviors and practices" (Shepard 2011: 22; see also de Certeau 1984; Harvey 1996; Lefebvre 1991).

The renewed interest in mapping and urban representation can also be seen as an effort to get to grips with the changing nature of the contemporary city, and 
as a way of engaging with the dynamics and flows of globalizing cities. In James Corner's words, it is

becoming clearer to architects and planners that "space" is more complex and dynamic than previous formal models allowed. Ideas about spatiality are moving away from physical objects and forms towards the variety of territorial, political and psychological social processes that flow through space.

(Corner 1999: 227)

Thus the challenge is to explore ways of mapping aspects of space understood as "a field of connections, relationships, extensions and potentials" (Corner 1999: 224). Increasingly, experimental mapping in architecture, urbanism, and landscape studies aims at getting inside the complexities of space-mapping urban flows and invisibles (Allen 2000; Amoroso 2010). Emerging forms of mapping cover the interaction of human and non-human actors (Yaneva 2012), joining individual perspectives, and relating them to overarching topics and dynamics of urban planning and development.

\section{Mobile mapping}

Urban mapping forms an important background for the development of Streetscape, as do media perspectives on mapping and geolocation. Mobile annotation is the act of attaching information to locations, resulting from the development of wireless technologies with GPS as its most important element, and has led to widely used urban navigation systems.

[L]ocation-based services can also enable ordinary people to tie bits of media and information to specific locations in the physical world, marking up the built environment with personal notations, stories and images. Less common are applications that explore the ability of GPS enabled technologies to correlate place, time and identity in ways that empower users to not just read information, but also write it.

(Shepard 2011: 25)

Early investigations into such new forms of space appropriation were to a large extent initiated via media art projects such as the groundbreaking Urban Tapestries (started in 2002) which located stories in urban space through "public authoring" and used handheld devices for authoring and retrieving information when moving through space (www.urbantapestries.net). Urban Tapestries was tested with actual partners and was developed into a platform that specifically aimed at "staging creative interventions with local areas and communities that engage with regeneration and environmental issues to increase issue ownership and people's sense of agency and empowerment to act" (Proboscis 2007). 
Media art projects, including those of Blast Theory and academic investigations of urban annotation approaches such as Urban Tapestries can be seen as attempts to "challenge accepted interpretations and uses of space" (Gordon and de Souza e Silva 2011: 52). With the widespread ownership of GPS-capable mobile devices in the form of smartphones, these have been followed by an array of commercial services, many of which have been about navigation and services (for example the gay-oriented meeting service Grindr and the imaging tool Instagram). In contrast to commercial payment subscription apps, experimental and critical approach of projects like Urban Tapestries inscribe public authoring and open and free access. Such a tool provides a "reminder that people are not just consumers" and what is on offer is "a model of playful experimentation and knowledge aggregation for society to question and understand how we can be social beings in a networked world" (Proboscis 2006).

While the representation of the individual perspective is clearly important in Urban Tapestries, "the project ... focused less on the uniqueness of individual expressions and more on the aggregation of these personal annotations and how they form a collective representation of urban life in a particular place at a particular time" (Shepard 2011: 28). As a media project it represents an attempt to take a "step beyond [the then] current uses of pervasive mobile systems" into a collective, bottom-up authoring of urban space (Proboscis 2004).

Collaboration and participant-based mapping have become important features in the changing relations between digital tools and technologies and how we constitute and communicate mappings of the urban (Gordon et al. 2011; Gordon and Manosevitch 2011). New mapping tools are influenced by open source software and practices as well as techniques of crowd sourcing. This can be seen in the OpenStreetMap initiative (www.openstreetmap.org) and the participative mapping platform Ushahidi (www.ushahidi.com) that has enabled citizen-based mapping of political violence in urban Kenya and the marshaling of global resource responses to the Haiti earthquake of 2010. Together with such emergent and distributive approaches, and through notions of crowd sourcing and collaborative authoring, local perspectives and serendipitous activities - rather than the simply random - are becoming recognized as critical modes of knowledge production and mapping approaches that can help shift the frames for reading, designing, and inhabiting the city.

\section{Urban Gallery}

As a well-tried precursor to such an approach, the Urban Gallery methodology developed by Raoul Bunschoten (2001) and the architectural research practice CHORA, which started in 1993, provides a tool and an activity-centered way to explore dynamic principles of mapping complexities of urban space and place. This methodology is an example of the tactical appropriation of place through creative modes of annotation and indexing that emerge through the application of a set of parameters to physical and spatial contexts. Urban Gallery was devised to deal with complex urban and regional situations, often marked by 
conflict and change, such as post-communist Bucharest (Bunschoten 1997) or the conflict zone comprising both sides of the Taiwan Strait (CHORA 2010). In the Bucharest project "the fluvial system of the Black Sea [is used] as metaphor, dynamic model and cultural icon, and considers the processes of change and how these might be harnessed as instruments that create operations of controlled urban change" (Bunschoten 1997: 82); and in the Taiwan Strait project the global phenomena of climate change is observed in local situations, resulting in proposals for sixty-five "prototypes" or interventions of an architectural and/or organizational character involving sets of actors from both sides of the strait. Raoul Bunschoten (2001: 27) explains the thinking behind Urban Gallery in this way:

emergent phenomena in urban environments are caused by proto-urban conditions. Proto-urban conditions belong to the part of the cities that are global, exceed local conditions. Observational methods can be developed to "see" proto-urban conditions in local samples. Tools can be made to describe the mechanics of dynamic processes in sample situations, relationships between these mechanics can be described in metaspaces, vehicles for models. The behaviour can be influenced, its organizational form altered, alternative developments imagined and communicated. Urban design can incorporate prototypes that use and enable emergent phenomena to construct new organizational forms, to mutate existing ones, to manage change. Architects are designers of spaces for emergent phenomena, for social, political, economic and cultural change.

The first phase of Urban Gallery focuses on mapping "proto-urban conditions" through observational methods, in order to build a database of "people, places and organisations that are somehow related to a project" enabling the proposal of "prototypes [which] are designs or organisational structures that address the issues raised in the database" (Awan et al. 2011: 118). The proto-urban conditions can be captured through four perspectives representing change:

1 Erasure (the act of erasing, obliterating, emptying out, and making space for something to happen). Examples of Erasure given to students included: the demolition of buildings; the blocking of sightlines;

2 Origination (the coming into existence of something new, change, or turning points emerging from within or added from the outside). Examples given to students included: new inhabitants moving in; an invention;

3 Transformation (a gradual or sudden change in character, form, appearance, or function - as a result of an internal dynamics or an external force). Examples given to students include: the recycling of garbage; the changing use of an urban space; and

4 Migration (an instance of mobility - things, people, or events moving about, a change in position). Examples given to students include: the movement of cars; the relocation of cultural events. 
Through random sampling (literally throwing handfuls of beans on a large paper map) urban situations are selected. They represent emergent micro perspectives on, for example, a special immigrant youth group gathering taking place in an obscure courtyard, that would otherwise go unseen or be unrecognized in traditional mapping techniques that center on pre-specified categories. The observed phenomena in Urban Gallery are described through attribution of images, names, actors, and agents. In the original Urban Gallery format participants are asked to fill out paper forms to note physical and iconographic features, such as a street sign made of steel and produced in Germany that is part of a wider infrastructural system of socio-cultural semiotic orientation, and involve the people behind its appearance, as well as the users. The mapping involved is "often highly personalized and peculiar to places and individuals. In this way, the fieldworker/mapper gains a remarkably detailed and socially colourful sense of local dynamics and desires" (Corner 1999: 243).

Large sets of observations make up databases that then form the basis for the later stages of the Urban Gallery, including the performance of scenario games, in which agents and iconographies are combined with the affordances of space and objects by the players in increasingly complex configurations. In these configurations material features and users are combined in new and unforeseen ways based on the initial observations and form operational prototypes. Scenario games physically gather local actors around maps and game-like interaction between players occur, in which the contents of the database is activated. Urban prototypes are organizational entities that connect with the dynamics of the city. The Guggenheim Museum in Bilbao became a prototype because of its iconic design that attracted tourists and was emulated by other cities around the world (Bunschoten 2010). These prototypes then form the basis of action plans, through which proposals (physical and organizational) are made or reinserted into planning or design processes as they are carried out by local or regional authorities, seeking to align their interests with local actors. Action plans may include architectural design proposals or financial and regulatory initiatives or proposals for new public and/or private organizations. Through these game-like structures and scenario building, local observations are connected across scales, from very local to global ones, transgressing the apparent restriction of the locality in which the observations were initially made. The action plans are formulated in the local context, but informed by regional and global as well as local resources and potentials through actual naming of flows of economies and resources, potential stakeholders and actors, as well as local physical conditions. Urban Gallery works by linking

the various cultural aspirations of each group to a physical space or territory, disguising amongst "local authorities" who anchor conditions into specific institutions or places, "actors" who participate with stated desires and "agents" who have the power and capacity to make things happen

(Corner 1999: 243) 
An example is the proposal to let a football club take part in the regeneration of an area in the Bucharest project (Bunschoten 1997).

According to Bunschoten the approach works "at the opposite end of the architectural spectrum: poetic form and material narratives, space as the vehicle for narrative meaning" (2007: 385). The frames set up through the methodology are not meant "to predetermine or preconfigure the outcome but rather to instigate, support and enable social forms of interaction, affiliation and negotiation" (Corner 1999: 243). The outcomes link people to space through narrative in line with the perspective of postmodern planning. Furthermore, Urban Gallery links stakeholders and actors to issues of globalization and ongoing social, economic, and ecological processes which can facilitate change, and brings the methodology beyond the "small scale, bottom-up, and eco-friendly moves" (Shane 2004: 7) by its focus on cultural urban mapping as dynamic and emergent but at the same time located in a systemic view of the built and the lived beyond the immediate and discrete. Users are prompted to link everyday and mundane objects and artifacts of their built environment to wider cultural and productive processes and origins of production and exchange, that is as knowledge production, so that experiences are connected to thinking transformationally and not merely mapping by fixed category. This makes the tool particularly relevant in the education of architects to work in complex and fluid urban situations, as it suggests a design approach beyond the technological and object oriented approach to design, which lies at the core of the profession.

\section{Context and application}

The aim behind designing the Streetscape app was to make use of digital devices and the apparent and potential affordances of mobile locative media to develop explorative mapping tools that played up to, and were in close concordance with, both vital forces of urban change and common modes of seeing and experiencing the city. Based on insights from both postmodern approaches to mapping and poststructural perspectives on knowledge production our intention was to explore the productive capacities in combining the digital technologies of locative media with more experimental and strategic forms of urban mapping as in the Urban Gallery methodology. This was more than a mere transposition of approaches to smartphones and GPS-based markers.

Our overall design guideline was to see mapping as an active mode of interpretation (and not as a passive form of reproduction), i.e., as a way of engaging with urban environments. Accordingly, the co-design process (in which the design and development and the teacher-researcher teams' competences in urbanism, media, informatics, communication, and interaction design were represented) was set up so that insights into the creative affordances of both technical (and communicational) features and urban use contexts could be reciprocally adjusted throughout the design process. They then were also optimized in the final design of the app, such as the move to higher image resolution (Morrison et al. 2012a). 


\section{Streetscape}

We chose the first stages of the Urban Gallery methodology as the basis for our design work, and named the app after the "street-level ethnography" (Corner 1999: 243) it includes. Streetscape was based on the apparent similarities between the Urban Gallery methodology and NarraHand, a mobile collaborative and GPS-based narrative app developed by members of the research team (Mainsah and Morrison 2011; Morrison and Mainsah 2012; Morrison et al. 2012b). First, locative annotation was common to both, and second, so was the narrative post-production following the actual locative mapping in the street (in NarraHand in the form of a net-based wiki). This wiki aspect that was designed to allow users to reflect on and critique their initial observations made through use of the tool was not unlike the use of paper-based multi-person scenarios in the Urban Gallery. The use of the Streetscape app described in this chapter focused on students carrying out mapping in the field, and experiencing the collaborative prototype development potential of the database through the wiki, while the additional stages of the Urban Gallery (scenario game and action plans) were not included.

Users of Streetscape are required to log into the app using one of the four perspectives Erasure, Origination, Transformation, or Migration (E, O, T, or M). From one of these perspectives, any point on the map can be annotated with photo, text, and tags, and any new entry becomes immediately shared and visible to other users

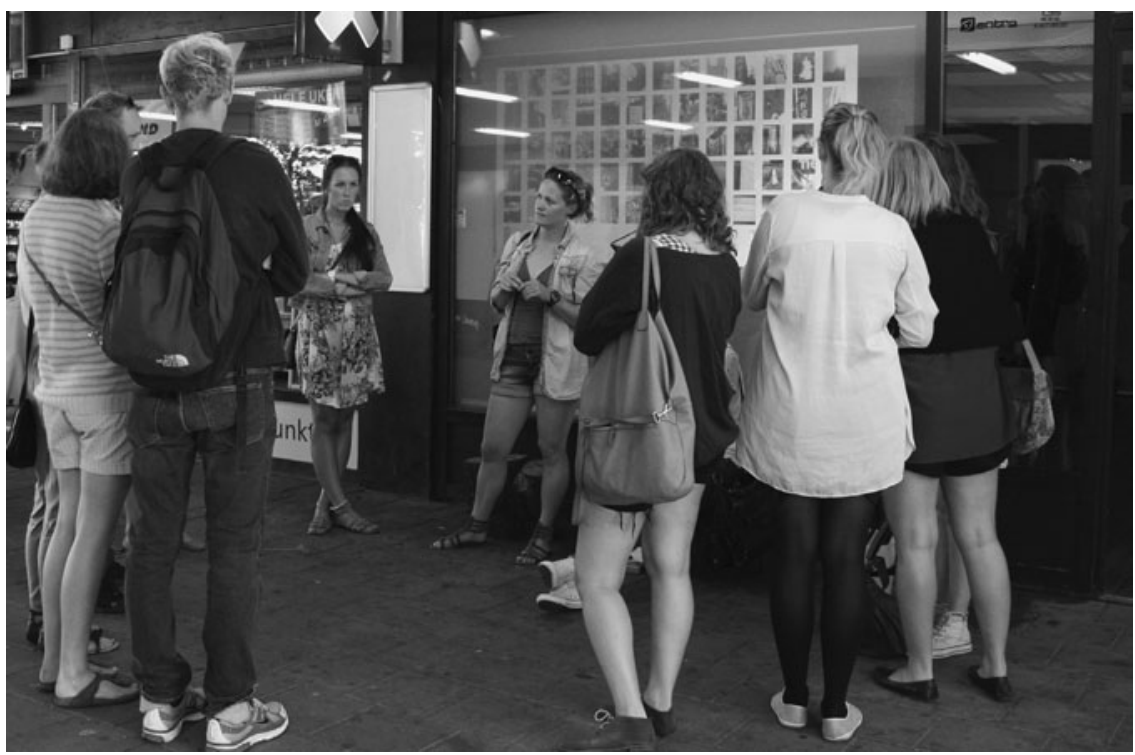

Figure 10.I Mapping results were displayed real-time in a local shop window (photograph by the YOUrban project). 
on the map. It is also possible to prepare "sample points" in advance, and these have a different visual appearance, that change as users navigate to them and annotate them using their designated perspective. In the app it is possible to search for both nearest and most recent entries, and existing entries can be modified by other users. In the associated web wiki, annotated points appear in lists, and new entries with tags associating them to other sample points can be added.

Streetscape was tested in a two-day workshop with sixty students of architecture in August 2011 in a local area of Oslo called Tøyen. It was organized in collaboration with a local arts and urbanism collaborative and used a shop window in the local shopping center to publicly display live mapping (Figure 10.1). This area of the city was chosen because it is one of the most socially challenged parts of urban Norway: It has high numbers of immigrants, low levels of people with higher education, and a high level of unemployment. Architecture and urban design needs to develop ways of working in these contexts to make it relevant for people in such areas. We involved students in applying Streetscape on the streets of this part of our city. The research design team observed lively dialogue in the groups; students shared mobile devices and participation in making entries was high.

Day one of the workshop was devoted to mapping activities in the field using the Streetscape app installed on iPhones and one iPad. The students worked in sixteen groups, and they visited randomly given points in the neighborhood

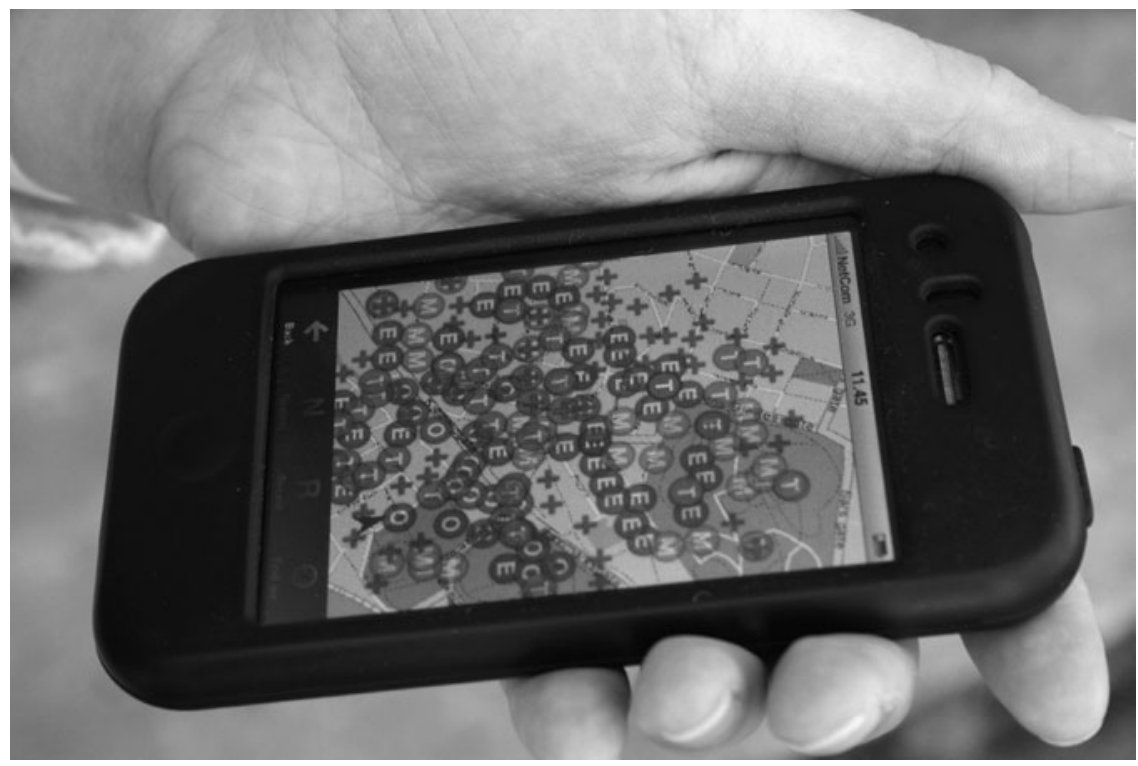

Figure 10.2 The map changed interactively as mapping was conducted (photograph by the YOUrban project). 
(Figure 10.2). Students used the relatively coarse application map and the "Direct me there" function for navigation. In the original Urban Gallery the term "bean drop" refers to the actual random selection of sites by dropping a large number of dried beans on a paper map; in the Streetscape version we replaced it with the term "sample point" which has a natural science connotation and involved us clicking randomly over the selected screenspace. Students used the integrated navigation tool to navigate to the sample spots, and made an observation of a physical feature, a social encounter, a view, an ephemeral event, or something entirely different, in which the designated perspective could be observed and described. When annotated, the graphical markers on the map changed to (E)rasure, $(\mathrm{O})$ rigination, $(\mathrm{M})$ igration, or $(\mathrm{T})$ ransformation. The participants made observations according to the designated perspectives, took photographs, annotated the sample points with texts and added labels (see Figure 10.3).

Texts and photos of sample points were ported to a wiki for reflection on mediated urban contexts, and on day two, students developed scenarios using both the wiki and a paper-based format; these were then presented in plenary and saved to the wiki. In one case students noticed chalk lines on the grass in a public park that seemed to indicate an imaginary building (Figure 10.3). Another group of students interviewed a mushroom collector in another location, and

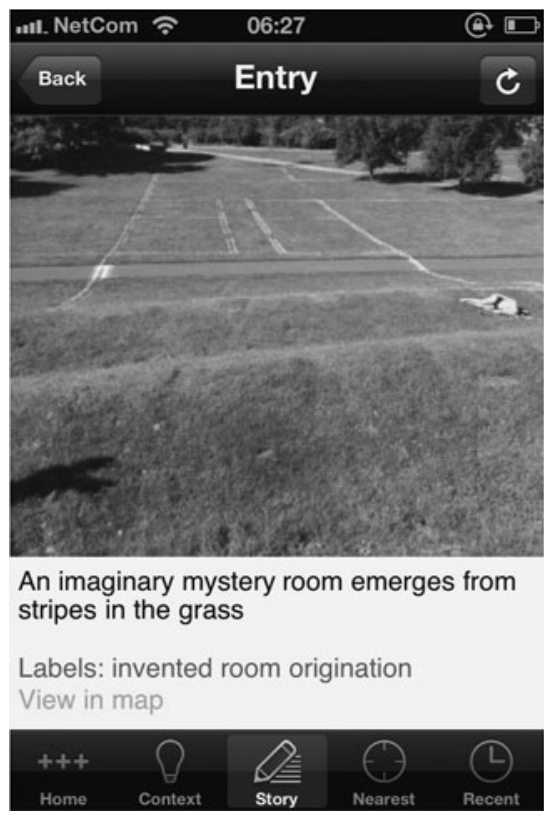

Figure 10.3 Example of sample point notation by students (screenshot by the YOUrban project). 
joined with the first observation the idea of what they called a "mushroom academy" - a building and an associated institution to teach people to differentiate between edible and poisonous fungi-was proposed on the second day of the workshop, a concept that could easily be associated with actual physical space, real funding opportunities, and actual actors with aligning interests.

The workshop formed part of a curriculum in which students were introduced to cultural urban mapping, and particularly the Urban Gallery method, and the students' work was small-scale design experiments, which were important in evaluating the relationship between the selected mapping approach and the development and functionality of the app. The basic research mode was that of a qualitative inquiry in which the research and design team observed students using the app in the field using selected film documentation of group dynamics - both within the teams, and within the group as a whole. Two groups of four students were interviewed about the perceived relevance of the urban mapping approach as well as the experience of using a designated mapping app and associated wiki. A written evaluation using a short electronic questionnaire covering the same issues was conducted, and one final plenary evaluation session was held where students' views were discussed with the design research team.

\section{Outcomes}

We were still interested in looking into how acts of urban mapping are influenced when transposed from an existing paper-based mode to a mobile app. We found that the mapping method of Urban Gallery was effectively transposed to mobile, social, and locative media through the app, which enabled collective production and sharing of information. In the transposition changes and simplifications were made, including a reduction from four to one dynamic perspective on each site (for details, see Morrison et al. 2012a). New aspects in the mapping procedure also emerged as result of the transposition.

The app was designed to be used in contexts as a device for producing emergent and location-based new readings of urban social and physical spaces via mobile activity and in respect to four broad perspectives. Though the overall context of use, as in Urban Gallery, initially has been to open out diverse views on local urban situations and potentially different social and architectural practices, the Streetscape app could tentatively be put into use in a variety of contexts in which creative, cultural mapping of urban features (of various kinds) is on the agenda. This is important for students of architecture who need to see links between their own uses of social media and their processes of understanding how professionally informed yet participative urban mapping applications may be taken up productively in contexts of culture and communication by experiencing how observations can be connected with abstract forces and agents driving the development of urban form and related planning.

In our urban, developmental, design and learning context, the app was trialed and tested by a specific group of experts (students of architecture) with a specific 
purpose in mind. This marks it out as a "collaborative project" (Kaplan and Haenlein 2012) in which participants contributed to a database that was then shared and integrated into views of how the given area of the city might be changed through interventions in stage two of the workshop. Importantly, participants were in "competition" to apply their perspectives to the designated sample points; they could change the contents of already mapped spots, in which case the spot would be remapped according to the new perspective (and the screen icon would change). Collective authoring was thus an issue both in relation to the actual mapping activities in the field, as well as in the second stage of creating narratives using a wiki.

Literature on ubiquitous computing and locative media deal with the mix of or interrelation between media information space and physical space (Shepard 2011; Gordon and de Souza e Silva 2011). While onsite information retrieval is not a primary function of Streetscape, it is a possibility that emerges from the geolocated networked technology (such as Foursquare or Gowalla). This may add an important new layer to the methodology, changing the mapping aspect by enabling real-time co-production of narratives, in which all mappers interactively inform each other as mapping takes place. In other words, the co-narration of urban space is now directly connected to the site as a possible locally retrieved aspect of space. Local and remote users may produce and retrieve observations and narratives in a dynamic effect, resulting in new

conditions of physical spaces. To be local means to be engaged with the local conditions of a physical space, whether the user is physically proximate or not. This is a remarkable shift in how we understand urban space and one that becomes more remarkable when we confront the issue of co-presence.

(Gordon and de Souza e Silva 2011: 55)

We were interested in the outcomes in terms of mapping procedures and contents, and what the potential for further development of an urban mapping app might be. In testing Streetscape we found that:

- the app helped students map the city, discovering new urban specifics and place-related qualities in a probing and investigative way (Figure 10.4). The set-up of the app itself seemed to spur or motivate students;

- phenomena observed and mapped appeared to roughly parallel those mapped in the paper-based versions of Urban Gallery. An emphasis seemed to be on physical phenomena, such as buildings, vegetation, and urban surfaces, while processes primarily involving people in social situations were less prevalent;

- combining map media specifics, GPS navigation, and assigned mapping tasks spurred students' imagination and attention. In particular, the combination of a fairly abstract map and GPS navigation proved more fruitful than expected; and 


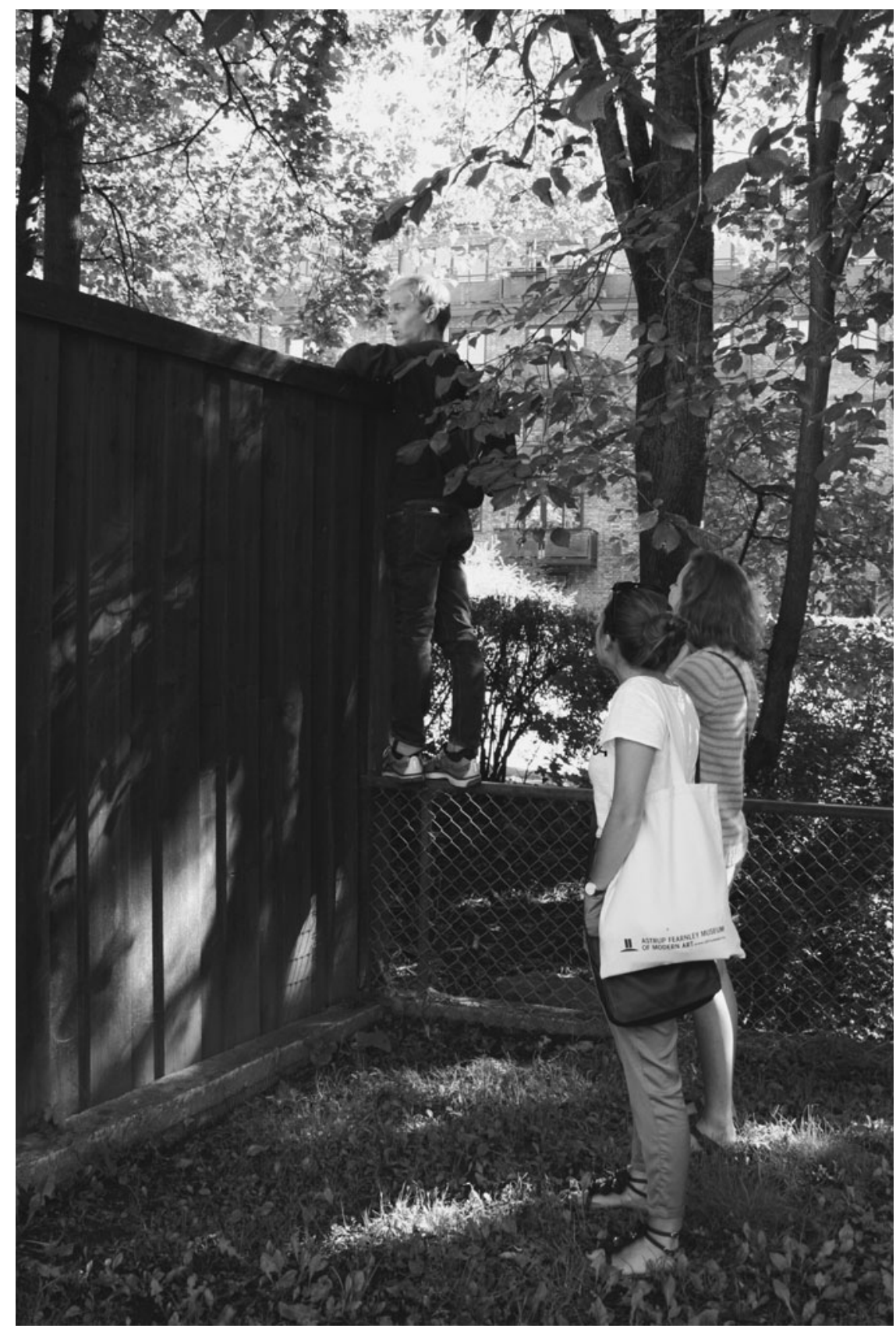

Figure 10.4 Use of the app led students to see the city in a new way (photograph by the YOUrban project). 
- students reported in the evaluation that using the app made them see environments differently and discover new kinds of features and qualities.

Since its uses mentioned here we have introduced Streetscape into a course for a group of Norwegian and international students of architecture. Once familiar with the tool and the types of activities reported here, the students were asked to pitch creative alternatives to the tool, extending their understanding of it to their own interests and contexts of cultural mapping, for example in an online discussion between fellow students in Mexico City and Tallinn in Estonia. Evidence from these applications of Streetscape indicates great potential for further development of experimental urban mapping, street-level urban ethnography, and the use of handheld devices for initializing processes of participation and bottom-up surveying of urban resources. The fully functioning app is available on the iTunes App Store as a read-only version, and we have now launched a second release as an open, public version with more specific mapping capabilities and an interface with external geolocated social media contents called MAPPA. We have added Facebook log-in functionality and the option for interest groups to apply their own research objectives and perspectives. This will allow the app to shift from a development and trialing phase to a context of active locative, mobile cultural mapping and related multimodal communication.

\section{Discussion}

Our initial idea was that the experimental urban mapping practices of Urban Gallery might provide input to the development of new and more place-sensitive ways of programming locative media applications. This was both in terms of adding procedures that could stimulate more explorative readings of the specifics of urban environments, and of exploiting unused potentials of emerging locative media application technologies. Students reported that using the app made them experience the city in new ways, and the interactivity and dynamic perspectives embedded in the app indicated a potential for entirely new forms of digitally collaborative narratives for the city.

In our study we envisaged that by making use of assets of social and locative media technologies one could add new modes of feasibility and content to experimental urban mapping methodologies. This includes, particularly, the ability to not only read but also write the city, an affordance of locations-based services, as pointed out by Shepard (2011), but also bringing it beyond mere writing to collectively "reformulating what already exists" (Corner 1999: 214), enabling "the re-shaping of the worlds in which people live" (Corner 1999: 213).

In addition, we were motivated to point to ways that locative and social media (Gordon and Manosevitch 2011) through use might inform discourses on urban planning, governance, and design (Gordon and Manosevitch 2011) in which perspectives on the more imaginative and transgressive qualities of cities and urban living are still fairly limited. Experiences from the actual uses of the app 
revealed forms of interactivity that emerged between users while mapping, but also potentially between locative social media and the mapping subject in urban space. This is indicative of a reading of the city that moves beyond the individual perspectives of Lynch (1960) and Jacobs (1961), to one that provides some basis for more innovative and immersive ones - enabled through locative affordances embedded in smartphones and the designed app, together with the capacity for collaborative and spatial exploration and annotation-but also related critical and reflexive turns toward urban planning.

As mentioned earlier, in the existing literature we have found little extended exchange between ongoing research in locative media and place-specific computing that involves ubiquitous technologies and their relations to mapping and the design of cities (e.g., Speed 2010; Shepard 2011). We see a need to remediate this in a sense: Streetscape introduces the locative into the planning and cultural mapping sphere: it uses the affordances of technology through collaborative features and by way of access to postings by other participants. The screenspace is reflexively productive: In Streetscape, productivity is looped back into physical locations and placement in the built environment, such as the projections of mapping entries on the urban screen located at the local shopping center.

The use of locative social media in urban mapping, as indicated by the application of technology discussed in this chapter, reinforces a multi-layered reading of urban space, pointing to the fact that "mapping is a fantastic cultural project" (Corner 1999: 213). It is important to investigate further, we would argue, how to make the mediated city legible through developing tools for further expanding our mobile literacies. However, these should not just be ethnographically inspired legitimations of ubiquitous computing (Dourish and Bell 2011). What is needed are tools that permit and enable locative enactments that enhance understanding of urban life by moving beyond functionalism and technological determinism toward constructing and situating technologies in relation to cultural settings and practices (e.g., Wagner et al. 2010). Attention to the design of such tools is critical as we can only be as expressive and communicative as the affordances these tools enable.

\section{Conclusion}

We envisage the networked city as a technologically and culturally complex context that in increasingly intricate ways informs and remediates everyday urban living, social interactions, and how the city is used and perceived. The overall intention behind developing Streetscape was to make proactive use of the digital affordance of the emerging networked city in order to create a cultural mapping tool that allows people to actively engage in and with their urban environments and thus see, read but also write, the city and its many complex features in creative and perhaps even unexpected ways. As city dwellers-as urbanists and interaction designers too-we still currently lack elaborated and sophisticated representational means and forms for perceiving the networked 
city. We hope that our experiential urban mapping tool, as well as the revised and more versatile mapping app called $M A P P A$ will contribute to the development of a creative and critical discourse on the complexities of the digital city. From design and through experience we see that there is room in the "streetscapes" of the networked city to increase our cultural imagination by using locative social media as means both for new and inventive forms of cultural mapping of the city.

\section{References}

Albrechts, L., P. Healey, and K. Kunzmann (2003) Strategic spatial planning and regional governance in Europe, Journal of the American Planning Association, 69(2): 113-129.

Allen, S. (2000) Practice: Architecture, Technique and Representation, Amsterdam: G+B Arts International Imprint.

Amoroso, N. (2010) The Exposed City: Mapping Urban Invisibles, Abingdon: Routledge. Anker, N. E. and P. Anker (2011) Viewing the earth from without or from within, in E. Jazairy (ed.), New Geographies 4: Scales of the Earth, Cambridge, MA: Harvard Graduate School of Design, pp. 89-94.

Awan, N., T. Schneider, and J. Till. (2011) Spatial Agency: Other Ways of Doing Architecture, Abingdon/New York, NY: Routledge.

Bull, M. (2005) The intimate sounds of urban experience: An auditory epistemology of everyday mobility, in K. Nyíri (ed.), A Sense of Place: The Global and the Local in Mobile Communication, Vienna: Passagen Verlag, pp. 169-178.

Bunschoten, R. (1997) Black Sea: Bucharest stepping stones, Architectural Design Series: Architecture after Geometry, 67(5/6): 82-91.

Bunschoten, R. (2001) Urban Flotsam, Rotterdam: 010 Publishers.

Bunschoten, R. (2007) Scenario games. Vital techniques for interactive city planning, in F. von Borries, S. Walz, and M. Böttger (eds.), Space Time Play. Computer Games, Architecture and Urbanism: The Next Level, Basel: Birkhäuser Publishing, pp. 384-387.

Bunschoten, R. (2010) Urban prototypes, in M. Mostafavi and G. Doherty (eds.), Ecological Urbanism, Baden: Lars Müller Publishers, pp. 616-621.

Calthorpe, P. (1993) The Next American Metropolis: Ecology, Community, and the American Dream, New York, NY: Princeton Architectural Press.

Campbell, T. (2012) Beyond Smart Cities. How Cities Network, Learn and Innovate, Abingdon: Routledge.

CHORA (2010) Taiwan Strait climate change incubator, in M. Mostafavi and G. Doherty (eds.), Ecological Urbanism, Baden: Lars Müller Publishers, pp. 622-627.

Corner, J. (1999) The agency of mapping: Speculation, critique and invention, in D. Cosgrove (ed.), Mappings, London: Reaktion Books, pp. 213-252.

Cosgrove, D. (1999) Introduction: Mapping meaning, in D. Cosgrove (ed.), Mappings, London: Reaktion Books, pp. 1-23.

Costanza-Chock, S. (2012) Mic Check! Media cultures and the Occupy Movement, Social Movement Studies: Journal of Social, Cultural and Political Protest, 11(3/4): 375-385.

Crawford, A. (2008) Taking social software to the streets, Journal of Urban Technology, 15(3): 79-97. 
de Certeau, M. (1984) The Practice of Everyday Life, Berkeley, CA: University of California Press.

de Souza e Silva, A. and J. Frith (2012) Mobile Interfaces in Public Spaces: Locational Privacy, Control, and Urban Sociability, Abingdon: Routledge.

Dourish, P. and G. Bell (2011) Divining a Digital Future, Cambridge, MA: MIT Press.

Forester, J. (1989) Planning in the Face of Power, Berkeley, CA: University of California Press.

Gordon, E. and A. de Souza e Silva (2011) Net Locality: Why Location Matters in a Networked World, Chichester: Wiley.

Gordon, E. and E. Manosevitch (2011) Augmented deliberation: Merging physical and virtual interaction to engage communities in urban planning, New Media \& Society, 13(1): 75-95.

Gordon, E., S. Schirra, and J. Hollander (2011) Immersive planning: A conceptual model for designing public participation with new technologies, Environment and Planning $B, 38(3): 505-519$.

Graham, S. and S. Marvin (2001) Splintering Urbanism. Networked Infrastructures, Technological Mobilities and the Urban Condition, London: Routledge.

Harley, J. (1989) Deconstructing the map, Cartographica, 26(2): 1-20.

Harvey, D. (1996) Justice, Nature and the Geography of Difference, London: Blackwell.

Healey, P. (1992) Planning through debate: The communicative turn in planning theory, Town Planning Review, 63(2): 143-162.

Hjorth, L., J. Burgess, and I. Richardson (eds.) (2012) Studying Mobile Media: Cultural Technologies, Mobile Communication, and the iPhone, Abingdon: Routledge.

Innes, J. and D. Booher (2004) Reframing public participation: Strategies for the 21st century, Planning Theory \& Practice, 5(4): 419-436.

Ito, M. (2009) Hanging Out, Messing Around, Geeking Out: Kids Living and Learning with New Media, Cambridge, MA: MIT Press.

Jacobs, J. (1961) The Death and Life of Great American Cities, New York, NY: Random House.

Kaplan, A. and K. Haenlein (2012) Social media: Back to the roots and back to the future, Journal of Systems and Information Technology, 14(2): 101-104.

Lefebvre, H. (1991) The Production of Space, London: Blackwell.

Lemos, A. (2010) Post-mass media functions, locative media, and informational territories: New ways of thinking about territory, place, and mobility in contemporary society, Space and Culture, 13(4): 403-420.

Lynch, K. (1960) The Image of the City, Cambridge, MA: MIT Press.

Mainsah, H. and A. Morrison (2011) African clouds over the Oslo opera, Computers and Composition, 28(3): 235-245.

Messeter, J. (2009) Place-specific computing, International Journal of Design, 3(1): 29-41.

Morrison, A. and J. Aspen (2013) Building appetites: The design of locative media apps for learning the networked city, in Proceedings of DRS/CUMULUS 2013. 2nd International Conference for Design Education Researchers, Oslo, May 14-17.

Morrison, A. and H. Mainsah (2012) Building communication by design: Mobile fiction and the city, in M. Hensel (ed.), Design Innovation for the Built Environment: Research by Design and the Renovation of Practices, Abingdon: Routledge, pp. 221-234.

Morrison, A., J. Aspen, P. Hemmersam, I. Sem, and M. Havnør (2012a) Designing experimental urban mapping with locative social media, Proceedings of the Design Research Society 2012: Bangkok (CD-rom). 
Morrison, A., J. Aspen, and E. Westvang (2013) Making the mobile and networked city visible by design, in Proceedings of Crafting the Future, 10th European Academy of Design Conference, April 14-17, available at: www.trippus.se/eventus/ userfiles/39130. pdf.

Morrison, A., H. Mainsah, I. Sem, and M. Havnør (2012b) Designing location-based mobile fiction: The case of NarraHand, in R. Jones (ed.), Discourse and Creativity, New York, NY: Longman, pp. 211-230.

Nielsen, T. (2008) Gode intentioner og uregerlige byer (Good Intentions and Unruly Cities), Aarhus: Arkitektskolens Forlag.

Pop, S., G. Tscherteu, U. Stalder, and M. Struppek (2012) Urban Media Cultures: (Re) Shaping the Public Space through Urban Screens and Media Architectures, Ludwigsburg: avedition.

Proboscis (2004) Urban Tapestries Context, online movie, available at: http://research. urbantapestries.net/films/UTContexts_sml.mp4.

Proboscis (2006) Social Tapestries, online movie, available at: http://socialtapestries.net/ outcomes/Social_Tapestries_2006.mov.

Proboscis (2007) Social tapestries projects, available at: http://socialtapestries.net/ projects/.

Rainie, L. and B. Wellman (2012) Networked: The New Social Operating System, Cambridge, MA: MIT Press.

Roberts, L. (2012) Mapping cultures: A spatial anthropology, in L. Roberts (ed.), Mapping Cultures: Place, Practice, Performance, London: Palgrave Macmillan, pp. 1-28.

Shane, G. (2004) The emergence of "landscape urbanism": Reflections on Stalking Detroit, Harvard Design Magazine, 19: 1-8.

Shepard, M. (ed.) (2011) Sentient City: Ubiquitous Computing, Architecture, and the Future of Urban Space, Cambridge, MA: MIT Press.

Speed, C. (2010) Developing a sense of place with locative media: An "Underview Effect," Leonardo, 43(2): 169-174.

Speed, C. and J. Southern (2010) Handscapes-Reflecting upon the use of locative media to explore landscapes, in E. Buhmann, M. Pietsch, and E. Kretzler (eds.), Proceedings of Digital Landscape Architecture 2010, Anhalt University of Applied Sciences, Berlin: Wichmann, pp. 164-172.

The New York Times (2012) The dawning of domestic drones, New York Times, December 25, available at: www.nytimes.com/2012/12/26/opinion/the-dawning-of-domesticdrones.html? $\mathrm{r}=1 \&$.

Wagner, I., T. Bratteteig, and D. Stuedahl (eds.) (2010) Exploring Digital Design, Vienna: Springer.

Wood, D. and J. Fels (1992) The Power of Maps, New York, NY: Guilford Press.

Yaneva, A. (2012) Mapping Controversies in Architecture, Farnham: Ashgate. 


\title{
Designing for mobile activities \\ WiFi hotspots, users, and the relational programming of place
}

\author{
Michael R. Doyle
}

The use of information and communication technologies (ICTs) was once limited to particular places. Phone calls were made from home, the office, or from phone booths. Access to the internet was first introduced in the office and then entered the home during the 1990s with the marketing of the personal computer and online services. Technologies were bound to places and bridged the distances between them. For the individual ICT user, two places, the home and office, were the two main poles of technology use.

With the rapid proliferation of mobile technologies at the beginning of the twenty-first century, ICTs no longer connect places, but individuals (Wellman 2002). Portable devices and the increasing ubiquity of telecommunications and wireless internet (WiFi) networks allow (and sometimes pressure) individuals to conduct a greater number of activities in a variety of places. The home and office are losing their status as the preeminent locations of mediated information access and communication.

The observation of several parallel trends both raised concern and inspired celebration about a cyberspace deemed separate from the physical places of the city. On the one hand, the globalization of the flows of capital and labor were declared to have disconnected social and economic production processes from space and time (Sennett 2005). ICTs were blamed for having homogenized the experience of place (Meyrowitz 1985), evacuated third-places of their social importance (Oldenburg 1989) and increased social isolation (Putnam 2000). On the other hand, portable computers and videoconferencing were thought to have rendered face-to-face exchanges a thing of the past (Amin and Thrift 2002; Graham and Marvin 1996), liberating the individual from spatial and temporal constraints (Benkler 2006). ICTs were praised for their ability to "reactivate" public spaces as places of work and leisure (Mitchell 2003).

Neither extreme has come to pass. What we are left with is an emerging understanding of places as composed of varying degrees of materiality and immateriality. They are neither entirely private, nor entirely public, but somewhere in between (Sheller and Urry 2003). They are inseparable from the people who visit them (Sheller and Urry 2006). With the rise of location-based media, this information may be connecting them more with their immediate 
environment, than taking them away from it (Gordon and de Souza e Silva 2011). Evidence suggests that internet users are even more likely to visit public places than non-users (Hampton et al. 2009). Whether they are mobile by choice or obligation, mobile individuals look for places to go. Where they end up may depend as much on the physical qualities of the place as on the number of friends who have checked in there.

The growing emphasis placed on location by ICT use has done little to raise a debate among architects and urban planners about the implications that emerging spatial and technological practices may have for their role in a network society (Castells 2000). The ability to conduct an activity either at home, at an office, on a park bench or at a café questions the urban and architectural tradition of assigning predetermined activities to spaces. Rather than ignorance, this may be due to a certain level of ambivalence: Both the dystopian and utopian technological discourses imagine a future where, for better or for worse, physical place is of little importance. Furthermore, the material reality of mobilities, which is situated somewhere between the rationalization of movement flows and the localized tactics of the average urban pedestrian, is not yet properly seen as having design potential (Jensen 2013).

This chapter takes a novel look at place-making through ICTs by examining the places of the city where mobile technology users come to rest. First, I discuss the influence of mobile technology on travel and on the importance of context. Then I present a study conducted from 2008 to 2010 in Québec City, Canada, of WiFi users and hotspots in collaboration with a local network provider, ZAP Québec, looking to better understand its clientele. Québec City is an interesting case because the expansion of the WiFi network has received city government and citizen support since its inception in 2006 (Therrien 2009), and was even part of the Liberal Party election platform at the launch of this study in 2008 (Élections Québec 2008). The results suggest that, although WiFi users do not fit a single profile, the public places where WiFi use is most frequent share certain urban and spatial qualities. In the conclusion I discuss the relevance of these findings for architecture and urban planning in a mobile world.

\section{Mobile people, devices, and activities: the absence of the built environment in recent studies}

Mobile technology use in urban places was approached in this research project as a phenomenon combining both physical movement and ICT. For a good part of the twentieth century, ICT use and travel were viewed as substitutes (Graham 1998; Schwanen and Kwan 2008). It was presumed that the ability to make a phone call or access information over the internet would reduce or eliminate the need to travel, resulting in a decrease in traffic congestion and in greenhouse gas emissions. This hypothesis was met with skepticism and by the early twenty-first century more evidence had been produced suggesting that ICT use and travel are 
complementary (Mokhtarian 2002) and that their different articulations create previously unobserved space-time geographies (Couclelis 2004).

Empirical research since the mid-2000s has investigated the emerging forms of mobility that this complementarity suggests. Several studies investigated correlations between specific technologies and travel and activity behavior. Cell phone use, for instance, was found to be associated with a greater number of outof-home activities (Lee-Gosselin and Miranda-Moreno 2009), a greater tendency to make trip changes while in route (Srinivasan and Raghavender 2006), a higher use during commute times (Afanasyev et al. 2008), and with a greater number of daily trips (Thulin and Vilhelmson 2007) and social contacts (Licoppe et al. 2008). Smartphone penetration was regrettably limited at the time of many of these studies, but a study conducted in the San Francisco area did observe a higher use of the internet on smartphones during commute times (Afanasyev et al. 2008).

These studies raise questions as to where people using mobile technologies go and what they do. For instance, when investigating "activity fragmentation"the conducting of single activities across various places and times (Couclelis 2004) - Lenz and Nobis (2007) observed that cell phone and laptop use correlated highly with activity fragmentation. The activities were considered fragmented if the same task (like writing a paper or email) had occurred in multiple places (usually the home, the office, or a public space). This study, like many of the others, did not specifically investigate the types of places into which these activities were being fragmented.

A series of qualitative and mixed-method studies shed light on the places where mobile technology use can occur. These studies specifically looked at professional work and how multiple spaces are defined as different types of workplaces. ${ }^{1}$ For instance, mobile workers tend to come to the office for face-toface communication (Brown and O'Hara 2003), while the home can be a place for intense periods of concentration (Hislop and Axtell 2009) and the car (particularly in traffic) a place to catch up on phone calls (Laurier 2004). Where ICT was used and what activities were performed depends mostly on the amount of time spent traveling (Lyons et al. 2007) and the ease with which devices can be used (Axtell et al. 2008).

These studies attest to the importance of context in mobile technology use. The social and physical properties of a place - as well as those frequented before or after in a sequence (Brown and O'Hara 2003) — play an important role in facilitating or hindering the ability to conduct particular activities on a mobile device. In the case of free wireless internet access (WiFi), the individual could frequent any number of places and reasonably expect the ease of connecting to be similar. Laura Forlano's (2008) interviews with New York City WiFi users reveal some of the other aspects that could come into play such as the ease of finding a place to sit, the number of outlets, or the proximity of the hotspot to the home or the office. For example, one of her interviewees frequents a café located in a bookstore when he wants to reference books for his work, spends some of 
his time at another where he has room to spread out and get feedback from friends and acquaintances he meets there, and, when he needs to concentrate, travels across town to another café where he is anonymous and can work uninterrupted. Whereas the social aspects of the spaces receive a lot of attention in WiFi research (see also Gupta 2004), the physical qualities of these spaces have yet to be explored.

Contributions from architects and interaction designers provide some evidence about where the importance of context on mobile technology use may take architecture and urban planning. If on the one hand architecture's fascination with nomadism has inspired imagined cities where individuals travel in techheavy self-sufficient infrastructural pods like Archigram's 1964 Plug-in-City and The Walking City, today's nomad needs only a mobile device to connect and disconnect on the go (see Abbas 2006). In a world of cloud computing, WiFi, and high-speed cell-phone data transfer, the necessary infrastructure is embedded within the environment. Worker mobility has received quite a bit of interest as mentioned previously and office design has awakened to the fact that their employees might not need to be in the office to get work done (Duffy 2008; Nicolaou 2006). The result so far however has been more to import into the workplace objects of play (slides, game rooms, etc.) and of domesticity (couches, lounge chairs) than to propose design alternatives on a city scale or to question existing building typologies.

The questions faced by architecture and planning are shared by interaction design. The increase in location-based mobile technology applications means that place becomes more important as technology use responds to the immediate context (Aurigi and De Cindio 2008). Interaction design's emphasis on designing experiences rather than objects could inspire architecture to do the same, moving architecture, like technology, into the background as enablers of events (McCullough 2004). Architectural historian and engineer, Antoine Picon (2010) reminds us that, like cell phones, which rely on an underlying infrastructure, buildings depend upon various infrastructures to remain usable and accessible. Despite their apparent permanence, buildings and neighborhoods are implicated within networks of various scales. The more they are disconnected from this network, the less they can be adapted to changes in use.

Although these authors insist upon the important contribution that context should make to the design of buildings and mobile technologies, they do not provide answers to the questions raised by the empirical literature, which has not specifically focused on the places of ICT use. This chapter bridges this gap by investigating the use of free wireless internet (WiFi) in Québec City, Canada, where the WiFi network has been steadily growing since 2006 and where wireless internet use outside the home and office has been on a steady rise provincewide since 1999 (CEFRIO 2008). WiFi is particularly interesting because it associates internet access with a particular place, reinforcing the idea that mobile technology is as much about finding a place to stop as it is about movement (Mackenzie 2010). 
What information could aid ZAP Québec in orienting the planning of its network expansion? Which WiFi hotspots are frequented the most and what spatial qualities do they share? If the empirical studies suggest that most ICT users are educated males under the age of thirty-five, who else is using ICT? What are they doing remotely with mobile technology? How can the answers to these questions guide the choice (or design) of future WiFi hotspots? The following section describes the three methods used in responding to these questions.

\section{Investigating WiFi hotpots and users in Québec City}

Three principal explorations comprised the research project, which benefited from a close collaboration with the local non-profit provider, ZAP Québec. ${ }^{2}$ First, an internet survey was conducted of individuals connecting at any one of ZAP Québec's 144 hotspots. The questionnaire (a link to which was placed on the splash pages users saw when connecting) asked questions (in French) about the places and times of their WiFi use, the reasons they chose the hotspots they frequented the most, and what they did there. In order to glean information about their temporal and spatial practices, it asked about the flexibility of the work schedule and the travel modes they used. A series of questions addressed household structure, professional status, and income.

Parallel to this first step, the study looked at the frequency at which the WiFi hotspots managed by ZAP Québec, which include cafés, bars, restaurants, libraries, community centers, and ice rinks, had been used since the launch of the network in 2006. The data were provided by ZAP Québec in three-month intervals from January 2007 to March 2010 and contained the number of unique visits received by each hotspot. The most frequented hotspots were identified by calculating the average number of visits per day since the opening of each hotspot and mapped using GIS software. Mapping aided in situating the hotspots within a wider urban context and revealed some of the characteristics of their surroundings.

The study concluded with a spatial analysis focused specifically on the hotspots that were the most frequented or that had been identified as the most desirable by the survey respondents. The analysis compared the spatial layout of the hotspot and its immediate surroundings with spatial configurations derived from the pattern language conceived by Christopher Alexander and colleagues in the 1970s (Alexander et al. 1977). Each pattern refers to a specific combination of built and natural elements that are thought to be a sort of common denominator in the built environment. ${ }^{3}$ The following section presents the study's principal findings, first from the internet survey, then from the mapping of the most frequented hotspots and concludes with findings from the spatial analysis. 


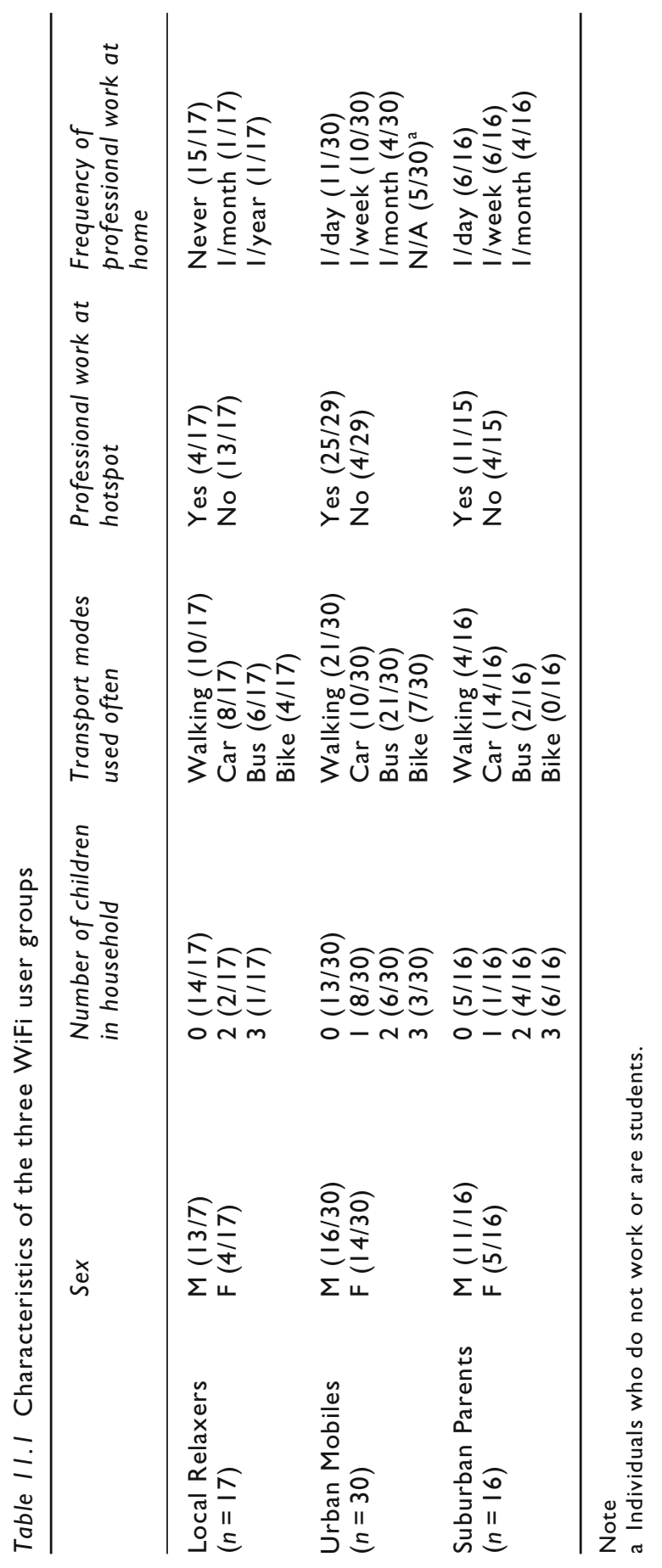




\section{WiFi hotspots and users: everyday places and people}

\section{WiFi users: not just young "techies" but retirees and parents as well}

Contrary to recent studies that characterize the ICT user as a well-educated male in his mid-thirties (Forlano 2008; Lenz and Nobis 2007; Lyons et al. 2007; LeeGosselin and Miranda-Moreno 2009), this particular profile only represents about one-fifth (fourteen of sixty-three) of the respondents in Québec City. Several WiFi users are over the age of thirty-five (fourteen of sixty-three) and a smaller number (eight of sixty-three) over the age of fifty-five. There is an overrepresentation of respondents between the ages of eighteen and twenty-four, possibly due to several of the hotspots being located nearby schools and universities and therefore attracting more students. There are, however, several retirees among those over the age of fifty-five. The proportion of males to females is about two to one (forty to twentythree), which is similar to Laura Forlano's (2008) findings in her survey of WiFi hotspot users in Montréal. Participants with at least an undergraduate diploma outnumber those with less education by two to one (forty-two to twenty-one).

The sixty-three respondents frequent hotspots for WiFi use throughout the day on both weekdays and weekends. In general, they spend anywhere from thirty minutes to four hours conducting activities such as writing and reading email, searching for information, chatting online, listening to music, and shopping. Hotspots are frequented at various times of the day, with a slightly higher tendency to go in the evenings and outside of the meal times (when hotspots such as restaurants and cafés are the busiest).

Interestingly, the full-time workers in the sample go to hotspots during regular business hours as often as students do, whereas traditional nine-to-five workers would be presumed to have been present mostly during the evening and meal-time hours. This is perhaps less surprising if we consider that the full-time workers in the sample are mostly educators and computer technicians, and are self-employed or consultants - a group whose spatial and temporal flexibility may be similar to that of students. For over two-thirds (thirty-four of forty-eight) of these workers, the activities performed at hotspots are work-related. For the majority of these (thirty of thirty-four), their favorite hotspots (which are mostly libraries, bars, and restaurants) are even considered secondary workplaces.

As the sample size did not permit generalizing about WiFi users in Québec City, the research project chose to take advantage of the diversity present in the sample to identify different user types. A hierarchical cluster analysis was performed using variables related to technology and transport use, level of WiFi use at a hotspot (frequency and length) as well as whether or not work activities were performed at hotspots and the frequency of working at home. The cluster analysis $^{4}$ revealed three user profiles: Local Relaxers $(n=17)$, Urban Mobiles $(n=30)$ and Suburban Parents $(n=16)$. 
Table 11.1 outlines the three WiFi user profiles. The names given to each type are by no means applicable to each individual in the group, but attempt to summarize overarching trends. The Local Relaxers, for instance, include respondents for whom the proximity to their home is important when choosing a public or semi-public place offering free WiFi access. ${ }^{5}$ This group is made up of fewer university graduates (three of seventeen) in households mostly without children, with modest income (\$40,000-\$49,999/year on average). These individuals, who live and work predominantly near the Saint-Jean neighborhood close to the city center and in surrounding areas such as Limoilou and the post-war neighborhood of Sillery, ${ }^{6}$ walk often and occasionally travel by car. Local Relaxers do not conduct work-related activities as often as the other two groups and work rarely at home.

Urban Mobiles frequent WiFi hotspots to work or study. They are characterized by a nearly equal presence of male and females, whereas the other two types are predominantly male. Most of the respondents in this group travel daily either on foot or by public transport, which reflects the fact that among the fifteen individuals who listed their places of work and residence, most live along major public transport routes. About half of the members of this group are students while the other half are professionals with children, have a university diploma, and have a middle-range household salary $(\$ 60,000-\$ 75,000 /$ year on average). They conduct work-related activities at WiFi hotspots and work at home either between one to several times a day or one to several times per week.

The third user type, Suburban Parents, is characterized by a larger number of respondents in households with more than two children (ten of sixteen) who live mostly outside the city's public transport network ${ }^{7}$ in residential neighborhoods where local services are less proximate. Unsurprisingly, these individuals travel mostly by car. Of the half who list their place of work, all of them work near the Old City (where there is a concentration of public administrative offices) or the growing economic hub of Sainte-Foy. While the members of this group frequent WiFi hotspots close to their workplaces, this group is the only type of WiFi user identified that also uses WiFi during their children's activities, with several (five of sixteen) taking advantage of the WiFi offered at indoor ice rinks.

\section{Geographic disparities in WiFi use: a higher frequenting of hotspots in easily accessible mixed-use urban areas}

While the internet survey investigated the spatial and temporal practices of the three WiFi user types, it only hinted at the ways in which the overall network is used in relationship to the city. Mapping the server data provided by ZAP Québec revealed variations in WiFi use across the city. Figure 11.1 shows the distribution of the ZAP hotspots over the metropolitan area of Québec. The majority of hotspots are located near the city center on the north shore of the St. Lawrence River, while fewer are located on the south shore and in the suburbs. As previously mentioned, these places are mostly restaurants, libraries, bars, community centers, parks and public squares, and hockey arenas (Table 11.2). 


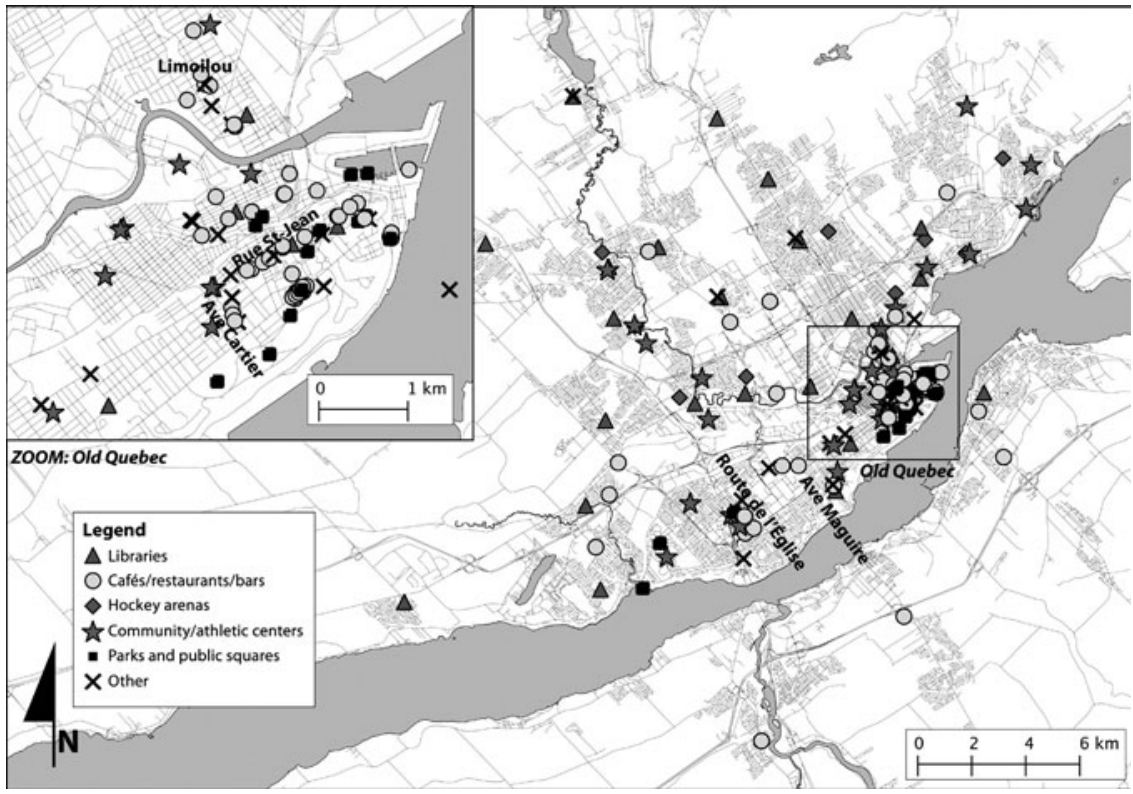

Figure II.I Distribution of ZAP Québec WiFi hotspots on the Québec City metropolitan area by type (@ M. R. Doyle).

Table II.2 ZAP Québec WiFi hotspots by type and status of inclusion in study as of September 2010

\begin{tabular}{lccc}
\hline Type & $\begin{array}{l}\text { Considered for the study } \\
\text { (February 2006-October 2009) }\end{array}$ & $\begin{array}{l}\text { Not } \\
\text { considered }^{\mathrm{a}}\end{array}$ & $\begin{array}{l}\text { Total (as of } \\
\text { September } \\
2010)\end{array}$ \\
\hline $\begin{array}{l}\text { Café/restaurant/bar } \\
\text { Community/athletic }\end{array}$ & 60 & 1 & 83 \\
$\quad$ center & 25 & 0 & 30 \\
$\begin{array}{l}\text { Library } \\
\text { Outdoor park and }\end{array}$ & 14 & 14 & 27 \\
$\quad$ public places & 5 & 0 & 19 \\
Hockey arena & 9 & 0 & 12 \\
$\begin{array}{l}\text { Tourism/hospitality } \\
\text { Schools }\end{array}$ & 4 & 0 & 11 \\
Theater and cinema & 6 & 0 & 9 \\
Other & 3 & 0 & 3 \\
Total & 8 & 0 & 17 \\
\hline
\end{tabular}

Note

a Hotspots that are not monitored and therefore were not included in the study, as no visitor count could be obtained. 
Other but less frequent types include hair salons, cinemas, and theaters, as well as tourist information offices.

Figure 11.2 shows the same distribution of ZAP hotspots, but this time adjusted to illustrate popularity. The relative size of the circle represents the average number of visits per day since the opening of the hotspot.

Mapping the server data reveals that the most frequented ZAP Québec hotspots are situated in the central neighborhoods of the city. Several highly frequented hotspots are also concentrated elsewhere, as can be better illustrated in Figure 11.3. These locations are typically commercial streets situated near residential neighborhoods. Overlaying the routes of city buses that pass by at least every fifteen minutes reveals that these hotspots are also situated near or along frequent bus routes. While the relationship is not necessarily causal, it is interesting to remark that the most frequented ZAP hotspots are located on commercial streets near residential neighborhoods and are easily accessible by public transport.

\section{Online in public: the pattern language of WiFi hotspots.}

As suggested by the results of the internet survey and the mapping of frequency, contextual elements, such as proximity to transport hubs or to the home and workplace, appear to play a role in the degree to which WiFi hotspots are

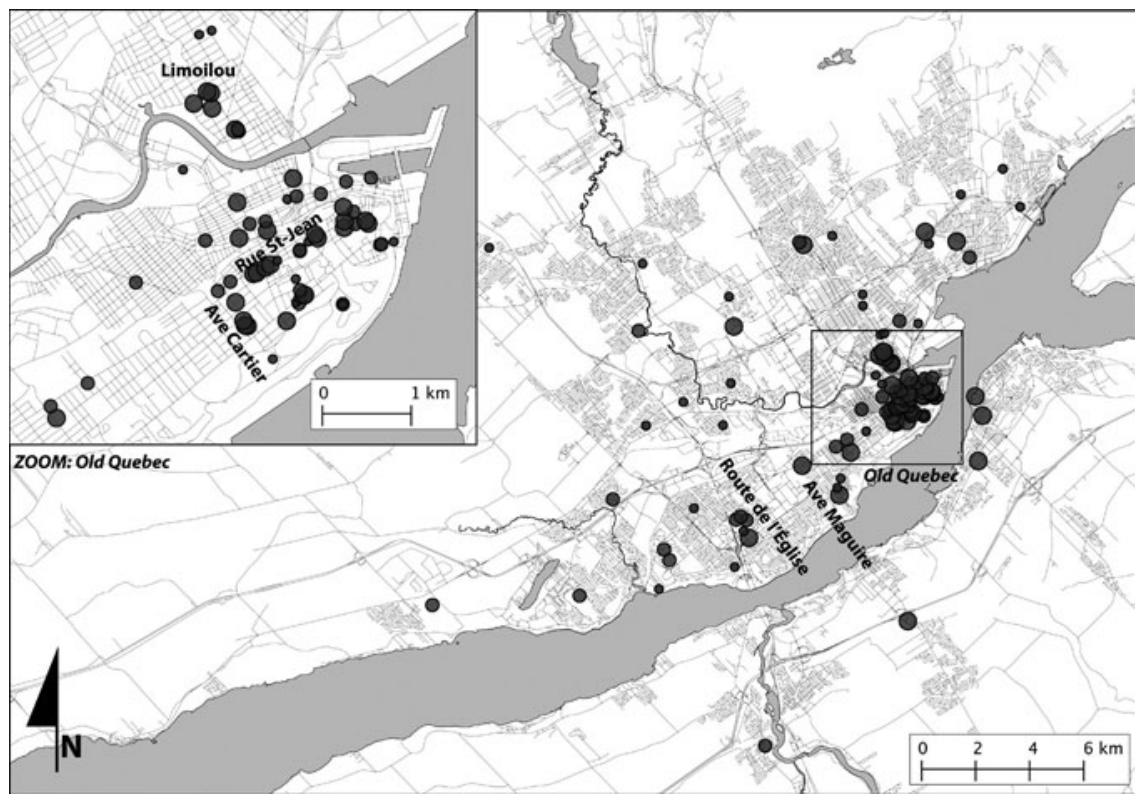

Figure II.2 Distribution of the most frequented ZAP hotspots (@) M. R. Doyle). 
frequented. The final portion of the study sought to verify these findings by performing a spatial analysis on a selection of nine hotspots, chosen by crossing the hotspots frequented by the three WiFi user profiles with the places that fell into the top quartile of the most frequented hotspots in the network. The final list of places (Table 11.3) was a selection of cafés, restaurants/bars, and libraries in six different neighborhoods.

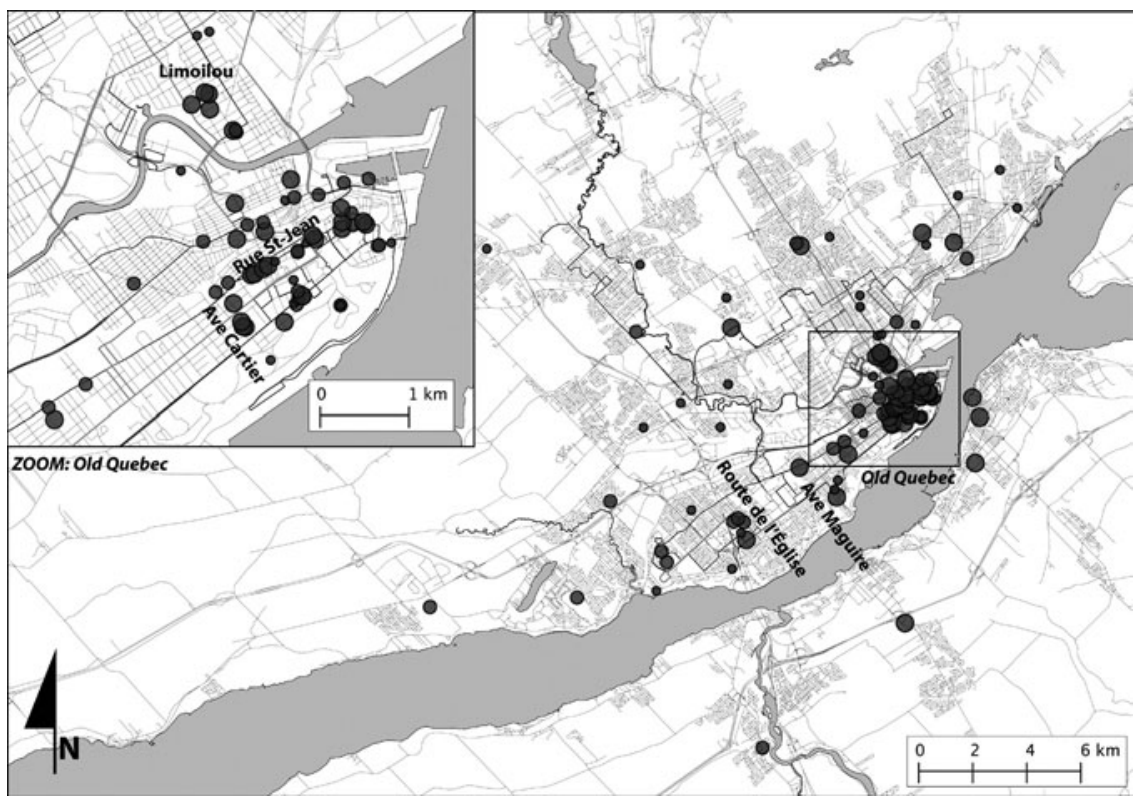

Figure II.3 Top 25 percent most frequented hotspots and public transport (green lines represent the Metrobus network serving high-demand axes every five minutes; blue lines represent standard bus routes that pass every fifteen minutes) (@ M. R. Doyle).

Table II.3 Hotspots visited as part of the spatial analysis

\begin{tabular}{lll}
\hline Name of hotspot & Type & Location \\
\hline Pub d'Orsay & Bar/restaurant & Old Quebec \\
Au Bonnet d'Âne & Bar/restaurant & Saint-Jean-Baptiste \\
Gabrielle-Roy Library & Library & Saint-Roch \\
Brûlerie Saint-Roch & Café & Saint-Roch \\
Pub Galway & Bar/restaurant & Montcalm \\
Le Bal du Lézard & Bar & Limoilou \\
La Fournée BIO & Café/bakery & Limoilou \\
Café Nagua & Café & Limoilou \\
Étienne-Parent Library & Library & Beauport \\
\hline
\end{tabular}




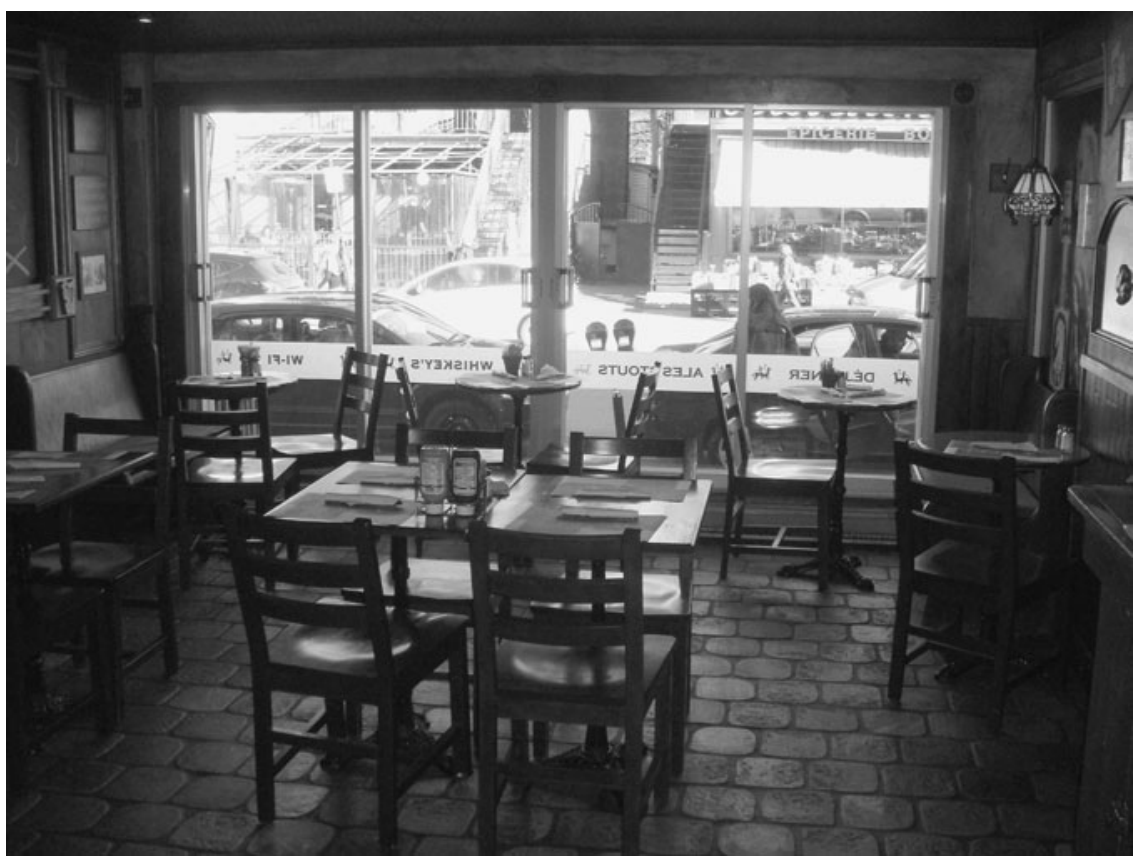

Figure I I.4 Pub Galway offers pub-goers a view of the activity on Avenue Cartier (@ M. R. Doyle).

This exercise sought to identify the sets of spatial configurations that were present across the most frequented and favorite hotspots. Analysis of each hotspot in plan (from above, cut at one meter) and in section (from the side, cut through the middle) allowed the list of patterns to be narrowed from an initial selection of forty-one to five: ACTIVITY POCKETS, WINDOWS OVERLOOKING LIFE, POSITIVE OUTDOOR SPACE, COMMON AREAS AT THE HEART, and MAIN ENTRANCE. What is interesting about the final patterns is that they all mediate the relationship of the hotspots to their context and, subsequently, the relationship of the WiFi users to nearby activities or spaces. For example, Pub Galway (Figure 11.4) is located along an urban commercial street on the Avenue Cartier (shown previously in Figure 11.3). Its generous view of the street from both the inside (the pattern WINDOWS OVERLOOKING LIFE) and from the outdoor terrace (POSITIVE OUTDOOR SPACE) offers WiFi users an opportunity to be spectators of a busy pedestrian area full of shops and restaurants (ACTIVITY POCKETS). Attention can also be drawn toward the inside of the space: At the Gabrielle-Roy Library, the worktables are arranged around a central atrium space where WiFi users have a view of the central circulation areas (COMMON AREAS AT THE HEART). The analysis also revealed that a highly frequented hotspot is one whose front door is 

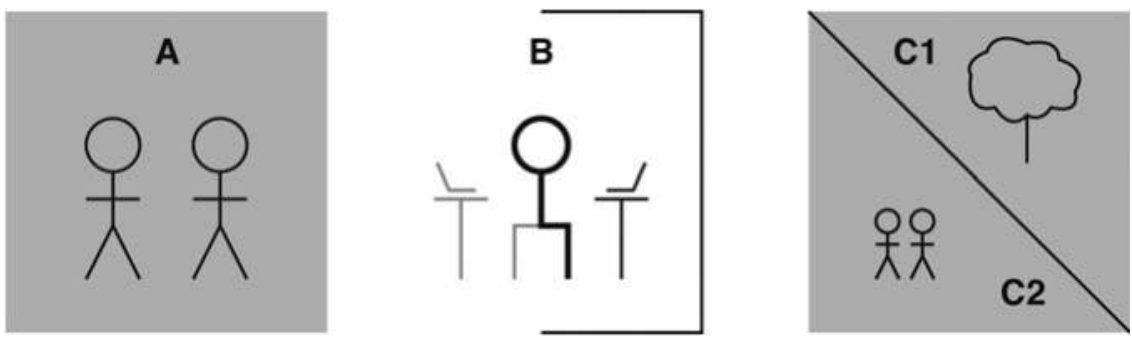

Figure II.5 Graphic representation of the WiFi user (A) with views of indoor (B) or outdoor (C) activities (@ M. R. Doyle).

highly visible from the street (MAIN ENTRANCE): six of the nine hotspots analyzed are, in fact, located on a street corner.

Figure 11.5 summarizes the spatial configurations of the most frequented hotspots in a simple diagram. Depending on the location of the WiFi user, the hotspot offers either a view of an indoor activity (A), or of a landscape (C1), or outdoor activity (C2). Although no indoor ice rinks were included in the spatial analysis, it is interesting to note that the ice rink follows a similar pattern language. The WiFi user is offered a view of the activity of the ice rink. As evidenced by the Suburban Parents WiFi user type, however, ice rinks are frequented for other reasons and wireless internet is used simply to spend time that might otherwise have been spent doing something else.

\section{Discussion: the relational programming of place}

The ability of architecture and urban planning to respond to mobile, fragmented activities depends upon an understanding of not only the impact of mobile technologies on individual people, but also on the places and the relationships between them that are restructured or reprogrammed. The empirical literature reviewed sheds light on the emerging spatial practices of individuals using ICTs in their professional lives, but the places themselves (the car, public transport, the home, or the office) lack contextual description. Where are these practices occurring and why? The WiFi user profiles observed in Québec City provide three examples of some ways in which these practices are spatially situated and suggest that spatial location may be as much enhancing and diversifying existing space-time geographies as creating new ones (Couclelis 2004). Local Relaxers tend to use WiFi close to home, Urban Mobiles look for places close to work or school and Suburban Parents use it in places they have to go for their children's activities.

If the investigation were to stop there, designing for mobile activities would address the needs of people carrying out ICT-supported activities in public spaces of their choice. Anthony Townsend and colleagues' (2011) Breakout! project attests to the challenge of developing design solutions for bringing a generic set of 
work-related activities into generally defined outdoor public places. Contrary to their expectation that the work place could abandon the office building completely, their collaborative design charrettes with mobile workers revealed the difficulty of defining the mobile worker as well as the amount of physical structure, in addition to power sources, signal strength, and connection speed, that is necessary in order for work to become mobile. The inability of the project to easily reconcile the relationship between the fixed and mobile workplace is actually more interesting than the initial hypothesis that the dissolution of one into the other was possible. WiFi users in Québec City return to the same hotspots because of their proximity to other places of interest. In this sense, work may break out of the office, but it is not entirely liberated from the structural logic of the city.

Analysis of the ZAP Québec network using data from 2006 to 2010 and the spatial and urban characteristics of the most frequented WiFi hotspots by the Québec City WiFi user population in 2010 suggests a complementarity between the frequency of use and the location of the hotspots in the city. Situating the hotspots in the broader urban context reveals that those frequented by Local Relaxers and Urban Mobiles are often located in or in proximity to highly trafficked areas. If in this case $\mathrm{WiFi}$ appears more to activate certain highly connected spaces, the hockey arenas rely more on their attractiveness as destinations for a popular activity (where WiFi is a bonus). Such latent syntactical logics are also important at the scale of the hotspot itself. The most frequented hotspots are not only easily accessible or home to popular pastimes; their spatial organization emphasizes the relationship of the individual to adjacent activities, whether indoors or outdoors. As such, it is interesting to know not only what motivates mobile workers to come to the office (Brown and O'Hara 2003) or to stay home (Hislop and Axtell 2009) and that ICTs can enable travel time to be valuable for both public transport users (Lyons et al. 2007) and automobile drivers (Laurier 2004), but it is also important to situate these places within urban contexts and sequences of travel.

Designing for mobile activities addresses first and foremost the urban scale, reading the underlying urban spatial logic and the common destinations or through-spaces of movement. The relationship between adjacent activity and accessibility suggests that places where people gather or converge would be prime locations for design interventions. Public squares and parks as well as places with a direct view of public transportation hubs or situated on commercial pedestrian streets with a view of street life seem to be where people desire WiFi the most. This is hardly surprising: Architects and urban planners have called intuitively for public places that allow people to be both spectators and performers of urban life since at least the 1980s (Bentley et al. 1985; Whyte 1980). Rather than counter this relationship, mobile technologies appear to reinforce it, making it possible to be spectators through physical presence and mediated communication (Gordon and de Souza e Silva 2011).

Archigram's 1964 Plug-in-City and Walking City projects imagined a world in which mobile building units or cities could detach from their current location to be reconnected and reconfigured spontaneously elsewhere (Sadler 2005). With 
the portability of contemporary mobile technology and the ubiquity of infrastructure allowing devices to communicate with dispersed forms of information and remote individuals, the reality today is that people are "plugging in" and moving from place to place (Abbas 2006), rather than the buildings themselves. Interaction designers are questioning the utility of designing only for the nomadic individual (Aurigi and De Cindio 2008): Location-aware mobile technologies are as much about contextually relevant information as they are about making the individual as context-independent as possible. Contextual particularities mean that the city is not an even playing field. In Québec City for example, most popular hotspots are located in highly connected areas, not off the beaten path. Even as WiFi is eclipsed by faster mobile internet speeds via $3 \mathrm{G}$ or $4 \mathrm{G}$, there is no reason to expect that one location will become more popular than another on internet access alone.

Perhaps urban planning and architecture need a new working metaphor that encompasses the emphasis on context called for by interaction designers. In Digital Culture in Architecture, Antoine Picon (2010) observes a transition from the production of autonomous objects (combustion engines, bicycles) to the production of semi-objects that depend upon existing infrastructure (computers with software, cell phones with towers) without which they would be meaningless. This creates a landscape upon which program (or activity) supersedes structure or form, recalling Malcolm McCullough's (2004) vision of an architecture (or urban planning) that focuses more on interconnections and experiences than formal objects. Connections are not merely within the building or project (as a closed autonomous system) but open to the entire urban landscape. Current advances in office design illustrate the challenges and limits of neglecting connections at the urban scale. Many office projects tend to internally reproduce the elements of the city (streets, cafés, public open spaces), rather than allow workrelated activities to spread outward into the city (Duffy 2008). The challenge is one of programming at both the architectural and urban scales. A new metaphor overlaps both scales: people plug in to places that plug in to programmatic landscapes.

Designing places for mobile activities merges macro-level investigation of the relationships between urban places and consultations with local citizens. The detailed methods of architectural programming, from prolonged observation to participatory design exercises could be brought to an urban scale, revealing the immaterial realities that might orient the architectural or urban project and better connect it to the material or immaterial networks that traverse the project site and its surroundings (see Ole B. Jensen's contribution to this volume for specific examples of what could comprise the designer's ICT “toolbox", Chapter 13). This kind of evidence-based design would be interested not only in the practices of the future users of the project, but also those in the surrounding area. The results of the study presented here suggest that future implementation of WiFi in Québec City could involve ZAP Québec, the provider, as well as decision makers and citizens. Its objective would be to 
seek solutions for the material realities of specific well-connected urban places. Contrary to the Breakout! project, work would be only one of several activities "breaking out" (with others perhaps "breaking in") and the participatory exercises could unearth the synergies between the different types of activities that people want to most engage in, with or without mobile technologies. This is one form of relational programming: a design and planning effort that connects the material and immaterial, the mobile and the fixed. The mobile (re)activates the local.

\section{Conclusion}

Departing from the idea that places are constituted of various forms of mobility and fixity, this chapter began by reviewing recent empirical studies on the complementarity between travel and ICT use and theoretical writings from architecture and interaction design on the relationship between mobile technology and context. Although the theoretical literature called for an increased consideration of context in place-making, the empirical studies focused more on the mobility of individuals than on the places where mobile technology use was occurring. In order to investigate the spatial nature of ICT use, this chapter referred to findings from a study of WiFi hotspots and users conducted in Québec City from 2008 to 2010. In addition to corroborating some of the evidence found in larger quantitative studies, it revealed the heterogeneity of WiFi use and identified three user profiles differing according to their spatial and temporal ICT practices. Analysis of the use of all hotspots since early 2006 revealed that the most popular places are located near commercial streets and transportation hubs mostly in mixed-use neighborhoods. Further investigation of the top quartile of these places revealed that they share many of the common elements of successful public places: They offer the WiFi user a seat at the spectacle of daily life. The discussion questioned existing metaphors for imagining the impact of mobile technologies on the fixed places of the city, proposing an alternative metaphor by which buildings achieve functional meaning through their interconnections with neighboring activities at multiple scales. Relational programming calls for the exploration of different forms of movement in creating quality places where mobile people come to rest.

\section{Acknowledgments}

The author would like to thank ZAP Québec in Québec City for their precious collaboration, his research advisor, Carole Després, and colleagues at the Interdisciplinary Research Group on Suburbs (GIRBa) at Université Laval. A special thanks goes also to the editors for their constructive comments on earlier versions of this chapter. 


\section{Notes}

1 While these studies all looked at ICT use in travel, ICT can also play an important role in chosen or imposed forms of immobility (see, for example, Kesselring 2006).

2 ZAP (Zone d'accès public or "public access zone").

3 For a more detailed explanation of the methodology, please see Doyle 2011.

4 This type of cluster analysis is appropriate for small samples and was used for exploratory purposes and then followed up with a k-cluster analysis, revealing the robustness of the hierarchical cluster analysis (few cases changed groups). This process was adopted from Lenz and Nobis (2007).

5 All of the respondents had internet access at home.

6 Twelve of the seventeen respondents listed the postal codes of their places of residence and work.

7 Eight of the sixteen respondents listed the postal codes of their places of residence and work.

\section{References}

Abbas, Y. (2006) Neo-nomads: Designing environments for living in the age of mental, physical and digital mobilities, Doctorate of Design thesis, Harvard University, Cambridge, MA.

Afanasyev, M., T. Chen, G. M. Voelker, and A. C. Snoeren (2008) Analysis of a mixeduse urban WiFi network: When metropolitan becomes Neapolitan, Proceedings from IMC '08: Proceedings of the 8th ACM SIGCOMM Conference on Internet Measurement, New York, NY.

Alexander, C., S. Ishikawa, and M. Silverstein (1977) A Pattern Language: Towns, Buildings, Construction, Oxford: Oxford University Press.

Amin, A. and N. Thrift (2002) Cities: Reimagining the Urban, Malden, MA: Blackwell Publishers Limited.

Aurigi, A. and F. De Cindio (2008) Augmented Urban Spaces: Articulating the Physical and Electronic City, Aldershot: Ashgate.

Axtell, C., D. Hislop, and S. Whittaker (2008) Mobile technologies in mobile spaces: Findings from the context of train travel, International Journal of Human-Computer Studies, 66(12): 902-915.

Benkler, Y. (2006) The Wealth of Networks: How Social Production Transforms Markets and Freedom, New Haven, CT: Yale University Press.

Bentley, I., A. Alcock, P. Murrain, S. McGlynn, and G. Smith (1985) Responsive Environments: A Manual for Designers, London: Architectural Press.

Brown, B. and O'Hara, K. (2003) Place as a practical concern of mobile workers, Environment \& Planning A, 35(9): 1565-1587.

Castells, M. (2000) The Rise of the Network Society: The Information Age: Economy, Society and Culture vol. 1 (second edition), Malden, MA: Blackwell Publishing.

CEFRIO, Léger Marketing (2008) Netendances 2007: Utilisation d'internet au Québec (version abrégée), Montréal: CEFRIO.

Couclelis, H. (2004) Pizza over the Internet: E-commerce, the fragmentation of activity and the tyranny of the region, Entrepreneurship and Regional Development, 16(1): 41-54.

Doyle, M. R. (2011) Designing for mobile activities: WiFi hotspots and users in Quebec City, master's thesis, Université Laval, Québec.

Duffy, F. (2008) Work and the City, London: Black Dog. 
Élections Québec (2008) Internet sans fil. Un nouvel enjeu electoral. Élections Québec, available at: http://elections.radio-canada.ca/elections/quebec2008/2008/11/14/ 004-ADQPLQ_Internet-s-f_QC.shtml.

Forlano, L. (2008) When code meets place: Collaboration and innovation at WiFi hotspots, Doctor of Philosophy thesis, Columbia University, New York.

Gordon, E. and A. de Souza e Silva (2011) Net Locality: Why Location Matters in a Networked World, Malden, MA: Wiley-Blackwell.

Graham, S. (1998) The end of geography or the explosion of place? Conceptualizing space, place and information technology, Progress in Human Geography, 22(2): $165-185$.

Graham, S. and S. Marvin (1996) Telecommunications and the City: Electronic Spaces, Urban Places, New York, NY: Routledge.

Gupta, N. (2004) Grande Wi-Fi: Understanding what Wi-Fi users are doing in coffeeshops, Master of Science thesis, Massachusetts Institute of Technology, Cambridge, MA.

Hampton, K., L. Sessions, E. J. Her, and L. Rainie (2009) Social Isolation and New Media: How the Internet and Mobile Phones Impact Americans' Social Networks, Washington, DC: Pew Internet \& American Life Project, available at: www.pewinternet.org/files/old-media/Files/Reports/2009/PIP_Tech_and_Social_Isolation.pdf.

Hislop, D. and C. Axtell (2009) To infinity and beyond: Workspace and the multilocation, New Technology, Work and Employment, 24(1): 60-75.

Jensen, O. B. (2013) Designing mobilities - staging materialities of mobilities, paper presented at the Danish Sociology Congress "Mobilitet og by," January 24-25, Roskilde University.

Kesselring, S. (2006) Pioneering mobilities: New patterns of movement and motility in a mobile world, Environment and Planning A, 38: 269-279.

Laurier, E. (2004) Doing office work on the motorway, Theory, Culture \& Society, 21(4/5): 261-277.

Lee-Gosselin, M. and L. F. Miranda-Moreno (2009) What is different about urban activities of those with access to ICTs? Some early evidence from Québec, Canada, Journal of Transport Geography, 17(2): 104-114.

Lenz, B. and C. Nobis (2007) The changing allocation of activities in space and time by the use of ICT - "fragmentation" as a new concept and empirical results, Transportation Research Part A: Policy and Practice, 41(2): 190-204.

Licoppe, C., D. Diminescu, Z. Smoreda, and C. Ziemlicki (2008) Using mobile phone geolocalisation for "socio-geographical" analysis of co-ordination, urban mobilities, and social integration patterns, Tijdschrift voor Economische en Sociale Geografie, 99(5): 584-601.

Lyons, G., J. Jain, and D. Holley (2007) The use of travel time by rail passengers in Great Britain, Transportation Research Part A, 41(1): 107-120.

Mackenzie, A. (2010) Wirelessness Radical Empiricism in Network Cultures, Cambridge, MA: MIT Press.

McCullough, M. (2004) Digital Ground: Architecture, Pervasive Computing, and Environmental Knowing, Cambridge, MA: MIT Press.

Meyrowitz, J. (1985) No Sense of Place: The Impact of Electronic Media on Social Behavior, New York, NY: Oxford University Press.

Mitchell, W. J. (2003) Me++: The Cyborg Self and the Networked City, Cambridge, MA: MIT Press. 
Mokhtarian, P. L. (2002) Telecommunications and travel: The case for complementarity, Journal of Industrial Ecology, 6(2): 43-57.

Nicolaou, L. (2006) Emerging building forms and accommodation solutions: New building typologies or distinctive place-making, in J. Worthington (ed.), Reinventing the Workplace (second edition), Burlington, VT: Architectural Press.

Oldenburg, R. (1989) The Great Good Place: Cafés, Coffee Shops, Community Centers, Beauty Parlors, General Stores, Bars, Hangouts, and How They Get You Through the Day, New York, NY: Paragon House.

Picon, A. (2010) Culture numérique et architecture—Une Introduction, Basel: Birkhäuser.

Putnam, R. (2000) Bowling Alone: The Collapse and Revival of American Community. New York, NY: Simon \& Schuster.

Sadler, S. (2005) Archigram: Architecture Without Architecture, Cambridge, MA: MIT Press.

Schwanen, T. and M.-P. Kwan (2008) The internet, mobile phone and space-time constraints, Geoforum, 39(3): 1362-1377.

Sennett, R. (2005) Capitalism and the city, in S. Read, J. Rosemann, and J. V. Eldijk (eds.), Future City, New York, NY: Spon Press, pp. 114-124.

Sheller, M. and J. Urry (2003) Mobile transformations of "public" and "private" life, Theory, Culture \& Society, 20(3): 107-125.

Sheller, M. and J. Urry (2006) The new mobilities paradigm, Environment and Planning A, 38(2): 207-226.

Srinivasan, K. K. and P. N. Raghavender (2006) Impact of mobile phones on travel: Empirical analysis of activity chaining, ridesharing and virtual shopping, Transportation Research Record: Journal of the Transportation Research Board, 1977: 258-267.

Therrien, Y. (2009) Subvention de 200,000 \$ pour ZAP Québec, Le Soleil, available at: www.cyberpresse.ca/le-soleil/actualites/science-et-technologie/200902/19/01-829203subvention-de-200-000-pour-zap-quebec.php.

Thulin, E. and B. Vilhelmson (2007) Mobiles everywhere: Youth, the mobile phone, and changes in everyday practice, Young: Nordic Journal of Youth Research, 15(3): 235-253.

Townsend, A., A. Simeti, D. Spiegel, L. Forlano, and T. Bacigalupo (2011) Breakout!: Escape from the office, in M. Shepard (ed.), Sentient City: Ubiquitous Computing, Architecture, and the Future of Urban Space, Cambridge, MA: MIT Press, pp. 128-151.

Wellman, B. (2002) Little boxes, glocalization, and networked individualism, in M. Tanabe, P. van den Besselaar and T. Ishida (eds.), Digital Cities II: Computational and Sociological Approaches, Berlin/Heidelberg: Springer Verlag, pp. 10-25.

Whyte, W. H. (1980) The Social Life of Small Urban Spaces, Washington, DC: Conservation Foundation. 


\section{Chapter 12}

\section{The power of place and perspective}

\section{Sensory media and situated simulations in urban design}

\section{Gunnar Liestøl and Andrew Morrison}

It may be said that a Copernican turn in media is underway. Increasingly, we are set free from the physical constraints of traditional media locations for our regular information feed, be it the cinema house, the TV set, the radio, or our favorite reading chair. Instead, information in its many forms and the interfaces we activate to access, produce, and share it, gravitate around us as individuals. They follow us everywhere, and always, and, importantly, they do so as we move. This gives new meaning to the significance of location and perspective. Added to these dimensions is the further condition that dynamic information media engage us with the sensorial in the situations of their use. While earlier media were conceived as sensorial extensions of our nervous system (as championed by McLuhan 1964), now the metaphor applies to the handheld media devices themselves. These terminals are no longer only subordinate augmentations of our human sensory system, but have their own complex sensorial capabilities as well. We may say, then, that we are experiencing the age of situated and sensory media.

This turn away from the physical restrictedness and immobility of traditional media hardware to the individual body of the users and their always-on mobile devices will most probably cause changes in the power of place and perspective (Bratton 2009). In the following pages we explore these consequences in the context of an urban design case from Scandinavia that concerns the placement and selection of a new national museum within wider processes of urban change. The addition of location-based technologies and dynamic situated media (that are processed and interpreted on site) raises a number of challenges in negotiating relations between place, position, perspective, and perception. The use of these new technologies and related negotiations around them may open up issues for cultural policy and decision making. Consequently, they may also have some bearing on our understanding of sites of public cultural communication.

Below, we first describe the basic features of the digital platform we have been experimenting with over the last several years: We have called this situated simulations (sitsim), and it has been identified as a form of indirect augmented reality (Wither et al. 2011). Alongside this is the potential inclusion of yet another mode of visual, spatial, and now dynamic situated communication as 
part of urban planning and change (Schnädelbach 2009). Digital platforms like sitsims are becoming part of the tools we employ to imagine our cities culturally and technically (Donald 1999; Williams and Dourish 2006; Farman 2012). Location-based technologies provide us with additional technical and spatial affordances for positioning designs for the built environment in situ (e.g., Felix et al. 2008). They also open out for further extensions of our projections and perceptions of the multimodally mediated designs of architects, cultural institutions, and planning agencies. This is already apparent, for example, in debates surrounding developments on delicate cultural and political sites such as the World Trade Center in New York. It reaches into the online mediation of leading architectural museum complexes such as the Tate Modern in London and the new National Museum of 21st Century Arts (MAXXI) in Rome (Pierroux and Skjulstad 2011). Architectural competition finalists are also often featured in research and design publications as exemplars of emergent and contemporary innovation in materials, computing, form, and aesthetics. Competitions for public buildings, especially large cultural institutions such as national museums, are filled with political contest; they are sites of significant mediation as part of highly competitive processes of selection and award. National museums often become cultural landmarks that are architectural as much as they are known by their collections, curatorial prowess, and quality of exhibitions. These architectural competitions are foregrounded in complex processes that entail the projected cultural location of prestige projects that will eventually be lodged in the built environment.

We draw on a developmental approach to research by design, which involves making and analyzing over time and with reference to emerging mobile technologies. In contrast to social science mobilities research (e.g., Büscher et al. 2011; Hjorth et al. 2012) influenced by sociology and studies of technological systems, our inquiries and productions are also closely related to practices and published research in the digital humanities (Morrison and Mainsah 2012). We draw on these as resources in designing and communicating how the sitsim redefines mediation of place and perspective. Next, we present the core case in which we have applied sitsim with respect to the planned building of the new national museum in downtown Oslo. Here we particularly focus on the public visualization of the new architecture and briefly contextualize it in terms of cultural debates, policies, and decision making. We then move on to describing the features of the sitsim and its design and trial evaluation with international students on location. This leads to a discussion of the results in context of place and perspective. Finally, we place the findings in a wider frame of interaction design, mediated communication, and cultural discourse and suggest further experiments and scenarios for this kind of experimental research and development.

\section{Augmented realities: past and future}

Since the virtual reality hype collapsed in the mid-1990s the field of augmented reality has proved itself to be an experimental research tradition in steady 
growth. Augmented reality has matured and become a more diverse platform expanding and moving beyond its mixed reality origin as described by Milgram and Kishino (1994). The recent emergence and availability of sensor-based and location-aware smartphones and tablets challenges the original taxonomy of augmented reality and how it has subsequently been characterized in the research literature (Azuma 1997; Azuma et al. 2001). With mobile sensory devices, the mixed reality boundary no longer resides at the level of the display. The frame of the display has itself become the border between the computer graphics generated environment and the real (Liestøl 2011). These new configurations, based on the sensor fusion approach as opposed to fiducial markers and pattern recognition, have been named indirect augmented reality (Wither et al. 2011). A situated simulation is an example of this indirect kind of mobile augmented reality.

In a situated simulation there is approximate congruity between the user's visual perception of the real physical environment and the user's visual perspective into a 3D computer graphics environment as it is represented on the full screen of the device. The relative correspondence between the real and the computer-generated perspectives is obtained by letting the artificial camera's position and movement in the $3 \mathrm{D}$ environment be controlled by the location, movement, and orientation input from the hardware sensors (GPS, accelerometer, gyroscope, and magnetometer). As the user moves both herself and the device in real space, the perspective inside the computer graphics space changes accordingly. This form of constructed representation is then applied to simulate alternative versions of a given location in situ. Such a simulation may relate to and display information and versions of the environment which are no longer in existence, hidden, or have not yet come into existence; that is past, present, or future dimensions and topics, or even completely fictional spaces and scenarios.

So far we have primarily concerned ourselves with simulations of past topics: a reconstruction of the original Mission Dolores in San Francisco from 1791; a burial scene of the Oseberg Viking ship and a Viking settlement in Norway from the early Viking Age; the Parthenon and Erechtheion temples on the Acropolis in Athens; the republican and imperial fora in ancient Rome; as well as a reconstruction of the fortified city of Phalasarna on Crete as of 335 BC. Feedback from continual and controlled user testing during the design of these sitsims has shown that users appreciate the incremental value of the simulated environments in that they augment the experience of the specific place in engaging and rewarding ways (Liestøl et al. 2011, 2012; Liestøl and Morrison 2013).

Research and development of the sitsim platform can be exemplified by our efforts to create simulations of the Roman Forum. This has been an experimental process of trial-and-error involving students from both the University of Oslo and the Norwegian Institute in Rome. We have designed and tested numerous prototypes on location. The sitsim was originally a rudimentary reconstruction of Julius Caesar's Temple in the Forum around its completion in $29 \mathrm{BC}$. It has evolved into a more complex environment involving the whole Forum with reconstructions from both $44 \mathrm{BC}$ and $29 \mathrm{BC}$ including sequences of actions and 
events related to Caesar's funeral and cremation. These versions have also served as narrative investigations, experimenting with flash backs as well as flash forwards by means of parallel movements in both time and space (Liestøl 2011). ${ }^{1}$ Results from repeated on-location testing with students and school children across all our productions clearly indicate that this is a viable platform for further development and may be shared to form potential genres suitable for various user contexts from research and education to informal learning and guiding. Users have found it particularly rewarding to experience the double perspective the sitsim renders possible and the added value the extra perspective creates in its interaction with the real place.

In developing these sitsims, we were mindful that mobile augmented reality has its precedents in print media. As visitors to Rome we are offered books showing combined views of new and old. A common rendering of these print display techniques presents a page with a current photographic image on top of which you may turn a partly transparent leaf combining the photographed remains of ancient buildings with reconstructions of the absent parts. Turning the transparent page back and forth gives us the opportunity to compare the past and the present over time. Other types display the past and present concurrently with images positioned on opposing pages in an open book. Most common examples are the Then and Now books with a display tradition optimized in the eminent work of Mark Klett (2006). In the first version the juxtaposition is

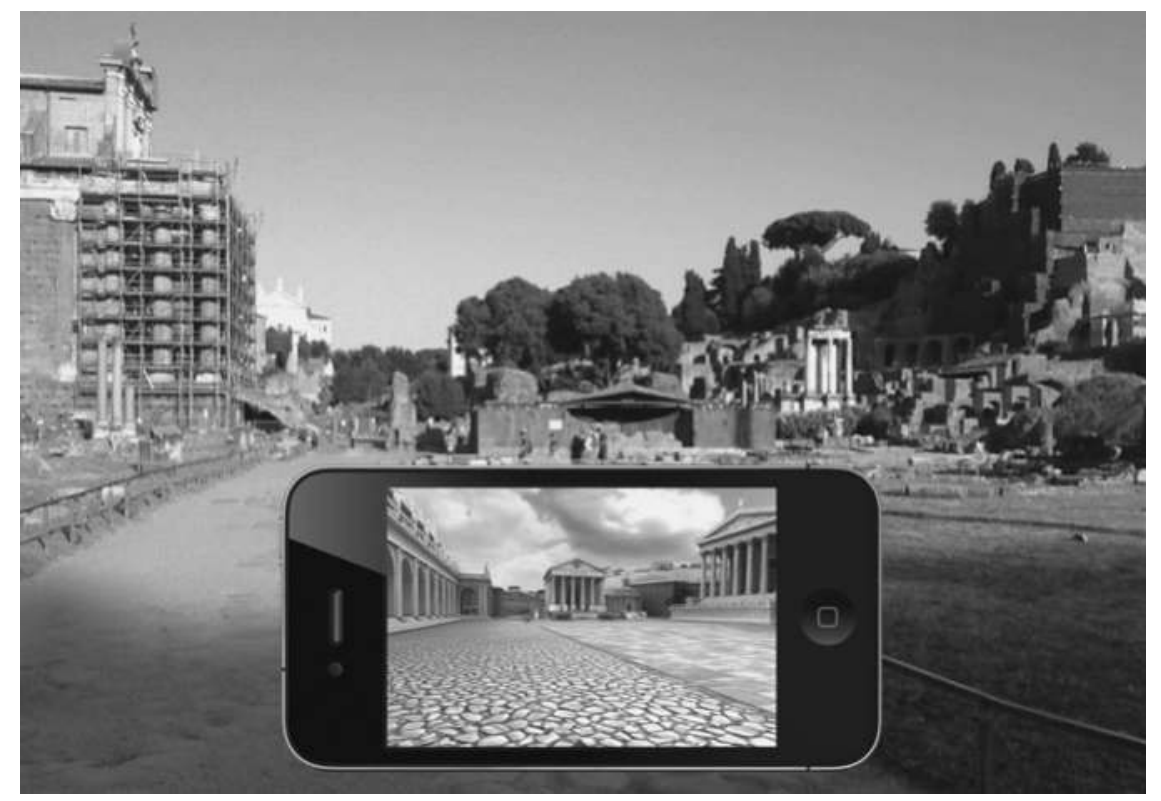

Figure 12.I The Roman Forum sitsim showing a now-and-then snapshot of the Via Sacra looking toward the Temple of Caesar (@ G. Liestøl). 
temporal, in the latter it is spatial. These different modes demand and produce different forms of perspectival actions from the user, turning pages as opposed to switching gaze. The temporal perspective is more sequential and memory demanding, while the spatial perspective gives room to simultaneous comparison of pictorial elements. This difference of display techniques in print media is mirrored in mobile augmented reality. The mixed reality (combined screen) solution gives a layered palimpsest image where the difference between past and present is turned on and off, while the indirect augmented reality mode is similar to the spatial juxtaposition of the real and the computer-generated perspectives in the now-and-then display tradition.

Based on these experiences (Liestøl et al. 2011) we have more recently experimented with future topics, constructing a simulation of a planned building project in downtown Oslo, Norway. Using mobile augmented reality for preconstructions of future buildings and structures also has its precedents in print technology as well as physical 3D models.

Architectural model making has undergone considerable transformation in the past two decades, principally through the near encompassing use of computeraided design/computer-aided manufacturing (CAD/CAM) technology and tools. All in all, models have a double representational role, as we have argued earlier in respect to developments in digital media (Liestøl et al. 2003). On the one hand they are scaled renderings of larger projected buildings. On the other hand, they become communicative artifacts in their own right and mediate cultural values and projected and intended outcomes. In the selection of media in architectural model making Nick Dunn (2010) offers the student options that range from paper and cardboard; wood; Styrofoam and plastic; resin, clay, and cast materials; steel and other metal works; CAD/CAM; and photography and film. Edward Robbins (1994) has traced, for example, the significance of drawing in the work of a range of leading architects; the views of Kevin Lynch (1960) have continued to be influential in visualizing urban contexts where overlays, annotations, and projections have now been extended into virtual spaces (e.g., Schnädelbach 2009).

We have also witnessed a turn toward the digital in architectural and urban design (McCullough 1998, 2004), with the parametric in architectural design, such as shown in the Verb book series by Actar (e.g., Meredith et al. 2008), influencing too the types of public buildings and mediations we meet as consumers and future dwellers. Parametric design refers to the possible fluid relationships between componential objects in unfolding design processes and systems, maintaining aspects while modifying others through transformations and iterations. While the tools that enable such dynamics in design and the resulting aesthetics of built designs might be infused with the marks of such tools, we see their effect across studios, locations, and competitions. Today architectural competitions migrate these tools and techniques from their own "drawings" in CAD suites to companies that then render these as filmic materializations (see Hah et al. 2008) that have persuasive and rhetorical force, as has been the case across 
media (Tostrup 2010). Importantly, architectural competitions come to be an enduring location for public use, one that is now interlinked with locative media technologies from Quick Response (QR) codes, Radio Frequency Identification (RFID) tags and social media messaging. Mobile devices already enable the download and generative use of applications ("apps") for overlaying information and interpretations upon screened content and actual sites of interest in a mode of "net locality" (Gordon and de Souza e Silva 2011).

Clearly, digital tools have a bearing on the workflows and activities as well as the practices within which a mix of professionals are now connected, from government planners to urban developers, through cultural policy makers to museum curators. Architectural competitions are characterized by intricate processes of planning, promotion, tenders, and selections (Chupin 2011), much of which is outside the purview of the wider public. However, appearance of completed designs and designs - whether by select experts or wider committee - has begun to change rather dramatically with the advent of social media and locative annotation especially as the final, mediated public stages of such contests increasingly come under public scrutiny. We have already seen several highly contested competitions that have shifted beyond their formal professional boundaries into debates across media types, interest groups, and earlier linear processes of formal decision making. Today, architectural competitions for large cultural centers and their relationships to changing urban landscapes and lifeworlds are very much in the public eye and claim in the Nordic context to include a measure of dialogue, albeit in terms of management rather than public hearings (Kreiner et al. 2011; Rönn 2011). Planning and design specialists to some degree are now forced to meet the responses of a varied public as projected buildings and their demonstrated uses are reviewed and discussed. These processes increasingly take place online and via social media.

However, it is still questionable as to what effect these wranglings and contested interpretations have in wider overall planning processes: Large public buildings, as well as private cultural institutions, are tendered on the basis of massive and often intricate funding mechanisms and alliances. The public meets the tail end of a large process, rarely having enough of a powerful flick to return it to its major redesign or inception. However, tenders and competition submissions now entail a mix of media: rarely do we see paper-based models, but in their place filmic renderings of the to-be-built. This is typically a world that may be described as "unreal estate" (Morrison and Skjulstad 2010) by way of its use of projected futures and hyper-real aesthetics that are enacted as persuasive devices in promoting preferable, beguiling, and better "realized" futures. Just as architectural competition now involves a high degree of film as its main mediational means, locative media technologies that draw on a mix of place, navigational, and situated mediation have begun to be taken up as part of the repertoire of architects' offices. Here the tablet has featured prominently as architects have experimented with its applications, such as Layar, and in layers of "augmented" mediation of urban augmented reality (UAR) app of the Netherlands Architecture 
Institute (Verhoeff 2012: 129) that can be seen as a performative cartography enabled by the smartphone and its capacities to facilitate our production of hybrid screenscapes (ibid: 119). It is such dynamic qualities that we wanted to embody in our own applied uses of indirect augmented reality in the context of the new national museum in Oslo.

\section{Forum artis-the New National Museum}

Planning and erecting public buildings in Oslo is not an easy matter, particularly when cultural institutions are involved. The ongoing and now protracted political conflicts concerning a new Edvard Munch Museum is only one of many examples of urban planning and change, which continue to haunt the Norwegian public sphere and paralyze political decision making. The history of the new national museum is also typical of this tendency. The planning process for building on the current site (Vestbanen) has been going on for decades and several competitions have been held. A winner (OMA/SpaceGroup) was declared after an international competition in 2002. But a new competition was announced in 2009. After a second round the contribution "forum artis" by Kleihues + Schuwerk was declared the winner from among five other final contenders. Their ambition is that the new museum will be "a vital national research and resource center for the visual arts. The museum complex will distinguish the National Museum as an international exhibition and communication arena" (official leaflet). The Norwegian parliament approved the project in 2013, and the National Museum is planned to be completed by 2019.

The visualizations of the Kleihues + Schuwerk architecture have been published in various media and are available both on the web and in print. ${ }^{2}$ The illustrations included in these presentations follow a clear pattern. The aim to exhibit the yet-to-be-built structure in easy to understand overviews is obvious both with still image renderings in print and on web pages, as well as in video sequences. The relationship between framing and position tends to be of two kinds: (1) either the perspective is a bird's-eye overview with a distant wide angle framing, including as much as possible of the contextual environment (see Figure 12.2), or (2) the position and perspective is on the ground as a street level view and the structures/building elements are close up, with a focus on detail, not overview (see Figure 12.3). The combination of street-level perspective and (distance) overview framing, however, cannot be found in the various illustrations used to present the building complex.

\section{Forum artis as a situated simulation}

Contact and collaboration with the research and development department at the Norwegian Public Construction and Property Management (Statsbygg) led to the sitsim of the planned new national museum. Statsbygg is responsible for largescale national building projects in Norway. Their motivation for embarking on 


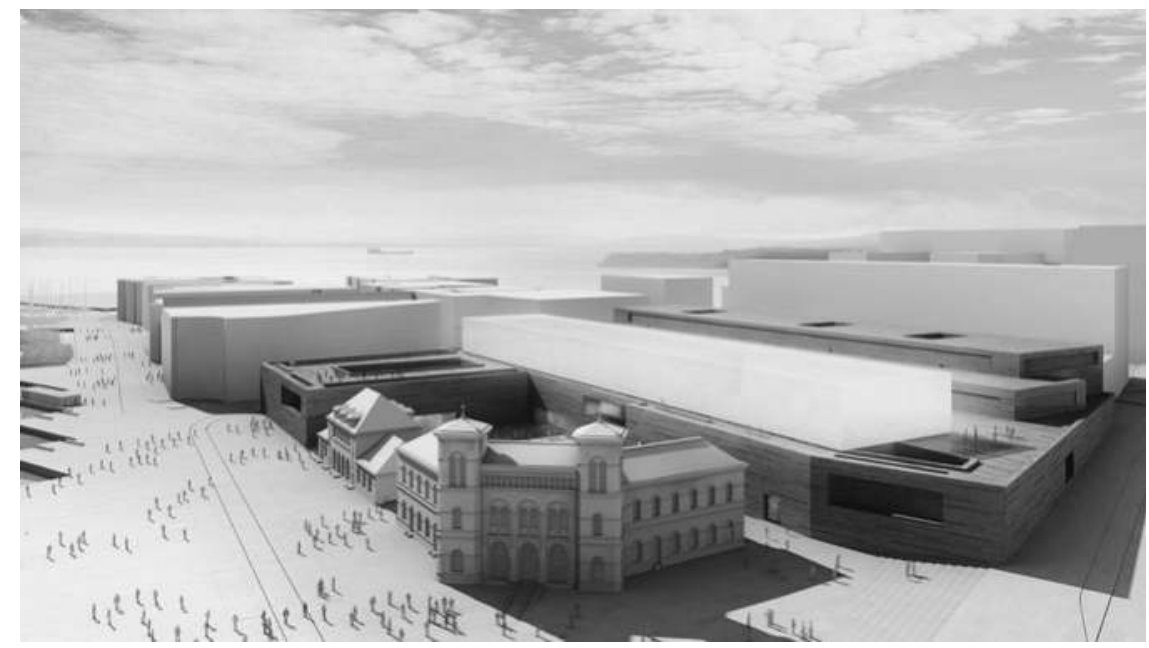

Figure 12.2 Example of perspective combining high altitude bird's-eye overview and distance (๑) Statsbygg).

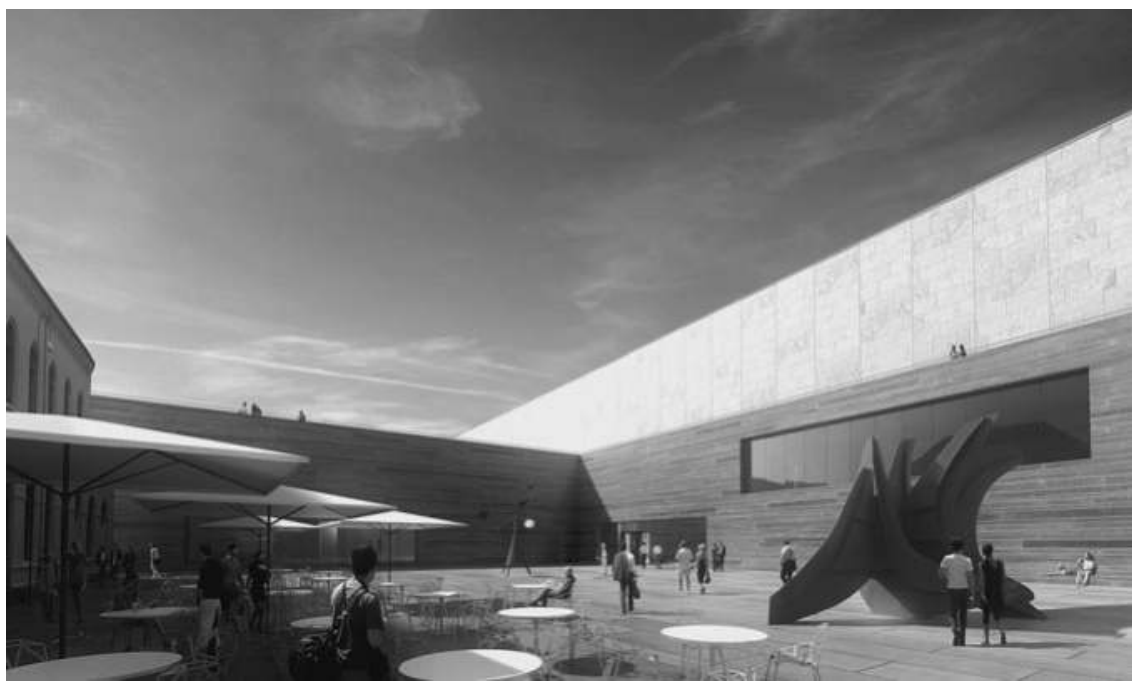

Figure 12.3 Example of perspective combining street level, close up and detail. Perspectives combining large frame, wide angle, and distance are absent from the presentations (@ Statsbygg). 
this particular sitsim project was two-fold: They wanted to explore new channels and options for dissemination of planned and ongoing projects to the public, and they intended to explore the possible interface between their industry/domain standard, the BIM-platform (Building Information Modeling) - and the basic tools applied for the sitsim platform (Unity3D with export to both iOS and Android). The simulation itself was then designed to be demonstrated and tested by representatives from Statsbygg's research and development section as well as representatives from The New National Museum Project. Production of the prototype (NasMus for short) was carried out in the fall of 2011 and demonstrated and tested on location that November. The demo of the prototype was by all accounts a success and consequently led to additional trials over the subsequent months. One of the reactions we noticed during these trials was the fact that the people close to the planning process found the relative size between the old and the new building to differ from what they expected. To better understand this response we decided to have a more systematic user test and evaluation. We return to this in the next section.

Based on 3D models and other documentation and material from the planning process, a rudimentary environment was created including the new museumforum artis - the old railroad station, some of the adjacent structures, and parts of the harbor. The sitsim environment had only two positioned links: One included links to Statsbygg's website for the project and the other had a "fly-in" function to access an imagined exhibition room of the Edvard Munch collection on one of the upper floors. In this case, the artificial camera is lifted vertically and fixed in a central position inside the gallery room. The ability to tilt and pan the camera, however, is intact. Consequently, the user can orient and move the artificial camera inside the graphically reconstructed gallery in any direction or angle while its position remains stable. Two universal links (buttons) were included in the hide/show dock at the bottom of the screen. One of these was for making the link layer invisible (turning informational mode off) and one for changing and adjusting the altitude of the artificial camera to twelve meters $( \pm 50$ percent).

The adjacent and peripheral buildings were given a simplistic representation in a light gray color. For the Oslo West Station we used photography-based textures without editing or retouching the images themselves. The new museum building was textured with available resources provided by Statsbygg. For the demos we primarily used the iPad2, but also the iPhone 4 . We also activated the built-in feature "Tilt offset" which causes the artificial camera to be lifted about fifteen degrees relative to vertical tilt of the device. The reason for this feature is to not have the screen of the device in the middle of the user's sight and thus block the view, but to instead allow her to be able to hold it lower and thus make it easier to compare the real view with the simulation scenery displayed on the screen. This slight vertical displacement of the correspondence between the two perspectives has proven to function well with users. The horizontal correspondence between the two perspectives, however, remains intact. For the sake of the 
illustrations in this chapter we deactivated tilt offset when taking pictures of a typical user sequence (see Figures 12.4-12.7).

In the following we exemplify a typical user sequence by means of photos taken of the sitsim in use on location and accompanied by explanatory captions and summaries. The new museum and its alabaster-colored hall can be seen to the right. From this view the old building is certainly not dominated by the new building (Figure 12.4). The highest section of the forum artis is clearly subdued by the two towers of the Oslo West Station, despite the fact that the real difference in altitude is more than ten meters. This is dramatically different from the perspective in the public illustrations from the competition and planning process. On moving toward the left, or northern, side of the old museum building, additional details of the proposed structure can be seen (Figure 12.5).

Then, as shown in Figure12.6, the old and new buildings can be seen; the stone wall of the new one directs one's eyes toward the atrium of the old. Next, while still standing on the ground and with the positioning locked, by using the fly-in function one can ascend to the Munch Gallery, and also tilt and pan the phone to look around the simulated or reconstructed room (Figure 12.7).

\section{Trial and evaluation}

As part of a Master's course in Media Innovations aimed at international students at the Department of Media and Communication, University of Oslo, we organized a trial and evaluation of the NasMus-simulation in early March 2012.

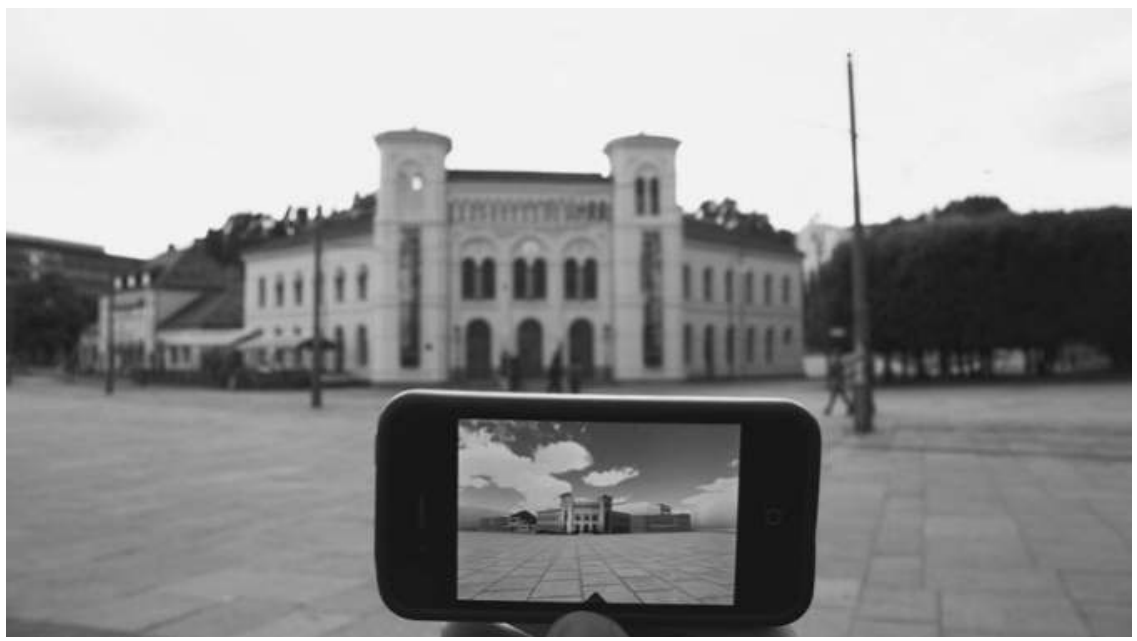

Figure 12.4 The double perspective as viewed from the starting point at a distance of about 75 meters and facing the old building of the museum (@ G. Liestøl). 


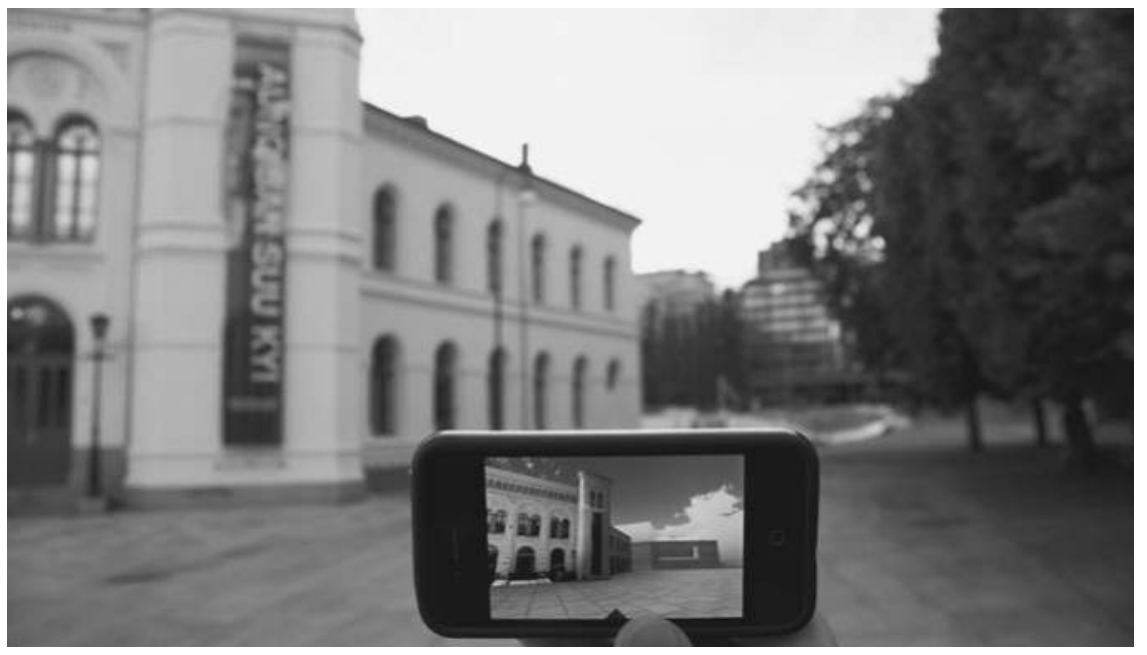

Figure 12.5 Moving toward the left (northern) side of the old building more details of the planned museum appears (@ G. Liestøl).

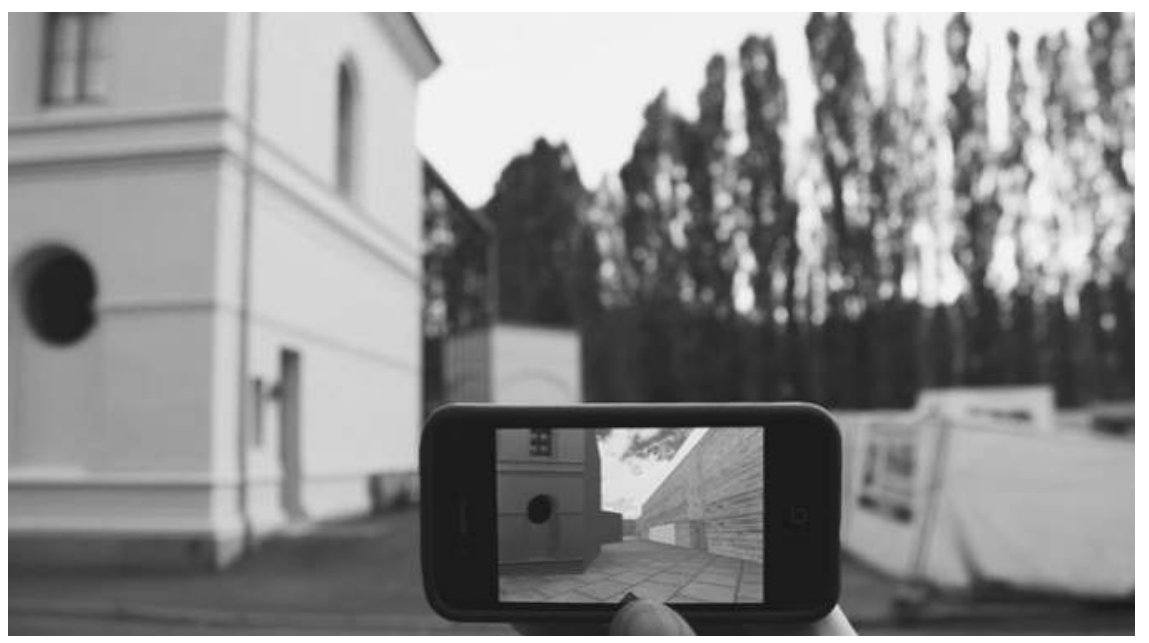

Figure 12.6 Old and new. The stone wall of the new building points in the direction of the main entrance in the atrium behind the old building (C G. Liestøl). 


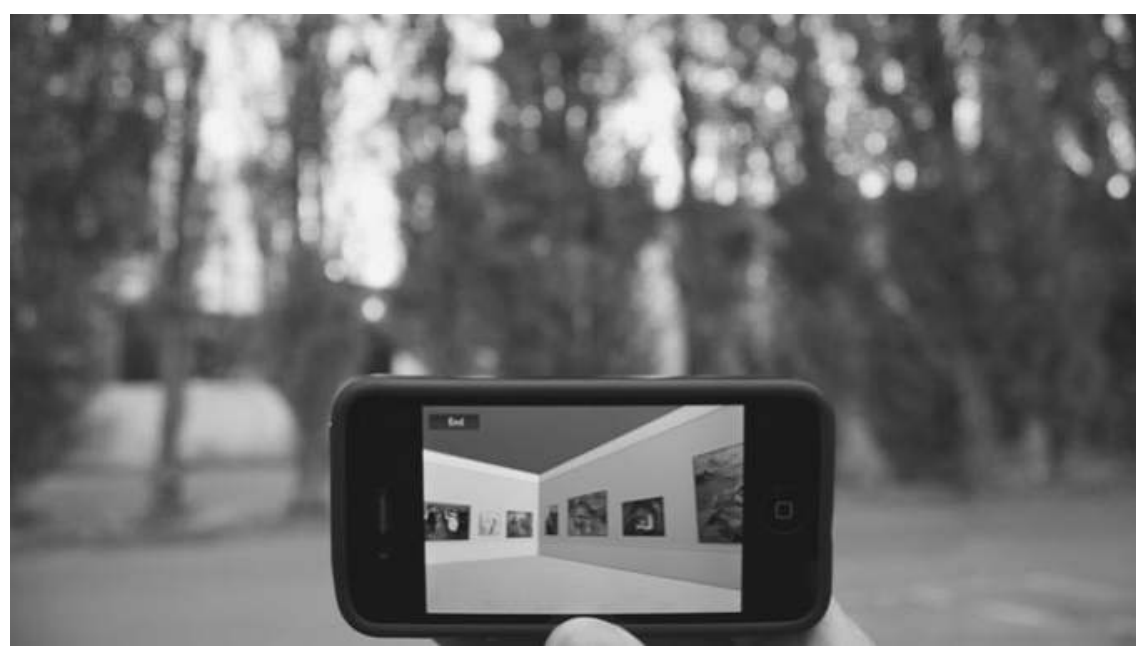

Figure 12.7 Using the fly-in function the user can tilt and pan the device to look around in the simulated Munch room (@ G. Liestøl).

Through a lecture on the university campus the students were first introduced to the general sitsim platform as well as the building project of the new museum. They were shown many examples from the material used to present the forum artis in the competition and for later public dissemination (as mentioned above). They were deliberately not given or shown any information about the NasMussitsim itself before actual testing on location.

On location we selected a starting point at which the students were each given an iPad2. They were encouraged to walk around and view the planned National Museum from different perspectives and positions. They were also informed that there were two links they could activate for access to additional information: the project's website and the Munch Gallery. The students spent about fifteen to twenty minutes each trying out the simulation. When they returned to the meeting point they each answered a written questionnaire with nine questions.

In total ten students (three male and seven female) participated in the trial and handed in the written forms. Their ages ranged from twenty-three to thirty-one. In this group 70 percent had their own touch device, smartphone or tablet. Half of these were iOS devices, the other half were Android. All found the application and its interface fun and easy to use. They liked best the fact that another dimension could be visualized in parallel with the real environment. Many also favorably mentioned the possibility of accessing the Munch Gallery. Two students noted that they felt a bit too much drawn to the screen and thus "forgot" the real environment. One noted the fact that the GPS positioning had problems when you moved too close to the walls of the existing building. Another student wanted a mixed reality solution so that it could be possible to toggle between 
virtual and real perspectives on the screen. Some also wished that a larger part of the surrounding environment could have been included as part of the simulation.

Asked to compare the visual presentations of the Museum project given in the lecture with the in situ use of the sitsim, all favored the use of the on-location simulation. When asked directly if they found the new museum building more or less dominating than expected prior to testing the app onsite, as compared to the information they had after the lecture, all the group agreed that the new building looked less dominating than they had expected.

\section{Conclusion and further research}

We have shown that position and perspective as mediated by use of the sitsim platform - a form of indirect augmented reality - makes a difference in the users' perceptions of the proposed architecture. The fixed camera perspective (still or moving) based in traditional display conventions (Figures 12.2 and 12.3) is in opposition to the free perspective and movement of the virtual camera controlled by the mobile user. This indicates a shift in the visual culture and practice of urban design and planning.

In our work with a variety of sitsims on different topics, information that was included by means of audio and written material, as well as reconstructions of actions and events, have been highly appreciated. There can be no question, though, that the immediate benefit for users with this form of representation and mediation is the active combination of the two perspectives, the real and the computer generated, on location and from the user's subjective point of view. The oscillation between these double descriptions generates new knowledge and added value that cannot be reduced to the two perspectives individually. With past topics this is a question of enriching a historical site with an added dimension that interacts with and improves the aura of the site (MacIntyre et al. 2004). With future topics it is different. This is no longer a question of reconstruction of a return or revisit to something that once existed; rather it is a question of preconstruction, of potential or imminent change and presenting the new. How may this turn be used in urban design? We have seen that the street-level perspective and position of the sitsim user influences the experience of a future construction in different ways than traditional illustrations. How may the subjective perspective play itself out in the hands of the public as part of future planning processes?

"Renderings" such as those we include above in the form of a sitsim applied to the to-be-built environment are already being taken up in architects' offices in parts of Europe, and perhaps further afield. It is likely that augmentation such as we present above will be included in the array of tools and mediational means architects employ in competitions, potentially tools in the hands of planners, students of design, and the marketing of real estate more broadly. Cultural competitions may soon be infused with sitsim-like contributions that will add an additional rhetorical and persuasive layer of semiosis. They too will need to be 
more closely studied in terms of their perceived and actual use over time in relation to positioning and location-centric views (cf. Bates-Brklajac 2009).

With the rapid spread of location-based functionalities, locative apps on smartphones and tablets have already been widely taken up in highly developed metropolitan areas. They are increasingly connected to our own situated uses and locative mappings as urban inhabitants. Sitsim-like renderings may well become a more significant feature of the wider communicative and persuasive inscription of locative media within architectural discourses. For the time being, it may well be that this is most apparent in mediations of future structures and their presentation earlier on in processes of urban change, regeneration, and gentrification.

The context and orientations of sitsims may also open out spaces for wider public discourses and locative engagement in projections of the future urban landscape, the types of buildings and their purposes and uses (Al-Kodmany 1999). As a locative, mediational, and multidimensional genre prototype, the sitsim offers us a potentially navigable platform but also a site for the performative annotation of our perceptions and interpretations. In the development of our sitsims we have taken this from historical Viking burial sites where cultural artifacts are not visible in the Norwegian countryside to the physical remains of the Forum in the middle of today's Rome. In the case we report on here, we have moved this platform into the current urban fabric of processes of inviting, selecting, and projecting a significant new national museum in a capital city. In so doing, this sitsim nevertheless both incorporates and is dependent on contextualization in the physical world of the here-and-now. Yet, simultaneously, reference is also made to digital spatialization that can be annotated. This functionality gives future potential for the sitsim to also be actively taken up as part of the wider communicative and mediated negotiation of power, place, and participation in the city. We may see this as part of what Thrift (2004: 187) views as "new conditionings of position and juxtaposition." This is increasingly what we see as a networked city (Martinussen 2013) where layers of tools and representations may be linked in our contexts of activity and thereby span communication types, data visualizations, social media, and augmented locative designs and technologies. For Thrift (2004: 188), however,

modern complex systems are so overdetermined that in their interleavings all kinds of gaps are likely to be found in which new kinds of "excursions" can be coaxed into existence. If things are showing up differently, we can do different things too, energetically opening up the new order of being. As the direction of attention changes, so perhaps, we make a change in the direction of our attention, sensing possible emergences and new embodiments.

However, these emergences, embodiments, and mediations need to be revised in terms of what they selectively represent, how they convey the spatial, visual, temporal, and sensorial, and what aspects they accentuate, augment, and filter via such tools and translations. 
What we have shown is how we might approach the locative, mobile, and culturally contextual through hands-on design-centered innovation lodged in cocreative development that involves computational, cultural, and communicative knowledge that also needs to be critically examined further to escape the potential for locative functionalism or the mere generation of a form of mobile facades.

Design and development on the National Museum sitsim continues and in the late fall of 2012 a new more extensive version was published for free download on Apple App Store and Google Play. In this version users on location can give feedback directly via the sitsim from specific positions and thus compare their own experience of the new structure with other users, and the judgment of the jury from the last competition. This feedback will be analyzed and used for further designs and discussions of how employments of place, position, and perspective may be facilitated in continued development of sitsims and other mobile augmented reality solutions in urban planning.

\section{Acknowledgements}

Thanks to collegues in the SITSIM research and development process and the Department of Media and Communication, University of Oslo. Special thanks to Tomas Stenarson at CodeGrind for programming and collaboration on the design and to Šarūnas Ledas at Tag of Joy for 3D-modelling. Thanks also to Arvid Esperø and Diderik Haug at Statsbygg, and to Klaus Schuwerk at Kleihues + Schuwerk.

\section{Notes}

1 Currently, the most updated version of the 'Roman Forum' sitsim is available as a free download on Apple's App Store and Google Play.

2 See: www.statsbygg.no/Utviklingsprosjekter/Nasjonalmuseet/Nasjonalmuseet-paVestbanen/.

\section{References}

Al-Kodmany, K. (1999) Using visualization techniques for enhancing public participation in planning and design: Process, implementation, and evaluation, Landscape and Urban Planning, 45(1): 37-45.

Azuma, R. (1997) A survey of augmented reality, Presence: Teleoperators and Virtual Environments, 6(4): 355-385.

Azuma, R., Y. Baillot, R. Behringer, S. Feiner, S. Julier, and B. MacIntryre (2001) Recent advances in augmented reality, IEEE Computer Graphics and Applications, 21(6): 34-47.

Bates-Brklajac, N. (2009) Assessing perceived credibility of traditional and computer generated architectural representations, Design Studies, 30(4): 415-437.

Bratton, B. (2009) iPhone city, Architectural Design, 79(4): 90-97.

Büscher, M., J. Urry, and K. Witchger (2011) Mobile Methods, Abingdon: Routledge. 
Chupin, J.-P. (2011) Judgement by design: Towards a model for studying and improving the competition process in architecture and urban design, Scandinavian Journal of Management, 27(1): 173-184.

Donald, J. (1999) Imagining the Modern City, London: The Athlone Press.

Dunn, N. (2010) Architectural Modelmaking, London: Lawrence King.

Farman, J. (2012) Mobile Interface Theory: Embodied Space and Locative Media, Abingdon: Routledge.

Felix, N., J. Torpus, and M. Wiedmer (2008) Negotiating reality, n Proceedings of Undisciplined! Design Research Society Conference, Sheffield Hallam University, Sheffield, UK, July 16-19, available at: http://shura.shu.ac.uk/521/.

Gordon, E. and A. de Souza e Silva (2011) Net Locality: Why Location Matters in a Networked World, Chichester: Wiley.

Hah, E., P. Schmutz, A. N. Tuch, D. Agotai, M. Wiedmer, and K. Opwis (2008) Cinematographic techniques in architectural animations and their effects on viewers judgment, International Journal of Design, 2(3), available at: www.ijdesign.org/ojs/index.php/ IJDesign/article/view/479.

Hjorth, L., J. Burgess, and I. Richardson (2012) Studying Mobile Media: Cultural Technologies, Mobile Communication, and the iPhone, Abingdon: Routledge.

Klett, M. with M. Lundgren (2006) After the Ruins. 1906 and 2006. Rephotographing the San Francisco Earthquake and Fire, Berkeley, CA: University of California Press.

Kreiner. K., P. Jacobsen, and T. Jensen (2011) Dialogues and the problems of knowing: Reinventing the architectural competition, Scandinavian Journal of Management, 27(1): 160-166.

Liestøl, G. (2011) Situated simulations between virtual reality and mobile augmented reality: Designing a narrative space, in B. Furht (ed.), Handbook of Augmented Reality, New York, NY: Springer, pp. 309-319.

Liestøl, G. and A. Morrison (2013) Views, alignment and incongruity in indirect augmented reality, in Proceedings of ISMAR 2013, IEEE International Symposium on Mixed and Augmented Reality-Arts, Media and Humanities. October 1-4, pp. 23-28, available at: http://ieeexplore.ieee.org/xpl/ articleDetails.jsp?tp=\&arnumber=6671263 \&queryText $=$ Liest $\% \mathrm{C} 3 \% \mathrm{~B} 81$.

Liestøl, G., A. Doksrød, S. Ledas, and T. Rasmussen (2012) Sensory media: Multidisciplinary approaches in designing a situated and mobile learning environment for past topics, International Journal of Interactive Mobile Technologies, 6: 18-24 (open access), available at: http://online-journals.org/i-jim/article/view/2097.

Liestøl, G., A. Morrison, and T. Rasmussen (eds.) (2003) Digital Media Revisited, Cambridge, MA: MIT Press.

Liestøl, G., T. Rasmussen, and T. Stenarson (2011) Mobile innovation: Designing and evaluating situated simulations, Digital Creativity, 22(3): 172-184.

Lynch, K. (1960) The Image of the City, Cambridge, MA: MIT Press.

MacIntyre, B., J. Bolter, and M. Gandy (2004) Presence and the aura of meaningful places, in Proceedings of Presence 2004, Valencia, Spain, October 13-15, pp. 36-43, available at: http://ispr.info/presence-conferences/previous-conferences/ presence2004/.

Martinussen, E. (2013) Pockets and cities: Design and popular imagination in the networked city, Design and Culture, 5(3): 289-312.

McCullough, M. (1998) Abstracting Craft: The Practiced Digital Hand, Cambridge, MA: MIT Press. 
McCullough, M. (2004) Digital Ground, Cambridge, MA: MIT Press.

McLuhan, M. (1964) Understanding Media: The Extensions of Man, New York, NY: McGraw Hill.

Meredith, M., Aranda/Larsch and M. Sasaki (eds.) (2008) From Control to Design: Parametric/Algorithmic Architecture, Barcelona: Actar.

Milgram, P. and F. Kishino (1994) A taxonomy of mixed reality visual displays, IEICE Transactions on Information Systems, E77-D(12): 1321-1329.

Morrison. A. and H. Mainsah (2012) Building communication by design: Mobile fiction and the city, in M. Hensel (ed.), Design Innovation for the Built Environment: Research by Design and the Renovation of Place, Abingdon: Routledge, pp. 221-234.

Morrison, A. and S. Skjulstad (2010) Unreal estate: Digital design and mediation in marketing urban residency, in I. Wagner, T. Bratteteig, and D. Stuedahl (eds.), Exploring Digital Design, Vienna: Springer, pp. 189-219.

Pierroux, P. and S. Skjulstad (2011) Composing a public image online: Art museums and narratives of architecture in web mediation, Computers and Composition, 28(3): 205-214.

Robbins, E. (1994) Why Architects Draw, Cambridge, MA: MIT Press.

Rönn, M. (2011) Architectural quality in competitions. A dialogue based assessment of design proposals, FORMakademisk, 4(1): 100-115, available at: http://journals.hioa. no/index.php/formakademisk/article/view/130.

Schnädelbach, H. (2009) Visibility in architecture extended through audiovisual communication technologies, in D. Koch, L. Marcus, and J. Steen (eds.), Proceedings of the 7th International Space Syntax Symposium, Stockholm: KTK, pp. 1-13.

Thrift, N. (2004) Remembering the technological unconscious by foregrounding knowledges of position, Environment \& Planning D: Society and Space, 22(1): 175-190.

Tostrup, E. (2010) Promoting the best: On competition rhetoric, in M. Rönn, R. Kazemian, and J. Andersson (eds.), The Architectural Competition, Stockholm: Axel Books, pp. 77-96.

Verhoeff, N. (2012) A logic of layers: Indexicality of iPhone navigation in augmented reality, in L. Hjorth, J. Burgess, and I. Richardson (eds.), Studying Mobile Media: Cultural Technologies, Mobile Communication, and the iPhone, Abingdon: Routledge, pp. 118-132.

Williams, A. and P. Dourish (2006) Imagining the city: The cultural dimensions of urban computing, Computer, 39(9): 38-43.

Wither, J., J.-T. Tsai, and R. Azuma (2011) Mobile augmented reality: Indirect augmented reality, Computer and Graphics, 35(4): 810-822. 


\title{
The will to connection
}

\author{
A research agenda for the \\ "programmable city" and an ICT \\ "toolbox" for urban planning
}

\author{
Ole B. Jensen
}

\begin{abstract}
The people who first built a path between two places performed one of the greatest human achievements. No matter how often they might have gone back and forth between the two and thus connected them subjectively, so to speak, it was only in visibly impressing the path into the surface of the earth that the places were objectively connected. The will to connection had become a shaping of things, a shaping that was available to the will at every repetition, without still being dependent on its frequency or rarity. Path-building one could say, is a specifically human achievement; the animal too continuously overcomes a separation and often in the cleverest and most ingenious ways, but its beginning and end remain unconnected, it does not accomplish the miracle of the road: Freezing movement into a solid structure that commences from it and in which it terminates.
\end{abstract}

(Simmel 1909: 66)

Contemporary cities must be understood as complex assemblages of sociotechnical networks, built environments, and human subjects. With the advent of networked technologies and increasing physical and digital mobility new perspectives and theories are in demand (Brynskov et al. 2012; Castells 2005; Farman 2012; Gordon and de Souza e Silva 2011; Elliott and Urry 2010; Farias and Bender 2010; Jensen 2013; Jensen and Thomsen 2008; Sheller and Urry 2006; Shepard 2011; Vannini et al. 2012; Wilken and Goggin 2012). Kitchin identifies the "programmable city" as a research agenda engaging with this new complex relationship between technologies, software and the city (Kitchin 2011). In this chapter I address the fields of urban planning and design and their needs for mastering networked technologies. This is done by discussing what an "Information and Communication Technology (ICT) Toolbox" for urban planners and designers might mean. A planners' and designers' "toolbox" should contain three dimensions: hardware (technological devices), software (operative code), and a manual (here understood as a theoretically informed conceptualization of the socio-cultural embedding of these networked technologies). So the argument put forward in this chapter is that there is a need for creating operational tools and understandings (or what I term manuals) that urban planners and 
designers may utilize next to the technologies and software. The theoretical background for this is derived from the "mobilities turn" (e.g., Cresswell 2006; Urry 2007) as well as theories exploring network technologies and new media (e.g., Farman 2012; Gordon and de Souza e Silva 2011). To frame this agenda I refer to research undertaken at the cross-disciplinary Centre for Mobilities and Urban Studies (C-MUS) as well as in the Research Cluster for Mobility and Tracking Technology (MoTT) both located at Aalborg University, Denmark.

The chapter is structured in six sections. After the introduction, I discuss networked technologies as part of a research agenda for the "programmable city." The following section introduces the two institutional settings for mobilities research with a reference to the programmable city research agenda. In section four, the chapter addresses implications for practice. In this case the discussion is about the need for urban planners and designers to relate to the new situation of networked technologies, and the chapter argues for the need for an ICT toolbox dealing with these issues. Next, I sum up the contributions to the research field. The final section contains concluding remarks and perspectives for future research.

\section{Networked technologies and the "programmable city"}

The manifold ways that new networked technologies influence contemporary urban life lie beyond the scope of this chapter (for some of this story see: Crang and Graham 2007; Farman 2012; Gordon and de Souza e Silva 2011; Graham 2010b; Jensen 2013; McCullough 2004; Wilken and Goggin 2012). Rather I want to approach this by way of discussing a research agenda that targets these new technologies very directly. I am thinking of Kitchin's notion of the programmable city and how that research agenda may fit the aim of this chapter. Kitchin argues that when analyzing the relationship between software and the city, there are two important distinctions to be made (Kitchin 2011: 946). One is termed "translation" and has the focus of how cities are translated into code. The other is termed "transduction" and raises the question of how code reshapes city life. Within each of these dimensions a subset of questions arises. In the dimension of translation we may ask how to understand the city as digital data are generated and processed. We may inquire about managing the city in the sense of looking at how city government discourses and practices are translated into code. We may explore how the geography and political economy of software production shape various coding practices. Finally, the theme of "translation" points at the issue of how software becomes legitimated and discursively produced by power and vested interests. The second dimension termed "transduction" equally gives rise to a set of questions. How does software drive public policy in both its implementation and development? In managerial terms, how is software regulating and governing urban life? In terms of work, how does software alter the nature of work and its urban expression? And finally how does software work to transform the material and spatial dimensions of cities? 
The complex issues of the research agenda for the programmable city cannot be addressed in this chapter. But I find them illustrative of some of the challenges that cross-disciplinary research environments such as C-MUS and MoTT need to engage with in the very near future. Furthermore, some of them are directly relevant to the toolbox discussion. The toolbox should contain hands-on technologies and hardware (e.g., cameras, GPS transmitters, RFID tags, etc.), software and code to operate these (e.g., custom-made apps for smartphone tracking), and finally what I would term manuals as shorthand for the theoretically informed knowledge about the social and cultural embedding of these technologies. Earlier on Kitchin and Dodge pointed at the fact that code, software, and the various hidden operative systems of networked technologies are neither neutral nor easy to escape. They coined the notion of "code/space" precisely to point out the interrelatedness and complex hybridization between code and space, the material and the virtual, the physical and the digital (Kitchin and Dodge 2011). From this rather general discussion of the programmable city I want to turn toward two specific examples of research within the realm of such thinking.

\section{C-MUS and MoTT: exploring the programmable city agenda}

Even though Kitchin's research agenda for the programmable city has been articulated in a different context, I would argue that it actually summarizes parts of the mobilities research at Aalborg University quite well. So in this section I briefly describe the Centre for Mobilities and Urban Studies (C-MUS) and the research cluster for Mobility and Tracking Technologies (MoTT) as institutional arenas where many of the research questions raised by Kitchin are actually engaging various researchers from across many different disciplines. I am confident in using these two cases for this discussion since I am a co-founder and board/task force member in both of these institutions. Kitchin's questions, moreover, are very precise in pointing at what I believe will be the urban planner's and designer's need for knowledge in relation to ICT.

\section{The Centre for Mobilities and Urban Studies (C-MUS)}

C-MUS was set up in 2008 and works across the Faculties of Social, Human, and Technical Sciences. It has taken on the "mobilities turn" as a framing perspective in a pragmatic and explorative sense, and has over the years produced results within fields as diverse as communication and culture, urban planning, traffic engineering, urban sociology, and geography to mention but a few.

In general terms the research within C-MUS mainly aims at the transductive dimension of Kitchin's research agenda (even though there are projects falling within the translation dimension as well). But in relation to Kitchin's research agenda for the programmable city, I would indicate three research dimensions of 
particular relevance. One dimension is related to planning, policy making, governing, and orchestrating mobilities. Another relates to mundane everyday practices of mobilities and their socio-cultural embedding. The third area turns toward communication technologies and their facilitation and affordance of various forms of mobilities (physical, as well as virtual). Most importantly for the discussion of the programmable city and a planners' and designers' toolbox is, however, the fact that all this research is carried out in cross-disciplinary settings by researchers with such diverse backgrounds as engineering, sociology, planning, culture and communication studies, and urban design.

\section{The research cluster for Mobility and Tracking Technologies (MoTT)}

I shall move on to a smaller group established in 2011 under the Department of Architecture, Design, and Media Technology named Mobility and Tracking Technology (MoTT). MoTT is more dedicated and focused to the research agenda of the programmable city and one might in fact speculate whether we might have used Kitchin's ideas more directly had we known these prior to forming the research cluster. In MoTT there are media technology researchers with backgrounds in engineering, software development, computer vision, etc. mixed with researchers from urban design and architecture. Accordingly MoTT focuses on five key challenges (see web link at the end of the reference list for this chapter):

1 Exploring situational human-technology interaction concerning multiple human sense modalities. For example, within software for facial recognition used in surveillance systems.

2 Developing and testing monitoring and tracking technologies within a wide range of approaches (GPS, RFID, Bluetooth, Infrared scanning, etc.). For example, tested in urban design and mobility planning contexts.

3 Contributing to theoretical explanation models and concepts capturing the societal and technological importance of monitoring and tracking technologies. For example, cross-disciplinary theory building of mobility, monitoring, and tracking technologies.

4 Exploration of new visualization techniques and tools in order to capture mobilities within monitoring and tracking technologies. For example, by making new models of mapping and visualization techniques.

5 Exploration of the normative, cultural, and ethical repercussions for a society increasingly embedded with mobility monitoring and tracking technologies.

With this institutional framing we move closer to the translation dimension of Kitchin's agenda. Again this is in general terms since there are issues such as items 4 and 5 from the above list that are more related to the transductive dimension. My 
main point here is, however, that C-MUS and MoTT foreground the two dimensions of the programmable city with different emphasis. Another key difference that might be said to exist is that the research efforts undertaken by MoTT are more technologically driven and thus more focused on the translation dimension with its awareness of the way networked technologies are shaping and affording mobilities, looking both "inward and outward" so to speak. In other words, the MoTT research perspective takes its point of departure in the hardware and software that is currently "out there" and explores technologically how these may be altered, improved, or customized. The issues of the societal embedding and the ethical discussions for instance are, however, important. The MoTT research agenda is of relevance to all three toolbox dimensions as it engages with the hardware and software in a very hands-on fashion, as well as being occupied with exploring what might be the adequate theoretical underpinning; and thus is concerned with how a theoretically informed manual exploring both the social and ethical implications of a given networked technology might take shape.

From briefly illustrating how the programmable city research agenda might become institutionalized I want to move toward the more concrete discussion of the relationship between the need for tools and the actual research across these institutional settings.

\section{ICT toolbox for urban planners and designers}

The need for an ICT toolbox reaches across all disciplines engaged in the complex endeavor of planning and designing cities. City planners, architects, urban designers, and various engineering fields thus need to factor in the meaning of networked technologies and their repercussions for contemporary urban life (see Huang 2012 for an account of an ICT urban planning strategy in Taipei). In Denmark there is a beginning multidisciplinary and cross-professional practice related to urban design and planning. This is only in its infancy and many municipal planners and urban designers working out of architectural companies still cling to their professional identities as relatively isolated and at times even insulated professions. Municipal organizations often contribute to such insular perspectives by organizing e.g., "traffic" within a dedicated Traffic or Technical Department and e.g., "urban space design" within a Planning Department. Obviously these are intimately connected and thus should be thought of across organizational and professional lines of division. This is not a new situation, but with the advent of location-based technologies, mobile communication devices and networked technologies, the blurring of physical/digital, local/ global, and visible/invisible makes the upholding of strict lines of demarcation even more problematic. If we add to this a situation in which a fairly large proportion of the planners, designers and architects are reaching the age of retirement, there is a technological "generation gap" as well. Surely some professionals dealing with urban planning and design realize that new networked technologies are part of the city as well as being potential new tools for 
"reading" the city. But there is still a climate of suspicion and even moral fear related to these technologies that very often are associated with surveillance and "Big Brother" dictatorship.

At a recent meeting with municipal planners in a Danish city I experienced this rather directly. After the meeting a group of us were sitting in the Technical Department's lunch canteen. I was seated next to an architect urban planner, who spontaneously reacted to my telling her about our research into GPS tracking by saying: "yes, these are scary." Obviously we may be scared by new technologies and there are plenty of gloomy scenarios (see e.g., Graham 2010a), but it struck me as something of a problem if planners and urban designers only tap into the "dark side" (Jensen 2013) understanding of these technologies. This was just one incident and thus not enough to describe a full field of practice. However, in a recently published article targeting urban planners in Denmark, Jørgensen points to the same problem as something of a much more general nature:

It is widely believed that personal media technologies remove us from what is important. This idea is based on the assumption that meaningful relationships can only occur in face-to-face meetings. Such an approach leads to urban planning strategies that focused solely on physical space, and neglecting the virtual. I do not agree with that assumption. In modern society personal media such as smartphones are the interface between our social networks and the physical world. When this interface is mobile and contextsensitive, we can weave together these various spaces in new ways. It adds another dimension when the city begins to "respond" to its citizens, through more or less visible technologies embedded in urban spaces. Examples of this can be when traffic lights are optimized for traffic surveillance, cameras track criminals, and pollution sensors and dynamic signage allows you to divert heavy traffic. You could say that the city itself to a far greater degree than previously is to be understood as a living, sentient entity.

(Jørgensen 2011: 19, my translation)

So to return to the introductory quote from Georg Simmel, we might want to enquire how the "will to connection" is played out in cities among networked technologies and city dwellers. But we may focus this question and narrow it even further to start thinking about the urban planners and designers and their "will to connection." How do urban planners and designers embrace, engage with, or resist networked technologies? This is an issue worthy of its own fullfledged research agenda. Here I confine myself to stipulating how research such as the work done in C-MUS and MoTT engages with facilitating and disseminating knowledge related to the technologies in the toolbox. I think it is safe to say that the networked technologies ought to interest all planners and urban designers for at least four reasons. First of all because they are simply "out there working" as we speak. This means, like it or not, that networked technologies are as much a part of the city as roads, pavements, city plazas, and buildings. 
Any designer or planner concerned with the city should therefore mobilize an interest in these technologies if they claim to know the city. Second, the technologies at work are producing data about the city that urban planners and designers may as well make use of in their attempts to monitor and track the city and its dynamics (setting aside the at times rather subtle legal issues related hereto). Third, and this may appeal to many process-oriented planners, these technologies offer new opportunities for engaging with the public, to facilitate new public participation processes and involve segments of the city's population that are not normally very vocal in urban planning processes. There is a wider discussion about digital divides and age groups to be taken into account here. Some seem to think that applying new networked technologies in public participation processes excludes the "digitally illiterate" and the senior segments of the population. However, if these technologies are used in supplement to existing and wellproven methods of citizen involvements like planning charrettes and public hearings, I would rather claim that the toolbox has been supplemented with new tools adding to the competencies rather that substituting one exclusion with another (the "old" participation tools may be said to exclude the younger segments of the population as well). This debate should call for more investigation and research really to be substantiated.

The fourth and final reason why this is important to urban planners and designers is that the new networked technologies increasingly are being explored by artists and creative designers testing out how technologies may afford new aesthetic sensations, cultural interchanges, social and playful experiences. I want to term this field of technology application within urban planning and design "performative urban spaces." This refers to the way networked technologies in material spaces may start "working" and perform as a consequence of people's engagements. This fourth level might be the most complex level of the toolbox, but nevertheless a field where the public is already experimenting with various playful projects and technologies (Gordon and de Souza e Silva 2011; Jensen and Thomsen 2008).

The research undertaken in C-MUS and MoTT targets the translation and the transduction dimensions, as well as engaging with all four reasons for paying attention to the development of networked technologies. From here I present a short overview of C-MUS and MoTT research.

\section{GPS, GIS, SMS, and RFID}

Researchers have undertaken a large number of projects using GPS technologies to map and track users of anything from inner city spaces, to urban parks and zoological gardens. The research has worked on bridging the use of dedicated GPS devices (prior to the advent of smartphones), GIS, and various interview and survey techniques. However, lately the development of apps for smartphones has entered the field of research, as well as a new approach linking GPS data to SMS messages. This latter dimension has been an attempt to capture people's 
immediate sensations and impressions as they move in the city (Reinau et al. 2012). The research furthermore has been visualized in various forms of GIS maps and other digital representation tools. Some of the research projects have been focusing on the way GPS tracking may be used by neighborhood residents to "draw their city with their feet" and thus open up to new methods of public participation (Harder et al. 2012; Knudsen et al. 2011; Nold et al. 2008). The toolbox technologies are here very much about "capturing mobilities" and mapping the activities in the city. Moreover, some of them lend themselves to particular framings and ways of re-presenting flows like GIS maps as "heat maps" showing various forms of mobile geolocated intensities. The SMS research together with the participatory explorations of apps in community mapping projects are illustrative of the dimension of the toolbox where the technologies are not only mapping "what is there" but may be proactively used in citizen participation processes, e.g., in identifying sites or routes of particular interest and quality (or the opposite). Recently a project utilizing radio frequency identification (RFID) has been applied to indoor locations. Through the use of RFID research has tracked user occupancy in public buildings and cultural houses (Suenson 2012; Suenson and Harder 2011). This research might move toward open spaces and city spaces as a next phase. This is research in its very early phases, and the work undertaken in these projects has been done indoors due to various constraints to the technology. However, larger urban zones may be equipped with RFID transmitters and receivers, such as parking payment zones.

Geolocation tracking and real-time data collection are crucial as new tools for urban planners and designers exploring the whereabouts of the citizens, the usage of urban spaces, as well as the new potentials of areas and sites in the city.

\section{Camera tracking}

Much of the literature on uses of cameras in urban and public spaces is about the ethics of surveillance and how the various closed circuit television (CCTV) systems are often utilized in relation to crime prevention schemes. In the examples I mention here the types of cameras are, however, of another type. Utilizing heat-sensitive (thermo) cameras provides an advantage in a Danish context where the regulations for surveillance of public space are fairly strict. This means that getting permission to utilize cameras in public spaces and various measures to secure privacy for people being filmed can be both time and resource consuming. In cases where the research question does not require individual identification, the use of heat-sensitive cameras solves the problem since personal identity is safeguarded as one cannot see faces or other identifying features on these representations. I want to mention a project utilizing thermo cameras but also point to research done with much more sophisticated technologies for facial recognition and social detection (e.g., Social Signal Processing or "SSP," see Moeslund 2012). But these technologies are yet in their infancy and 
will not reach urban planners' and designers' toolboxes for a considerable time to come.

The so-called Kennedy Project (named after the plaza where it took place) was a project detecting people's movement across a transit space in front of the rail station in Aalborg, Denmark. There was a complicated relationship to the lighting at the square that I will elaborate on below, but in terms of camera tracking the important thing to notice was that by utilizing heat-sensitive cameras we obtained data on the movement patterns and flow rhythms without jeopardizing people's right to privacy. The camera was fixed to a building wall many meters above the square and produced fairly reliable data (Poulsen et al. 2012a). For now the use of ordinary CCTV type of camera as well as heat-sensitive cameras are very relevant tools for urban planners and designers, and these technologies potentially provide important information about real-time usage of streets, squares, and public spaces in the city. In a longer term perspective the technologies of "Social Signal Processing" (SSP) where people may be identified by facial expressions or bodily gestures will become more prevalent and of interest to systems of security and police monitoring.

\section{Performative urban spaces}

I want to end this very brief list of examples by pointing out how the "ordinary" tracking technologies may in fact be inserted into projects wherein there are even artistic ambitions. These are examples that reach beyond ordinary ICT uses and point toward experiential city design (Marling et al. 2009). Many of the described technologies are applicable to these types of more artistic projects where the focus is on enhancing the experience of new types of public spaces and playful interaction with networked technologies. These projects are often more complex than many of the abovementioned explorations of technologies, but may be understood as an important level of planner and designer ICT toolbox application. So in addition to the three first reasons why urban planners and designers should pay attention to the new networked technologies, there is the goal of rearticulating the meaning of public spaces and the creation of new playful installations in the city. These are part of the global trend toward rethinking inner city public spaces as "scenes" for social and cultural interaction and public domain (Hajer and Reijndorp 2001; Gordon and de Souza e Silva 2011; Jensen and Thomsen 2008; Marling et al. 2009).

I want to return to the Kennedy Project since besides using heat-sensitive cameras as described above this project also went into interaction with people crossing the public square by turning up and down the lights as well as shifting colors depending on the number of people and their routes crossing the square (Poulsen et al. 2012a). The lesson of interest to this chapter is that the KennedyProject showed how important not only technical skills are (calibrating cameras, software, lamps, etc.) but also how to reflect about the movement patterns and people's understanding of an urban transit space. In other words, next to the 
hardware and code that were absolutely necessary, this project also illustrates that social knowledge about people's movement patterns as well as their preconceptions of urban transit spaces are important features of a toolbox (the dimension I term manuals). During the last couple of years other similar projects have been made in Aalborg. For example, the Red Pavilion was an installation screen at the harbor front, changing lights in accordance with music played and people interacting (Poulsen et al. 2012b). Also the Nora Project was aimed at setting up a feedback loop between people's presences and movements through a pavilion where both sound and color were reflecting the patterns of human movement (Jensen and Thomsen 2008). At the more exotic end, I want to end this listing with a project in which a robot dressed as Santa Claus moved about inside the transit node of the Kennedy Arcade (Tranberg et al. 2009). The robot identified, tracked, and followed people, while a set of research assistants observed and interviewed people about the experience. The project gave insights into the very technical discussion about robot calibration and laser range finder sensitivity, as well as issues of what a robot in public spaces might mean to people, how they perceive transit spaces, and how these may be challenged as instrumental transport spaces only.

The list could be extended significantly as well as accompanied by much more thorough explanations to do justice to the research projects. Here I merely have intended to illustrate that within C-MUS and MoTT there are projects of relevance to the ICT toolbox of urban planners and designers. What would be interesting to investigate in more depth in technological terms are $\mathrm{WiFi}$ and Bluetooth technologies as well as more dynamic visualization techniques.

\section{So what's in the toolbox? Contribution to an emerging research field}

I like to think of these networked technologies as both related to hardware, software, and manuals. In other words the toolbox needs to contain hardware technologies such as GPS trackers, CCTV, heat-sensitive cameras, RFID tags, GIS maps, 3D dynamic visualizations and animations, and many other forms of code, software, and hardware. However, this is not enough to comprise a reasonable and operational toolbox. The manual or theoretically informed knowledge about the social and cultural embedding of these technologies is just as important. In other words, next to the many existing software and hardware "out there," urban planners and designers also need to harvest the key insights from the part of the "mobilities turn" literature that concerns itself with the meaning of networked technologies, how they afford or prevent social and cultural practices, and ultimately how the city becomes an assemblage of socio-technical systems, artifacts, flows of people, goods, vehicles, and information in a complex geography of connectivity. The toolbox needs a manual in the sense of a well-informed and theoretically underpinned set of statements and analytical positions on the technologies (which also should contain the ethical issues related to these new 
technologies). The toolbox manual should contain theories and concepts for understanding how these technologies both are present in the city as data producers to be tapped into, as well as they may afford new types of public participation. In this chapter I have used the research within C-MUS and MoTT as examples even though there obviously are many other institutions and areas bringing about knowledge of networked technologies.

The theoretical input for the manual may generally be derived from the "mobilities turn" literature I have listed earlier in this chapter. But to become more specific I would point at the situational and everyday life perspective as a fruitful underpinning of the toolbox manual (see Jensen 2006, 2007, 2009, 2013). Spanning both the translation and the transduction dimensions I believe the manual can put focus on how mobile subjects are being empowered by the new technologies even as these are inscribing new power structures in the everyday life. Moreover, the programmable city research agenda points to the ICT toolbox as being both a rational and instrumental way of providing data about the city, as well as it becomes a new participatory tool and also a new interface between mobile urbanites in their daily practices. Thus the networked technologies reconfigure people's relationships to other people and places, and the way they think of self, other, and the built environment. This huge task of bringing in new technologies in urban planners' and designers' professional practice can of course neither be accomplished by one or two research environments nor can it be dealt with from the point of view of one academic discipline. In this chapter I have, however, wanted to point at ongoing research efforts of relevance to dealing with this task and to point toward the "mobilities turn" as a cross-disciplinary platform from which to launch some of the future activities that may afford the creation of a constructive and professional engagement with networked and mobile technologies in the city. In Kitchin's words:

I have made the case that we are entering a period of programmable urban$i s m$, and that to understand this new form of urbanism we need to examine the various components of how the city is translated into code and how the resulting software is reshaping city life - that we need to understand the internal workings of the black box in order to better understand its external work. In so doing we can start to address a series of important lacunae in understanding and theorizing contemporary urbanism, opening up new comprehensions of the city at a time when urban life is going through profound changes with respect to its organization, scaling, and management

(Kitchin 2011: 950, my emphasis)

\section{Concluding reflections}

In the period of "programmable urbanism" new challenges await all sorts of professions. In this chapter I have wanted to point toward urban planners and designers as a particular group in need of engaging with these technologies. I 
indicated four key reasons why I find this of high importance. First because the technologies are already "out there," urban planners and designers must know about these since they are as important a part of the contemporary city as asphalt, sewers, houses, and public plazas. Second, the networked technologies are producing data about the city that planners and designers might as well make use of to create more accurate and sophisticated decision-making frameworks. Third, the networked technologies (despite all the "digital divide" discussion) offer a potential for new types of public participation processes and civic engagement in urban planning and design. Fourth, the new technologies can do more than map and report the activities in the city. They offer themselves to become active and dynamic layers of new experiences and creative practices of the city. The "performative" dimensions of networked technologies should become tools in the box that also open up new technology-driven discussions about cross-departmental collaboration as well as inclusion of artists and creative agents. Thereby, if it plays out well, there is a potential for using these technologies to open up the fields of urban planning and design toward more creative and artistic dimensions (something I believe these fields would benefit from indeed). The manual helping urban planners and designers to fully grasp the social and cultural implications of the networked technologies should be anchored in the "mobilities turn" literature in general, with a specific focus on the mundane everyday life and its reconfiguration as an effect of these technologies.

The "will to connection" is as old as humans' movements across the surface of the Earth. However, we need to understand that the traces are no longer only visible. Networked technologies are creating new challenges, and urban planners and designers might benefit from expanding their toolbox with important ICT technologies. As mentioned, people are already engaging with these technologies and their mobility affordances regardless of what planners and designers might think thereof. The time has come for urban planners and designers to mobilize their "will to connection."

\section{References}

Brynskov, K., K. Halskov, and L. Kabel (2012) Byens digitale liv/Digital Urban Living, Aarhus: Forlaget Ajour.

Castells, M. (2005) Space of flows, space of places: Materials for a theory of urbanism in the information age, in B. Sanyal (ed.), Comparative Planning Cultures, London: Routledge, pp. 45-63.

Crang, M. and S. Graham (2007) Sentient cities: Ambient intelligence and the politics of urban space, Information, Communication \& Society, 10(6): 789-817.

Cresswell, T. (2006) On the Move: Mobility in the Modern Western World, London: Routledge.

Elliott, A. and J. Urry (2010) Mobile Lives, Abingdon: Routledge.

Farias, I. and T. Bender (eds.) (2010) Urban Assemblages. How Actor-Network Theory Changes Urban Studies, Abingdon: Routledge. 
Farman, J. (2012) Mobile Interface Theory: Embodied Space and Locative Media, Abingdon: Routledge.

Gordon, E. and A. de Souza e Silva (2011) Net Locality: Why Location Matters in a Networked World, Chichester: Wiley-Blackwell.

Graham, S. (2010a) When infrastructures fail, in S. Graham (ed.), Disrupted Cities: When Infrastructure Fails, Abingdon: Routledge, pp. 1-26.

Graham, S. (2010b) Cities Under Siege: The New Military Urbanism, London: Verso.

Hajer, M. and A. Reijndorp (2001) In Search of New Public Domain, Rotterdam: Nai Publishers.

Harder, H., N. Akkelie, A. S. Jensen, K. H. Reinau, and M. Weber (2012) Time use and movement behaviour of young people in cities: The application of GPS tracking in tracing movement pattern of young people for a week in Aalborg, paper presented at Space Syntax Symposium 8, SSS8, Santiago de Chile at the Pontificia Universidad Católica de Chile, Chile, January 3-6.

Huang, W. (2012) ICT-oriented urban planning strategies: A case study of Taipei city, Taiwan, Journal of Urban Technology, 19(3): 41-61.

Jensen, O. B. (2006) Facework, flow and the city: Simmel, Goffman and mobility in the contemporary city, Mobilities, 2(2): 143-165.

Jensen, O. B. (2007) City of layers. Bangkok's sky train and how it works in socially segregating mobility patterns, Swiss Journal of Sociology, 33(3): 387-405.

Jensen, O. B. (2009) Flows of meaning, cultures of movement: Urban mobility as meaningful everyday life practice, Mobilities, 4(1): 139-158.

Jensen, O. B. (2013) Staging Mobilities, London: Routledge.

Jensen, O. B. and B. S. Thomsen (2008) Performative urban environments: Increasing media connectivity, in F. Eckardt, J. Geelhaar, and L. Colini (eds.), Mediacity: Situations, Practices and Encounters, Berlin: Frank \& Timme, pp. 407-429.

Jørgensen, K. M. (2011) Nye medier-nye steder, Byplannyt, 4: 18-19.

Kitchin, R. (2011) The programmable city, Environment and Planning B: Planning and Design, 38(6): 945-951.

Kitchin, R. and M. Dodge (2011) Code/Space: Software and Everyday Life, Cambridge, MA: MIT Press.

Knudsen, A.-M. S., H. Harder, A. K. Simonsen, and T. K. Stigsen (2011) Employing smart phones as a planning tool: The Vollsmose case, paper for the 4th Nordic Geographers Meeting, Roskilde, May 24-27.

Marling, G., O. B. Jensen, and H. Kiib (2009) The experience city: Planning of hybrid cultural projects, European Planning Studies, 17(6): 863-885.

McCullough, M. (2004) Digital Ground. Architecture, Pervasive Computing, and Environmental Knowing, Cambridge, MA: MIT Press.

Moeslund, T. B. (2012) Introduction to Video and Image Processing: Building Real Systems and Applications, Berlin: Springer.

Nold, C., O. B. Jensen, and H. Harder (2008) Mapping the City: Reflections on Urban Mapping From GPS to Community Dialogue, Aalborg: Department of Architecture and Design, Department Working Paper Series No. 25, December.

Poulsen, E. S., H. J. Andersen, and O. B. Jensen (2012a) Full scale experiment with interactive urban lighting, paper for the Interactive Lighting Workshop. Newcastle, UK, June.

Poulsen, E. S., H. J. Andersen, O. B. Jensen, and M. Brath (2012b) Mediated urban architecture: Red Pavilion, in V. Andrade, S. Smith, and D. B. Lanng (eds.), Musings: An Urban Design Anthology, Aalborg: Aalborg University Press, pp. 36-45. 
Reinau, K., H. Harder, and A. S. Jensen (2012) The use of GPS and SMS data in illuminating the mobility of families: Fusing quantitative and qualitative data, paper presented at the Association of American Geographers Annual Meeting, New York, February $24-28$.

Sheller, M. and Urry, J. (eds.) (2006) Mobile Technologies of the City, London: Routledge.

Shepard, M. (ed.) (2011) Sentient City: Ubiquitous Computing, Architecture, and the Future of Urban Space, Cambridge, MA: MIT Press.

Simmel, G. (1909) Bridge and door, in N. Leach (ed.) (1997) Rethinking Architecture. A Reader in Cultural Theory, London: Routledge, pp. 66-69.

Suenson, V. (2012) Konstruktioner \& aktiviteter: En RFID undersøgelse af sociale aktiviteter i danske kulturhuse [Constructions and activities: An RFID investigation of social activities in Danish culture houses], Aalborg: Department of Architecture, Design and Media Technology, $\mathrm{PhD}$ thesis.

Suenson, V. and H. Harder (2011) RFiD tracking af brugeres adfærd i bygningsrum [RFID tracking of user behavior in buildings], Geoforum Perspektiv, 19: 29-38.

Tranberg, S., M. Svenstrup, H. J. Andersen, T. Bak, and O. B. Jensen (2009) The SantaBot experiment: A pilot study of human-robot interaction, Proceedings of the 4th ACM/IEEE International Conference on Human Robot Interaction. ACM Conference on Computer-Human Interaction, La Jolla, California, March 11-13, pp. 211-212.

Urry, J. (2007) Mobilities, Cambridge: Polity.

Vannini, P., L. Budd, O. B. Jensen, C. Fisker, and P. Jirón (eds.) (2012) Technologies of Mobility in the Americas, New York, NY: Peter Lang.

Wilken, R. and G. Goggin (eds.) (2012) Mobile Technology and Place, Abingdon: Routledge.

\section{Websites}

Centre for Mobilities and Urban Studies (C-MUS) http://c-mus.aau.dk/.

Research Cluster for Mobility and Tracking Technologies (MoTT) www.create.aau.dk/ $\mathrm{MOTT} /$. 
This page intentionally left blank 
Epilogue 
This page intentionally left blank 


\title{
Chapter 14
}

\section{Restless}

\section{Locative media as generative displacement}

\author{
Teri Rueb
}

My work begins with an invitation. Take a walk or a ride. Get out and explore the everyday environment in a new way, using the movement of the body as a means of releasing the mind, allowing it to wander - and to wonder, critically and aesthetically.

In these works participants explore a site while carrying a portable computer or cell phone equipped with a global positioning satellite (GPS) receiver. The GPS tracks the participant's movement and the resulting data is used to activate sound playback in response to their changing position in the landscape. Each work is a composition of geolocated sound regions specified in software that runs locally on the device, coupling virtual and physical space at the scale of landscape.

Participants literally bring the work into being through the physical action of walking, bicycling, driving, etc. Through kinesthetic engagement, body and environment come into contact as if in a dance composed of everyday movements. The sound overlay in each work brings attention to the physical and social contours of the natural and built environment even as it challenges participants to unconventional habitations - a kind of reading against the grain of the physical text and context of the environment. Through the sound overlay I seek to tease out, highlight, and choreograph a sampling of physical and social elements, itineraries and events that are inherent to a site. However, the primary structure of the work emerges from the unpredictable actions of the participant herself who performs in dialogue with variable social and environmental conditions. This interaction of site and subject, where each emerges through the confluence of physical, social, cultural, and technological forces acting upon each other in situ, is a defining aspect of "locative media."

The term "locative media," coined by Karlis Kalnins (Galloway and Ward 2006), emerged around 2002 as a way of distinguishing cultural uses of mobile media which critiqued the notion of "space" as an a priori or absolute abstraction and reinscribed "place" as a culturally specific and historically grounded concept. In this chapter I use the term "mobile experience" to refer to the broad domain of everyday experience that is mediated by location-sensing technologies, including commercial and industrial productions. "Mobile experience" 
encompasses the full spectrum of technologies that wed physical and virtual spaces to each other geospatially via software, regardless of their claim to the more specific designations of "locative media." Mobile experience involves the use of mobile media which engender a shifting of the sensorium that emerges as a result of our habitual use of these technologies in everyday life. Marshall McLuhan (1964) is credited with first observing that our interactions with media, as extensions of the body, have the effect of altering the perceptions and sensitivities of the human sensorium. For example, one effect of this shifting sensorium in response to the proliferation of mobile media is a heightened awareness of the reception quality of GPS, WiFi, and cellular networks (and their combination) as they relate to our perception of the built environment and our movement within it. Hence, mobile experience, not exclusive to the domain of locative media, is composed of a constant flux of physical, cultural, and psychological displacements where the hybrid physical and virtual contexts in which we increasingly interact create a third space, or what Sabine Breitsameter (2003) has called a hybrid space. This hybrid space can be disorienting, destabilizing, and decentering of the body as well as our sense of place and cultural identity. Rather than seeing these frictions as a negative effect of mobile media-something to be "designed away" or mitigated, I argue that acknowledging and embracing such instabilities actually forces us to productively negotiate what are alwaysalready shifting dimensions of a hybrid spatial condition vis-à-vis our perception and experience of place, and thus cultural identity. This constant process of negotiation underscores the inherently unstable condition of subjectivity which, while a given in all cultural contexts, may be exercised as a critical design agenda in highly technologized, mobile, and multicultural societies.

Just as an increasingly technologized and global society entails increasingly complex collisions of cultures and identities, mobile experience compounds these effects not just at the level of the physical sensorium, but also at the level of cultural identity and subjectivity. Technologies, bodies, and subjectivities are inseparably intertwined in everyday experience, and mobile technologies further intensify these entanglements. Mobile interfaces couple bodies in motion with places in motion - as process - blurring local with global, public with private, physical with virtual, and the proverbial "here-and-now" with "there-and-then." In this way they contribute to and intensify the constant displacement of bodies, sites, and subjectivities in highly mobile technologized society.

Inherent to the condition of mobile subjectivity, such displacements frustrate constructions of place, subjectivity, or the body as stable or fixed entities. This instability extends to authorship, too, which becomes a shared act in locative media works, not just at the level of interpretation, but in the very physical process of bringing the work into being. Authorship, like meaning, becomes emergent, contextual, and kinesthetically inflected, especially in locative media works that tend to exploit the indeterminate conditions of moving bodies in hybrid spaces. In all mobile experience, whether acknowledged or not, displacements of bodies and meanings unfold like constantly shifting horizons of 
context, meaning, and interpretation. In locative media such displacements are embraced and indeed emerge as unique qualities of this new form, medium, and genre. Through elaborating these effects as they generate and complicate meaning in artworks taken from my own practice, I present a position from which locative media may be understood as holding the potential for a kind of generative displacement.

This chapter presents a practice-based conceptual framework for imagining locative media as a form of generative displacement. Weaving concepts drawn from process philosophy and affect theory through a narrative of my own locative media practice as it has evolved over the past fifteen years, I will aim to reveal locative media as a form of generative displacement where the body is reconfigured in its relation to itself (the sensorium), to the environment (through both physical and cultural perception of place), and to others, including human and non-human realms. Through Elizabeth Grosz's (2008) reading of Deleuze and Guattari (1988) I will trace the ways in which emergence, embodiment, and the affective come together in the experience of the sensory-sensual body ${ }^{1}$ as it moves through and produces variously politically and culturally charged landscapes. Four projects that engage collisions of bodies and landscapes will be addressed: the shifting sensorium itself as a kind of landscape (Drift 2004); the affective experience of post-industrial waste landscapes (Core Sample 2007); perception and representation of landscape and cultural identity in globalized media culture (Elsewhere: Anderswo 2009); and the contested meanings of place and identity in post-colonial discourses about "wilderness" as it relates to landscapes of the Southwestern United States (No Places With Names 2012).

\section{A landscape approach to locative media}

Having made interactive public art installations in outdoor settings since 1990, I draw upon landscape as a first framework for understanding mobile experience. Landscape and the body are mutually constituted in mobile experience. A continuing point of reference for this is found in the history of sculpture, performance, and installation, all of which engage the body in spatial interactions writ large at the scale of landscape. The performative role of the participant is essential to the production of meaning in mobile experience. Minimalism recognized this, especially in the context of large-scale outdoor sculpture and land art, drawing attention to the phenomenological experience of the viewer as performer/participant. In particular, Robert Smithson (1979) articulates this aspect of his work as the critical unfolding of view upon view through the actions of a walking participant-observer who perambulates around the space of the sculpture, which itself functions as a kind of landscape. Smithson was among the first artists to create earthworks such as Spiral Jetty (1970), an enormous raised earth form shaped like a spiral that extends into the Great Salt Lake in Utah. He argues that the kinesthetic mode of consumption required in experiencing this work from the ground is essential to comprehending, understanding, and appreciating 
it. The scale of the work in relation to the body distinguishes it from traditional sculpture which was conventionally presented on a pedestal in a gallery or as a bounded and contained object in outdoor settings, viewable within the scope of a singular static sweep of the gaze. Earthworks such as Smithson's can be seen as providing an historical precedent and theoretical lineage to locative media especially as the performer/participant is framed as a kinesthetic agent in the construction of the work and its meaning.

Yves Alain Bois (1984) presents this argument succinctly, comparing Robert Smithson's (1979) and Richard Serra's (1982) writings about their early land art and earthworks in his essay "A Picturesque Stroll Around Clara-Clara." (Bois 1984) Richard Serra, best known for his enormous steel sculptures, is quoted. Speaking of his work Rotary Arc (1980), Serra writes: "The site is redefined, not represented ... the placement of all structural elements in the open field draws the viewer's attention to the topography of the landscape as the landscape is walked" (quoted in Bois 1984: 34).

Echoing this emphasis on the walking viewer, Smithson (1979) relates Serra's (1982) work to the notion of the picturesque, which he defines in relation to Heidegger's notion of a "thing-for-us:"

The picturesque, far from being an inner movement of the mind, is based on real land; it precedes the mind in its material external existence. We cannot take a one-sided view of the landscape within this dialectic. A park can no longer be seen as a "thing-in-itself," but rather as a process of ongoing relationships existing in a physical region — the park becomes a "thing-for-us."

(quoted in Bois 1984: 36)

In each case the emphasis is on the actual land and the experience and perceptions of the walker/viewer as she traverses it. In mobile experience site functions in a similar manner as a "thing-for-us," yet perhaps even more so as the site of mobile experience is a flux of physical and virtual spaces and times that are experienced both individually and collectively as unique itineraries through matter and media.

Through my work I critically reconfigure relationships of place and subjectivity through drawing attention to entanglements of context, movement, and perception as processes through which places, subjectivities, and identities emerge. My practice participates in these entanglements, seeking to expose or bring to critical consciousness their various permutations and cultural effects, including the ways in which space, place, and cultural identity are ineluctably bound to each other in mobile experience.

\section{The word itself}

The concept of landscape complicates distinctions between the proximal and distal, urban and rural, developed and undeveloped, local and global, the human 
and non-human, nature and culture, thing and process. Thus it provides an especially useful conceptual pivot around which very different ways of knowing and doing may come together and be used to reveal the operations of mobile network culture and society (Castells 2010).

The very word landscape has multiple, and often contested, meanings. It productively opens on to an expansive and inclusive field of inquiry and practice around questions of space, place, environment, and subjectivity. Any attempt to locate the meaning of the word reveals not only its extraordinary polyvalence, but also the reflexivity of landscape itself as a contextualizing function. Depending on how it is defined, landscape may be understood to provide the backdrop for the environment in which humans dwell, or as the environment itself. Whether understood as biophysical, perceptual, cultural, or political, it indicates a set of relationships between humans and environments, a play of human and non-human forces that ultimately suggests a process approach to the design space of mobility. Indeed, the very notion of what this "space" implies is of concern here as this paper seeks to assert a critical design agenda. The normative design space of mobile media is often limited to the screen of the mobile device itself, as information overlays are registered against maps or other interfaces reflecting feedback from real-time location tracking. In suggesting a "process" approach to the design of mobile media, I aim to shift the extent of this design space to explicitly include the whole space of body-site-movement interaction, not just on-screen interaction, with location-sensitive information or responses.

Across languages, cultures, and disciplines, landscape has strikingly different meanings, or may resist translation entirely. For example, landscape has a long history in the West of being associated primarily with the specular or objectifying gaze and the picturesque, in the sense of the pictorial. It has been objectified as resource for exploitation, object of the patriarchal or colonial gaze, and framed as feminized "nature" when treated as the passive "background" for human activity, rather than as active social, cultural, or material process (Cosgrove 1984). Throughout these varying contexts, however, the concept of landscape both explicitly and implicitly serves to frame and ground our understandings of place, culture, and identity. In this it performs as an active and contextualizing function that, like the physical form of landscape itself, is fundamentally temporal and constantly in flux. For example, in the American West, landscape is less operative as a cultural idea associated with a picturesque thing "out there" to behold. Instead, it is more commonly understood as "land," as in the materiality of the land as resource, as territory, as property, as physical obstacle or threat, and indeed, more holistically as climate, weather, and environmental condition. In such environments scale relationships alone, immediately evident to the casual observer, register and precisely locate the proper proportion and place of human and natural processes. ${ }^{2}$

As a means of suggesting that landscape might be understood more as process than artifact we might look to the medieval Germanic term Landschaft. In contrast to the pictorial or visual emphasis of the sixteenth-century Dutch term 
landskip, landschaft suggests an active tense in its connotation of social process. J. B. Jackson (1984: 7), the historian of vernacular landscape, suggests that the component "land" once signified "a defined space, one with boundaries, but not necessarily one with fences or walls." "Schaft" or "scape," he writes, "is essentially the same as shape, except that it once meant a composition of similar objects, as when we speak of a fellowship or membership ... the word scape could also indicate something like an organization or system" (1984: 7). As landscape itself is inherently temporal, unfolding with and over time, it can be seen as constituting a social process, rather than as social product. The Germanic echoes held within this concept of "landscape" offer a model for thinking landscape as more verb than noun.

Yet the image of landscape as an organization or system evokes a structuralist notion of landscape as artifact that can be understood through rational analysis of its various knowable parts. Thus, ultimately a more temporal image is necessary to account for a landscape approach to mobile media-something more fluid and process-oriented that suggests a becoming or unfolding, rather than a visual image (including the conventional notion of the picturesque, or the Dutch landskip or landskap) or fixed ontological entity expressed as a relatively stable social-spatial system (the positivist school of landscape that trace their foundations to the Germanic landschaft). In contrast to architecture, for example, or the association of landscape with cartographic imagery, landscape is first and foremost the expression of biological and geologic process. Thus, landscape must be understood as fundamentally defined by its biological form as physical processes unfolding in time. Complexity theory provides the basic reference for this emergent view of landscape. If we understand landscape as the complex interaction of subjects, technologies, and spaces produced through biological, social, neural, physical, and anthropological processes, then we suddenly find ourselves moving beyond the ocularcentrism of an art historical view or the historically positivist or structuralist reductions of social science. Mobile media, too, as I have argued above, are inherently more temporal and fluid than media environments tied to fixed or moving images on ubiquitous screens - media contexts that are explicitly designed to be used everywhere and nowhere.

A process approach to landscape and mobile media can accommodate the heterogeneous scalar, temporal, material, biological, social, and cultural qualities that are suggested by the mobilities and subjectivities produced through and with mobile media. In this model, landscape as process can be seen as the ground from which mobile experience emerges as "becomings" of heterogeneous sites, subjectivities, and materialities. Landscape and mobile experience are thus ultimately performative in the sense of action and participation of the subject, as well as biological and social processes.

Yet for all its rich potential, a landscape approach to mobile media must take an even more radical leap in order to embrace the fullness of a temporal and process-based model. The cultural term locative media or industry labels "location-aware" or "location-based" designs and services still suggest a 
location or position-bound model of space haunted by a latent Cartesianism. Other models of spatiality that acknowledge the shifting relations of bodies, sites, and subjectivities must be called upon in order to effect this conceptual leap. Elsewhere I have articulated a similar "space-time" of sound installation and mobile media (Rueb 2002; Rueb and Breitsameter 2004) however, in developing this notion more critically I have looked to Deleuze and Guattari (1988).

The writings of Deleuze and Guattari (1988), with their emphasis on a temporally and materially articulated spatiality, lend themselves to imagining a project of "becoming" landscape. The notion of haecceity, in particular, evokes this conception of space:

In ["A Thousand Plateaus"], a body taken as a haecceity is defined in its cartography by its longitude (the "speeds and slowness" of its material flows) and by its latitude (its set of affects) (260-261). An environmental assemblage, a "set of relations" defined as a haecceity (382), treats spatiotemporal relations not as predicates of a thing (Aristotle's categories include "where" and "when") but as dimensions of multiplicities, components of the assemblage (262).

(Bonta and Protevi 2004: 94)

In addition to the notion of haecceity, the by now widely familiar concepts of "smooth" and "striated" space developed by Deleuze and Guattari (1988) offer a further image for thinking mobile media and space according to a temporal model. As just one example, the notion of smooth space as it pertains to the musical model implies a constant motion where there is continuous variation and continuous development of form, as opposed to striated space, which develops linearly and produces order and a succession of distinct forms (Bonta and Protevi 2004: 41).

Additionally, drawing upon the concept of "becoming-animal" or "becomingwoman" as developed in A Thousand Plateaus, we might imagine a "becominglandscape" where landscape ceases to be "other" in a binary opposition and is instead seen as an entanglement of various forces, human and non-human, that are constantly in process of becoming one another, rather than existing as ontologically stable categories. Mobile media, and the mobilities they engender, make legible such spatialities and temporalities. Far from the romantic notion of landscape as a sweeping view, or the positivist or structuralist reduction of landscape to territory or system, the image of landscape as spatial-temporal process reveals mobile experience and post-human mobilities as complex entanglements of bodies, materialities, temporalities, and subjectivities. Viewed from this perspective, locative media may be seen to challenge the totalizing view of GPS or surveillance technologies as the solely evil apparatus of control societies that employ them as tools of oppression in the regulation of power and knowledge via language, code, and prosthetic agency. 


\section{Displacements}

Places have agency; they work upon us as we work upon them. Discovering this agency and engaging it through shaping lively interactions between bodies and environments is fundamental to my practice. The feedback loops created in these exchanges effect displacements of various kinds. In thinking about how these displacements function at the level of mobility and perception, Merleau-Ponty's (2002) phenomenology emerges as a useful theoretical framework. In this model the subject is seen as inextricably linked to environment as affect, climate, attitude, or mood. Emphasis on the kinesthetic foregrounds movement as fundamental to perception, including the smallest and most subtle movements of the body including the tiny shiftings of the gaze, the rise and fall of the breath, and the subtle response to vibrations felt as touch, sound, and other sensations. In locative media body, mind, and environment are entangled in the circumambient sphere of embodied kinesthetic perception, as well as the physic-sensory realm. Here we might look to the work of Elizabeth Grosz ${ }^{4}$ (2008) whose discussion of landscape places it squarely at the confluence of phenomenology and process philosophy, especially as articulated in the work of Straus as compared to Deleuze and Guattari (1988) — who by the time they published A Thousand Plateaus had departed significantly from the lineage of phenomenology and its emphasis on the subject of lived experience versus the neuro-physiological subject:

Straus illustrates the distinction between perception and sensation in terms of the opposition between geography and landscape. Geography is the space of the map, that which is regulated by measurable coordinates, what Deleuze and Guattari refer to as striated or sedentary space, a space whose location or region is abstracted from its lived qualities. Landscape, by contrast is that space revealed by sensation, which has no fixed coordinates but transforms and moves as a body passes through it.

(Grosz 2008: 72)

In each of my works extensive interaction with sites unfolds through inviting participants to engage the simple act of walking. Both in making my work and experiencing it, walking is the ground from which each piece emerges. Through walking, responsive sounds, both ambient and composed, are coupled to the proprioceptive sensation of the participant's body in motion, giving rise to emergent invisible acoustic architectures that bind body and environment in a fluid feedback. Since the beginning I have used sound as the primary media overlay in my works, eschewing the screen in the interest of drawing the eye to the surrounding environment, as well as allowing the body to freely explore this novel spatiality.

I am intrigued by the aesthetic and kinesthetic qualities of GPS as a spatial medium and its capacity to enhance and intensify the coupling of movement and meaning in site-specific works. Through sculpting sound to site, as if it were a 
garment made to fit the landscape, these works unfold as a series of shifting thresholds that blur physical and virtual, organism and environment, body and technology, sound, sight, and touch. Through slowing down and becoming sensitive to thresholds of site and signal as structured through spatialized sound, I hope that participants may begin to feel a reconfigured relationship of the body in space as mediated by mobile technologies. Ultimately my work is an inquiry into the transformation of perception, cognition, and consciousness as technologies reshape our sense of place, identity, and embodied interaction. Cultural identity then, becomes a central question as these couplings and entanglements become evident at the level of representation and mobile experience. Toward elaborating these colliding forces, I use examples from my own works created over the past decade.

\section{The work itself}

Forms and modes of displacement engaged in my practice express differently in each work, but all of them bring the body into tightly coupled dialogue with the physical contours of a site, as well as the social, cultural, and historic continuities and contradictions inherent to sites as processes in time.

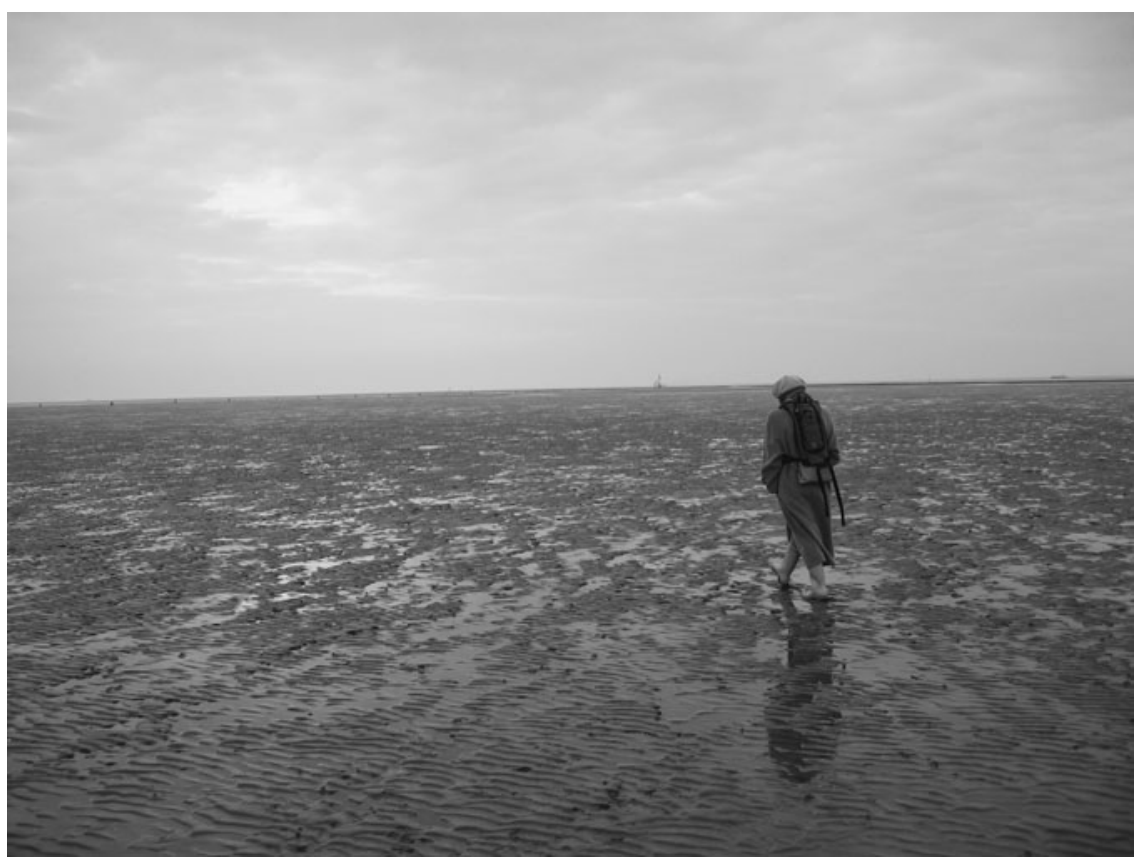

Figure 14.I Teri Rueb, Drift, 2004, Walker on the tidal flats. Photo courtesy of the artist. 
Drift (2004) is a site-specific responsive sound environment set along the tidal flats of the Wadden Sea in Northern Germany. Visitors are provided with headphones connected to small computers equipped with GPS and custom movement tracking software. As they explore the environment of the tidal flats, sounds play in response to their movement through regions of sound that drift with the tides. Absolute position and relative movement are explored as visitors discover they may experience this work by simply standing still, allowing the drifting sound regions to "wash" over them in time.

With Drift, displacement occurs in multiple domains. Sound regions are displaced with the tides, the body becomes displaced in an isomorphic landscape of shifting sand, water, wind, and signal. Awareness of nineteenth-century navigation techniques (line of sight landmarks, sonic beacons, etc.) blends with the direct engagement and encounter with contemporary technologies including radar, GPS, and GIS. Despite the presence and ubiquity of these artifacts of orientation and navigation, however, displacements of spatial awareness and general orientation in an interactive system occur as participants wander through a seascape unaided by the grounding visual and textual cues of locational technologies such as maps and GPS coordinate output. This short-circuiting of participants' expectations when using locative media frustrates goal-oriented or efficiency-driven modes of navigation and interaction.

As location-aware technologies and conventional modes of geospatial representation (e.g., Google Earth, GIS, etc.) became more commonly available in the early 2000s, they began to reshape everyday methods and modes of navigation and related spatial perceptions. Drift was an attempt to call our attention to these cultural transformations through direct physical engagement with the media in a mode of interaction that ran "against the grain" of these same popular media forms and navigation technologies.

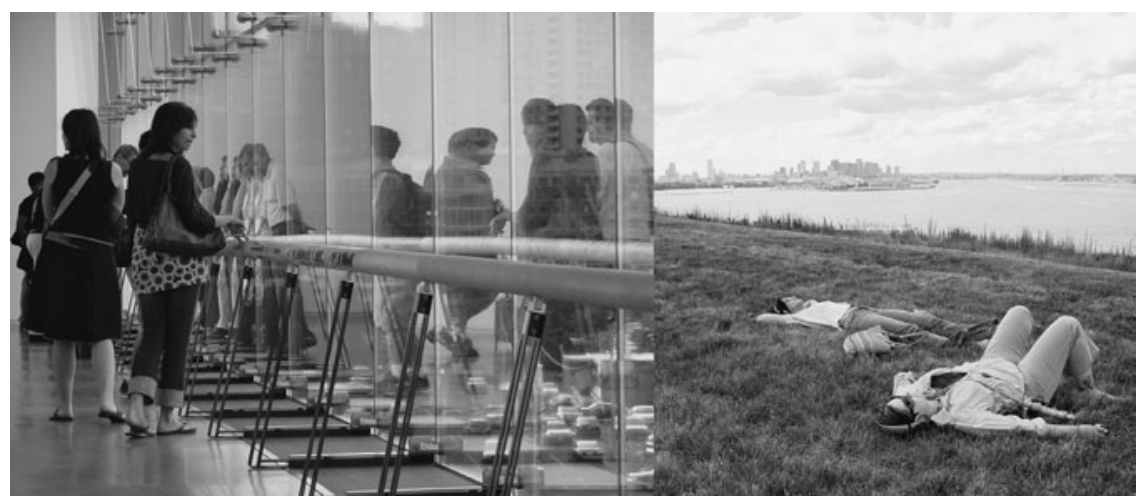

Figure 14.2 Teri Rueb, Core Sample, 2007, Visitors in the gallery (left), walkers on the island (right). Photo courtesy of the artist. 
With Core Sample (2007) I sought to evoke another kind of displacement. On one level, the work was intended to evoke an imaginary displacement of the body through the layers of a metaphoric core sample corresponding to the material history of the site. On another level, the site itself - an island long used as a dump by the city of Boston, Massachusetts - became the symbol of a displacement in the form of the abject or suppressed. As a marginal space, or waste landscape, Spectacle Island was long reviled as an eyesore known for its putrid smell, and toxic emissions of methane and leachate.

The project was born of the question, "How can we come to terms with our own role and place within processes of consumption, waste, and eventually reclamation, not through endless elaboration of the problem, but instead through an experiential encounter in which we feel both physically and symbolically implicated in and empathic to the very ground beneath our feet?" Formally I wondered, "What kinds of relationships to landscape might emerge if one could sink through the earth and become the layers of sediment that make up this reclaimed landfill?"

I attempted to create this effect through presenting participants with a layered sound composition that changed as they moved across the shifting elevations of an island, suggesting a metaphoric core sample. Spectacle Island is in large part made up of garbage - over thirty-meter cliffs of garbage in some places - as well as excavation from the Big Dig tunnel project that in some places reaches thicknesses of up to eighteen meters. The island used to be an open landfill where spontaneous methane fires burned out of control, lighting up the night sky.

The island now stands as a showcase for promising and progressive techniques of waste management and landscape remediation, cloaked in the image of a pastoral plain. An entirely engineered landscape, Spectacle Island was capped with excavation and completely covered with a scientifically formulated loam designed to support over 28,000 imported plants, trees, and shrubs that make up its current park-like facade.

Core Sample sought to reveal these radical physical, cultural, and psychological displacements, as well as evoke more subtle displacements as participants wandered the island and were immersed in sounds that evoked these materialities as well as the island's cultural history and possible futures. Sounds of garbage, leachate, escaping gases, and radio astronomy transmissions mix with the voices of past residents of the island who describe their experiences growing up on this island or working in its many industries. Over 250 sounds were combined with the voices of landfill technology experts and botanists who study the vibrant and remediating potentials of the botany of disturbed landscapes. Each sound was carefully composed and located in space such that specific elevations and transitions between elevations expressed a sonic stratigraphy of cultural, social, and material history as registered against a metaphoric core sample. In this way the project sought not only to displace participants' sensory and perceptual experience of the island as a material site, but also to displace them in the multiple temporalities and subjectivities suggested by this incredibly lively and resilient landscape. 


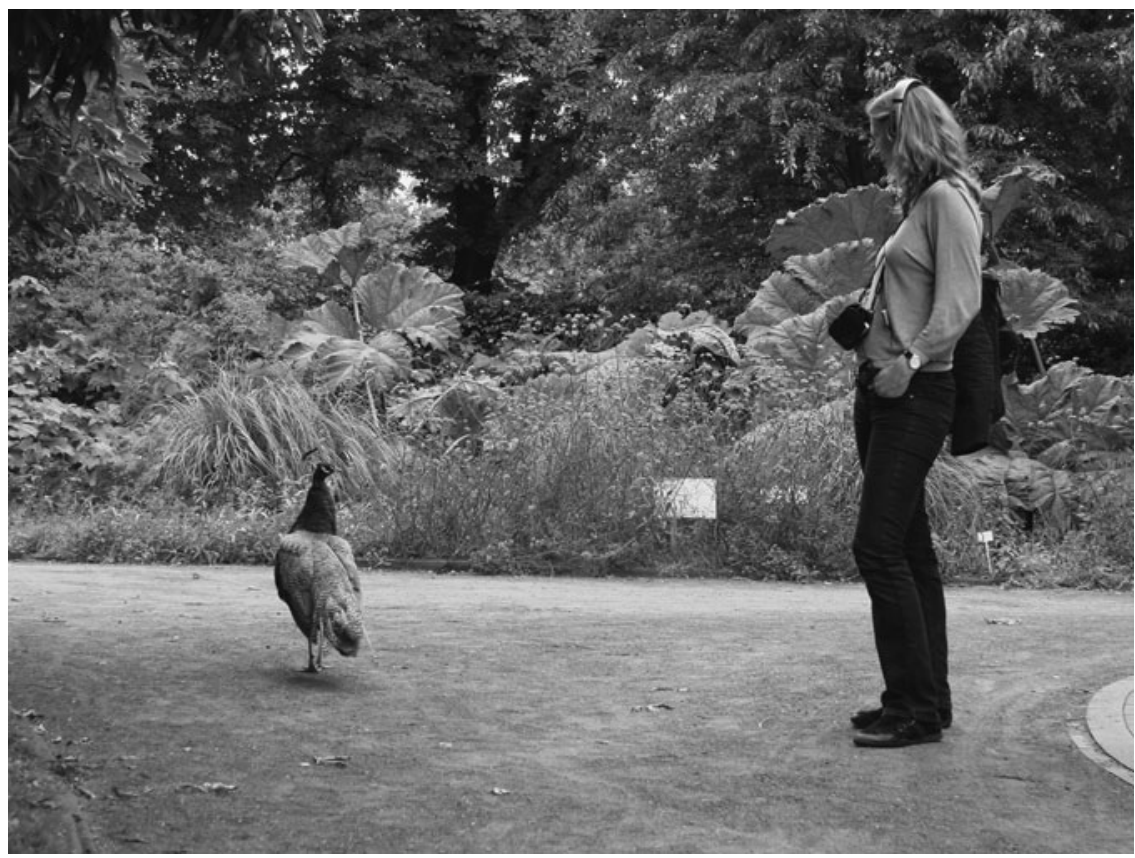

Figure 14.3 Teri Rueb, Elsewhere: Anderswo, 2009, Walker in the botanical garden. Photo courtesy of the artist.

Elsewhere: Anderswo (2009) brings the theme of displacement to the fore through embracing the cultural disorientations and disjunctions that are often felt when traveling to foreign places that confront us with the uncanny experience of being "outsider" to local custom and context. Set across two sites, the project engaged local displacements, as well, as viewers might hear similar soundsboth ambient and recorded-repeated across the two locations. Separated by approximately 100 kilometers, the corresponding installations set in Oldenburg and Neuenkirchen function dialogically as sites punctuated with a patchwork of appropriated audio from popular films, radio, and television that evoke familiar landscape references to both German and American audiences.

The last work I will discuss is perhaps the most complex in terms of addressing themes of displacement. No Places With Names: A Critical Acoustic Archaeology (2012) brings the question of displacement in mobile media to the fore as it explores the concept of "wilderness" as its meaning shifts across cultural and historical contexts. The work was created in collaboration with local artists Larry Phan (first generation Vietnamese-American) and Carmelita Topaha (Diné) and included interviews with over thirty-five local residents of the Santa Fe area in New Mexico including artists, farmers, and scientists, acequia majordomos, arts writers, and activists. Nearly a third of the interviewees were members of over a 


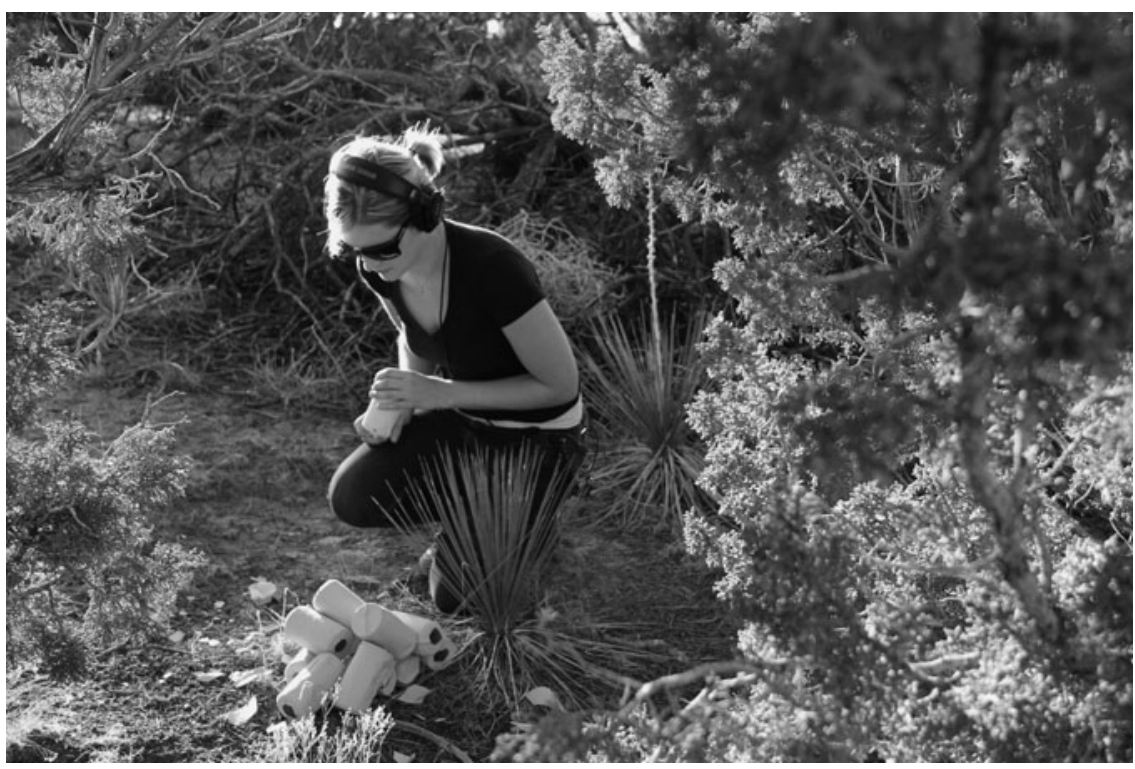

Figure 14.4 Teri Rueb and Larry Phan with contributions from Carmelita Topaha (Diné), No Places With Names, 20I2, Walker holding sculpture. Photo courtesy of Teri Rueb.

dozen different First Nations tribes from all over North America. The project itself was sited on the campus of the Institute of American Indian Arts, just south of Santa Fe, New Mexico.

The piece was developed over an eighteen-month period of residency in New Mexico and while it deeply engaged local sites and communities, the primary audience is often a tourist or short-term visitor to this highly trafficked travel destination, bringing another dimension of displacement and mobility to the work.

The question of wilderness in the cultural context of the Southwestern United States is first and foremost one of displacement. The Southwest is home to the densest population of contemporary Native Americans living on and off reservations and in this context they live alongside significant populations of descendants of Spanish colonial settlers dating back nearly 400 years. This mix is complemented by modern and contemporary waves of immigration to the area including members of the scientific elite in the 1940s and 1950s at Los Alamos Labs and counter-culture radicals of the 1960s and 1970s. This cultural milieu makes the Southwest an especially charged environment in which to raise questions regarding the notion of wilderness, an idea that has historically been invoked to promote religious, environmental, and colonial agendas since the earliest colonial period in the Americas. The idea of wilderness as the place of 
no people, a pristine refuge, or a natural resource that must be preserved are all reflections of contested ideologies that often had the impact of displacing people and natural habitats in the name of "progress."

Thus, to ask the question, "What does wilderness mean to you?" in these interviews and to present the fragmented narratives that emerged in response to this question in locations that have historically typified the cultural imaginary of "wilderness" effects an odd kind of double operation of displacement. In most cases interviewees responded that the word held little meaning as it was seen to create false binaries of nature and culture, or was seen to have deliberate connotations of erasing the 15,000 year history of indigenous habitation of the area prior to colonization. Some interviewees saw environmental groups and government agencies misuse the word in the interest of reappropriating ranch and farm land to "preserve" it as designated "wilderness" areas. These are only a few of many different responses that became the sonic fabric of the final work which was presented in situ as a site-specific sound walk on the campus of the Institute of American Indian Arts, itself a parcel with a long history of having been used for grazing, short-term habitation, and, more recently, suburban development, including the campus itself. Each of these historical moments was marked by displacements of native peoples, plants, and watersheds and ultimately the reclaiming of the site from cattle grazing uses to the reconsecration of it as the site of an institute dedicated to the advancement of native arts. Onto this palimpsest of spatial and social displacements the piece No Places With Names introduces yet another layer of displacement in inviting people, most of whom will be visitors or "outsiders" to the campus community, to walk the trails and reflect on the complex spatial and social ramifications of landscape imaginaries, including the virtual overlay of the mobile sound walk itself - an art form that while readily embraced by students and faculty, is still nevertheless considered a distinctly unconventional or alien art form.

In closing, it should be said that while I certainly remain an outsider to indigenous and Nuevo Mexicano cultures, it was clear to me that among the important differences in their world views regarding landscape was the sense that landscape is produced through cultural action and use in both cultures. For indigenous peoples this is intrinsically tied to their oral history, origin stories, and moral code. Like Australian aboriginal songlines, Native Americans have elaborate stories that are tied to landforms and itineraries connecting landforms. It is believed that actually visiting those sites reveals those stories and renews their power as catalysts in organizing human society and its balance with nature. Bruce Chatwin (1988), Keith Basso (1996) and Steven Feld (Feld and Basso 1997) have all written works that have illuminated these various cultures and their conceptions of space, place, and landscape from the perspective of situated ethnography. While their works have profoundly influenced my practice since 1996, I had an even more powerful encounter recently as I lived in New Mexico for a year and had the privilege of collaborating on a project with ceramicists Carmelita Topaha (Diné) and Larry Phan, a first generation VietnameseAmerican, both of whom lived in Farmington, New Mexico. This experience has 
left me both grateful for the deeper perspectives gained, and humble at the thought of speaking with any authority about these cultures which I now appreciate as even more complex and elusive to my understanding than I ever imagined. Nevertheless, I will try to evoke something of their significance to my argument.

Distinctions of nature and culture are much more blurry in the cosmology of Native American cultures, perhaps suggesting a model of "becoming-animal" or "becoming-landscape." For Nuevo Mexicanos landscape seems to be understood in relation to cultivation and sustenance. Rather than thinking of distinct categories of "wilderness" and "civilization," their world view sees the land as a resource that must be managed collectively as a framework for governance. In fact the acequia, or irrigation ditch systems, they brought to the new world from Spain define a unique social contract organized around a shared resource. To this day, the acequia "majordomo" holds an important role in overseeing the relationship between people, resources, and the law. The majordomo is much more than a symbolic leader. He or she is tied to the land and to the watershed as a manifestation of another order, that of nature and the precious resources provided by nature to sustain a people. In the harsh desert climate of the Southwest, water is everything. It defines the relationship between people and the environment at the most profound level. Take for example the fact that Hopi religion is so intrinsically defined by the need to supplicate the spirits or "kachinas" in the interest of calling forth rain to sustain crops that even artesian water on Hopi land is not drawn upon as a significant resource to water crops - it being water from the earth instead of the sky. These ruminations are certainly limited in their understanding of Native American and Nuevo Mexicano ways, but still I find in them the promise of a fruitful way to think landscape newly as a "becoming"even a "becoming-environment," if you will.

\section{Conclusion}

Much more might be said about the various displacements effected by this final work, but in conclusion I would like to invoke the overarching displacements that have become apparent to me over the past fifteen years of creating works in locative media. There is a reductionist function of GPS and location-sensing technologies and interfaces that would represent us as tiny dots moving about the flattened plane of an abstract projection of three-dimensional space. In our entanglements with these technologies we are simultaneously in a first and third person subject position-registering our presence "here," but from a position outside our corporeal being.

Similarly, yet in a different historical moment, seeing the image of the Earth from outer space represented a kind of mirror stage in human consciousness akin to the emergence and proliferation of Google Earth and Google Maps as commonplace modes of representation and enaction of our daily "here-ness" and mobility. I often wonder what the equivalent auditory moment is to the visual 
mirror stage described by Lacan. What would a sonic "mirror stage" be that precedes the visual as we hear acutely even when we are still in the womb, our eyes sealed? How does this sonic sense of proprioception relate to our proprioceptive awareness of here-ness as thrown back on us in a double first person/third person coordinate reference translated as dot on a Cartesian map, the cognitive awareness that haunts nearly every moment of our interface with location-sensing technologies? Perhaps more disturbing, especially as it lingers even deeper in our subconscious awareness, is the fact that this image holds within it the sense of a self moving in relation to the planet(s) - a movement that is sensed by satellites that are themselves moving in relation to the planet(s). The scale and significance seems nothing short of the intimate immensity that Blake speaks of when he describes seeing the world in a grain of sand, and holding infinity in the palm of your hand. A strange new "unhomeliness" seems to characterize the current moment of "i-" and "my-" everything, intensified by mobile technologies that suggest that anything we desire is magically at the tip of our fingers, under our thumbs, or already in the palm of our hands. But these new formulations are intrinsically complicated by the fact of their temporal scale-one in which constant fluctuations and movement force us to understand our existence as more motion than stasis, more fluctuation than constancy, more indeterminate than determinate. In this state of constant (dis)orientation, how can we find a reference point against which to define ourselves and the places that define us? I would argue that the very desire for such fixity is an unnecessary source of frustration. If we could find our peace in the current, the flow, the constant change, we might be released from the fiction of fixity which leads us to think of subjectivity as objective, unchanging, constant, and consistent - indeed this false image underlies the hegemonic logics that fuel racism, sexism, species-ism, and countless other instances of exploitation and a mistaken sense of superiority. Designing for a constantly shifting point of view that challenges the centrality of any singular subject position may lead us to embrace our mobile subjectivity as a form of productive displacement. A decentering that engenders landscapes of becoming in which radical subjectivities may be realized across human and nonhuman domains.

\section{Notes}

1 In The Production of Space, Henri Lefebvre (1991) describes the resistance of the "sensory-sensual" body to the abstraction that characterizes representational spaces, which include architectural drawings, monuments, mathematical descriptions, or systems of spatial representation, etc. In resistance to such spatial reductions, the sensory-sensual body exerts itself in the space of festivals, political demonstrations, and leisure.

2 This distinction between "landscape" and "land" as marking perceptions of East Coast versus Western cultures in the United States emerged in conversation with Lucy Lippard during an interview I conducted with her on May 25, 2012.

3 See Bonta and Protevi (2004) for a useful glossary of Deleuze and Guattari's terminology. 
4 I have drawn heavily upon Elizabeth Grosz's reading of Deleuze and Guattari in her 2008 work Chaos, Art, Territory: Deleuze and the Framing of the Earth because in it she interprets their ideas in architectural terms and with respect to art in a much more spatially oriented analysis than even Deleuze and Guattari, whose references to art tend toward music and painting. Furthermore, the reference to Straus is a unique linkage of phenomenology and process philosophy - a contribution that Grosz is making in drawing the two together around an analysis of "geography" versus "landscape." The integrity of her contribution is, I believe, maintained in quoting her, rather than reconstructing the analysis through a re-reading and recontextualizing of the original texts only to produce a juxtaposition that was initially made by Grosz herself.

\section{References}

Basso, K. (1996) Wisdom Sits in Places: Landscape and Language Among the Western Apache, Albuquerque, NM: University of New Mexico Press.

Bois, Y.-A. (1984) A picturesque stroll around "Clara-Clara," trans. J. Shepley, October, 29: $32-62$.

Bonta, M. and J. Protevi (2004) Deleuze and Geophilosophy: A Guide and Glossary, Edinburgh: Edinburgh University Press.

Breitsameter, S. (2003) Acoustic ecology and the new electroacoustic space of digital networks, Soundscape Journal of Acoustic Ecology, 4(2): 24-30.

Castells, M. (2010) The Rise of the Network Society: The Information Age: Economy, Society and Culture vol. 1 (second edition with a new preface), Chichester/Malden, MA: Wiley-Blackwell.

Chatwin, B. (1988) The Songlines, New York, NY: Penguin Books.

Cosgrove, D. (1984) Social Formation and Symbolic Landscape, London: Croom Helm.

Deleuze, G. and F. Guattari (1988) A Thousand Plateaus: Capitalism and Schizophrenia, London: Athlone Press.

Feld, S. and K. Basso (eds.) (1997) Senses of Place, Santa Fe, NM: School of American Research Press.

Galloway, A. and M. Ward (2006) Locative media as socialising and spatializing practice: Learning from archaeology, Leonardo Electronic Almanac, 14(3), available at: www.leoalmanac.org/wp-content/uploads/2012/07/Locative-Media-As-SocialisingAnd-Spatializing-Practice-Learning-From-Archaeology-Leonardo-ElectronicAlmanac.pdf.

Grosz, E. (2008) Chaos, Art, Territory: Deleuze and the Framing of the Earth, New York, NY: Columbia University Press.

Jackson, J. B. (1984) Discovering the Vernacular Landscape, New Haven, CT: Yale University Press.

Lefebvre, H. (1991) The Production of Space, London: Blackwell.

McLuhan, M. (1964) Understanding Media: The Extensions of Man, New York, NY: McGraw-Hill.

Merleau-Ponty, M. (2002) Phenomenology of Perception, London/New York, NY: Routledge.

Rueb, T. (2002) Sonic Space-Time: Sound Installation and Secondary Orality, Proceedings of the CAiiA Consciousness Re-Framed Conference, Perth, Australia.

Rueb, T. (2004) Drift, available at: www.terirueb.net/drift.

Rueb, T. (2007) Core Sample, available at: www.terirueb.net/core_sample. 
Rueb, T. (2009) Elsewhere: Anderswo, available at: www.terirueb.net/elsewhere.

Rueb, T. (2012) No Places With Names, available at: www.terirueb.net/place_names.

Rueb, T. and S. Breitsameter (2004) Sound art and Hertzian space: Teri Rueb in conversation with Sabine Breitsameter, available at: www.audiohyperspace.de/ 2004/page/4.

Serra, R. (1982) Notes from Site Point Road, Perspecta,19, Cambridge, MA: MIT Press.

Smithson, R. (1979) Frederick Law Olmsted and the dialectical landscape, in N. Holt (ed.), The Writings of Robert Smithson, New York, NY: New York University Press. 


\section{Index}

Page numbers in italics denote tables, those in bold denote figures.

a-mobile theories 67

action plans 175

activism 66

activity fragmentation 190

adjacent possibles 1-2

advertising revenue 151

agency 157

Alexander, C. 192

algorithms, behavior manipulation 10

Alternate Reality Games (ARGs) 135

alternative experiences 113

Altman, I. 85

Anker, N.E. 170

Anker, P. 170

app economy 136-9

Archigram 201-2

architectural competitions 211-12

architectural model making 211-12

Art+Communication Festival, Latvia 134

associative society, vs. communal 20-1

attention, management of 48

Audiotopie 126-7

Augé, M. 9, 124, 128, 129

augmented realities 208-13

augmented reality browsers 4

authorship 242

autotelic playfulness 34

Awan, N.T. 174

badges $94-5$

Baudrillard, J. 152-3

Beck, G. 75-6

Beck, U. 78

Beer, D. 156

behavior manipulation, algorithms 10

Belly 157
Beniger, J. 20-1

Berger, P. 21-2, 26

biometrics, for border control 65

Blake, W. 255-6

Blast Theory 136, 173

blogs 110

Boetzkes, A. 125

Bois, Y.A. 244

Bolish, M. 135

Bonta, M. 247

book: aims 13; background 5; origins 6 ; structure and overview 6-13; terminology 13

border control, biometrics 65

border crossing: implications 74 ; as performance art 72

Border Security, Economic Opportunity and Immigration Modernization Act (US) 78

borderization, vs. globalization $73-8$

borders: escalation of control 78; permeability 68 ; politics of $67-9$

Boulding, K. 26

boundaries, breaching 1-2

Bourdieu, P. 68

Breakout! project 200-1

Breitsameter, S. 242

bricolage 137

Brown, B. 91

Bucharest 174, 176

Bull, M. 122

Bunschoten, R. 173-6

buskers 125

camera glasses 50, 51-2

camera tracking $231-2$ 


\section{Camover 2013145}

Can You See Me Now? 154

Cardenas, M. 73

Cardiff, J. 127, 134

cars: as basis of mobility 27 ; choice to own 19-20; dual character 27-8; and mobile phones 28-9

Castells, M. 189, 245

Cattel, M.G. 112-13

Center for Oral History and Digital Storytelling (COHDS) 127

Centre for Mobilities and Urban Studies (C-MUS) 225, 226-7

chance, and playfulness 34

chance orchestration $7,34-5,39$; forms of 42-4; future research 44-5

check-ins 111; Foursquare 108-9

choice 19,40

CHORA 173-4

cities: information networks 103; mobile and locative technologies 12 ; ways of understanding 224

city transit 9-10; art and performance 124-5; communitas 119, 124-5; context and overview 117-18; as hybrid space 126-8; as liminal space 119-23, 128; Los Angeles 128-9; methodology 118-19; music 122; as performance space 123-4; pleasure of 117-18; rhythm 123; rush hour 123-4; summary and conclusions 128; Toronto 129

Climo, J.J. 112-13

cognitive switching 50

communal society, vs. associative $20-1$

communication, transport and coordination 27-9

communitas $119,124-5$

commuting see city transit

computer-aided design/computer-aided

manufacturing (CAD/CAM) 211

connectivity $7,188-9$

consumption see gamification

context, of mobile technology use 190-1

control: and industrialization 20-1;

location-aware technologies 65

coordination: of social life 34-5; transport and communication 27-9

Core Sample 251

Corner, J. 172, 175, 176, 183, 184

Cosgrove, D. 170

Cosmobilities Network 2

Covington, L. 93, 94
Cresswell, T. 66, 68, 73-4, 104

Critical Cartography 170

Crowley, Dennis 36

cultural identity 242

cultural mapping 11; context and overview 167-9; future developments 184; mobile mapping 172-6; summary and conclusions 184-5; urban mapping 169-72; see also Streetscape

Cunningham, C. 153

daily lives, documentation of 95-6

Dasein 127

data mining 10

de Certeau, M. 7, 33, 34-5, 39, 40-1, 117, 120

de Peuter, G. 144, 159

de Souza e Silva, A. 6, 33, 34, 72, 85, 108, $126,127,135,154,155-6,157,173$, 181,189

Deetz, S.A. 157

Delaney, D. 67

Deleuze, G. 247, 248

demographics 96

Denmark see ICT toolbox

detachment, from place 103

Detours: Poetics of the City (Ng-Chan) 127

dialogue, between disciplines 6

Digital Culture in Architecture (Picon) 202

digital enclosure 153

digital Gemeinschaft 21-2

digital turn, in architectural design 211-12

displacement: context and overview 241-3; Core Sample 250, 251; discussion and examples 248-55; Drift 249; Elsewhere: Anderswo 252, 252; landscape approach 243-7; No Places With Names: A Critical Acoustic Archaeology 252-5, 253; summary and conclusions 255-6

distance, perceptions of 101

documentation, of daily lives 95-6

Dodge, M. 226

domestic space, gamification 157-8

Dominguez, R. 69, 71, 75, 77

domotics 159

double data bodies $72-3$

Drakopolou, S. 134

Drift 249-50, 249

driving see mobile multi-activity 
Dumas, C. 125

Dunn, N. 211

Durkheim, E. 24-5, 120

Dyer-Witheford, N. 144, 159

economy of attention 62

Edensor, T. 123, 128

electronic civil disobedience 69

Electronic Disturbance Theater 66, 69

Elsewhere: Anderswo 252, 252

equipped time 118, 121-2

erasure 174

Ericson, R. 68

everyday life, gamification $157-8$

expectations, technologies of social

mediation 25,26

exploration 241

Facebook 109-10

facial recognition 231-2

familiarity, and place attachment 107

family, as site of nomos 22

Farman, J. 127, 137, 156

Farmville 143

Feijóo, C. 139

filter bubbles 45

Fischer-Lichte, E. 123

Flanagan, M. 135

flash mobs 125

Foldit 151-2

Forlano, L. 190, 194

Forum Artis: National Museum, Oslo 213; as situated simulation (sitsim) 213-16

Foursquare 9, 65, 153; badges 94-5; chance discoveries 45 ; changing use 37-8; check-in screens 87; check-ins 108-9; choice of 40; competition and gaming 36-8; context and overview 33-5; databases 43, 91; features 86-8; loyalty 85 ; mayorship change message 93; mayorships 92-4, 108-9; as outsourcing 42; "Radar" option 96; relevance 45; selection algorithms 43-4; signal/noise ratio 38-9; study methodology 35 ; summary and conclusions 44; targeting and captivating users $36-9$; tech-savvy users 39,44 ; use of rewards $36-8$; venue creation 91-2; see also place attachment

"freemium" model 143

Friedberg, A. 133

friendships, maintaining 20
Frith, J. 85, 108, 126

Fullilove, M.T. 107

Galloway, A. 68

gamification 10,37; advertising revenue 151; applications 151-2; Belly 157; context and overview 149-50; discussion 159-60; domestic space $157-8$; history 151 ; hybrid spaces 150 , 155-9; issues raised 159-60; and mobile technologies $153-5$; and mobility 152-3; Monopoly 149, 150, 152, 156; playfulness 155-9; process 150-1; Shopkick 149, 150, 154-5, 156; summary and conclusions 160-1; technique 155; use of 154

Gamification by Design (Zicherman and Cunningham) 153

Gane, N. 156

gaze switching $50-1,54-8, \mathbf{5 5}, \mathbf{5 6}, \mathbf{5 7}$

Gemeinschaft 7, 19; continued role of mobile communication 29-30; digital 21-2; meaning 20; tension with Gesellschaft 22-4, 29

Geographic Information Systems (GIS) 65, 169

GeoGuessr 144-5

geosocial networks 42-4

Gesellschaft 7, 19; continued role of mobile communication 29-30; meaning 20

Giddens, A. 21

Gifford, R. 107, 108

gift time $117-18$

GIS 230-1

Global Positioning System (GPS) 4, 8, 65

globalization: vs. borderization $73-8$; as fallacy 78

Goffman, E. 119, 120, 121, 122

Goodwin, C. 56

Google Maps 107

Gordon, E. 72, 127, 173, 181, 183, 189

Goulet, R.-M. E. 125

GPS ankle bracelets $65-6$

GPS technologies 230-1

Green Button 150

Grosz, E. 243, 248

group utility 24-6

Guattari, F. 247, 248

Habermas, J. 19

haecceity 247

Haggerty, K. 68 
Hampton, K. 21, 22, 48, 189

Harvey, D. 160

heat-sensitive (thermo) cameras 231-3

Heidegger, M. 127

Hidden London 107-8

Hjorth, L. 135

homes, gamification 157-8

human techsourcing 151

hybrid cities, walking in 41

hybrid spaces 6,79 ; city transit $126-8$;

gamification 150; and interfaces 157;

nature of 242

hyper attention 62

ICT toolbox 12; camera tracking 231-2; Centre for Mobilities and Urban Studies (C-MUS) 226-7; contents 233-4; context and overview 224-5; Kennedy Project 232-3; manual 233-4; Mobility and Tracking Technology (MoTT) 227-8; need for 228-9; networked technologies 225-6; social knowledge 233; summary and conclusions 234-5; technologies 230-3; see also urban design

identity, and place attachment 108-9

ideologies, shared 22

immigration policies, United States 66-7

In the Metro (Augé) 129

inclusion 230

indirect augmented reality 209

individual addressability 20

industrialization, and control 20

information: retrieval and sharing 112; withholding 153

information and communication technologies (ICTs): development of 188; and location 189; place-making 189; suggested effects 188

information control technologies 20

information networks 103

infrastructure 191

interaction design 191

interactions: changing modes of 88 ; microcoordination of 34 ; ritual 22

interdisciplinary mobilities research 2-3 interfaces 156-7; and hybrid spaces 157 Ishii, K. 23

isolation, on public transit $120,125,128$

Ito, M. 23

Jackson, J.B. 246

Jacobs, J. 170-1, 184
Jain, J. 118, 121

Jensen, O.B. 117, 122, 129, 189, 202

Johnson, S. 1

Jørgensen, K.M. 229

just in time production $20-1$

kairos 35, 44

Kalnins, K. 241

Katz, J. 25

Kaufman, S. 1

Kellner, H. 21-2

Kennedy Project 232-3

kinesthetic engagement 241

Kishino, F. 209

Kitchin, R. 224, 225, 226-8, 234

Kleihues + Schuwerk 213

Klett, M. 210

Kool, L. 35

labeling, location-aware technologies 66 landscape approach 243-7

landscape, concept of 244-7

Landschaft 245-6

Lee, D.H. 28

Lefebvre, H. 7, 33, 34-5, 39-40, 123

legitimations 26

leisure 152-3

Lenhart, A. 23

Lenz, B. 190

Licoppe, C. 23

Life is Magic 141-5

liminal space 119-23, 128

Ling, R. 22, 23, 24, 33, 34, 120

location: commodification 10; conception of 102; as distinct from place 103; as mediator 4-5; and sense of place 9 , $103-4,112$

location-aware technologies: labeling 66; privacy and control 65-6

location-based mobile games (LBMGs) 10; Camover 2013 145; commercial application 149,150,152, 154; context and overview 132-3; distribution platforms 139-40, 143; "freemium" model 143; funding and profit 137-8; GeoGuessr 144-5; as killer apps 139-41; labor of play 141-5; Life is Magic 141-5; market 140-1; origins 133-6; and rise of app economy 136-9; summary and conclusions 145-6; use of 33; see also Foursquare

location-based services 4 
location-based social networks 4,7

locational information, use of 102

locations, regulation of 79

locative art, aims 134

locative art projects 134

locative media art 12-13

locative media artists, scope 3-4

locative media, landscape approach 243-7

locative media, use of term 241-2

locative mobile social networks (LMSNs), use of 33

London: overview 104-5; see also sense of place

Los Angeles, city transit 128-9

Low, S.M. 85, 89, 95

loyalty programs 151

Lozano-Hammer, R. 134

Luckmann, T. 26

Lynch, K. 170-1, 184, 211

Lyons, G. 118, 121

Mainsah, H. 177

management of attention 48

Mann, S. 49

Manosevitch, E. 183

mapping: as interpretation 176; mobile 172-6; participative 173; postmodern 170-1; tools 173; see also cultural mapping

Marcus, C.C. 109

marketing 149, 151

Mashable 139

mass production 20-1

mayorships 92-4, 108-9

McCrea, C. 143

McDonalds see Monopoly

McLuhan, M. 207, 242

media, situated and sensory 207

Meethan, K. 111

memory, and place attachment 109-11

Merleau-Ponty, M. 248

Merrell Lynd, H. 28

messiness, urban environments 58

methodologies, developing 5

metis 41

Mexico-US migration 74

Meyrowitz, J. 102

micro-coordination, of interactions 34

migration 174; Mexico-US 74

Milgram, P. 209

Miller, G.B. 127

Milligan, M.J. 89

\section{minimalism 243}

Mitchell, W.J. 157

mobile annotations 127

mobile cities 10-11

mobile communication, continued role in

Gesellschaft and Gemeinschaft 29-30

mobile communication studies, scope 3

mobile experience, use of term 241-2

mobile mediality 72

mobile multi-activity 7-8; advantages of methodology 60; context and overview 48-9; equipment 51-3, 52, 53; gaze switching 50-1, 54-8, 55, 56, 57; management of attention 48; methodology 51-4; participants 54; research 49; research approach 49-51; summary and conclusions 61-3; temporal mismatches 58-61, 59, 60; textures 62

mobile phones: and cars 28-9; choice to own 19-20; forms of use 22-4; progress bars 55-6; range of uses 3

mobilities, scope 2

mobilities studies, approach 67

mobility: and internet use 188-9; of place 112

Mobility and Tracking Technology

(MoTT) 225, 227-8

Monopoly 149, 150, 152, 156

Montréal see city transit

Morrison, A, 177

motility 2

multi-activity, use of term 49

multi-tasking, forms of 49

music: buskers 125 ; on city transit 122

NasMus prototype 215-19

National Museum, Oslo 213-16

naturalization 157

navigation $105-8$

net localities 72

network capital 2, 68

network communities 21

network orchestration 42-4

networked city 167, 220

networked technologies: as part of city

229-30; and programmable city $225-6$

Ng-Chan, T. 127

niches 26

No Places With Names: A Critical Acoustic Archaeology 252-5, 253

Nobis, C. 190

nomos 21-2 
non-places 9,88

Non-Places (Augé) 128

nonreciprocal looking 122

Nora Project 233

Norway, mobile phone use 23

Norwegian Public Construction and Property

Management (Statsbygg) 213-14

nostalgia 110

occasions 40-1

oculometric video recording 49-50

Okabe, D. 23

online place attachment 95-7

OpenStreetMap 173

origination 174

Oslo see situated simulation (sitsim)

Oslo bombing, use of mobile phones 30

ownership 92-4, 95

\section{Pacmanhattan 136}

Pan-American Mobilities Network 2, 6 paradigm of identity 171

parametric design 211

Pariser, E. 45

performance art, border crossing as 72

performance space, city transit 123-4

Performance Theory (Schechner) 124

performative urban spaces 230, 232-3

pervasive games $149,154,155-6$

Pew Research Center 74

phenomenology 248

physical configuration, of place 11

Picon, A. 191, 202

place: detachment from 103; as distinct

from location 103; identity 104 ;

mobility of 112; physical configuration

of 11; properties of 190; sense of see sense of place;

place attachment 9; collective 96; context and overview 85-8; discussion 95-7; events 95; and familiarity 107; and identity 108-9; and memory 109-11; methodology 89-90; mobile technology and place 88-9; online 95-7; ownership 92-4, 95; place naming 91-2, 95; results 90-5; sense of place 103-4; shared meanings 88-9; summary and conclusions 97 ; temporality 96 ; as theoretical concept 88-9; see also Foursquare

place attachment theory 85-6, 95; application 89 place-making: border crossing as 72 ;

Transborder Immigrant Tool 72-3

place naming 91, 95

playfulness 149,154 ; and chance 34 ; and

hybrid spaces $155-9$

playification 159

pleasure, of urban travel 117-18, 128

Plug-in-City 201-2

Point de Fuite (Goulet and Dumas) 125

political resistance 8

politics of mobility $66,73-4,79$

portable devices, video recording 48-50

Poster, M. 156

power-geometries 160

pragmatic texture 62

privacy, location-aware technologies 65

private geographies 91

Proboscis 172, 173

programmable city: and networked

technologies 225-6; see also ICT toolbox

programmable urbanism 12

progress bars 55-6

Project 55 (COHDS) 127-8

prospective indexicals 56

protest 167

Protevi, J. 247

protocols 68

public participation, enactment 88

public spaces, personal uses 88

Putnam, R. 7

Québec City 189; WiFi hotspots and users 192-3; WiFi users, demographics

194-5; see also urban design

$R 2140$

radio frequency identification (RFID) 231

reciprocal expectations, technologies of social mediation 25,26

red lights, traffic management 54-5

Red Pavilion 233

Red Robot 140, 141

regulation, locations, spaces and territories 79

relational programming 200-3

Relph, E. 104

research, focus of 101

retrieval 112

RFID 231

Rheingold, H. 48

rhythm, city transit 123

right points in time 35 
Riley, R. 109

ritual interactions 22

ritual, use of term 120

Robbins, E. 211

robot Santa Claus 233

Roman Forum 209-11, 210

routing, of borders 67-9

Rueb, T. 12-13, 134

ruggedness 62

rush hour 123-4

Santa Claus robot 233

Scannell, L. 107, 108

Schechner, R. 124

screen-capture recording 50

sedentarist theories 67

Seek 'n Spell 138

sense of place 9; context and overview

101-2; discussion 112-13; findings

105-11; and location 9; location 103-4,

112; methodology 104-5; mobility 104; navigation 105-8; place attachment

103-4; research literature 102-4; sharing

103; summary and conclusions 113

sequential texture 62

Serendipitor 107

serendipity 45

Serra, R. 244

Shane, G. 176

sharing 112

Sheller, M. 6, 67, 68, 72, 188

Shepard, M. 172, 183

Shklovski, I. 10

Shopkick 149, 150, 154, 156

shopping centers 27

Simmel, G. 119, 122, 224

Simonsen, K. 102

situated simulation (sitsim) 11-12;

augmented realities 208-13; context and overview 207; developmental approach

208; examples 214, 216-18; Forum

Artis 213-16; future research 219-21; of future topics 211-13; NasMus prototype 215-19; National Museum, Oslo

213-16; of past topics 209-11; Roman

Forum 210; summary and conclusions

219-21; trial and evaluation 216-19; see also urban design

smart meters $157-8$

smart mobs 125

Smithson, R. 243-4

smooth space 247
SMS 230-1

social cohesion 7; communal vs. associative society $20-1$; context and overview 19-20; digital gemeinschaft 21-2; summary and conclusions 29-30; technologies of social mediation 24-6; tension between Gemeinschaft and Gesellschaft 22-4, 29; transport, coordination and communication 27-9 social detection $231-2$

social facts $24-5$

social life, coordination of 34-5

Social Signal Processing (SSP) 232

social ties $21-2$

socialization, use of cars $27-8$

society, communal vs. associative $20-1$

Söderblom, K. 120

software orchestration 43

sound walks 127

sovereignty 75

spaces: regulation of 79; use of 189

spatial mapping 196-200

Stalbaum, B. 69

Statsbygg 213-14

Steen, G. 139

"Stéréobus" (Audiotopie) 126-7

Stoughton Lynd, R. 28

Streetscape 11, 168-9; aims 176; background 172; basis 177 ; as collaborative project 180-1; discussion 183-4; displaying results 177; findings 181, 183; information retrieval 181; interactions 178, 184; international application 183; outcomes 180-3, 182; sample point notation 179; summary and conclusions 184-5; testing 178-80; using 177; see also Urban Gallery; YOUrban project

striated space 247,248

subjectivity 242

subversion 8

surveillance $10,153-4,156$; camera tracking 231-2

surveillant assemblages 68

Sutko, D. 33, 34, 154

swarm badge $94-5$

symbiosis, cars and mobile phones $28-9$

Taiwan Strait 174

technologies of social mediation 24-6; cars and mobile phones $28-9$

technology, fear of 229 
teleological navigation 34

temporal mismatches, mobile multiactivity $58-61, \mathbf{5 9}, \mathbf{6 0}$

temporality, and place attachment 96 territoriality 67

territories: regulation of 79 ; as social creations 67 ; and sovereignty 75

The Beast 135

The Economist 151

The Metropolis and Mental Life (Simmel) 122

"The phenomenon of mobility at the Frankfurt international airport" (Söderblom) 120-1

The Practice of Everyday Life (de Certeau) 40-1

The Presentation of Self in Everyday Life (Goffman) 121

theory of moments $34-5,39-40$

theory of occasions $34-5,39$

"There to Hear: Placing Mobile Music" (Thulin) 126-7

Thrift, N. 220

Thulin, S. 126-7

time out 121

togetherness 7,111

Tönnies, F. 7, $19 \mathrm{ff}$

Toronto, city transit 129

Townsend, A. 200

"Trace" (Rueb) 12-13

traffic management 54-5

Transborder Immigrant Tool 8; aims 71-2; as art and assistance 73; availability 75; context and overview 65-7; description 69-71; development of 69; globalization vs, borderization 73-8; implementation 71; interface 70; investigations into 76; media representation 75-6; Mexico-US migration 74; place-making 72-3; poetic sustenance 72 ; research questions 66 , 73-8; responses to 75-6; routing of borders $67-9$; summary and conclusions 78-9; terrorism 75

transdisciplinarity $1-2,5$

transduction 225

transformation 174

transition time 121

translation 225

transport, coordination and communication $27-9$

transportation see city transit

travel time 121-2, 128
Turkle, S. 7

Turner, V. 119, 120, 124, 125

Tuters, M. 134

United States, immigration policies 66-7

University of Washington 151-2

urban augmented reality (UAR) 212-13

urban design: background to research

189-90; changing perspectives 202;

characteristics of WiFi user groups 193;

context and overview 188-9;

distribution of WiFi hotspots 196; geographic disparities 195-7; hotspots visited 198; methodology 192; most frequented hotspots 197-8, 197, 198;

Pub Galway 199; relational

programming 200-3; spatial mapping

196-200; summary and conclusions

203; types of WiFi hotspots 196; WiFi

hotspots and users 192-3; WiFi users, demographics 194-5; see also situated simulation (sitsim)

urban environments, messiness 58

Urban Gallery 169, 173-6; see also Streetscape

urban mapping 169-72

urban mark-ups 127

urban planning: alternative approaches

171; compartmentalization 228-9;

networked technologies 229-30; see also ICT toolbox

Urban Tapestries 172-3

urbanization 34

Urry, J. 67, 152, 188

Van Gennep, A. 120

Varnelis, K. 133, 146

venue creation 91-2, 95

video recording, portable devices $48-50$

Virilio, P. 159

"Virtual Hiker" algorithm 69, 71

visibility paradox 38

Vukov, T 68

Walking City 201-2

walking in the city $40-1$

Watts, L. 122

wearable computing 49

Weber, M. 25

Wellman, B. 21, 22

Where Good Ideas Come From (Johnson) 1

WiFi hotspots 11; distribution 196; 
geographic disparities of WiFi hotspots 195-7; hotspots visited 198; location and use 190-2; most frequented 197-8, 197, 198; Pub Galway 199;

representations of users 200; spatial mapping 196-200; types 196; see also urban design

WikiMe 107

will to connection 229, 235
Wilson, M.W. 153

Wither, J. 207, 209

YOUrban project 168, 169; see also Streetscape

ZAP Québec see Québec City Zicherman, G. 153

Zynga 141 


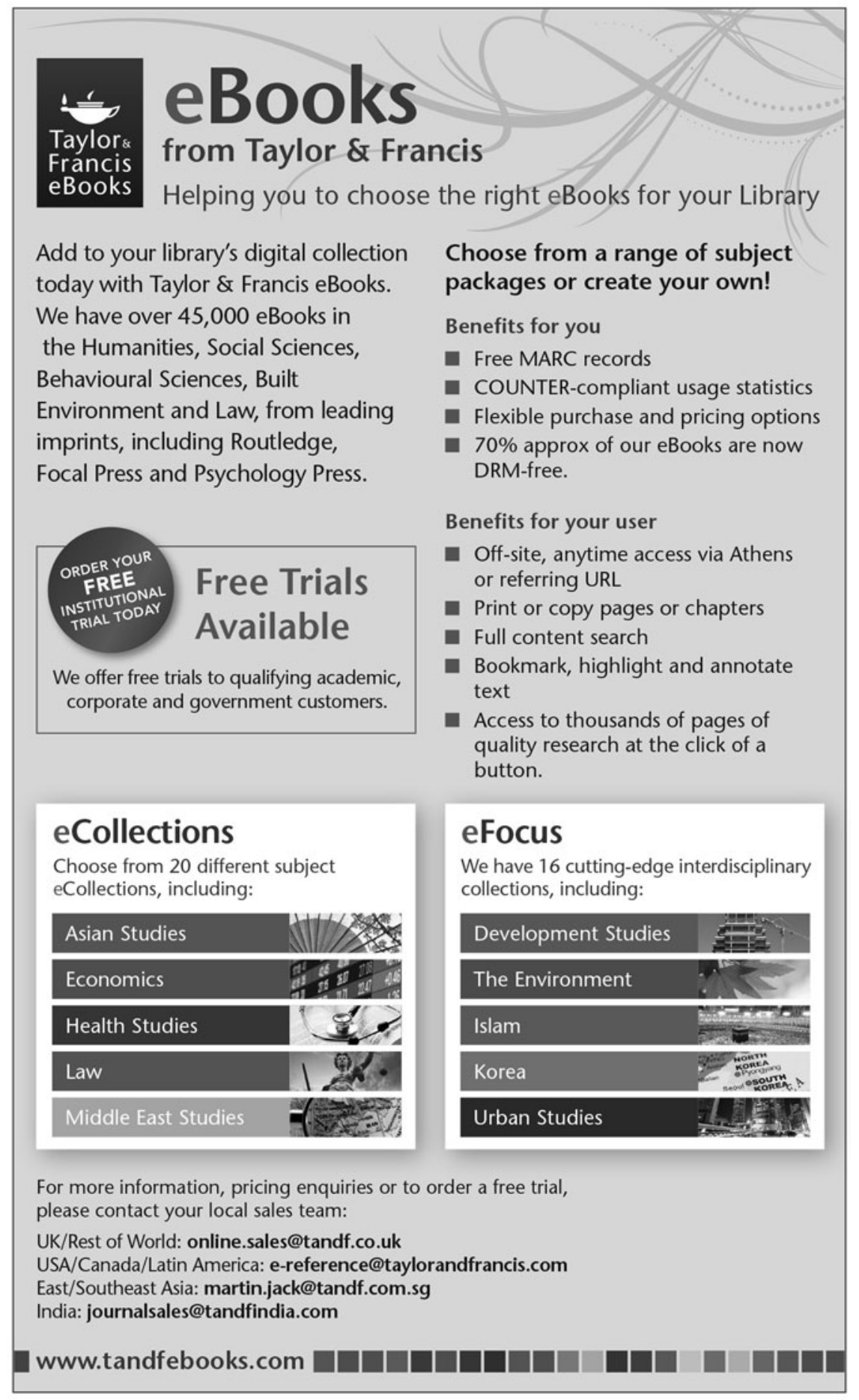

